\author{
Masterarbeit \\ im Studiengang „Angewandte Medien- und Kulturwissenschaften“ \\ Fachbereich Soziale Arbeit.Medien.Kultur \\ Hochschule Merseburg
}

\title{
Schule als Schutzraum vor sexualisierter Gewalt und Ort Sexueller Bildung aus Lehrer*innenperspektive
}

angefertigt von: Maria Urban

Matrikel: $\quad 15238$

angefertigt bei: $\quad$ Prof. Dr. Heinz-Jürgen Voß

Datum der Abgabe: 26.10.2018 


\section{Inhaltsverzeichnis}

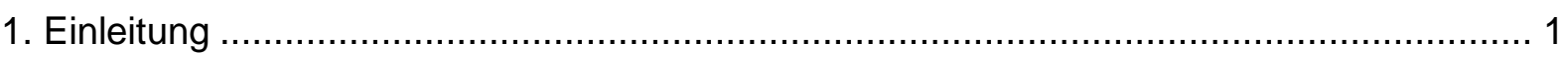

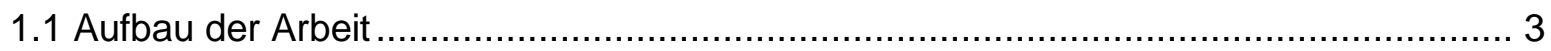

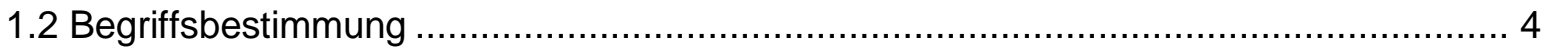

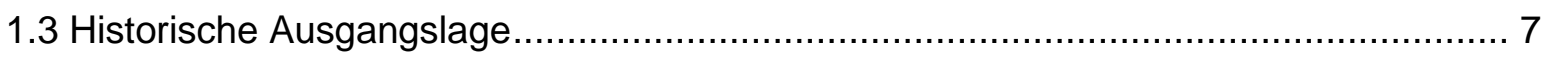

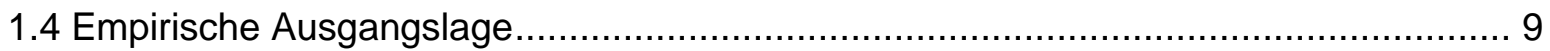

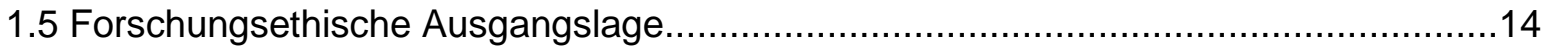

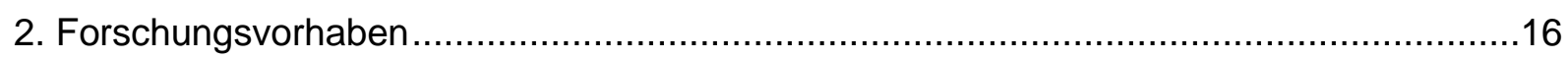

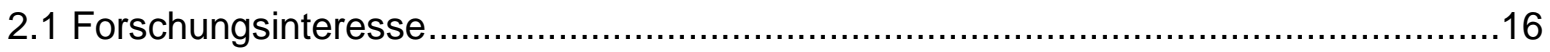

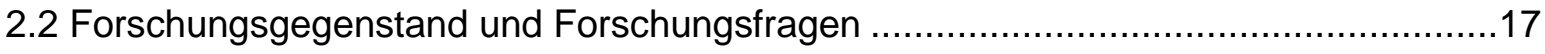

3. Sexuelle Bildung in Schulen - Theoretischer Kenntnisstand ...................................18

3.1 Historische Ausgangslage Sexueller Bildung an Schulen........................................18

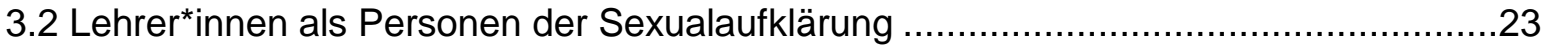

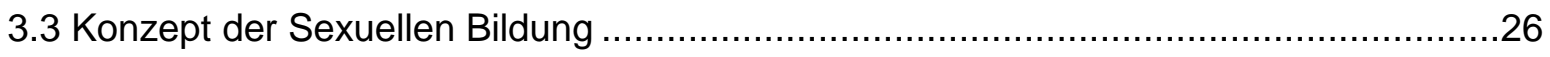

3.4 Qualifizierungsstand von Lehrkräften mit Fokus auf Studium und Fortbildung ...........29

3.5 Aktuelle Entwicklungen in der Forschungslandschaft.......................................32

4. Schule und sexualisierte Gewalt - Theoretischer Kenntnisstand ..................................34

4.1 Sexualisierte Gewalt in der Lebenswelt von Kindern und Jugendlichen .....................34

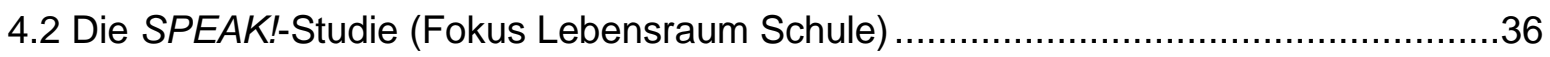

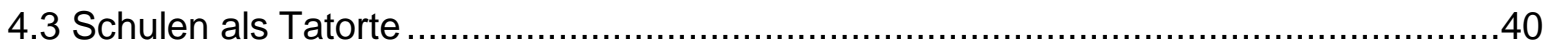

4.3.1 Sexualisierte Gewalt im Lebensraum Schule...............................................41

4.3.2 Risikofaktoren für sexualisierte Übergriffe in schulischen Institutionen .................44

4.4 Förderschulen als besonders gefährdende Institution .........................................45

4.5 Prävention sexualisierter Gewalt im Schulalltag .................................................48

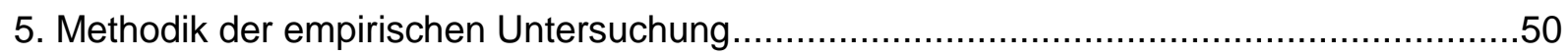

5.1 Begründung der qualitativen methodischen Vorgehensweise ...............................50

5.2 Die Auswahl der Stichprobe und ihre Besonderheiten ........................................50

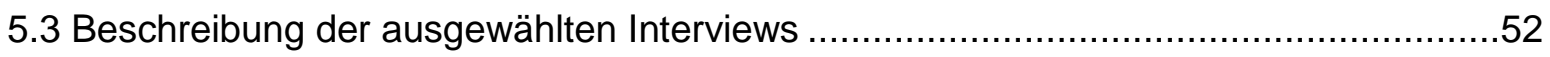

5.4 Forschungsethische Reflexion zum Umgang mit den Interviews .............................53 


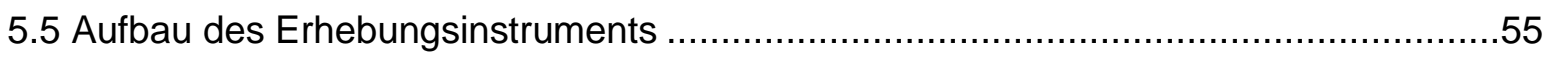

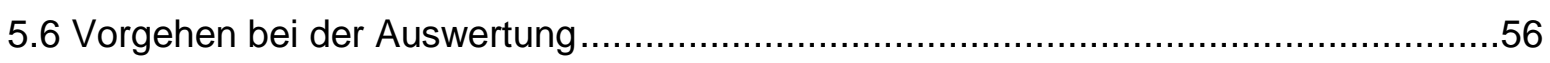

5.6.1 Anwendung der qualitativen Inhaltsanalyse nach Mayring ................................56

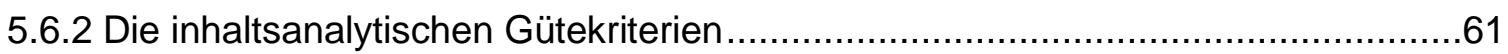

5.6.3 Die Entwicklung des Kategoriensystems als Grundlage der Analyse...................61

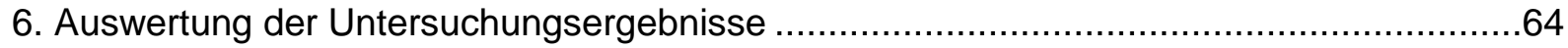

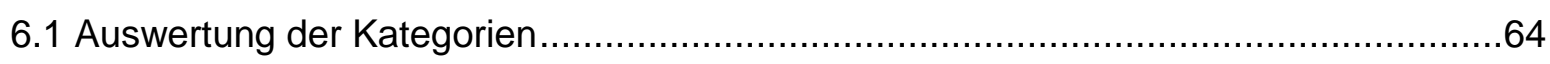

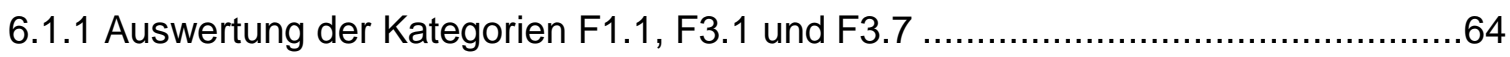

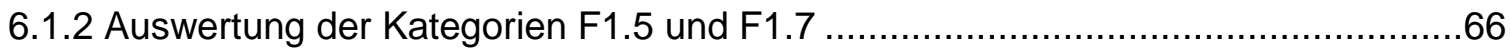

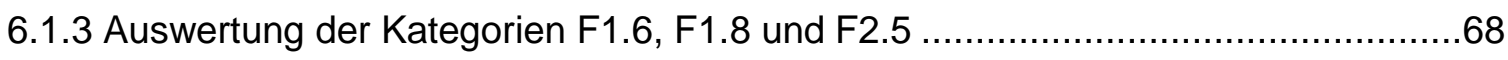

6.1.4 Auswertung der Kategorie F3.4 …........................................................... 72

6.1.5 Auswertung der Kategorien F1.2, F1.3 und F1.4 _........................................74

6.1.6 Auswertung der Kategorien F2.1, F2.4, F2.7 und F2.8 ................................77

6.1.7 Auswertung der Kategorien F2.2, F2.3 und F2.6 .......................................79

6.1.8 Auswertung der Kategorien F4.1, F4.2, F4.3 und F4.4 _............................... 80

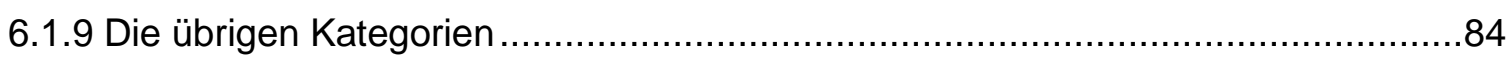

6.2 Kernaussagen und zentrale Thesen der Auswertung ............................................84

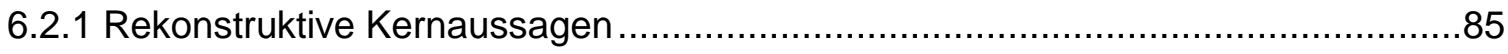

6.2.2 Zentrale Thesen auf Grundlage der Auswertung ........................................... 86

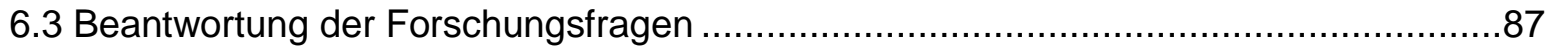

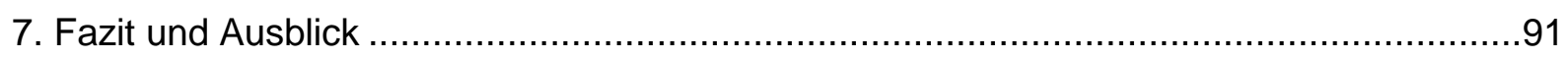

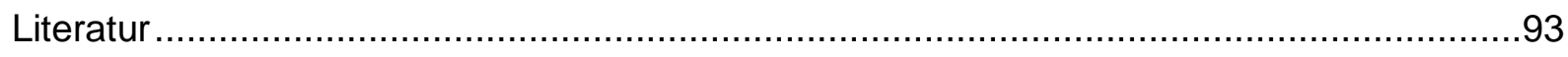

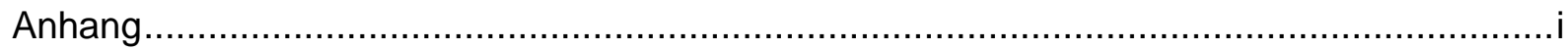

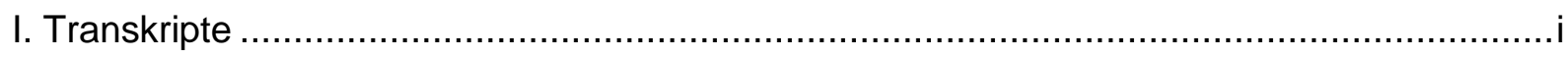

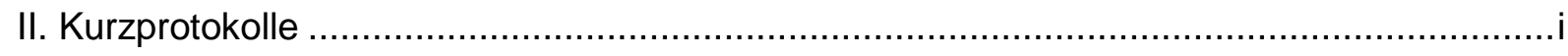

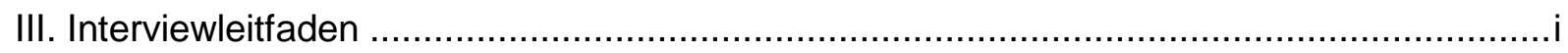

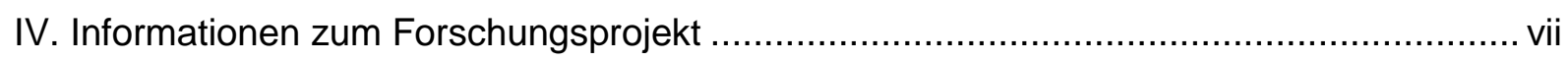

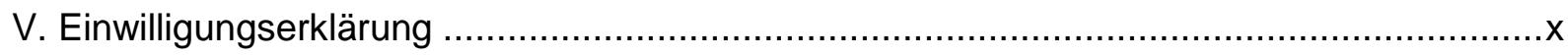

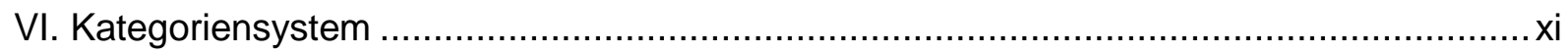


Abbildungsverzeichnis

Abb. 1: Erzieherische Hilfen und sonstige Leistungen, Teil 1......................................... 10

Abb. 2: Erzieherische Hilfen und sonstige Leistungen, Teil 2 ....................................... 10

Abb. 3: Übersicht Datenerhebung......................................................................... 11

Abb. 4: Personen der Sexualaufklärung. Jugendliche nach Geschlecht ............................23

Abb. 5: Personen der Sexualaufklärung. Jugendliche nach Geschlecht ............................23

Abb. 6: Personen der Sexualaufklärung. Jugendliche nach Herkunft.................................24

Abb. 7: Sexualaufklärung in der Schule. Langzeittrend deutsche Jugendliche nach Region.24

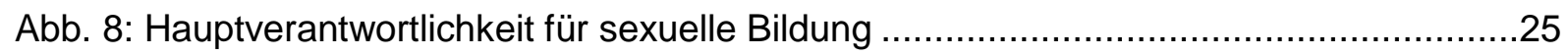

Abb. 9: Verteilung Ausbildung in Sexualerziehung in Prozent..........................................30

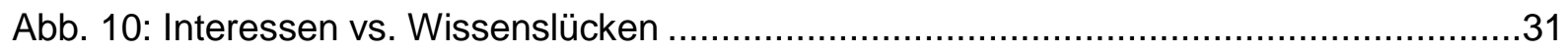

Abb. 11: Beziehung zum Täter bei Opfern in verschiedenen Altersgruppen .........................35

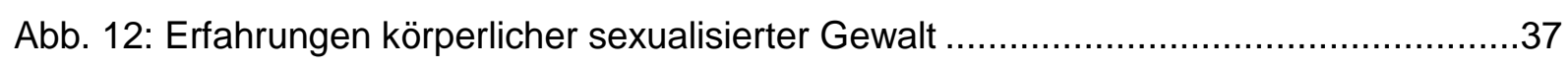

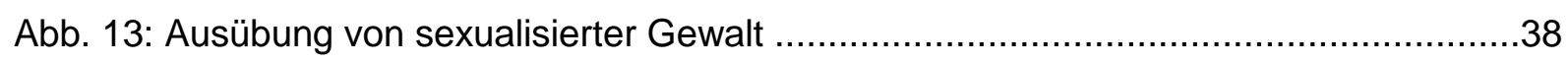

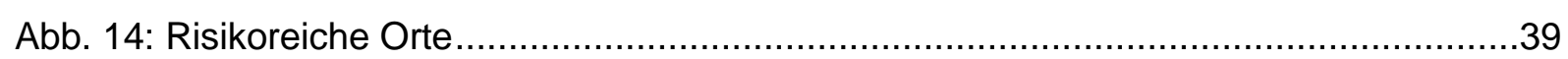

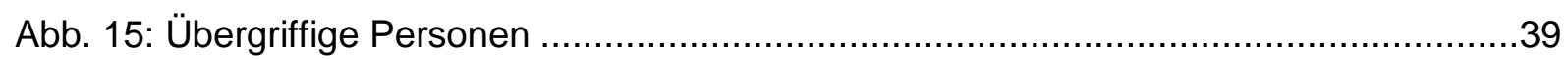

Abb. 16: Prävalenz körperliche sexualisierte Gewalt (Förderschüler*innen) ........................47

Abb. 17: Prävalenz Ausübung sexualisierter Gewalt (Förderschüler*innen) …....................47

Abb. 18: Allgemeines inhaltsanalytisches Ablaufmodell..............................................58

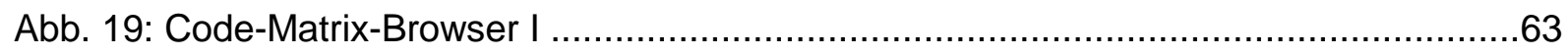

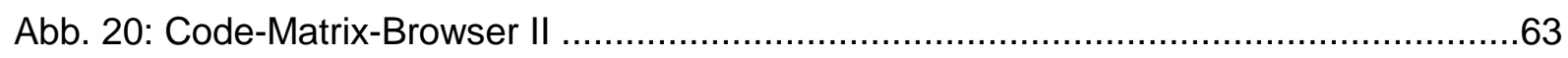




\section{Einleitung}

Der Unabhängige Beauftragte für Fragen des sexuellen Kindesmissbrauchs (UBSKM), Johannes-Wilhelm Rörig, spricht in einem 2017 veröffentlichten Dokument von mehr als 12.000 strafrechtlich relevanten Fällen von sexuellem Kindesmissbrauch ${ }^{1}$ im Jahr 2016. Neben den ca. 7.000 polizeibekannten kinder- und jugendpornografischen Vorfällen sowie Übergriffen auf Jugendliche kommen alle Geschehnisse des sogenannten Dunkelfelds hinzu, also jene, die der Polizei nicht bekannt werden. Schätzungen legen die Annahme nahe, dass in jeder Schulklasse ein bis zwei Schüler*innen von sexualisierter Gewalt betroffen sind oder waren (vgl. UBSKM 2017: 1f).

Der Schutz von Kindern und Jugendlichen und die Prävention sexualisierter Gewalt hat in den vergangenen Jahren einen neuen, bedeutenden gesellschaftlichen Stellenwert erreicht. Dem voraus gingen diverse Skandale und großflächige Aufdeckungen von Übergriffen auf Kinder und Jugendliche insbesondere in schulischen Institutionen und daraus resultierende Initiativen des Bundes, Theorie und Praxis zum Schutz der Heranwachsenden zu stärken und zu professionalisieren (vgl. Bundesministerium für Bildung und Forschung o.J).

Die hohen Fallzahlen zeigen auf, dass Kinder und Jugendliche gefährdet sind, von sexualisierter Gewalt betroffen zu sein und deshalb geschützt werden müssen. Ansatz dafür kann und sollte eine Erziehungshaltung sein, die ganzheitlich von einem präventiven Gedanken geprägt ist und nicht nur auf Präventionsangebote für die Kinder und Jugendlichen fokussiert. Viel mehr setzt Prävention zum einen damit an, dass alle Personen, die zur Lebenswelt von Heranwachsenden gehören, aktiv werden müssen, um eine Kultur der Grenzachtung zu entwickeln und zu vermitteln (vgl. Vogelsang/Riesau 2018). Zum anderen ist Prävention untrennbar verbunden mit einer generellen Auseinandersetzung mit Sexualität und der eigenen Geschlechterrolle, sexuellen Orientierung und sexuellen Selbstbestimmung (vgl. Riesau 2018). Auch Schulen müssen und sollen zum Schutz von Kindern und Jugendlichen und der Prävention sexualisierter Gewalt beitragen. Für den UBSKM stehen Schulen sogar in einer so hohen Verantwortung, dass er festhält, dass es „nur durch das Engagement jeder Schule (...) schrittweise zu einem Rückgang der unverändert hohen Fallzahlen kommen [kann]“ (Rörig o. J.).

In den vergangenen Jahren hat die Thematik Schule und Sexualität nicht zuletzt aufgrund der

\footnotetext{
${ }^{1}$ Der UBSKM definiert sexuellen Kindesmissbrauchs als jene sexuellen Handlungen, „die an oder vor Mädchen und Jungen gegen deren Willen vorgenommen wird oder der sie aufgrund körperlicher, seelischer, geistiger oder sprachlicher Unterlegenheit nicht wissentlich zustimmen können" (Arbeitsstab des UBSKM (3) o.J.). Ein zentrales Merkmal der übergriffigen Situation ist das Ausnutzen der Machtund/oder Autoritätsposition des*der Täters*in zur Bedürfnisbefriedigung gegenüber dem jungen Menschen.
} 
zahlreichen Aufdeckungsvorgänge an Bedeutung gewonnen (vgl. Schmidt 2014; 249). Neben der grundlegenden Einigkeit darin, dass Schulen aktiv zum Kinderschutz beitragen sollen, gibt es sowohl gesellschaftlich als auch wissenschaftlich bisher nur punktuelle Auseinandersetzungen damit, welchen Beitrag sie tatsächlich leisten (können). Bisher ebenfalls vernachlässigt ist eine Betrachtung des Verhältnisses von Schule und Sexueller Bildung, welche aus meiner Sicht ein ganzheitliches Herangehen an das Phänomen Sexualität und die Auseinandersetzung mit all seinen Facetten ermöglicht. Sexuelle Bildung ist untrennbar mit der Reflexion und Überwindung heteronormativ besetzter Grundannahmen und einer Auflösung von streng polaren und mitunter diskriminierenden Haltungen verbunden. Sie überwindet dabei nicht nur selbst diese gesellschaftlichen Hürden, sondern bereitet durch Benennung tabuisierter Themen, dem Verwenden altersgerechter Methoden und einer Interaktion zwischen verschiedenen Generationen den Weg, dass Sexualität offen im gesellschaftlichen Diskurs, vor allem aber in der Lebenswelt von Kindern und Jugendlichen verhandelt werden kann. Sie ist in Kombination mit der Akzeptanz, Befürwortung, Stärkung von und Befähigung zu sexueller Selbstbestimmung die Grundvoraussetzung für eine Akzeptanz von Diversität, dem kritischen Hinterfragen vermeintlich geltender Normen und der Auseinandersetzung mit vielfältigen Lebensmodellen und Beziehungsformen. Gleichzeitig eröffnet sie beispielsweise Lehrkräften und Eltern die Möglichkeit eines ressourcenorientierten Herangehens ohne allein nur die Gefahren von in jungen Jahren gelebter Sexualität abwenden zu wollen. Sexuelle Bildung vereint damit sehr viele Aspekte, die insbesondere auf eine positive Auseinandersetzung mit der eigenen Sexualität und eine Enttabuisierung des Themas hinwirken. Nur wenn es gelingt, das Thema Sexualität als solches zu enttabuisieren, wird ein Sprechen über sexualisierte Gewalt ermöglicht. Damit ist Sexuelle Bildung gleichzeitig ein zentraler Baustein in der Prävention sexualisierter Gewalt. Von diesem Standpunkt aus ist die Hypothese naheliegend, dass Sexuelle Bildung und die Befähigung zur sexuellen Selbstbestimmung maßgeblich darüber entscheiden, wie erfolgreich die Prävention sexualisierter Gewalt gelingen kann. Sexualisierte Gewalt selbst kann nicht losgelöst von gesellschaftlichen Herrschafts- und Machtverhältnissen getrennt betrachtet werden, da erst sie sexualisierte Übergriffe überhaupt ermöglichen und begünstigen (vgl. Wiesental 2017: 8). Umso wichtiger ist die ganzheitliche Einbeziehung Sexueller Bildung in den schulischen Alltag, da sie allen Generationen Anlass gibt, die bestehenden Strukturen zu hinterfragen, gewaltbegünstigende Aspekte zu identifizieren und aufzubrechen. Weiterhin kann Sexuelle Bildung insofern präventiv wirken, als dass sie Kinder und Jugendliche darin stärkt, mögliche Risiken zu erkennen und sich gegen sexualisierte Grenzverletzungen erfolgreich(er) zu wehren.

Die vorliegende Arbeit untersucht deshalb, inwieweit die in Schulen tätigen Lehrkräfte tatsächlich einen Beitrag dazu leisten können, die Prävention sexualisierter Gewalt 
voranzubringen und für wie kompetent sie sich selbst einschätzen. Für ein umfassendes Verständnis wird dafür untersucht, inwieweit Studieninhalte des Lehramtsstudiums Lehrer*innen befähigen, professionell zu handeln und Sexuelle Bildung zum Bestandteil schulischen Alltags zu machen.

\subsection{Aufbau der Arbeit}

Der Aufbau der Arbeit ist gegliedert in eine logische Hinführung zur eigenen empirischen Erhebung dieser Masterthesis.

Nach einer einführenden Begriffsbestimmung und der Klärung des zugrundliegenden Verständnisses der verwendeten Begrifflichkeiten folgt die Darstellung der historischen und empirischen Ausgangslage der Untersuchung. Dazu zählt ein Abriss der Entwicklungen seit der Aufdeckungswelle von Missbrauchsfällen aus dem Jahr 2010 sowie die Erläuterung erster Ergebnisse des Forschungsprojektes „Schutz von Kindern und Jugendlichen vor sexueller Traumatisierung" an der Hochschule Merseburg. Da der qualitative Forschungsanteil dieser Masterarbeit maßgeblich an die Inhalte des Merseburger Projekts anschließt beziehungsweise einen Teil dessen darstellt, wird auch das dortige Untersuchungsvorgehen aufgezeigt. Die Einleitung wird abgerundet von einem Einblick in die forschungsethische Ausgangslage, welche im späteren Verlauf der Arbeit Grundlage der forschungsethischen Reflexion zum eigenen Vorgehen ist.

Das zweite Kapitel der Arbeit fokussiert das eigentliche Forschungsvorhaben. Zunächst wird dabei das Forschungsinteresse konkretisiert. Es folgt die Skizzierung des Forschungsgegenstandes und die Formulierung von vier Forschungsfragen, mit deren Hilfe sich im weiteren Verlauf dem Forschungsinteresse genähert werden soll.

Im dritten Kapitel findet sich eine intensive Betrachtung des theoretischen Kenntnisstands zur Sexuelle Bildung. Zunächst werden Aspekte der historischen Entwicklung Sexueller Bildung an Schulen aufgegriffen und die Geschichte der schulischen Sexualaufklärung dargelegt. Nachdem Lehrer*innen als Personen der Sexualaufklärung identifiziert wurden, schließt sich die Erläuterung des Konzepts der Sexuellen Bildung an. Weiterhin wird der Qualifizierungsstand von Lehrkräften anhand empirischer Studien hinterfragt und ein kurzer Exkurs in aktuelle Entwicklungen der Forschungslandschaft unternommen.

Nachdem mit diesen Ausführungen ein umfangreicher Überblick zur Sexuelle Bildung gegeben wurde, bedarf es einer ebenso gründlichen theoretischen Auseinandersetzung mit dem Phänomen der sexualisierten Gewalt in schulischen Kontexten. Ausgangspunkt dazu stellt die Untersuchung sexualisierter Gewalt in der Lebenswelt von Kindern und Jugendlichen dar, welche um zentrale Ergebnisse der „SPEAK!-Studie“ ergänzt wird. Im Folgenden werden Schulen als Tatorte identifiziert und wird der Frage nachgegangen, in welcher Form sexualisierte Gewalt im Lebensraum Schule auftritt. Weiterhin werden wesentliche 
Risikofaktoren für sexualisierte Übergriffe in Schulen herausgearbeitet. Ein besonderes Augenmerk gilt anschließend den Förderschulen, welche aufgrund ihrer Konstitution als besonders gefährdende Institutionen anzusehen sind. Das vierte Kapitel schließt mit einem Einblick in die Umsetzung präventiver Angebote im schulischen Alltag.

Nach der theoretischen Rahmung stellt das Kapitel fünf den Übergang zur empirischen Erhebung dieser Masterarbeit dar. Die Begründung für das qualitative methodische Vorgehen sowie die Erläuterung der Stichprobenauswahl, ihrer Besonderheiten und die Beschreibung der zur Analyse herangezogenen Interviews gibt einen umfassenden Überblick über die methodologischen und inhaltlichen Voraussetzungen der Forschung. Es folgt eine intensive forschungsethische Reflexion zum Umgang mit den Interviews, welche auf den forschungsethischen Ausführungen im ersten Kapitel aufbauen. Anhand des Aufbaus des Erhebungsinstruments wird der den Interviews zugrundeliegende halboffene Leitfaden beschrieben. Eine detaillierte Erläuterung des Vorgehens bei der Auswertung beinhaltet zum einen die Darstellung der angewandten Methode - die qualitative Inhaltsanalyse nach Mayring - sowie die Auseinandersetzung mit den inhaltsanalytischen Gütekriterien. Mit der Entwicklung des Kategoriensystems wird, das fünfte Kapitel abschließend, die Grundlage der Auswertung aufgezeigt.

Im Rahmen der Auswertung, welche das gesamte sechste Kapitel füllt, erfolgt zunächst die Betrachtung der einzelnen im Kategoriensystem festgehaltenen Kategorien, welchen mithilfe der Software MaxQDA zahlreiche Textstellen zugeordnet wurden. Im weiteren Verlauf werden daraufhin Ableitungen getroffen, welche anschließend zur Beantwortung der Forschungsfragen herangezogen werden.

Die Masterarbeit schließt mit einem umfassenden Fazit und einem Ausblick auf zukünftige Forschungs- und Entwicklungsschwerpunkte.

\subsection{Begriffsbestimmung}

Im sexualwissenschaftlichen Feld gibt es zum Teil ein diverses Verständnis von gleichen oder ähnlichen Begriffen. Zunächst ist es deshalb notwendig, an dieser Stelle die in der Arbeit verwendeten Begriffe im zugrundeliegenden Verständnis zu erläutern.

\section{Sexualität}

Die Bundeszentrale für gesundheitliche Aufklärung (BZgA) spricht im Hinblick auf Sexualität von einem „existentielle[n] Grundbedürfnis des Menschen und ein[em] zentrale[n] Bestandteil seiner Identität und Persönlichkeitsentwicklung“ (BZgA (2) o.J.) Dieser Auffassung folgend wird Sexualität in der vorliegenden Arbeit als für jedes Lebensalter und jede Entwicklungsstufe eines Menschen relevant verstanden. Erwartungsgemäß äußert sich diese Relevanz bei 
jedem Individuum und in jeder Phase der Entwicklung verschieden. Sexualität, das Ausleben derselben und die Auseinandersetzung mit ihr steht in engem Zusammenhang mit Vorstellungen von Werten und Normen und damit der die*den Einzelne*n umgebenden Gesellschaft (vgl. ebd.). Sexualität ist sodann von physischen, psychischen und sozialen Einflüssen gekennzeichnet und nicht beschränkbar auf eine einseitige Betrachtung wie die Voraussetzung zur Fortpflanzung, welche lediglich eine Funktion von Sexualität kennzeichnet (vgl. BZgA 2011: 18). Über den sogenannten Fruchtbarkeitsaspekt, welcher gekennzeichnet ist von einer Sehnsucht nach menschlichem Leben, hinaus beschreibt die $B Z g A$ deshalb vier weitere Aspekte, welche sich entweder nur auf das Individuum oder seinen (sexuellen) Austausch mit anderen Menschen beziehen. Die Funktion der sexuellen Befriedigung durch Masturbation beziehungsweise durch den sexuellen Kontakt mit anderen Menschen bezeichnet die Bundeszentrale als Lustaspekt. Im Beziehungsaspekt findet die Funktion von Sexualität einen Ausdruck für das Bedürfnis nach partnerschaftlichen Beziehungen zu anderen Personen mit sexueller Komponente. Mit dem von $\operatorname{der} B Z g A$ als Identitätsaspekt bezeichneten Lernprozess, wird die durch psychische, physische und soziale Einflüsse geprägte Entwicklung des Individuums beschrieben. Letztlich komplettiert der Kommunikationsaspekt die fünf Aspekte und Funktionen von Sexualität, da ein sexuelles Miteinander (sei es nun in Form von auf Dauer angelegten Beziehungen oder rein sexuellen Kontakten) ohne Kommunikation verbaler oder nonverbaler Art nicht möglich ist (vgl. BZgA 2004: 23f).

\section{Sexuelle Bildung}

Angelehnt an Sielert wird Sexuelle Bildung verstanden als lernzentrierte, alters- und entwicklungsspezifische sowie lebensphasenübergreifende Bildungs-, Beratungs- und Begleit(ungs)angebote zur Förderung der sexuellen Selbstbestimmung eines Menschen sowie der individuellen Sexualität. Im Zuge der sexuellen Sozialisation leisten entsprechende Bildungsangebote einen Beitrag in der nachhaltigen Befähigung von Menschen, sich vor Übergriffen und sexualisierter Gewalt zu schützen (vgl. Sielert 2015 (1): 28ff; vgl. Kapitel 3.1).

\section{Sexuelle Selbstbestimmung}

Unter sexueller Selbstbestimmung lässt sich nicht nur das Recht eines jeden Menschen fassen, über seine Sexualität selbst zu bestimmen, also frei zu entscheiden. Die gesellschaftlichen Auseinandersetzungen zu dieser Thematik schließen außerdem Diskussionen zum Recht auf Freiheit von sexualisierter Gewalt und die Entscheidungsfreiheit von Frauen in reproduktiven Fragen mit ein. Ebenfalls wird unter sexueller Selbstbestimmung 
das Recht, hinsichtlich seiner sexuellen Orientierung frei zu sei und darin anerkannt zu werden, gefasst (vgl. Deutscher Bundestag 2016).

\section{Sexualaufklärung und Sexualerziehung}

Durch Sexualaufklärung findet die Wissensvermittlung zu allen Aspekten von menschlicher Sexualität statt. In der (schulischen) Praxis beschränkt sie sich überwiegend auf eine Weitergabe von Informationen und findet meist in einem speziell dafür geschaffenen, einmaligen Rahmen statt. Sie versteht sich damit als Teil der Sexualerziehung, welche eine kontinuierliche Beeinflussung der sexuellen Entwicklung von Kindern und Jugendlichen beabsichtigt. Sexualerziehung bezieht dabei - über die reine Vermittlung von Fakten hinaus beispielsweise Auseinandersetzungen zu sexuellen Motivationen und Haltungsaspekte ein (vgl. Sielert 2013 (1): 41ff).

\section{Sexualpädagogik}

In der vorliegenden Arbeit wird die Sexualpädagogik als eine Teildisziplin der Pädagogik verstanden, welche die Sexualität von Menschen aller Altersgruppen in den Blick nimmt. Sie reflektiert dabei alle Aspekte von Sexualität, sexueller Sozialisation und Einflüsse von Sexualerziehung und erforscht diese hinsichtlich zahlloser Fragestellungen (vgl. ebd.).

\section{Sexualisierte Gewalt}

Unter sexualisierter Gewalt wird ein gesamtgesellschaftliches Phänomen von sexualisierten Übergriffen auf Kinder, Jugendliche und erwachsene Personen verstanden. Die Thematik ist seit etwa 30 Jahren mit verschiedenen Fokussen im Blickpunkt von Medien und Wissenschaft und wird je nach Kontext, Profession und entsprechender Fachdebatte beispielsweise als sexuelle Gewalt oder in Bezug auf Kinder und Jugendliche häufig als sexueller Missbrauch bezeichnet.

Konkret bezeichnet der Runde Tisch (vgl. Kapitel 1.3) sexuellen Missbrauch als psychologisch dadurch charakterisiert, dass eine übergriffige Person eine sexuelle Handlung an einem Kind vornimmt, wobei die Situation von einer mangelnden Einfühlung in das Kind gekennzeichnet ist (Runder Tisch 2011/2012: 11f). Sexuelle Handlungen sind dabei nicht exakt definiert und schließen je nach Betrachtungsweise alle - enge oder weite - als schädlich identifizierten Handlungen oder jedoch eindeutige sexuelle Körperkontakte zwischen Täter*in und betroffenem Kind ein (vgl. Arbeitsstab des UBSKM (3) o.J.). Weiterhin erklären die Expert*innen eine Abhängigkeitsbeziehung zwischen diesen Parteien als Merkmal der missbräuchlichen Situation, welche zur einseitigen Befriedigung von Macht- und anderen 
Bedürfnissen genutzt wird. Die Person, die die übergriffigen Handlungen ausführt, erlegt dem Kind in der Regel ein Geheimhaltungsgebot auf und nutzt letztlich die zwiespältigen Gefühle des jungen Menschen in Bezug auf die Situation und das Verhältnis zumzur Täter*in aus (vgl. Runder Tisch 2011/2012: 11f).

\subsection{Historische Ausgangslage}

Das Merseburger Forschungsprojekt „Schutz von Kindern und Jugendlichen vor sexueller Traumatisierung “resultiert aus einer Vielzahl von Aktivitäten, die die Bundesregierung im Zuge der Aufdeckung zahlreicher institutioneller Missbrauchsfälle vorangetrieben hat: Bereits seit Mitte der 90er Jahre wurden vereinzelte Vorfälle sexualisierter Gewalt in Institutionen - in diesem Fall insbesondere in Einrichtungen der römisch-katholischen Kirche - bekannt (vgl. Langer 2015). Auf Initiative des Rektors des Canisius-Kollegs der Jesuiten in Berlin, Pater Klaus Mertes, kam es in Deutschland Anfang 2010 schließlich zu einer Aufdeckungswelle sexueller Übergriffe in Internaten und kirchlichen Räumen, die noch im selben Jahr den Beschluss und kurze Zeit danach auch die erste Zusammenkunft eines sogenannten Runden Tisches nach sich zog (vgl. Langer 2015). Am Runden Tisch fanden sich zahlreiche Vertreter*innen des Bundesministeriums für Familie, Senioren, Frauen und Jugend (BMFSFJ), des Bundesministeriums der Justiz (BMJV) sowie des Bundesministeriums für Bildung und Forschung (BMBF) sowie zahlreiche namenhafte Familienverbände, Beschäftigte von Schulund Internatsträgern, Abgeordnete der einzelnen Bundesländer und Vertreter*innen der katholischen und evangelischen Kirche. Ebenso brachten sich Sexualwissenschaftler*innen sowie Mediziner*innen, Psycholog*innen und Gesandte der entsprechenden Berufsverbände als Teilnehmende ein. Ergebnis zweier Tagung im Jahr 2010 und 2011 des Runden Tisches „Sexueller Kindesmissbrauch in Abhängigkeits- und Machtverhältnissen in privaten und öffentlichen Einrichtungen und im familiären Bereich" war ein 267 Seiten umfassender Abschlussbericht, welcher sich neben grundsätzlichen Definitionen insbesondere damit auseinandersetzt, wie gegenwärtig gehandelt werden sollte, um zum einen die Aufarbeitung der bekannten und der noch nicht aufgedeckten Fälle voranzutreiben, zum anderen aber auch Kinder und Jugendliche zukünftig effektiver vor sexualisierter Gewalt zu schützen (vgl. Langer 2015). Der Runde Tisch stellte insbesondere heraus, dass es bis dato wenig empirisch belegbare und wissenschaftlich fundierte Kenntnisse zu sexuellem Missbrauch vorhanden sind (vgl. Runder Tisch 2011/2012: 3, 53). Weiterhin bildet in den Empfehlungen des Abschlussberichtes neben der Stärkung der Heranwachsenden und ihrer Eltern die Qualifizierung von Fachkräften einen der Schwerpunkte. Ziel sollte sein, diese perspektivisch dazu zu befähigen, pädagogische Einrichtungen so auszustatten, dass sie sichere Räume sein können. Sogenannte Schutzkonzepte bilden die Grundlage für eine derart sichere Umgebung in pädagogischen Einrichtungen (vgl. ebd.). 
Neben dem Runden Tisch wurde im März 2010 zeitgleich das Amt der ersten UBSKM einberufen, welches die damalige Bundesfamilienministerin Christine Bergmann besetzte. Ihr Tätigkeitsschwerpunkt sollte in der Aufarbeitung der institutionellen Fälle sexualisierter Gewalt sowie in der Stärkung der Rechte der Betroffenen liegen (vgl. Aktionsstab des Unabhängigen Beauftragten für Fragen des sexuellen Kindesmissbrauchs (1) o.J.). Auch die UBSKM veröffentlichte 2011 einen umfangreichen Abschlussbericht, in welchem sie zum einen Ergebnisse der Aufarbeitung bekannt gab, aber auch konkrete Handlungsempfehlungen zum Schutz von Kindern und Jugendlichen vor sexualisierter Gewalt aussprach (vgl. ebd.). Eine zusätzliche Ergänzung zum Runden Tisch und dem Amt der UBSKM stell der Aktionsplan 2011 der Bundesregierung zum Schutz von Kindern und Jugendlichen vor sexueller Gewalt und Ausbeutung dar. Neben Prävention und Intervention identifizierte der Aktionsplan den Bereich Wissen als eines von sieben Handlungsfeldern. Maßgeblich sollten dafür umfangreiche Forschungsmaßnahmen sowie ein gelingender Theorie-Praxis-Transfer sein (vgl. BMFSFJ 2011).

$\mathrm{Zu}$ allen drei Initiativen entschloss sich die Bundesregierung um Kindern und Jugendlichen zukünftig umfassend vor sexueller Gewalt zu schützen. Erstmalig legte sie damit einen derart umfangreichen Schwerpunkt auf die Förderung von Forschungsvorhaben in diesem Bereich. Um die Forschung im Feld voranzubringen und eine nachhaltige Implementierung der Forschungsergebnisse zu sichern, investierte das BMBF seit 2011 rund 63 Millionen Euro und förderte Forschungsvorhaben, welche „sowohl fundierte Erkenntnisse zu den strukturellen und personalen Faktoren von sexualisierter Gewalt als auch zu Fragen der Prävention zur Verfügung stellen“ (Runder Tisch 2011/2012: 257). Insbesondere die Erforschung struktureller Bedingungen für institutionelle sexualisierte Übergriffe, die Weiterentwicklung von präventiven Maßnahmen sowie die Deskription und Evaluation vorhandener Kompetenzen im Umgang mit sexualisierter Gewalt und entsprechender Fortbildungsangebotene waren inhaltliche Förderungsgegenstände für potentielle Forschungsinteressen. Um eine „Kultur des Hinsehens" zu etablieren und Fachkräfte im Umgang mit Fragen zu sexuellen Kindesmissbrauch zu professionalisieren, wurden ebenfalls Forschungsprojekte gefördert, die auf Kompetenzerweiterung von pädagogisch Tätigen sowie deren Aus- und Weiterbildung abzielten. Angesiedelt sind beziehungsweise waren 22 dieser Forschungsvorhaben (einige sind inzwischen bereits abgeschlossen) in der Förderrichtlinie „Forschung zur Prävention sexueller Gewalt in pädagogischen Kontexten“.

Weiterhin sah der Abschlussbericht des Runden Tisches die Etablierung von insgesamt fünf Junior- und Forschungsprofessuren an Hochschulen vor, welche ebenfalls eine dauerhafte Aufmerksamkeit des Themenfeldes im wissenschaftlichen Kontext sicher sollten (vgl. ebd.). Eine der fünf Professuren startete im Mai 2014 an der Hochschule Merseburg. Sie „zielt auf die nachhaltige Verankerung von Forschung im deutschlandweit einzigartigen Lehr- und 
Forschungsbereich Angewandte Sexualwissenschaften“ (BMBF 2015: 54). Das ebenfalls vom $B M B F$ geförderte Forschungsprojekt „Schutz von Kindern und Jugendlichen vor sexueller Traumatisierung" ist der Forschungsprofessur angesiedelt. Zeitgleich mit der Forschungsprofessur begann die erste Förderphase des Forschungsprojektes im Mai 2014, welche nach 36 Monaten im Mai 2017 in die zweite Förderphase überging. Nach inzwischen vierjähriger Laufzeit wurden Erhebungsinstrumente entwickelt, Förderschwerpunkte pointiert und erste Ergebnisse umgesetzt. In den übrigen zwei Jahren Laufzeit wird das Forschungsteam an die Ergebnisse der ersten Förderphase anknüpfen und zentrale Zielstellungen konkretisieren und weiterverfolgen.

Alle Bemühungen der Bunderegierung, den Schutz von Kinder und Jugendlichen vor sexualisierter Gewalt zu stärken, zu verbessern und zu erhalten, mündeten 2014 schließlich in ein Gesamtkonzept. Es vereint die Handlungsempfehlungen des Runden Tisches und die Aktivitäten des Aktionsplans 2011 und regelt die Zusammenarbeit aller Akteure im Feld (vgl. Internetredaktion des BMFSFJ 2017).

\subsection{Empirische Ausgangslage}

Im Folgenden soll die empirische Ausgangslage, also die Inhalte, Methodik und Ergebnisse der ersten Förderphase des Projekts „Schutz von Kindern und Jugendlichen vor sexueller Traumatisierung" erläutert werden. Da diese Aspekte Grundlage der eigenen Empirie dieser Masterarbeit sind, betten sie sich in ein Gesamtverständnis des Forschungsvorhabens. Sie werden gerahmt von einem Ausblick auf die bereits laufende zweite Förderphase des Projekts.

\section{Inhalte}

Im Fokus des oben genannten Forschungsprojektes stand und steht seit Beginn der ersten Förderphase, diejenigen Faktoren zu analysieren und evaluieren, welche dazu beitragen, Kinder und Jugendliche in ihrer (sexuellen) Selbstbestimmung zu fördern und zu stärken. Hintergrund dessen ist die Idee, dass (sexuell) selbstbestimmt lebende junge Menschen aufgrund ihrer psychischen und personellen Konstitution besser vor sexualisierter Gewalt und/oder Traumatisierung durch entsprechende Vorfälle geschützt sind (vgl. BMBF 2015: 54). Zur Verfolgung dieser Grundüberlegung formulierte das Forschungsteam der Hochschule Merseburg drei konkrete Fragen, die die Richtung der Forschungsarbeit vorgeben:

Wie kann sexuelle Aufklärung der Prävention sexueller Gewalt dienen?

Wie ist durch Sexualpädagogik und Sexuelle Bildung eine verbesserte sexuelle Selbstbestimmung erreichbar, die die Abwehr potenzieller und die Verarbeitung bereits bestehender sexueller Grenzverletzung erleichtert? 
Welche Bedingungen personeller und institutioneller Art wirken an pädagogischen Einrichtungen (und in der Lebenswelt von Kindern und Jugendlichen) förderlich für sexuelle Selbstbestimmung?

(BMBF 2015: 54)

Wie aus den Fragen erkennbar, setzt das Wirken des Forschungsprojektes nicht unmittelbar bei den Kindern und Jugendlichen selbst an. Vielmehr entschieden sich die Projektmitarbeiter*innen dazu, die Bedarfe von Fachkräften hinsichtlich sexualpädagogischer Fort- und Weiterbildungen zu erheben und sich einen Überblick darüber zu verschaffen, (in)wie(fern) kompetent pädagogisch Tätige bereits jetzt im entsprechenden Themenfeld in der Arbeit mit Klient*innen sind (vgl. ebd.). In den Fokus der Erhebung wurden Fachkräfte der ambulanten Erziehungshilfen, insbesondere der Sozialpädagogischen Familienhilfen (SpFh) und Einzelbetreuungen/Erziehungsbeistandschaften (EB), gesetzt. Zum einen fand dieser Bereich bisher kaum Beachtung in wissenschaftlichen Untersuchungen. Zum anderen nimmt er jedoch bundesweit in der Kinder- und Jugendhilfe einen enormen Stellenwert im Rahmen der ambulanten Hilfen ein, wie man den Informationen des Statistischen Bundesamtes für die Jahre 2015 und 2016 entnehmen kann:

\begin{tabular}{|c|c|c|}
\hline Hilfearten & 2015 & 2016 \\
\hline \multicolumn{3}{|l|}{ Ambulante Hilfen } \\
\hline \multicolumn{3}{|l|}{ Hilfen nach $\S 27$ SGB VIII ${ }^{1}$} \\
\hline begonnene Hilfen & 30497 & 33136 \\
\hline beendete Hilfen & 29851 & 30168 \\
\hline Hilfen am 31.12 . & 41278 & 42302 \\
\hline \multicolumn{3}{|l|}{ Erziehungsberatung } \\
\hline begonnene Hilfen & 305922 & 306164 \\
\hline beendete Hilfen & 305535 & 303289 \\
\hline Hilfen am 31.12 . & 141825 & 145404 \\
\hline \multicolumn{3}{|l|}{ Soziale Gruppenarbeit } \\
\hline begonnene Hilfen & 7100 & 7056 \\
\hline beendete Hilfen & 7105 & 6849 \\
\hline Hilfen am 31.12. & 9197 & 9009 \\
\hline \multicolumn{3}{|l|}{ Einzelbetreuung ${ }^{2}$} \\
\hline begonnene Hilfen & 27076 & 29339 \\
\hline beendete Hilfen & 26210 & 27115 \\
\hline Hilfen am 31.12 . & 30243 & 31698 \\
\hline \multicolumn{3}{|l|}{ Sozialpädagogische Familienhilfe } \\
\hline begonnene Hilfen & 45379 & 46941 \\
\hline Zahl der Kinder & 82586 & 87361 \\
\hline beendete Hilfen & 44543 & 44208 \\
\hline Zahl der Kinder & 83022 & 83347 \\
\hline Hilfen am 31.12. & 71525 & 71846 \\
\hline Zahl der Kinder & 137746 & 139400 \\
\hline
\end{tabular}

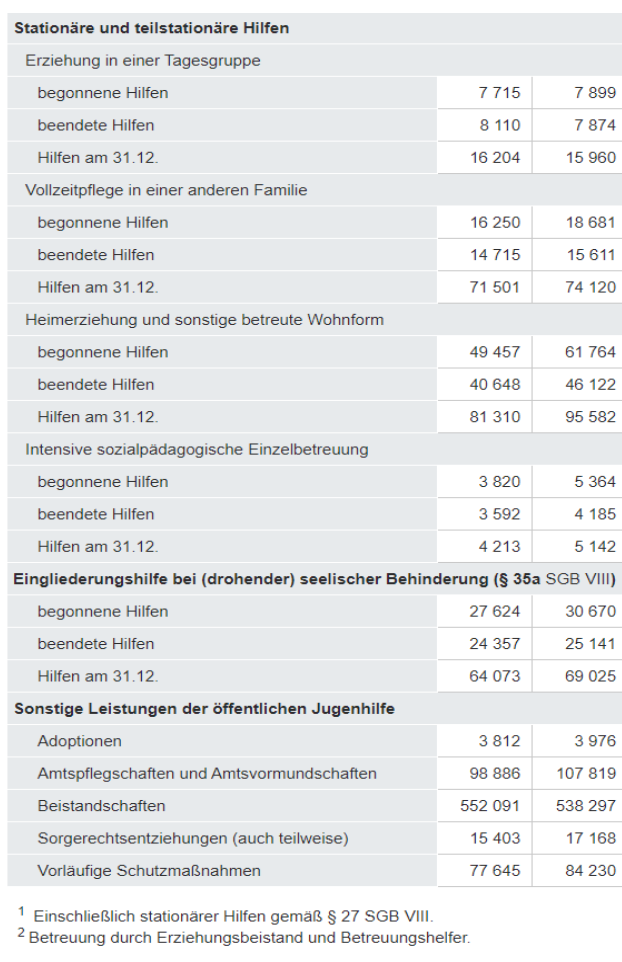

Abb. 2: Erzieherische Hilfen und sonstige Leistungen, Teil 2

Quelle: Statistisches Bundesamt 2017.

In der Statistik werden für das Jahr 2016 insgesamt 516.344 begonnene Hilfen ausgewiesen, von denen 422.636 Fälle den ambulanten Erziehungshilfen zuzuordnen sind. Dies entspricht 
einem prozentualen Anteil von $77,3 \%$ der Gesamthilfenanzahl. Allein die SpFhs und EB summieren sich auf ein Fünftel der 2016 gestarteten nichtstationären Interventionen und stellen damit einen sehr wesentlichen Bestandteil dieser dar.

Über die pädagogischen Fachkräfte in ambulanten Einrichtungen hinaus identifizierte das Forschungsteam auch Lehrkräfte schulischer Institutionen als potentiell relevante Zielgruppe, um zur Beantwortung der Merseburger Forschungsfragen beizutragen. Aus diesem Grund wurden im kleinerem Umfang auch im Schulbetrieb tätige Personen in die Erhebung einbezogen (vgl. Voß et. al 2014: 5).

\section{Methodik und Untersuchungsvorgehen}

Zur qualitativen Datenerhebung wurden im Forschungsprojekt leitfadengestützte, halboffene Interviews geführt (vgl. Anhang: iff). Die Stichprobe hatte eine Größe von n=43, wovon 19 Interviews mit ambulant betreuenden Sozialpädagog*innen geführt wurden, sechs Gespräche mit Lehrer*innen stattfanden und die übrigen 18 mit intersektional arbeitenden Fachkräften erfolgten. Auf einen offenen Erzählimpuls - der Frage danach, welche Berührungspunkte es in der täglichen Arbeit mit dem Thema Sexualität gäbe - folgten in der Regel Nachfragen durch die interviewende Person zu den bis dahin aufgekommenen Inhalten. Anschließend orientierte sich der ${ }^{\star}$ die Interviewer ${ }^{\star}$ in an verschiedenen Themenblöcken entsprechend des Fragebogens und gab Möglichkeiten zum Austausch über Grenzverletzungen und den üblichen Umgang in der Einrichtung, Erfahrungen mit Fort- und Weiterbildungsangeboten und Wünschen für zukünftige Besuche derselben, dem Verständnis und einrichtungsspezifischen Angeboten von Sexueller Bildung und ähnlichem (vgl. Krolzik/Matthei/ et. al 2018: 249f).

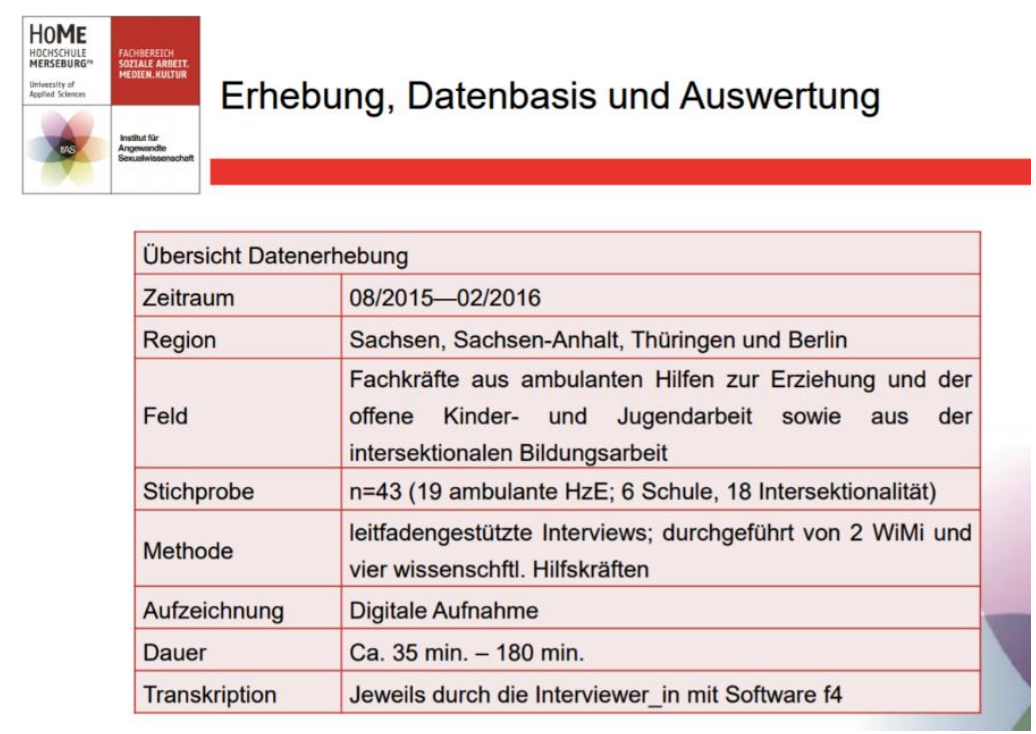

Abb. 1: Übersicht Datenerhebung

Quelle: Krolzik-Matthei/Linke 2017: 5. 
Im weitere Verlauf der ersten Förderphase erfolgte mittels Qualitativer Inhaltsanalyse nach Mayring und der Software MaxQDA eine erste Auswertung des Materials. Wie von Mayring vorgesehen (vgl. Kapitel 5.6.1), wurden zunächst deduktive Kategorien formuliert, nach denen das vorhandene Datenmaterial analysiert wurde. Im weiteren Verlauf folgte die Aufstellung zusätzlicher induktiver Kategorien und insbesondere die Untersuchung des Materials darauf, wie die befragten Personen Sexualität während des Interviews thematisieren. Ausgehend davon erfolgten Interpretationen des Gesagten bezüglich förderlichen Bedingungen einer ambulanten Hilfe zur Erziehung, um Kinder und Jugendliche in ihrer sexuellen Selbstbestimmung zu stärken (vgl. Krolzik-Matthei et. al 2018: 249f).

Um die Interviewreihe der ambulant tätigen Sozialarbeiter*innen sinnvoll zu ergänzen, wurden außerdem Interviews mit Lehrer*innen durchgeführt, die im Rahmen der Auswertung innerhalb des Forschungsprojektes aus verschiedenen Gründen keine Berücksichtigung finden konnten. Die Interviews mit den Lehrkräften sind Grundlage der vorliegenden Arbeit und werden im weiteren Verlauf entsprechend methodisch betrachtet (vgl. Kapitel 5, vgl. Kapitel 6).

Die erste Förderphase des Merseburger Forschungsvorhabens war zudem von einer inhaltlichen Zweiteilung gekennzeichnet. Während ein Erhebungsschwerpunkt auf den oben beschriebenen Erziehungshilfekontext zielte, fokussierte das zweite Teilprojekt auf intersektional tätige Einrichtungen und deren Erfahrungen mit Grenzverletzungen, Sexualität und sexualisierter Gewalt. Auch dort diente die Untersuchung dazu, Informationen über die Tätigkeit der Institutionen, vor allem aber zu den Bedarfen an Fort- und Weiterbildung in besprochenen Themenbereichen zu erhalten (vgl. Krolzik-Matthei/Linke 2017: 5).

\section{Ergebnisse}

Die Ergebnisse der Auswertungen in den ersten 36 Monaten der Projektlaufzeit sind vielgestaltig und beinhalten eine Reihe verschiedener Perspektiven. Zunächst wurde der Bereich der SpFh/Erziehungsbeistandschaft als ein Feld der Sozialen Arbeit identifiziert, in welchem besondere Machtverhältnisse wirken (vgl. Krolzik-Matthei et. al 2018: 251f). Als ursächlich dafür zeigt sich überwiegend die Hürde, zentrale Aufträge für alle Personen im Hilfeprozess klar zu benennen und zuzuweisen. Insbesondere verdeckte Aufträge, die sich im Konstrukt öffentlicher Träger (ASD), hilfeerbringende Stelle (SpFh/EB) und Familie häufig in der Form zeigen, dass sich die SpFh Aufträge des ASDs annimmt, welche weder im Hilfeplan festgehalten sind noch mit der betroffenen Familie kommuniziert worden, können eine ambulante Hilfe zu einem vielschichten Aushandlungsfeld von Macht werden lassen. Gibt es keinen, den Umgang mit der Thematik regelnden, offen besprochenen Auftrag des Jugendamtes und somit auch keine zielgerichtete Bearbeitung des Themenbereiches, erhöht sich zwangsläufig die Wahrscheinlichkeit, dass jede der beteiligten Parteien im eigenen Interesse handelt. Dies kann zu verschiedenen Szenarien führen und eine 
ganze Bandbreite an Möglichkeiten abdecken. Denkbar sind beispielsweise Situationen, die von völliger Ausgrenzung des Themas trotz Signalisierung eines entsprechenden Bedarfs über den Versuch, erzwungene Gespräch über Sexualität als Ermächtigung zu nutzen, reichen und letztlich auch in der Suggestion subjektiver Wertvorstellungen münden, mit denen Fachkräfte versuchen könnten, die Klient*innen in eine vermeintlich angemessene Richtung zu lenken. Die (bewusste oder unbewusste, geplante oder ungeplante) Absicht, das Themenfeld Sexualität als Mittel für eigene Zwecke zu nutzen, kann dabei sowohl von Klient*innen als auch der Fachkraft ausgehen. Die Scham, mit der die Thematik Sexualität häufig gesellschaftlich besetzt ist, erschwert die detaillierte Auftragsklärung zusätzlich (vgl. Krolzik-Matthei et. al 2018: 251f).

Neben Sexualität als Aushandlungsfeld von Macht zeigte sich in den Interviewauswertungen insbesondere der Umgang mit sexualisierter Gewalt als weiterer Schwerpunkt. Zunächst offenbarte sich in der Analyse ein hohes Maß an Vulnerabilität aufseiten der Fachkräfte, wenn Fälle von sexualisierter Gewalt im Hilfeprozess zu Tage treten (vgl. Krolzik-Matthei/Linke 2017: 8ff). Dies schließt sowohl ein schon vor Hilfebeginn bekanntes Vorhandensein entsprechender Geschehnisse ein, als auch die Möglichkeit, dass derartige Aufdeckungen erst während der laufenden Hilfe bekannt (gemacht) werden. Aufgrund der besonderen Konstellation im

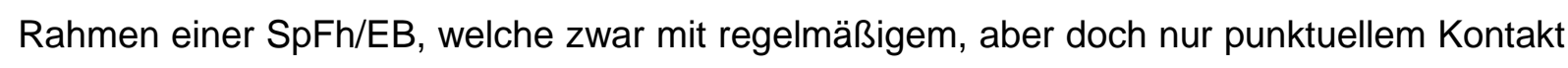
einhergeht, sind Fachkräfte häufig unsicher in ihren Interventionen bei Aufdeckung oder Bekanntwerden von Fällen sexualisierter Gewalt. Weitere Faktoren, die das begünstigen, sind der nahezu immer vorherrschende Mangel an Ressourcen (in der SpFh ist dies vermehrt die Ressource "Zeit“ sowohl im Kontakt mit der Familie als auch bezüglich möglicher Weiterbildungen) sowie die Breite an Themenfelder, in denen die Fachkraft kompetent sein muss, um die Klient*innen adäquat zu begleiten. Die Notwendigkeit, als professionell helfende Person trotz Unsicherheit oder Unwissen in irgendeiner Form handeln zu müssen, bringt die Sozialpädagog*innen oftmals in hochbelastende Situationen, die von innen als Dilemmata empfunden werden (vgl. Institut für Angewandte Sexualwissenschaften 2018). Neben Supervision, die innerhalb der Interviewauswertung als wichtige und notwendige Intervention identifiziert wurde, zeigte sich weiterhin, dass Fachkräfte zusätzliche Angebote brauchen, um den Umgang mit Sexualität und sexualisierter Gewalt innerhalb der SpFh/EB zu professionalisieren (vgl. ebd.: 10ff).

Bereits im ursprünglichen Forschungsdesign nahmen die Projektmitarbeitenden an, dass ein Nutzen für Praktiker*innen perspektivisch darin liegen könnte, sich vermehrt in Netzwerken zu beteiligen und Sicherheit im Umgang mit Sexualität und angrenzenden Themen wie sexualisierter Gewalt und (sexuellen) Grenzverletzungen zu erlangen (vgl. Voß et. al 2014: 22ff). Dass ein entsprechender Bedarf auf Seiten der in der ambulanten Jugendhilfe Tätigen 
tatsächlich vorliegt, wurde durch die Auswertung der Interviews verifiziert (vgl. Institut für angewandte Sexualwissenschaften 2017).

\section{Ausblick auf die zweite Förderphase}

Aktuell befindet sich das Forschungsprojekt „Schutz von Kindern und Jugendlichen vor sexueller Traumatisierung" in der zweiten Förderphase. Diese begann im Mai 2017 und wird nach 36-monatiger Laufzeit im April 2020 enden.

Wie bereits in den ersten drei Jahren Laufzeit fokussiert das Forschungsvorhaben weiterhin auf Handreichungen und Materialentwicklung, um Fachkräfte der ambulanten Erziehungshilfen künftig in ihren Kompetenzen im Umgang mit den Themen Sexualität und sexualisierte Gewalt zu stärken. Zusätzlich dazu liegt ein weiterer Projektschwerpunkt für diese Forschungsförderlinie auf dem Theorie-Praxis-Transfer.

Der vom Merseburger Forschungsprojekt in den ersten drei Jahren Laufzeit im Zuge der Interviewauswertungen nachgewiesene Bedarf an Professionalisierung im Umgang mit sexualisierter Gewalt in ambulanten Hilfekontexten ist ein wesentlicher Anknüpfungspunkt für besagten Theorie-Praxis-Transfer. Aufbauend auf von Fachkräften formulierten Bedarfen wurde das Konzept für einen Regionalen Arbeitskreis "Sexualisierte Gewalt" (RAK) entwickelt, welcher - initiiert vom Forschungsteam - perspektivisch Möglichkeiten bieten soll, die Handlungskompetenzen der Sozialarbeiter*innen zu stärken (vgl. Institut für Angewandte Sexualwissenschaften 2017).

Neben dem Anstoß zur Gründung des RAKs komplettieren Interviews mit Jugendlichen, die Auswertung derselben und die Veröffentlichung von Forschungsergebnissen das Vorhaben der zweiten Förderphase (vgl. Voß et. al 2014: 13ff).

\subsection{Forschungsethische Ausgangslage}

Unter Beachtung der sensiblen Thematik veröffentlichten Poelchau et al. im Jahr 2015 erstmalig in Deutschland eine forschungsethische Übereinkunft mit konkreten Empfehlungen für Forschungsvorhaben zu sexualisierter Gewalt in pädagogischen Kontexten. Einerseits soll die Bonner Ethik-Erklärung eine Orientierungshilfe für Forschende sein, um adäquat mit ethischen Fragestellungen und rechtlichen Aspekten bezüglich ihres Forschungsfeldes umgehen zu können. Mit der Handlungssicherheit für die Wissenschaftler*innen trägt sie maßgeblich zum Schutz des Menschenwohls und der Menschenrechte bei. Sie gibt konkrete Hinweise darauf, wie Risiken für die beforschten Individuen möglichst minimal gehalten und Forscher*innen mit Blick auf die spezifischen Anforderungen geschult werden können (vgl. Poelchau et. al 2015: 1ff). 
Im Forschungsprojekt „Schutz von Kindern und Jugendlichen vor sexueller Traumatisierung“ wird der Bonner Ethikerklärung an verschiedenen Punkten Rechnung getragen. So werden alle Interpartner*innen stets darüber aufgeklärt, dass die Teilnahme am Interview freiwillig ist und jederzeit unter- oder abgebrochen werden kann. Eine für beide Seiten verbindliche Sicherheit stellt dabei eine von der befragten Person zu unterzeichnende Erklärung dar (vgl. Anhang: $x$ ). Diese besagt, dass sie im Vorfeld über die Hintergründe ebenso wie über die Bedingungen des Interviews (Freiwilligkeit, Anonymisierung, Verwendung der Daten etc.) aufgeklärt worden ist.

Ein weiteres forschungsethisch besonderes Augenmerk liegt auf dem Umgang mit den erhobenen Daten. Mit Hilfe eines Pseudonymisierungs- und Anonymisierungsprozesses wird sichergestellt, dass von Fachkräften in Interviews getroffene Aussagen im Rahmen von Ergebnisveröffentlichungen nicht auf konkrete Personen oder Institutionen zurückführbar sind. Interviewpassagen, die wissenschaftliche Erkenntnisse in Zitatform unterstreichen, werden lediglich mit einem kurzen Code kenntlich gemacht und bieten damit Außenstehenden keine Möglichkeit, etwaige Rückschlüsse zu ziehen (vgl. Voß et. al 2014: 16ff).

Darüber hinaus wird bereits im Forschungsdesign ein sensibler Umgang mit möglichen Risiken innerhalb des Forschungsvorhabens ausgewiesen. Neben einer Interviewer*innenschulung zu den Themengebieten Traumata, sexualisierter Gewalt und Umgang mit belastenden Situationen innerhalb der Interviewsituation und vom Datenschutzbeauftragten der Hochschule vermittelte Informationen zu den Datenschutzbestimmungen begleiten regelmäßige Supervisionstermine das Forschungsgeschehen. 


\section{Forschungsvorhaben}

Im 2. Kapitel soll das Forschungsvorhaben dieser Masterthesis dezidiert erläutert werden. Zunächst wird hierfür das eigentliche Forschungsinteresse näher beschrieben. Das Forschungsvorhaben ist inhaltlich dem Merseburger BMBF-Forschungsprojekt zuzuordnen (vgl. Kapitel 1.4). Aufgrund dessen ist auch das hier formulierte Forschungsinteresse eng mit den Projektinhalten verknüpft. Der konkrete Forschungsgegenstand und die zu dessen Untersuchung formulierten Forschungsfragen orientieren sich deshalb ebenfalls an den zentralen Forschungsfragen des BMBF-Projekts.

\subsection{Forschungsinteresse}

Das der Arbeit zugrundeliegende Forschungsinteresse fokussiert auf die Frage, welchen Beitrag Lehrkräfte leisten, leisten müssten und aktuell leisten können, um Kinder und Jugendliche im Lebensraum Schule vor sexualisierter Gewalt zu schützen und sie in ihrer positiven Entwicklung und sexuellen Selbstbestimmung zu stärken.

Zentral im Forschungsvorhaben sind dabei momentane Facetten Sexueller Bildung in der Institution Schule und die Kompetenzen von Lehrkräften, die sie während des Lehramtsstudiums und durch Fort- und Weiterbildung erworben haben.

Als wesentliche Sozialisationsinstanz hat die Institution Schule einen großen Einfluss auf die (Persönlichkeits-)Entwicklung von jungen Menschen (vgl. Hummrich/Kramer 2017: 7f). In allen zentralen Sozialisationsinstanzen findet im Einklang mit der persönlichen Entwicklung von Heranwachsenden auch die sexuelle Sozialisation statt. Der Schule kommt dabei eine besondere Bedeutung zu. Durch die in Deutschland geltende Schulpflicht ist die Schule für alle in Deutschland lebenden Kinder und Jugendliche der einzige Ort, an dem sie zwangsläufig in einem institutionellen Kontext mit Aspekten von Sexualität in Berührung kommen. Diese sind vielschichtig und reichen beispielsweise von Kontakten zu Mitschüler*innen und Lehrpersonal bis hin $\mathrm{zu}$ unterrichtlicher und außerunterrichtlicher schulischer Auseinandersetzung mit sexuellen Themen. Lehrkräfte als unmittelbare Bezugs-, Autoritäts-, und Orientierungspersonen haben mit ihrem Handeln, ihren eigenen Haltungen und Wertvorstellungen sowie ihrem professionellen Selbstverständnis einen bedeutsamen Einfluss auf die sexuelle Sozialisation der Heranwachsenden. Umso wichtiger ist es, dass es innen gelingt, das eigene Agieren zu reflektieren und geeignete Voraussetzungen dafür zu schaffen, dass Kinder und Jugendliche sich frei von Zwängen in einem positiven Umfeld entwickeln können (vgl. Schmidt 2014: 254ff, vgl. Kapitel 3).

Das zugrundeliegende Forschungsinteresse setzt damit unmittelbar am Forschungsvorhaben des vom BMBF geförderten und an der Hochschule Merseburg angesiedelten Projektes „Schutz von Kindern und Jugendlichen vor sexueller Traumatisierung“ an. 
Mit Hilfe von Forschungsfragen soll die Auswertung qualitativer Interviews dazu beitragen, sich mit dem im Folgenden konkret formulierten Forschungsgegenstand auseinander zu setzen und das Forschungsinteresse $\mathrm{zu}$ verfolgen. Relevant sind dabei insbesondere Untersuchungen zu Kompetenzen der Lehrkräfte, ihrem Reflexions- und Handlungsvermögen und den Möglichkeiten, die sie haben, um im schulischen Rahmen wirksam die sexuelle Sozialisation von Kindern und Jugendlichen positiv zu beeinflussen.

\subsection{Forschungsgegenstand und Forschungsfragen}

Der Forschungsgegenstand, welchem sich die vorliegende Untersuchung widmet, beschreibt die Erfahrungen und Kompetenzen von Lehrkräften an allgemeinbildenden Schulen im Kontext von Sexualität beziehungsweise sexualisierter Gewalt. Dieser Gegenstand wird zum einen mit einer theoretischen Auseinandersetzung, zum anderen mit einer qualitativen Untersuchung erforscht.

Um sich dem eigentlichen Forschungsinteresse zu nähern und den Forschungsgegenstand zu untersuchen, wurden Forschungsfragen formuliert. Diese eröffnen unterschiedliche Perspektiven der Betrachtung von Sexualität im Kontext der Institution Schule sowie den Umgang mit der Thematik sexualisierte Gewalt durch Lehrkräfte. Die formulierten Forschungsfragen lauten wie folgt:

- Welchen Beitrag leistet die Institution Schule dazu, Kinder und Jugendliche durch Prävention vor sexualisierter Gewalt und/oder ihren Folgen zu schützen?

- Über welche Kompetenzen verfügen Lehrkräfte im Hinblick auf Sexuelle Bildung ihrer Einschätzung nach?

- Welche Möglichkeiten haben Lehrkräfte im Rahmen des Schulalltags, Kinder und Jugendliche in ihrer sexuellen Selbstbestimmung zu stärken?

- Welche Bestandteile der Aus- und Fortbildung von Lehrkräften befähigen sie zu einem professionellen Umgang mit dem Themenfeld Sexualität im Schulalltag?

Insbesondere bei der Erstellung des Kategoriensystems im Rahmen der qualitativen Inhaltsanalyse nach Mayring (vgl. Kapitel 5.4) sollen die Forschungsfragen auf konkrete Themenbereiche eingrenzen. Weiterhin sollen sie unterstützend dafür sein, das vorhandene Material mit verschiedenen Fokussen $\mathrm{zu}$ betrachten und so einen differenzierteren Erkenntnisgewinn begünstigen. 


\section{Sexuelle Bildung in Schulen - Theoretischer Kenntnisstand}

Die Lehrpläne aller Bundesländer sehen vor, dass an Schulen Sexualaufklärung geleistet werden muss (vgl. Hilgers 2004. 9). Dies soll insbesondere vor dem Hintergrund der Aufklärung selbst geschehen, aber auch einen Beitrag dazu leisten, präventiv gegen sexualisierte Gewalt an Kindern und Jugendlichen zu wirken. Im Hinblick darauf, dass Sexuelle Bildung deutlich umfassender ist, tiefgreifender ansetzt und über eine reine Wissensvermittlung hinauswirken will (vgl. Kapitel 3.2), ist sie von den Bildungsangeboten, die Kindern und Jugendlichen aktuell häufig an Schulen gemacht werden, abzugrenzen. Im Weiteren soll deshalb überwiegend der Begriff „schulische Sexualerziehung“ und gelegentlich „schulische Sexualaufklärung“ Anwendung finden.

Es bietet sich zunächst an, die historischen Hintergründe näher zu betrachten.

\subsection{Historische Ausgangslage Sexueller Bildung an Schulen}

\section{Schulische Sexualerziehung bis 1968}

Bis zum Jahr 1968 gibt es in der Bundesrepublik Deutschland nur vereinzelte Bestrebungen der Länder, Sexualerziehung in schulischen Institutionen umzusetzen. Mit gutem Beispiel voran gehen bis dahin Bundesländer wie Berlin, Hamburg und Hessen, welche zum Teil flächendeckend, zum Teil als Empfehlung die Aufgabe an Lehrkräfte herantragen, den Schüler*innen Inhalte zu Sexualität und Aufklärung zu vermitteln (vgl. Hilgers 2004: 9). Gesamtgesellschaftlich ist um das Jahr 1968 ein politischer und kultureller Wandel zu verzeichnen. Am deutlichsten spiegelt sich dies in den studentischen Protestbewegungen in den Jahren 1967-1969 wider (vgl. Sager 2015: 126). Sager formuliert dazu, dass die Entwicklung zu diesem Zeitpunkt „mit einer Westernisierung einher [geht] und (...) in diesem Sinne auch als Katalysator auf dem Weg in eine moderne Gesellschaft verstanden werden [kann]“ (ebd.: 126). Parallele Entwicklungen weltweit tragen ihr Übriges dazu bei und ermöglichen beziehungsweise begünstigen internationale Kooperationen zwischen Studierenden, aber auch wissenschaftlich tätigen Personen. Zentraler Bestandteil der gesellschaftlichen Entwicklung und derer, die sie zu diesem Zeitpunkt maßgeblich voranbringen, ist die Abgrenzung der eigenen, jüngeren Generation zur älteren und deren Involvierung in die nationalsozialistischen Strukturen der vergangenen Jahrzehnte (vgl. ebd.: 126, 128). Im Bewusstsein darüber, dass die neuen Ideale nicht mehr durch die vorherige Generation mit ihren Verfehlungen umgesetzt werden können, rückt die nächste Generation in den Fokus der Protagonist*innen. Wie Sager weiter ausführt, hat ihr Bestreben aus diesem Grund - anders als in anderen Ländern - im deutschen Raum eine wesentliche pädagogische Komponente und verhandelt eine Vielzahl an pädagogischen Fragestellungen (vgl. ebd.: 127). Primäres Ziel war es, durch grundlegend neue erzieherische Impulse Kinder von negativen 
gesellschaftlichen Einflüssen zu schützen und sie als Mitbegründer*innen einer neuen Gesellschaft in ihrer Entfaltung zu fördern und zu stärken. Entsprechend nah liegt die Einbeziehung eines neuen Umgangs mit Fragen zu (kindlicher) Sexualität und Sexualerziehung. Mit dem Ziel, die kindliche Sexualität zu befreien und Heranwachsende vor Unterdrückung zu schützen, wird versucht, den Kindern eine Umgebung zu schaffen, in welcher die freie Entfaltung der Sexualität nicht nur möglich, sondern auch gewünscht ist (vgl. Sager 2015: 127f; 131f). Sager bezeichnet diese Entwicklung als „Bruch in der Geschichte der bundesrepublikanischen Sexualaufklärung“ (ebd: 131), welcher nachhaltig Einfluss auf die perspektivische Entwicklung nimmt. Mit den neuen gesellschaftlichen Idealen einher geht eine Anerkennung von kindlicher Sexualität als positiv und entwicklungsfördernd.

Außerdem manifestiert die "neue“ Relevanz des Themas den Bedarf an außerfamiliärer Sexualerziehung, welcher sich im Jahr 1968 die sogenannte Kultusministerkonferenz thematisch annahm (vgl. ebd.: 132f).

\section{Schulische Sexualerziehung von 1968 bis etwa 1990}

Bereits damals liegen bildungspolitische Entscheidungen grundsätzlich in der Entscheidungshoheit der einzelnen Landesregierungen. Um den früheren Erziehungsminister*innen und heutigen Bildungsminister*innen die Möglichkeit zum bildungspolitischen Austausch zu geben, wird im Jahr 1948 die Kultusministerkonferenz durchgeführt, welche sich als Ständige Konferenz der Kultusminister der Länder in der Bundesrepublik Deutschland (KMK) konstituiert (vgl. Sekretariat der Ständigen Konferenz der Kultusminister der Länder in der Bundesrepublik Deutschland o.J. (2)). Sie ist bis dato und war bereits zum damaligen Zeitpunkt ein richtungsweisendes Gremium bestehend aus den zuständigen Minster*innen der Länder und dient zur Koordination von unter anderem Bildungsangelegenheiten, deren Bedeutung länderübergreifend ist. So trägt sie zur Sicherung von Qualitätsstandards in Schulen der gesamten Bundesrepublik bei und erarbeitet Beschlüsse, Empfehlungen und Vereinbarungen zur Orientierung der Landesregierungen. Zusammenfassend stellt die KMK den bildungspolitischen Rahmen, welcher in der Umsetzung innerhalb der jeweiligen Bundesländer detailliert ausgestaltet wird (vgl. Sekretariat der Ständigen Konferenz der Kultusminister der Länder in der Bundesrepublik Deutschland o.J. (1)). Unmittelbar nach der Wiedervereinigung der BRD und der DDR treten auch die Zuständigen der neuen Bundesländer der KMK bei (vgl. Sekretariat der Ständigen Konferenz der Kultusminister der Länder in der Bundesrepublik Deutschland o.J. (2)).

Eine der angesprochenen Rahmenvorgaben verabschiedet die KMK mit den Empfehlungen zur geschlechtlichen Erziehung in der Schule am 03. Oktober 1968. Erstmalig weist ein politisches Organ den Schulen bundesweit eine Mitverantwortung bei der Sexualerziehung von Kindern und Jugendlichen zu (vgl. Hilgers 2004: 9). Ähnlich dem heutigen Verständnis 
Sexueller Bildung (vgl. Kapitel 3.2) verorteten die damaligen Verantwortlichen die Inhalte der Sexualerziehung nicht in einem spezifischen Unterrichtsfach. Vielmehr formulieren sie, dass derartige Erziehungsinhalte im gesamten schulischen Altag pädagogisch verankert sein sollen und keinem konkreten Schulfach zugeordnet werden können. Die Nichteingrenzung der Zuständigkeiten führt dazu, dass grundsätzlich alle Lehrkräfte in die Verantwortung genommen werden, sich an der Sexualerziehung zu beteiligen (vgl. Sager 2015: 132ff). Mit einer Erklärung des Bundesverfassungsgerichtes (1977) knapp zehn Jahre später erfolgt dann die bis dahin fehlende gesetzliche Festlegung der von der $K M K$ ausgesprochenen Empfehlungen und die Festschreibung von Sexualerziehung als Gesamterziehungsaufgabe für Schule und Erziehungsberechtigte. Gestützt von einer Entscheidung des Bundesverwaltungsgerichtes soll die (schulische, aber auch elterliche) Sexualaufklärung fortan weltoffen sein und Diversität aufgrund von verschiedenen Weltanschauungen berücksichtigen (vgl. Hilgers 2004: 9f). Es folgen bundesweite Initiativen zur gesetzlichen Verankerung, aber auch zur Integration der Themengebiete in die jeweiligen Schulgesetze und damit schließlich in die Lehrpläne (vgl. ebd.). Hilgers hält ihrer in ihrer Expertise (beauftragt von der $B Z g A$ ) fest, dass inzwischen „Sexualerziehung in allen Bundesländern als Teil des Erziehungsauftrages der Schule formuliert“ ist (Hilgers 2004: 11).

\section{Schulische Sexualerziehung von 1990 bis in die frühen 2000er Jahre}

Eine zusätzliche Stärkung ihrer Bedeutung erhalten diese Entwicklungen durch eine Erweiterung des Schwangeren- und Familienhilfegesetzes (SFHG) Anfang der 90er Jahre, welche die Sexualaufklärung zu einer bundeslandübergreifenden öffentlichen Aufgabe erklärte (vgl. Hilgers 2004: 11). Die Kommentierung dieses Gesetzes veranlasst mehrere Landesregierungen dazu, ihre Richtlinien und Lehrpläne erneut zu überarbeiten. Sie betont noch einmal nachdrücklich, dass Sexualaufklärung, und damit auch die Sexualerziehung an Schulen, über die reine Vermittlung von Faktenwissen und Informationen hinausgehen muss. Zusätzlich soll sie auch auf emotionaler Ebene wirken und eine Auseinandersetzung mit beziehungsrelevanten Aspekten, unterschiedlichen Lebensstilen und gesellschaftlichen Werten anleiten. Die vom zuständigen Bundesverfassungsgericht als notwendig eingeschätzten qualifizierten Multiplikator*innen sollen als Expert*innen diesen Prozess begleiten und Kommunikation mit den verschiedenen Ziel- und Altersgruppen anregen (vgl. ebd.: 11ff).

Ein bedeutsamer Einschnitt hinsichtlich der schulischen Sexualaufklärung ist im Jahr 2002 zu verzeichnen: Die Empfehlungen der KMK aus dem Jahr 1968 werden aufgehoben, eine Aktualisierung der Empfehlungen seitens der KMK als nicht erforderlich eingeschätzt. Seitdem obliegt die Erarbeitung von Richtlinien und Lehrplänen zur schulischen Sexualaufklärung ausschließlich den Bildungsministerien der einzelnen Bundesländer und orientiert sich nicht 
mehr an den ursprünglichen Empfehlungen der KMK. Aus der detaillierten Analyse Hilgers‘ geht hervor, dass die Inhalte der einzelnen Lehrpläne inzwischen deutlich über die damaligen Empfehlungen der KMK hinausreichen (vgl. ebd. 7). Lehrkräfte aller Schulformen versuchen auf Basis der Rahmenlehrpläne dem schulischen Erziehungsauftrag zur Sexualerziehung nachzukommen. Als ganz zentrale Ziele haben die Bundesländer dabei miteinander übereinstimmend formuliert, dass Kinder bis zum Ende des ersten Schuljahres die unterschiedlichen Geschlechter kennenlernen und über Aspekte von Fortpflanzung (Mutterschaft) aufgeklärt werden sollen. Bis vor Beginn des siebten Schuljahres sollen alle Heranwachsenden außerdem über Themen wie Zeugung und Schwangerschaft, aber auch Kenntnisse zu anderen biologischen Prozessen wie der Pubertät oder sexuell übertragbare Krankheiten erworben haben. Damit verbunden ist eine erste Auseinandersetzung mit den Themenfeldern sexualisierte Gewalt und sexueller Missbrauch an Kindern (vgl. Hilgers 2004: 15f).

Hilgers' Expertise weist nach, dass bis in die frühen 2000er Jahre ebenfalls eine bundesweite Vereinheitlichung der Lehrpläne dahingehend erfolgt, dass gesellschaftlich bewährte Lebensräume wie Ehe und Familie als bevorzugte Lebensräume für Sexualität vermittelt werden. Diese Lebensräume entsprechen in ihrer Gestaltung dabei einem sehr traditionellen Verständnis und beschränken sich überwiegend auf gemischtgeschlechtliche Konstellationen (vgl. Hilgers 2004: 166ff). Über einen langen Zeitraum bewegen sich die in den Schulen vermittelten Positionen in einem Aushandlungsfeld zwischen der Aufklärung zu medizinischen Fakten, der Warnung vor Risiken und Informationen zu Familienplanung und einem eher auf emotionaler und sozialer Ebene ansetzenden Bestreben von sexualitätsbezogenen Betätigungen außerhalb von Fortpflanzung und Ehe (vgl. Milhoffer 2013: 582ff). Ebenso gibt es Untersuchungen, die nachweisen, dass schulische Lehrmaterialien bis vor wenigen Jahren überwiegend traditionelle Rollenbilder auf Basis einer zweigeschlechtlichen Heteronormativität propagieren und Diversität hinsichtlich Geschlecht und Lebensform, wenn überhaupt, dann zumeist problembehaftet thematisch eingeführt wird (vgl. Bitter 2011: 74ff).

\section{Schulische Sexualerziehung der Gegenwart}

Seit einigen Jahren sind nun allerdings Veränderungen in der schulischen Sexualerziehung zu verzeichnen. Ausgehend davon, dass Gesellschaft einem steten Wandel unterliegt, werden in den vergangenen zwei Jahrzehnten vielfach Prozesse angestoßen, die zu einer gesellschaftlichen Auseinandersetzung mit Sexualität und vielfältigen Lebensformen sorgen. Traditionelle Rollenbilder und geschlechtliche und sexuelle Identitätszuschreibungen haben sich dabei ebenso lange gehalten wie das Verschließen vor der Realität, dass Menschen selbstverständlich schon immer auch von heteronormativen Geschlechtermodellen abweichende Identitäten entwickelten. Inzwischen befinden wir uns in einer Gegenwart, die 
sich mit der Vielfältigkeit von Menschen, ihrer diversen Sexualität und ihren Identitäten auseinandersetzt und Bestrebungen voranbringt, alle Lebensformen zu integrieren, akzeptieren und zu tolerieren. Nach wie vor kommt es dabei zu Diskriminierung, Ausgrenzung und Ungleichbehandlung von Minderheiten und einer Unterteilung in das Normale und das Andere, was vielfach aufgearbeitet werden muss, um in naher Zukunft überwunden zu werden. Dennoch ist insgesamt ein gesellschaftlicher Prozess zu verzeichnen, der sexuelle und geschlechtliche Vielfalt in den Blick nimmt und nicht mehr kategorisch ausschließt (vgl. Milhoffer 2013: 582). Diese Entwicklung nimmt auch Einfluss auf den Lebensraum Schule und findet beispielsweise in diversen Initiativen Ausdruck. Vorangetrieben werden diese Bestrebungen vielfach von Akteur*innen im Feld der Sexuellen Bildung, welche einen großen Beitrag dazu leisteten, eine emanzipatorische Sexualpädagogik zu etablieren. Milhoffer führt dazu aus, dass diese „zunehmend als wesentlicher Bestandteil einer persönlichkeitsstärkenden Sozialerziehung aufgefasst wird“ (Milhoffer 2013: 583). Aufklärungsarbeit setzt dabei an einem Punkt an, an dem Sexualität als positiver Bestandteil der Lebenswelt von Kindern, Jugendlichen und Erwachsenen betrachtet wird, den es sich in all seinen Facetten zu erschließen gilt: hinsichtlich der eigenen (sexuellen) Identität, der damit verbundenen Verantwortlichkeit gegenüber dem eigenen Körper aber auch gegenüber anderen Menschen, gleichzeitig jedoch ebenfalls hinsichtlich lustvollen sexuellen Begehrens und Betätigens. Ebenso fokussieren schulische Konzepte darauf, das Selbstbewusstsein der Heranwachsenden zu stärken und sie darin zu unterstützen, (auch sexuell) selbstbestimmt eigene Haltungen und Perspektiven zu entwickeln und einzunehmen (vgl. ebd.: 583f).

Weiterhin gibt es in einigen Bundesländern landesweite Initiativen, welche sexuelle Vielfalt in das Handlungsfeld Schule zu integrieren versuchen und hier nur exemplarisch erwähnt werden sollen. So engagiert sich beispielsweise das Antidiskriminierungsprojekt „Schule der Vielfalt - Schule ohne Homophobie" in Nordrhein-Westfalen und regt Schulen dazu an, eine Selbsterklärung zu unterzeichnen und sich gegen Diskriminierung aufgrund geschlechtlicher Identität und/oder sexueller Orientierung einzusetzen. Im Rahmen des Unterrichts werden Themen sexueller und geschlechtlicher Vielfalt thematisiert und tabuisierte Themen wie Homosexualität oder Transsexualität aufgegriffen. Die Initiative zählt inzwischen 26 Projektschulen (vgl. Pohl o.J.).

Viel diskutiert wurde Baden-Württembergs Entscheidung, das Thema sexuelle Vielfalt im Bildungsplan zu verankern, welche ebenfalls vorsieht, im schulischen Kontext aktiv die Auseinandersetzung mit vielfältigen Lebens-, Identitäts-, und Geschlechterformen umzusetzen. Unter anderem wird dabei der traditionelle Familienbegriff verhandelt und die Pluralität von Familienkonstellationen thematisiert. Auch die Berliner Bildungspolitik sieht bereits seit 2001 vor, dass Schulen für Kinder und Jugendliche Klimata schaffen, die ihre sexuelle Entwicklung auch hinsichtlich der Vielfalt sexueller Möglichkeiten fördern. 
Unabhängig von Prozessen, die mitunter auf bildungspolitischer Ebene vorangebracht und unterstützt werden, ist die Ausbildungssituation von Lehrkräften hinsichtlich Sexueller Bildung, wie aufgezeigt, sehr defizitär. Gleichermaßen sind bis heute noch keine Qualitätsstandards für Inhalte von Fort- und Weiterbildung aktiver Lehrkräfte auf dem Themengebiet verabschiedet (vgl. ebd.: 584). Die tatsächliche Situation im schulischen Alltag ist demzufolge im höchsten Maß von dem schulischen Konzept, seiner Umsetzung und der persönlichen Konstitution der Lehrkraft (hinsichtlich Methodik und Didaktik) sowie ihrer eigenen Wertevorstellungen abhängig.

\subsection{Lehrer*innen als Personen der Sexualaufklärung}

Neben zahlreichen weiteren Projekten, Kampagnen, Initiativen und in Auftrag gegebenen Expertisen veranlasste die $B Z g A$ im Jahr 2015 eine Repräsentativbefragung zur Jugendsexualität. Die inzwischen achte Studie der Reihe fokussiert auf die Perspektive von 14 - 25-jährigen jungen Menschen und bildet die Entwicklung zu Einstellungen und Verhalten der befragten Personen im Kontext von Sexualität seit 1980 ab. Wie Abbildung 4 zu entnehmen, verdeutlichen die Befragungsergebnisse, dass auch die Jugendlichen selbst Lehrkräfte als - nach den Eltern und z.T. den engsten Freund*innen - wichtige Personen hinsichtlich ihrer sexuellen Aufklärung wahrnehmen (vgl. BZgA Bode / Heßling 2015: 14).

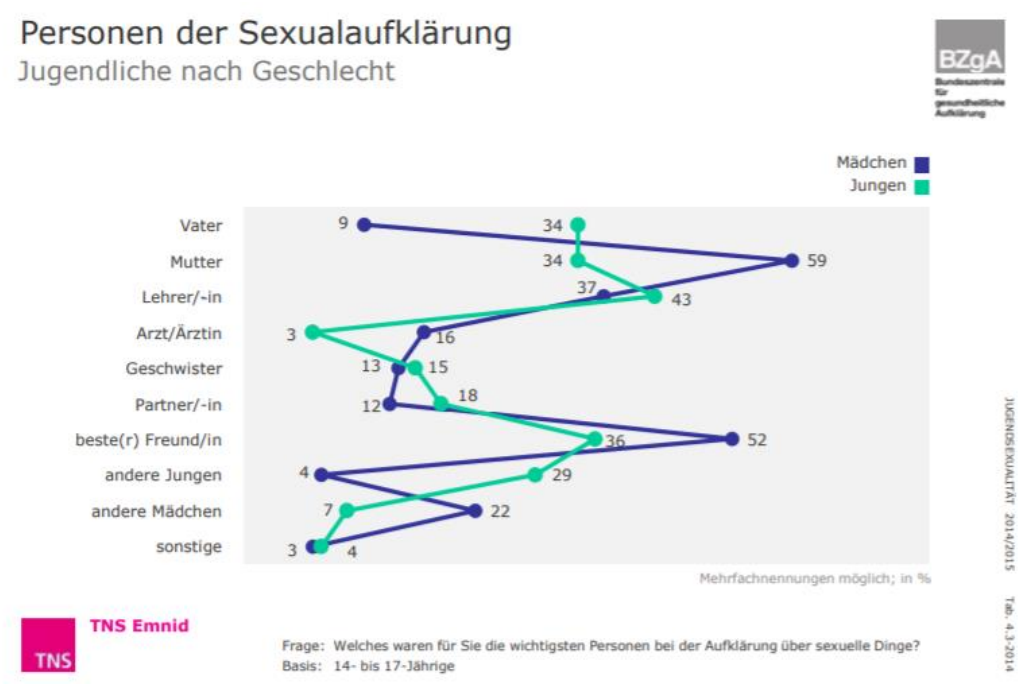

Abb. 2: Personen der Sexualaufklärung. Jugendliche nach Geschlecht

Quelle: Bode/Heßling 2015: 14.

Abbildung 4 zeigt ebenfalls, dass sich die Ausprägung der Relevanz von Lehrkräften hinsichtlich der Sexualaufklärung nach Geschlecht und Herkunft unterscheidet. In allen Fällen wird Lehrer*innen jedoch eine durchaus bedeutsame Rolle seitens der Jugendlichen zugesprochen. 


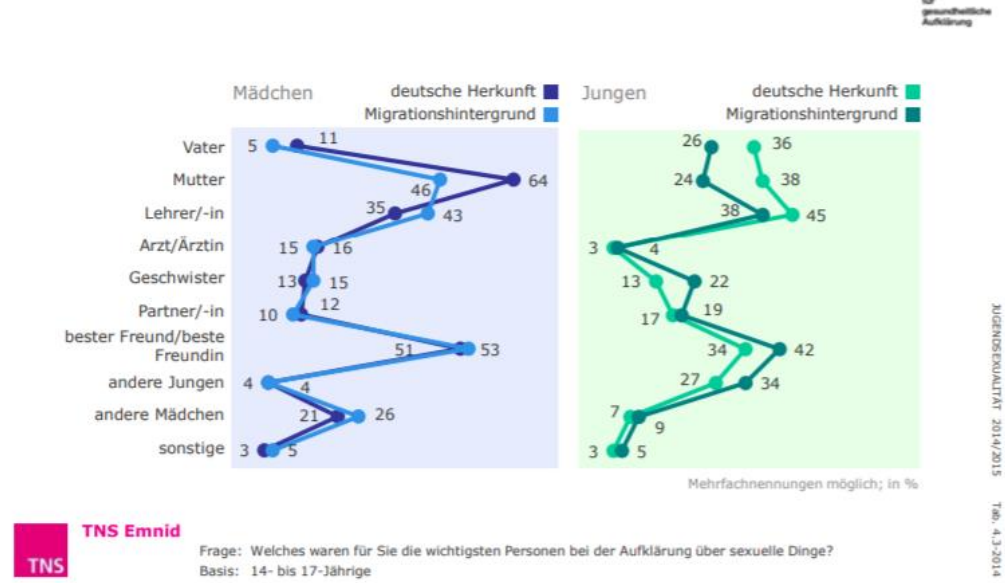

Abb. 5: Personen der Sexualaufklärung. Jugendliche nach Herkunft

Quelle: Bode/Heßling 2015: 19.

Je nach Geschlechts- und Herkunftszuordnung nehmen Lehrkräfte dabei mitunter eine bedeutendere Rolle als in früheren Befragungen ein, was abermals die Relevanz der Sexuellen Sozialisation im schulischen Kontext untermauert. So benennen beispielsweise Jungen (unabhängig ihrer Herkunft) Lehrer*innen erstmalig noch vor ihren Müttern als Hauptakteure der Sexualaufklärung, was sowohl aus Abbildung 4 als auch in Abbildung 5 ersichtlich wird.

Hinsichtlich der in Schulen tatsächlich stattfindenden Sexualaufklärung hat es in den vergangenen Jahren eine deutliche Entwicklung gegeben (vgl. Abbildung 6).
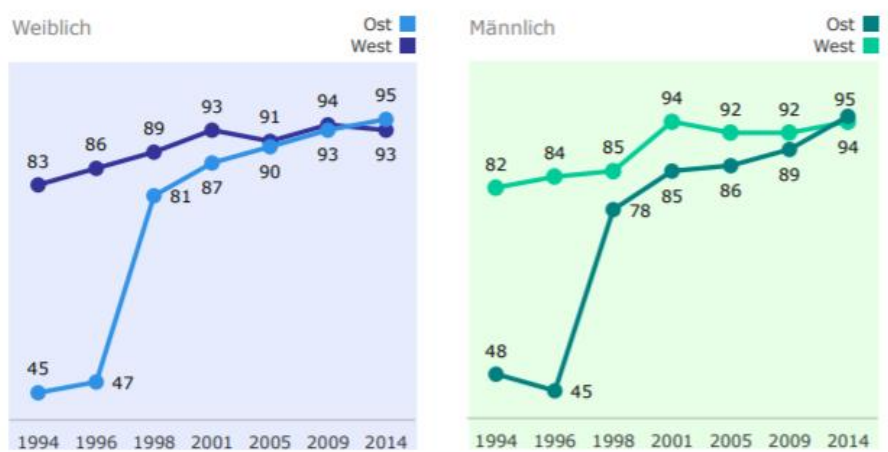

1994199619982001200520092014

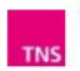

TNS Emnid

$$
\begin{aligned}
& \text { Frage: Haben Sie Sexualkunde- bzw. Sexualerziehungsthemen im Unterricht } \\
& \text { Basis: 14- bis 17-jahrige deutscher Staatsangehörigkeit/ab 2014: Herkunft }
\end{aligned}
$$

Abb. 6: Sexualaufklärung in der Schule. Langzeittrend deutsche Jugendliche nach Region

Quelle: Bode/Heßling 2015: 34. 
Mehr als neun von zehn Jugendlichen geben an, sich in der Schule mit Themen zu Sexualität beschäftigt zu haben. Inzwischen gibt es dahingehend auch kaum noch nennenswerte Unterschiede zwischen den alten und neuen Bundesländern der Republik.

Die Ergebnisse der repräsentativen Befragung zeigen deutlich, dass Sexuelle Bildung, zumindest jedoch Vermittlung konkreten Wissens, unbestritten relevant für den Lebensraum Schule ist.

Daran knüpfen auch die Ergebnisse des Forschungsprojekts „Teilhabechancen an sexueller Bildung von Jugendlichen und jungen Erwachsenen mit kognitiver Beeinträchtigung in Sachsen" an (vgl. Kapitel 3.3; vgl. Wienholz/Retznik 2018 (2): 2). Auch in der hier durchgeführten Untersuchung geben Jugendliche $(n=42)$ Lehrer*innen als die Personen an, durch die die Sexualaufklärung am Häufigsten stattfindet. Dies wird durch die Antworten der befragten Lehrkräfte $(n=100)$ unmittelbar verifiziert, die sich selbst nicht nur eine bedeutsame Rolle hinsichtlich sexueller Fragen von Heranwachsenden zuweisen. Insgesamt 66\% stellen darüber hinaus einen Mangel an adäquater Sexualaufklärung der jungen Menschen durch das Elternhaus fest und identifizieren damit sich selbst in ihrer Rolle als Lehrer*innen in der Verantwortung, diesen Mangel zu kompensieren (vgl. Wienholz/Retznik 2018 (2): 2ff). Nichtsdestotrotz verweisen die befragten Personen in der Frage nach der Hauptverantwortlichkeit - neben sich selbst - auch auf die Eltern, wie Abbildung 7 verdeutlicht:

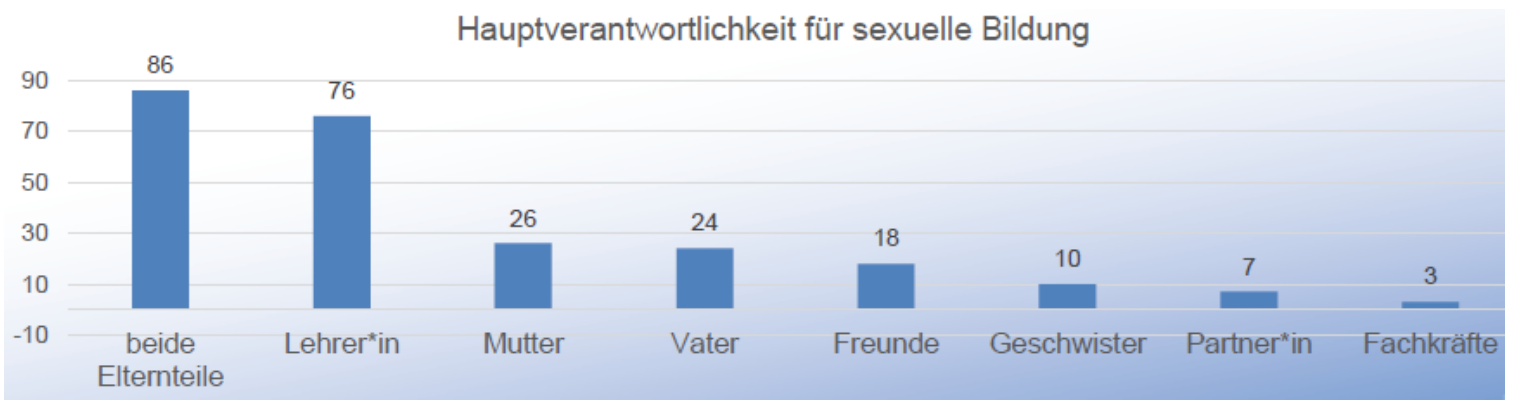

Abb. 7: Hauptverantwortlichkeit für sexuelle Bildung

Quelle: Wienholz/Retznik 2018 (2): 5.

Die hier dargestellten Angaben beziehen sich lediglich auf die Begrifflichkeit Sexualaufklärung. Wie in den Kapiteln 1.2 und 3.3 erläutert, stellt Sexuelle Bildung zusätzliche Ansprüche an Lehrkräfte beziehungsweise zuständiges Bildungspersonal. Dass Lehrkräften in der sexuellen Sozialisation von Heranwachsenden eine bedeutsame Aufgabe zukommt, ist bereits mit den erläuterten Untersuchungen unbedingt anzunehmen. Inwiefern tatsächlich eine Auseinandersetzung mit Inhalten Sexueller Bildung über die reine Wissensvermittlung hinaus erfolgt und inwieweit Lehrkräfte im Studium entsprechende Grundlagen erlernen, soll im Folgenden erläutert werden. 


\subsection{Konzept der Sexuellen Bildung}

Um ein umfassendes Bild davon zu zeichnen, inwieweit Inhalte Sexueller Bildung in die schulische Sexualerziehung beziehungsweise Sexualaufklärung einfließen, ist es notwendig, das Konzept der Sexuellen Bildung dezidiert zu erläutern.

Sexuelle Bildung nach heutigem sexualwissenschaftlichem Verständnis ist das Ergebnis einer relativ jungen Entwicklung. Valtl beschreibt Sexuelle Bildung als die aktuelle Ausprägung von Sexualpädagogik und identifiziert fünf zentrale Kennzeichen des Konzepts Sexueller Bildung (vgl. Valtl 2013: 128ff). Er weist darauf hin, dass das Konzept selbst keineswegs neu ist, bezeichnet die Sexuelle Bildung aber als „neues Paradigma einer Sexualpädagogik für alle Lebensalter" (ebd.: 125) und führt damit eine Verschiebung der Akzente, die sich über mehr als 50 Jahre bis heute vollzieht, in einem neuen Begriff zusammen. Als erstes konkretes Merkmal des Konzepts hält er fest:

(Sexuelle) Bildung ist „selbstbestimmt und lernzentriert“ (ebd: 128).

Dies resultiert insbesondere daraus, dass Sexuelle Bildung einen gesellschaftlichen Kontext gefunden hat, der sich deutlich von Erziehung, also einer einseitig bestimmten Handlung, abgrenzt (vgl. ebd.). Im Gegensatz zur Erziehung hat der sich bildende Mensch die Möglichkeit, unter Begleitung von Erziehenden sich die Lerninhalte interaktiv anzueignen (vgl. ebd.). Diese Ansicht birgt immanent eine Akzeptanz dafür, dass Menschen jeden Lebensalters sich ihrer sexuellen Identität durch eigene Erfahrungen, Ausprobieren des (Un-)Bekannten und dem Reflektieren des Erlebten nähern dürfen und sollen. Gleichzeitig bezieht sich die Selbstbestimmung auch darauf, dass es in der Entscheidung der jeweiligen Zielgruppe von Sexueller Bildung liegt, inwieweit sie sich auf die Angebote Sexueller Bildung einlassen und welcher Inhalte sie sich letztlich annehmen wollen (ebd.: 129). Valtl konstatiert, dass Lernende die gleichen Schlüsselqualifikationen, die sie auch in der Auseinandersetzung mit anderen Lerninhalten nutzen, auf Sexualität anwenden könnten. Folgerichtig ist es deshalb in erster Linie Aufgabe der Sexuellen Bildung, Kompetenzen wie Problemlösungsstrategie oder Wissen über Möglichkeiten der Informationsbeschaffung im Hinblick auf Sexualität zu vermitteln. Dadurch werden Lernende darin befähigt, sich sinnvoll mit sexuellen Fragen auseinanderzusetzen und ihre eigenen Antworten auf diese zu finden, um zu ihrer individuellen sexuellen Identität zu gelangen (vgl. ebd.: 129). Die Aufgabe für die Akteur*innen im Feld der Sexuellen Bildung besteht in erster Linie darin, Angebote zu unterbreiten, die passend zur Zielgruppe sind und ihren Bedarfen entsprechen. Nur auf diesem Wege ist es möglich, dass Grundprinzip der Sexuellen Bildung umzusetzen, dass Adressat*innen selbst zur Umsetzung der Angebote und ihrem Ertrag (für sie selbst) beitragen (vgl. ebd.). Valtls Hinweis, dass auch die Lernumgebungen so aufbereitet sein müssen, dass sie für die Zielgruppen nutzbar sind, ist ebenso wichtig wie selbsterklärend: Sollen beispielsweise Kinder und Jugendliche selbst in Aktion treten und sich mit Themen wie Sexualität, Diversität und geschlechtlicher Identität 
auseinandersetzen, muss die Umgebung geeignete Voraussetzungen dafür bereitstellen. Dies meint insbesondere, dass anleitende Personen Lernräume vorhalten, die in einem möglichst freien Rahmen Lernen ermöglicht - und damit nicht zuletzt frei von Diskriminierung und Ausgrenzung sind (vgl. Valtl 2013: 129). Im weiteren Verlauf knüpft Valtl eng an Prinzipien an, mit denen die Soziale Arbeit bereits seit vielen Jahren vertraut ist. Die Adressat*innen sind die Expert*innen für sich selbst und sollen eigenmächtige Entscheidungen treffen und treffen dürfen. Es ist nicht die Aufgabe von Akteur*innen der Sexuellen Bildung, den Teilnehmenden von Angeboten Entscheidungen abzunehmen oder sie ihnen aufzudrängen. Vielmehr sind sie in der Verantwortung, die Voraussetzungen dafür zu schaffen, dass die Adressat*innengruppe selbstbestimmt zu handeln lernt. Es handelt sich bei diesem Vorgehen um eine Art Vertrauensvorschuss, der davon ausgeht, dass Menschen in der Lage sind Fähigkeiten zu nutzen, die sie gerade erst entwickeln. Dieses Prinzip und die Notwendigkeit auf Seiten der Fachkräfte, Entscheidungen auszuhalten und zu akzeptieren, die sie in dieser Form möglicherweise nicht selbst treffen würden, ist gleichzeitig eine der größten Herausforderungen des ersten Merkmals Sexueller Bildung (vgl. ebd: 129f).

Als zweiten wesentlichen Punkt erläutert Valtl:

\section{„Sexuelle Bildung hat einen Wert an sich." (ebd.: 131)}

Mit der Annahme, dass Sexuelle Bildung nicht mehr nur eingesetzt wird, um ein bestimmtes Ziel zu erreichen, sondern bereits für sich stehend Wert hat, werden frühere Ideen von Sexualpädagogik oder Sexualerziehung nicht verworfen. Im Gegenteil integriert Sexuelle Bildung vormals identifizierte Aufgabengebiete wie Wissensvermittlung und Zielgruppen, beispielsweise auch bildungsferne Kinder und Jugendliche (vgl. ebd.). Über diese Grundbildung hinaus versteht sich Sexuelle Bildung jedoch auch als Botschafterin von Sexualität, ihrer Bedeutung und ihren zentralen Momenten wie Sinnlichkeit, Lustempfinden und Liebesfähigkeit. Sielert bezeichnet dies als „Sensibilisierung der Sinne und Sinnlichkeit (...) [, welche] die Reflexion und Kultivierung von Körperlichkeit, der sinnlichen Ausstrahlung, der Wechselwirkung von Selbst- und Fremdwahrnehmung, d[ie] Balance von Selbstwertgefühl, Ich-Ideal und äußerer Erscheinung sowie die aktive Gestaltung der Selbstpräsentation“ einschließt (Sielert 2015 (1): 27).

Folgt man Valtls Ausführungen, gelangt man zum dritten Kennzeichen Sexueller Bildung: „Sexuelle Bildung ist konkret und brauchbar" (Valtl 2013: 133)

Mit dieser Aussage untermauert Valtl das Bestreben, dass Sexuelle Bildung zieloffen sein muss und nicht abhängig von den Werte- und Normvorstellung der ausführenden Personen sein darf (vgl. ebd.). Ein zentraler Baustein ist es, den Nutzer*innen von Sexuellen Bildungsangeboten ein konkretes, also authentisches Bild der Realität zu vermitteln. Losgelöst von persönlicher Bewertung können so beispielsweise Kinder und Jugendliche selbst zur moralischen Reflexion mit gesellschaftlich relevanten sexuellen Elementen gelangen (vgl. 
ebd.). Um diesen Schritt vollziehen zu können, bedarf es einer Konfrontation mit sexuellen Kulturgütern, die sich bis dato als brauchbar hinsichtlich der sexuellen Kulturentwicklung erwiesen haben. Dies können Dinge wie Verhütungsmittel, Erotik in Filmen oder Fotografien, aber auch gesetzliche Grundlagen sein. In diesem Kontext birgt die Gruppe der Adressat*innen gleichzeitig auch die Chance, durch eigenes Wirken und dem Enttabuisieren von Sexualität und den sie umgebenden Aspekten neue sexuelle Kulturgüter hervorzubringen. Insbesondere die gesellschaftlichen Entwicklungen der Gegenwart machen dies zum einem nötig, zum anderen möglich. Einflüsse aus anderen Kulturen können als Chance und Herausforderung zugleich verstanden werden, sind aber in jedem Fall ein möglicher Ansatzpunkt für gesellschaftliche Diskurse. Dabei hilfreich könnte es beispielsweise sein, die Kreativität und die Problemlösungsstrategien von Kindern und Jugendlichen anzuerkennen und zu nutzen, welche sich oftmals als sehr wirksam erweisen. Auch Ableitungen aus therapeutischen Ansätzen sind denkbar und könnten bei der Aufarbeitung dieser Aufgabe wertvollen Input leisten (vgl. ebd. 134ff).

In Übereinstimmung mit Valtl formuliert Sielert ein viertes Merkmal:

„Sexuelle Bildung spricht den ganzen Menschen an, also alle Lebensalter und zwar kognitiv, emotional, sozial und spirituell“ (Sielert 2015 (2): 98).

Es ist die Aufgabe Sexueller Bildung, Menschen in allen Lebenslagen, aber auch allen Altersgruppen diejenigen Kompetenzen zu vermitteln, die sie benötigen, um (sexuell) selbstbestimmt zu leben. Die Inhalte der Angebote sind folgerichtig nicht loslösbar von den individuellen Bedarfen der jeweils adressierten Person und unterscheiden sich daher in höchstem Maß. Es handelt sich dabei um alle denkbaren Aspekte, die Menschen dazu befähigen, sich selbst (sexuell) zu ermächtigen. Dabei werden Kompetenzen wie beispielsweise die Fähigkeit, sich abgrenzen zu können, angesprochen, aber auch das Erlernen der vielfältigen Funktionen und Ausdrucksformen von Sexualität als mögliches Thema identifiziert. Nicht zuletzt geht es darum, Menschen vor sexualisierten Übergriffen zu schützen und dahingehend präventiv zu wirken, indem es Akteur*innen gelingt, im Austausch mit den Zielgruppen eine Sprache für sexuelle Themen zu vermitteln, was die Grundvoraussetzung für präventives, aber auch jedes andere Wirken von Sexueller Bildung darstellt (vgl. Sielert 2015 (1): 26; vgl. Sielert 2015 (2): 98).

Als fünften und letzten Punkt beschreiben Sielert und Valtl deckungsgleich Sexuelle Bildung wie folgt:

„Sexuelle Bildung ist politisch“ (Sielert 2015 (2): 98; Valtl 2013: 137)

Sowohl als einwirkende Größe als auch als Empfängerin ist Sexualität politisch wirksam beziehungsweise adressierbar. Daraus ergibt sich zwangsläufig auch eine Politik der Sexuellen Bildung, deren Aufgabe sich daraus ableitend darin wiederfindet, ein Bewusstsein für diese Zusammenhänge zu schaffen. Es gilt insbesondere politische Themen wie 
gesellschaftliche Herrschafts- und Machtverhältnisse und daraus entstehender sexualisierter Gewalt sowie Diskriminierung von Diversität und Vielfalt aufzubereiten, um Nutzer*innen Sexueller Bildung zu befähigen, am gesellschaftlichen Diskurs teilzunehmen und aktiv für ihre Überzeugungen eintreten zu können (vgl. Sielert 2015 (2): 98).

\subsection{Qualifizierungsstand von Lehrkräften mit Fokus auf Studium und Fortbildung} Im wissenschaftlichen Diskurs gibt es bisher nur punktuelle Erhebungen, die Einblick dahingehend gewähren, inwieweit Lehramtsstudierenden Angebote mit Inhalten Sexueller Bildung vorgehalten werden und inwiefern sie und bereits im Schuldienst tätige Lehrkräfte sich kompetent darin fühlen, Aspekte sexueller Bildung im Schulalltag zu vermitteln. In seiner Expertise zur Sexualerziehung an Grundschulen untersuchte Sielert 2011 unter anderem, inwiefern Personen im Referendariat zur Sexualerziehung qualifiziert sind und sich als qualifiziert empfinden. Er erhob außerdem auch Aussagen von Berufspraktiker*innen und fokussierte auf die Situation in Schleswig-Holstein, Hamburg und Bremen. In der Befragung erkennen zunächst 93\% der befragten Personen ( $n=93$, Lehrkräfte an Grundschulen) eine Notwendigkeit darin, dass Lehrer*innen auf die Sexualerziehung vorbereitet sein müssen. Trotz dessen, dass Praktiker*innen die Wichtigkeit der Vorbereitung und damit eine adäquate Qualifizierung hervorheben und es naheliegt, diese Vorbereitung in Form von Ausbildung im Studium zu verorten, scheint die Lehramtsausbildung ihrer dahingehenden Aufgabe nicht nachzukommen. So geben mehr als drei Viertel der Befragten an, im Studium nicht für die Sexualerziehung ausgebildet worden zu sein (vgl. Sielert 2011: 50ff). Lediglich $11 \%$ empfanden die Ausbildung hinsichtlich der Thematik Sexualerziehung im Studium als sehr gut oder gut. Die Ergebnisse Sielerts decken sich mit denen Altenburgs, die 2016 87\% der an ihrer Befragung teilgenommenen Hochschulen identifizierte, nur in (zu) geringem Maß Angebote zum Thema Sexualität vorzuhalten. Zusätzliche $3 \%$ der Hochschulen gaben derartige Angebote als nicht vorhanden an (vgl. Altenburg 2016: 79ff). 

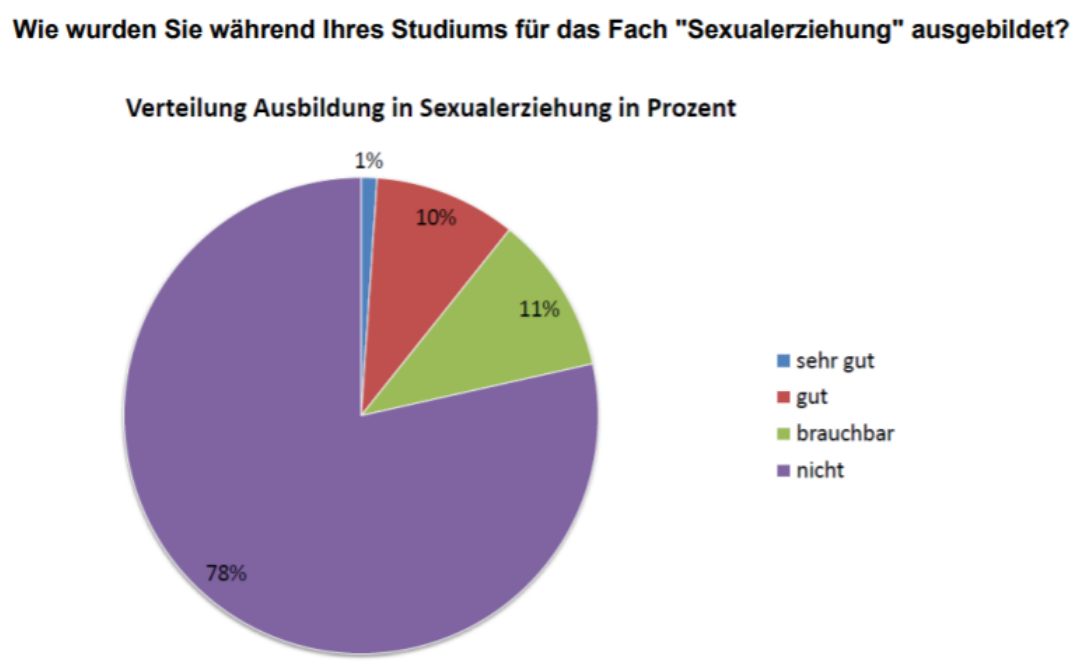

Abb. 8: Verteilung Ausbildung in Sexualerziehung in Prozent

Quelle: Sielert 2011: 50.

Hopf benennt konkrete Missstände und begründet die mangelnde Ausbildung der Lehrer*innen darin, dass es beispielsweise nicht ausreichend Hochschullehrer*innenstellen gibt, um den Bedarf an Inhalten Sexueller Bildung im Lehramtsstudium zu decken. Dadurch, dass Angebote nur punktuell vorgehalten werden können, kann die angestrebte fächerübergreifende Qualifizierung der Lehrkräfte kaum gelingen (vgl. Hopf 2013: 782). Inwiefern damit eine ganzheitliche Sexualaufklärung, wie sie Rahmenlehrpläne vorsehen und auch von der $B Z g A$ und der Weltgesundheitsorganisation (WHO) gefordert und gefördert werden, zum gegenwärtigen Zeitpunkt realistisch ist, ist daher sehr fraglich. Erstaunlich ist, dass aus früheren punktuellen Erhebungen zum Thema Qualifizerung(sbedarfen) von Lehrer*innen hinsichtlich Sexueller Bildung offenbar kaum Ableitungen umgesetzt werden konnten. Auch Erhebungen aus den 80er und 90er Jahren eint insbesondere die Erkenntnis, dass die Mehrheit der Lehramtsstudierenden und späteren Lehrkräfte sich nicht umfänglich darauf vorbereitet fühlt, im Schulalltag professionell mit Themen von Sexualität umgehen zu können. Nach wie vor findet sich nur eine überschaubare Bandbreite von Inhalten, die im Lehramtsstudium verhandelt wird (vgl. ebd.). Zu diesem Ergebnis kommt auch Kollender, die für das Bundesland Nordrhein-Westfalen 2015 ebenfalls konstatiert, dass es kein flächendeckendes Lehrangebot für werdende Lehrkräfte gibt (vgl. Kollender: 100f). Auch ihre Erhebung lässt darauf schließen, dass eine wesentliche Ursache dafür die mangelnde Anzahl an Hochschullehrer*innen ist (vgl. ebd.). Hinzu kommt, dass die Implementierung von Inhalten Sexueller Bildung laut Kollenders Erhebung auch oftmals von der Motivation der Dozierenden und deren persönlichem Interesse am Thema abhängig ist (vgl. Kollender: 100f; vgl. Hopf 2013: 783).

Ergänzend zu den bisher herangezogenen Untersuchungen sei ein Forschungsvorhaben 
erwähnt, welches im Auftrag der $B Z g A$ - neben anderen Schwerpunkten - ebenfalls Erkenntnisse zum Qualifizierungsstand von Lehrkräften im Kontext Sexueller Bildung hervorbringt. Mit Fokus auf Lehrpersonal an Schulen mit sonderpädagogischem Förderbedarf wird im Forschungsprojekt „Teilhabechancen an sexueller Bildung von Jugendlichen und jungen Erwachsenen mit kognitiver Beeinträchtigung in Sachsen” eine Zielgruppe untersucht, die bisher nicht im Spezifischen beforscht wurde. An der durchgeführten Befragung $(n=100)$ nahmen neben Lehrer*innen auch 35 pädagogische Unterrichtshilfen sowie zwei Schulsozialarbeiter*innen teil, von denen ein Großteil (78\%) bereits selbst Sexuelle Bildung vermittelt hat. In Summe geben 37\% des Lehrpersonals an, dass Inhalte Sexueller Bildung bereits während ihrer Ausbildung Bestandteil waren. Die Tatsache, dass die Wahrscheinlichkeit für eine Thematisierung von Sexueller Bildung innerhalb der Ausbildung stieg, je jünger die befragten Personen waren, lässt darauf schließen, dass zumindest in Ansätzen Veränderungen in Ausbildungsinhalten zu verzeichnen sind. Obgleich darüber hinaus fast die Hälfte der Lehrkräfte (44\%) angeben, auf Initiative ihres Arbeitsgebers eine Fort- oder Weiterbildung mit Inhalten Sexueller Bildung besucht zu haben, fühlen sie sich in der Mehrzahl der Themenbereiche nicht umfänglich informiert, was Abbildung 9 veranschaulicht:

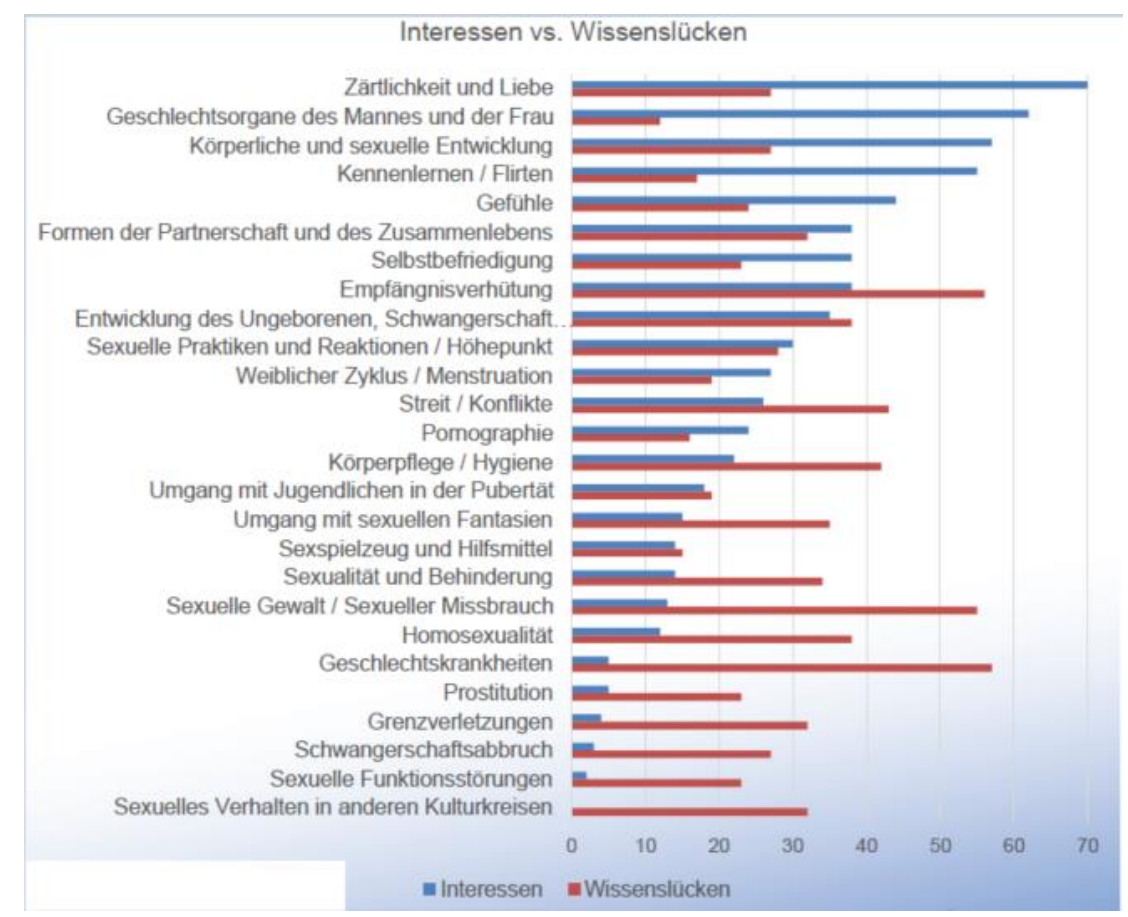

Abb. 9: Interessen vs. Wissenslücken

Quelle: Wienholz/Retznik 2018 (2): 6.

Inwieweit existierende Fort- und Weiterbildungsangebote mit Inhalten Sexueller Bildung den Bedarfen bereits aktiver Lehrkräfte gerecht werden und mitunter fehlende Ausbildungsinhalte kompensieren können, ist bisher nicht wissenschaftlich untersucht worden. 


\subsection{Aktuelle Entwicklungen in der Forschungslandschaft}

Über unsere Landesgrenzen hinaus ist das Recht auf Information und Bildung ein anerkanntes Menschenrecht. So wurde es beispielsweise in der UN-Kinderrechtskonvention im Jahr 1989 festgehalten und noch dahingehend ergänzt, dass Kinder auch das Recht darauf haben, Zugang gewährt zu bekommen „zu Informationen und Material aus einer Vielfalt nationaler und internationaler Quellen, insbesondere derjenigen, welche die Förderung seines sozialen, seelischen und sittlichen Wohlergehens sowie seiner körperlichen und geistigen Gesundheit zum Ziel haben“ (UNICEF o.J.: 21). Die unterzeichnenden Staaten haben sich darüber hinaus dazu verpflichtet, entsprechende Bildungsangebote bereitzustellen und zu fördern (vgl. ebd.). Entsprechend politisch gestärkt haben die $B Z g A$ und das Regionalbüro für Europa der WHO im Jahr 2008 ein Vorhaben initiert, welches „Standards für die Sexualaufklärung in Europa“ etablieren möchte. Die Standards sollen Orientierung dafür geben, in europäischen Ländern ganzheitliche Sexualaufklärung einzuführen und entsprechende Curricula zu entwickeln. Gleichzeitig beabsichtigt die Initiative durch ihre Außenwirkung politisch wirksam zu sein, Dialogmöglichkeiten zu schaffen und langfristig potentielle Kritiker*innen von der Notwendigkeit einer solchen ganzheitlichen Sexualaufklärung überzeugen zu können (vgl. BZgA 2011: 7ff). Wie unter 3.4 erläutert, gibt es großen Nachholbedarf hinsichtlich der Qualifizierung von Lehrkräften im Themenfeld Sexuelle Bildung. Einen weiteren Missstand weist der Runde Tisch auch in seinem Abschlussbericht im Jahr 2011 auf. Im Hinblick auf die Prävention sexualisierter Gewalt identifizieren die Expertinnen einen erheblichen Qualifizierungsbedarf und fordern gleichzeitig explizit eine nachhaltige Implementierung der Aus- und Fortbildung für Lehramtsstudierende sowie für pädagogisch tätiges Personal (vgl. Runder Tisch 2011/2012: 9, 38ff, 191ff). Konkret formuliert der Runde Tisch die Erwartung, „dass in den öffentlichen Schulen sowie in den Schulen in freier Trägerschaft durch geeignete Maßnahmen Prävention und Intervention gestärkt und nachhaltig verbessert werden" (ebd.: 191). Der Runde Tisch erkennt die Institution Schule in seiner Argumentation als Orte an, an denen nahezu alle Kinder und Jugendlichen einen erheblichen Teil ihres Alltages verbringen und damit erreichbar sind. Um Prävention voranzubringen und Heranwachsende sicher vor Übergriffen und frei in ihrer sexuellen Selbstbestimmung aufwachsen zu lassen, definiert der Runde Tisch Schulen als möglichen Schutzraum. In diesem nehmen Lehrer*innen eine besondere Rolle ein, da sie als Bezugspersonen alters- und entwicklungsgerechte Fragen von Sexualität aufgreifen und damit maßgeblich Einfluss auf die sexuelle Sozialisation der Kinder und Jugendlichen nehmen. Sowohl die vermittelten Kompetenzen als auch eine gefestigte, sexuell selbstbestimmte Persönlichkeit, die aus eben diesen Kompetenzen erwachsen soll, können Schüler*innen dabei unterstützen, sich gegen sexualisierte Übergriffe zu schützen (vgl. ebd.: 38f).

Bereits in der ersten Auflage der BMBF-Förderlinie „Forschung zu sexualisierter Gewalt gegen 
Kinder und Jugendliche in pädagogischen Kontexten“ zielten einige der geförderten Forschungsvorhaben auf einen Erkenntnisgewinn zu Haltungen, Herangehensweisen und Bedarfen von Lehrkräften hinsichtlich Sexueller Bildung und der Prävention sexualisierter Gewalt. Auch unter der 2017 erschienen neuen Förderbekanntmachung zum gleichen Forschungsschwerpunkt gibt es abermals Projekte, deren Forschungsziele den Kompetenzgewinn von Lehrkräften bezüglich Themen Sexueller Bildung anstreben, um so langfristig Prävention voranzubringen (vgl. Illichmann 2017).

Auch in anderen Initiativen des BMBFs knüpft die Bundesregierung an den Erkenntnissen des Runden Tisches an und versucht Lehrer*innen in ihren - über die für den reinen Fachunterricht hinaus notwendigen - Kompetenzen zu stärken (vgl. ohne Autor, o. J. (2)). In ebenfalls zwei Förderperioden soll die reformierte Hochschulausbildung der „Experten für das Lehren und Lernen“ (ebd.) unterstützt werden, um den neuen Anforderungen des Berufs gerecht zu werden und Kinder und Jugendliche in nahezu allen Lebensbereichen kompetent begleiten zu können.

Selbstverständlich gibt es noch zahlreiche weitere Bundesinitiativen und Projekte, die aus beispielsweise Hochschuletats oder sonstigen Budgets finanziert werden. Leider fehlt im deutschsprachigen Raum eine gebündelte Gesamtübersicht über derartige Vorhaben. Zur Zusammenführung der jeweiligen Ergebnisse und einer flächendeckenden Verstetigung derselben, wäre diese jedoch sehr hilfreich. Eine intensive Betrachtung der einzelnen Aktivitäten würde jedoch nicht den Schwerpunkten der hier vorliegenden Untersuchung entsprechen und sollte deshalb an anderer Stelle aufgegriffen werden. 


\section{Schule und sexualisierte Gewalt - Theoretischer Kenntnisstand}

Die Thematik Schule und sexualisierte Gewalt ist eine differenziert zu betrachtende Verbindung. Sie zielt auf verschiedenste Dimensionen, denen in einer intensiven Betrachtung versucht werden muss, Rechnung zu tragen.

Zunächst sollte hinterfragt werden, inwieweit sich die Schulen innerhalb eines institutionell vorgegebenen Rahmens mit sexualisierter Gewalt auseinandersetzt. Zu dieser Auseinandersetzung könnten aufklärende Projekte, Unterrichtsgegenstände bezüglich der Thematik sexualisierter Gewalt oder präventive Angebote zählen. Diese Aspekte sind Bestandteil Sexueller Bildung und mitunter auch schulischer Sexualerziehung. Wie bereits erläutert, sind die tatsächlichen Inhalte schulischer Sexualerziehung eng verknüpft mit der Frage danach, wie kompetent Lehrkräfte darin sind, diese zu begleiten, durchzuführen oder zu initiieren. Dies wiederum steht untrennbar damit in Zusammenhang, inwieweit es Lehrkräfte als ihre eigene Aufgabe ansehen, derartige Angebote vorzuhalten oder zu ermöglichen. Einen entsprechenden Gesamtüberblick gab eine generelle Betrachtung von Sexueller Bildung im Kontext von Schule, welche sowohl geltende Rahmenrichtlinien als auch die Inhalte in der Lehramtsausbildung einschloss (vgl. Kapitel 3; vgl. Kapitel 3.3).

Über thematische Aushandlungen in Unterrichts- oder unterrichtsähnlichen Situationen hinaus muss man Schule auch als Ort in den Blick nehmen, an dem sexualisierte Gewalt ausgeübt werden kann und ausgeübt wird. Entgegen dem angestrebten Schutzraum werden schulische Bildungseinrichtungen dabei zu Gelegenheiten, in denen sowohl Erwachsene als auch Heranwachsende sexuell übergriffig sein können und es mitunter auch sind. Aufschlussreiche Einblicke dazu bieten beispielsweise aktuelle Ergebnisse der „SPEAK!-Studie“, auf welche im weiteren Verlauf detaillierter eingegangen wird (vgl. Kapitel 4.2).

Inwiefern sexualisierter Gewalt durch präventive Angebote in Schulen begegnet wird, ist eine weitere Facette der differenzierten thematischen Betrachtung. Der UBSKM definierte Schulen als den zentralen Lebensraum für die Entwicklung von Konzepten zum Schutz vor sexualisierter Gewalt und der fortschreitenden Optimierung von Kinderschutz. Auch diese Zuschreibung wird genährt von der Annahme, dass der verpflichtende Besuch von Schulen zwangsläufig dazu führt, dass Lehrkräfte einen hohen Einfluss auf die Entwicklung von Schüler*innen haben (vgl. Arbeitsstab des UBSKM (2) o.J.).

\subsection{Sexualisierte Gewalt in der Lebenswelt von Kindern und Jugendlichen}

Im gesellschaftlichen Diskurs wird sexualisierte Gewalt seit einigen Jahren nicht mehr als randständiges Thema verhandelt. Viel mehr gibt es immer wieder breit angelegte Debatten zu Aufdeckungen, Intervention und Prävention sowie Themen wie Sexismus und sexualisierte Übergriffe in sozialen Netzwerken und einem adäquaten Umgang damit. Dass sexualisierte 
Gewalt auch in der Lebenswelt von Kindern und Jugendlichen kein Themenfeld ist, mit dem man nur in Ausnahmefällen in Berührung kommt, weisen zahlreiche Untersuchungen der letzten Jahrzehnte nach. Aufgrund verschiedenster zugrundeliegender Arbeitsdefinitionen, nach denen entsprechend die jeweiligen Untersuchungen konzipiert und durchgeführt wurden, ist eine exakte Aussage darüber, wie viele Heranwachsende tatsächlich von sexualisierter Gewalt betroffen sind und waren, nicht möglich. Wichtige Hinweise liefern konkrete Statistiken wie die Polizeiliche Kriminalstatistik (PKS), die beispielsweise für das Jahr 2016 mehr als 12.000 Fälle von sexuellen Übergriffen gegen Kinder gemäß der Paragrafen 176, 176a und 176b StGB verzeichnet. Betroffene Kinder und Jugendliche werden dabei mit $75 \%$ weiblich und 25\% männlich angegeben. Zusätzlich werden noch sexuelle Missbrauchsfälle von Schutzbefohlenen sowie 7.000 kinder- und jugendpornografische Vergehen gelistet. Zur Einordnung dieser Zahlen ist es notwendig zu reflektieren, dass die PKS lediglich die Fälle statistisch aufbereitet, welche den Ermittlungsbehörden auch tatsächlich bekannt geworden sind. Sie kann also lediglich Auskunft über das sogenannte Hellfeld geben (vgl. UBSKM 2017: 1f). Der UBSKM geht davon aus, dass pro Klasse etwa ein bis zwei Schulkinder von sexueller Gewalt betroffen sind (vgl. ebd.: 2). Neben der oftmals nicht erfolgten Aufdeckung erschwert mit zunehmendem Alter der Kinder und Jugendlichen ein weiteres Phänomen genaue Aussagen zur Prävalenz. Die Optimus-Studie, eine Schweizer Untersuchung publiziert von Schmidt aus dem Jahr 2014 mit 6749 befragten Schüler*innen neunter Klassen ( $n=6749)$, resümierte in einer Publikation, dass 39\% aller Übergriffe auf Heranwachsende (12-17 Jahre) von

Gleichaltrigen ausgehen:

Beziehung zum Täter bei Opfern in verschiedenen Altersgruppen, gemäss Institutionenumfrage (in Prozent)

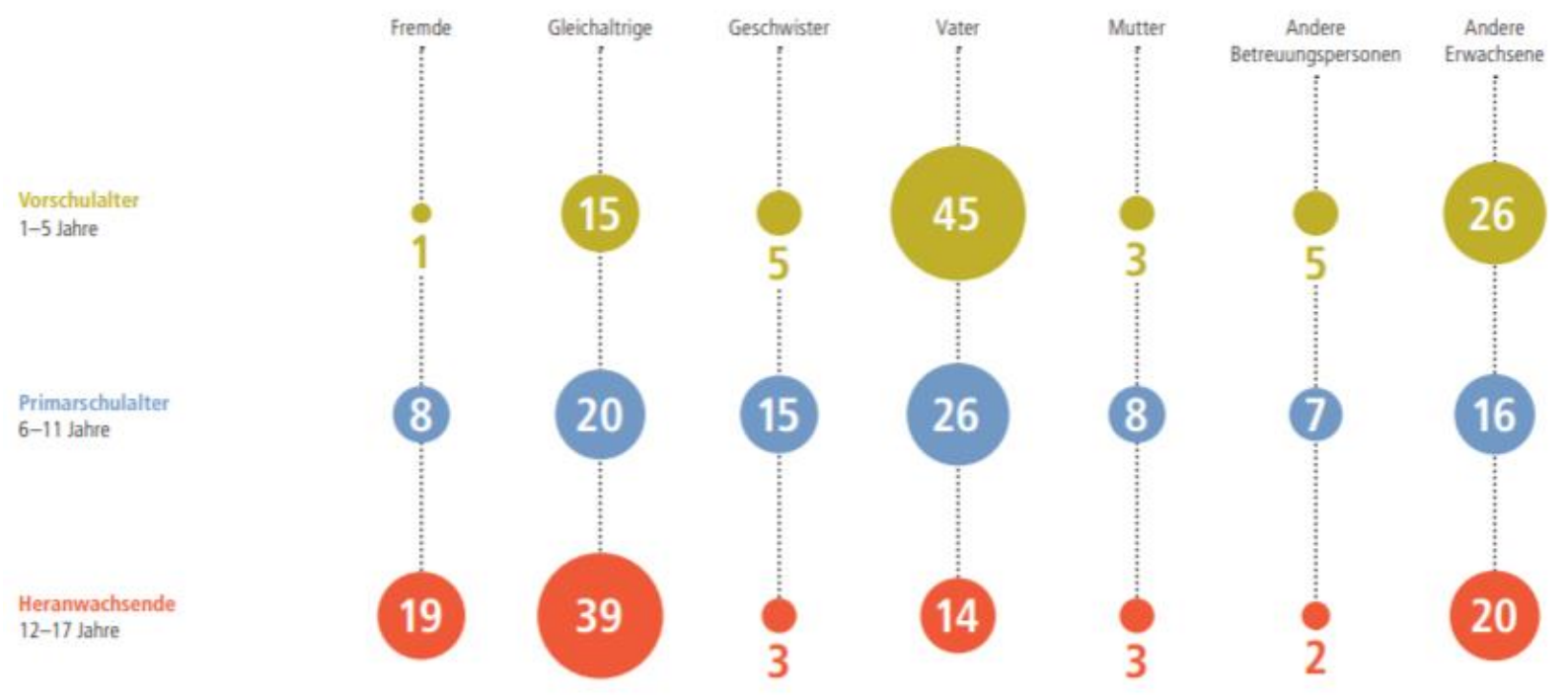

Abb. 10: Beziehung zum Täter bei Opfern in verschiedenen Altersgruppen

Quelle: Schmid 2012: 60. 
Es ist anzunehmen, dass durch das in Abbildung 10 veranschaulichte Phänomen die Abgrenzung von devianten zu strafrechtlich relevantem Verhalten zusätzlich erschwert wird sowohl für Ermittlungsbehörden als auch für die betroffenen Personen selbst.

Auf Ursachen, Auswirkungen, Folgen, möglichen Beeinträchtigungen und sonstige Aspekte für Betroffene sowie übergriffigen Personen soll an dieser Stelle nicht näher eingegangen werden. Eine kurze Betrachtung dieser Aspekte würde ihrer Bedeutsamkeit nicht gerecht werden, eine intensivere Auseinandersetzung jedoch über die Möglichkeiten der Arbeit hinausreichen.

Für die weiteren Abhandlungen dieser Untersuchung ist es jedoch notwendig, die obenstehende Abbildung noch einmal hinsichtlich ihrer Relevanz bezüglich präventiven Maßnahmen und ihrer Bedeutung für die Sexuelle Bildung im schulischen Kontext zu interpretieren. Je älter Kinder und Jugendliche werden, desto größer wird der Aktionsradius, in welchem sie wirken. Nicht zuletzt dadurch verändern sich die Personengruppen, von denen sexualisierte Übergriffe auf Heranwachsende unterschiedlichen Alters überwiegend ausgehen. Folgerichtig modifizieren sich damit auch die Herausforderungen für und Anforderung an Lehrkräfte und Sexuelle Bildung. Bis zum Schulalter muss Sexuelle Bildung insbesondere für und in Familien greifen und einen Beitrag dazu leisten, dass Kinder vor sexualisierter Gewalt (besser) geschützt werden. Ab Schulbeginn und in höheren Klassenstufen müssen „Interventions- und Präventionsstrategien (...) auf die unterschiedlichen Missbrauchserfahrungen von Kindern und Jugendlichen abgestimmt werden. (...) Ab dem Schulalter wird auch ein erweitertes Umfeld wichtiger und ab dem Jugendalter sollten Prävention und Intervention primär dort greifen, wo Jugendliche die meiste Zeit verbringen, nämlich oft außerhalb der Familie“ (vgl. Schmidt 2012: 59f).

\subsection{Die SPEAK!-Studie (Fokus Lebensraum Schule)}

Eine aktuelle Untersuchung, die „SPEAK!-Studie“, befragt ebenfalls Jugendliche zu ihren Erfahrungen mit sexualisierter Gewalt und gibt einen umfassenden Überblick dahingehend, wie junge Menschen sich im Kontext dieses Themas erleben. Auf einige Aspekte soll an dieser Stelle eingegangen werden. Besondere Aufmerksamkeit erlangte die Veröffentlichung der Studienergebnisse, da sie nicht nur von einer Betroffenenperspektive ausgeht, sondern auch den Blickwinkel der Jugendlichen aufgreift, die selbst sexuell übergriffig geworden sind oder sexualisierte Gewalt beobachtet haben (vgl. Pressestelle des Hessischen Kultusministeriums 2017).

Zunächst ermittelt auch die „SPEAK!-Studie“ hohe Prävalenzraten hinsichtlich des Erfahrens sexualisierter Handlungen gegen den eigenen Willen (vgl. Abbildung 11): 


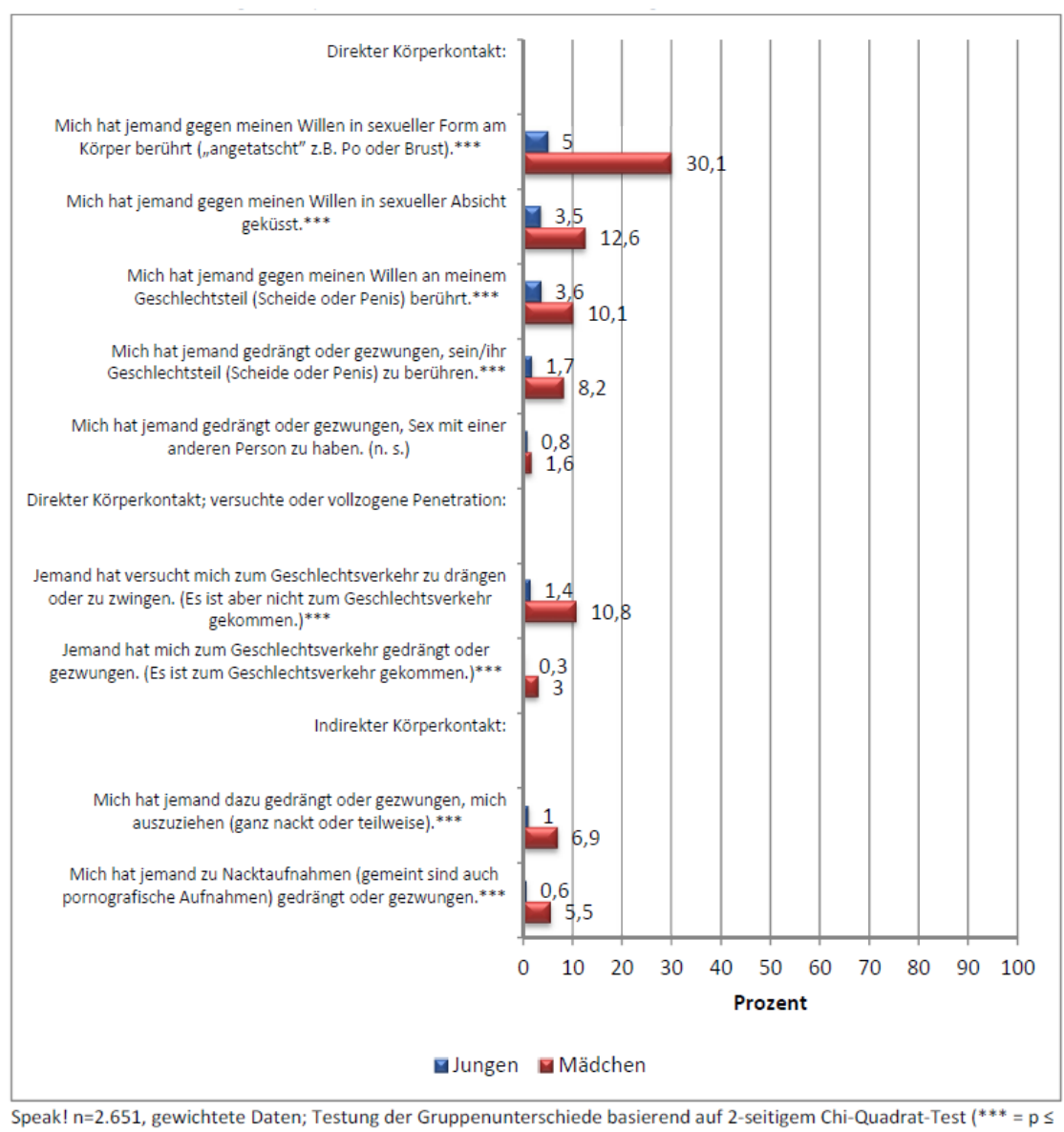

$.001 ;{ }^{* *}=p \leq .01 ; *=p \leq .05 ;$ n.s. $=$ nicht signifikant), Mehrfachantworten möglich.

\section{Abb. 11: Erfahrungen körperlicher sexualisierter Gewalt}

Quelle: Maschke/Stecher 2017: 9.

Mehr als 30\% der befragten Mädchen geben an, gegen ihren Willen Berührungen ihres Körpers in sexueller Form erlebt zu haben. Auch männliche Befragte verzeichnen Erfahrungen sexualisierter Gewalt auf körperlicher Ebene. Insgesamt sind es mehr als die Hälfte der befragten Neunt- und Zehnklässler*innen, die rückmelden, dass sie Erfahrungen mit direkten und/oder indirekten sexualisierter Übergriffen gemacht haben (Maschke/Stecher 2014: 9). Die Autor*innen der Studie konstituieren außerdem, dass die Wahrscheinlichkeit, von derartigen Übergriffen betroffen zu sein, mit zunehmendem Alter als Jugendliche*r steigt: „Während 13 Prozent der 14-Jährigen zu Protokoll geben, gegen den eigenen Willen an Po oder Brust angetatscht worden zu sein, steigt dieser Anteil bei den 17-Jährigen auf 25 Prozent. Von einem versuchten erzwungenen Geschlechtsverkehr berichten 4 Prozent der 14-Jährigen und 13 Prozent der 17-Jährigen“ (ebd.: 10). Diese Zahlen sprechen für den bereits zuvor angenommenen Zusammenhang zwischen einem größeren Wirkungskreis von Jugendlichen mit zunehmendem Alter und der ebenfalls größer werdenden Gefahr, von Übergriffen unter Gleichaltrigen betroffen zu sein. Dass Maschke und Stecher bezüglich der Prävalenzraten keine signifikanten Unterschiede hinsichtlich des besuchten Bildungsganges (und auch nicht 
bezüglich der sozialen Herkunft) nachweisen konnten, überrascht jedoch (vgl. Kapitel 4.5). Um die Lebenswelt von Jugendlichen und die Erfahrungen mit sexualisierter Gewalt in dieser möglichst umfassend abzubilden, haben Maschke und Stecher außerdem unter anderem nach Beobachtungen von sexualisierter Übergriffen gefragt. Dabei haben insgesamt $70 \%$ der Heranwachsenden angegeben, mindestens einmal sexualisierte Gewalt beobachtet zu haben - am häufigsten wurden dabei schriftliche oder verbale Angriffe in sexueller Form bemerkt, aber auch körperliche Berührungen gegen den Willen der betroffenen Person beobachteten rund ein Drittel der Jungen und Mädchen.

Neben eigener oder beobachteter Betroffenheit fokussiert die Studie außerdem auch darauf, inwieweit Jugendliche selbst übergriffig werden. Die erhobenen Ergebnisse finden sich in Abbildung 12.

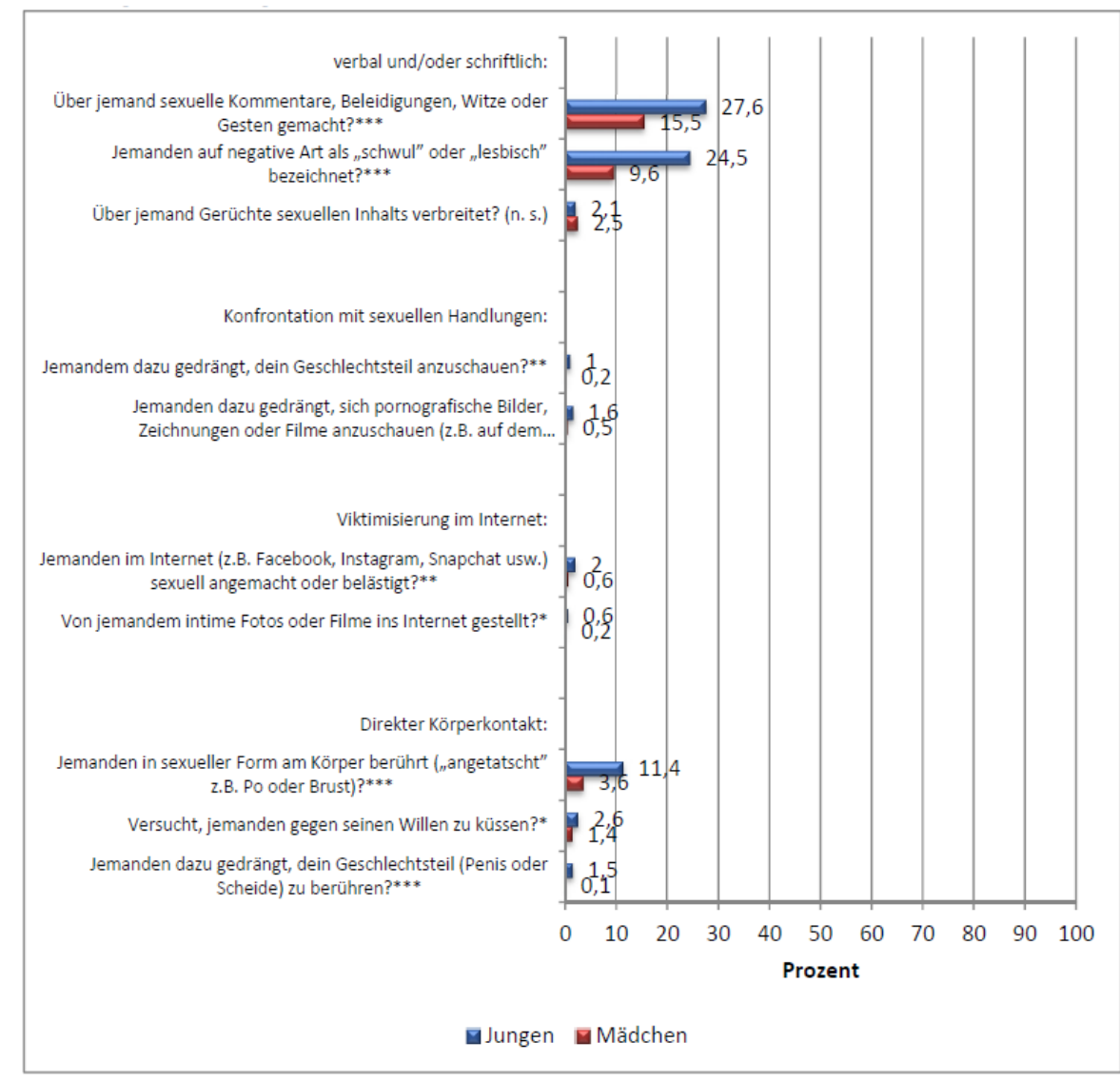

Speak! $\mathrm{n}=2.651$, gewichtete Daten; Testung der Gruppenunterschiede basierend auf 2-seitigem Chi-Quadrat-Test $\left({ }^{* * *}=\mathrm{p} \leq\right.$ $.001 ;{ }^{* *}=p \leq .01 ;{ }^{*}=p \leq .05 ;$ n.s. $=$ nicht signifikant), Mehrfachantworten möglich.

\section{Abb. 12: Ausübung von sexualisierter Gewalt}

Quelle: Maschke/Stecher 2017: 3.

Über ein Viertel der Befragten, konkret 28\%, melden zurück, bereits mindestens einmal selbst als Agressor*in² im Kontext sexualisierter Gewalt tätig gewesen zu sein. Auch bei der 
Betrachtung dieses Aspektes zeigt sich der schon bei der Betroffenheit angesprochene Alterseffekt - je älter die Jugendlichen, desto häufiger geben sie an, sexualisierte Gewalt ausgeübt zu haben (vgl. Maschke/Stecher 2017: 12f).

Auch hinsichtlich dessen, welche Orte von den Heranwachsenden als besonders risikoreich wahrgenommen werden, gibt die Studie Auskunft:

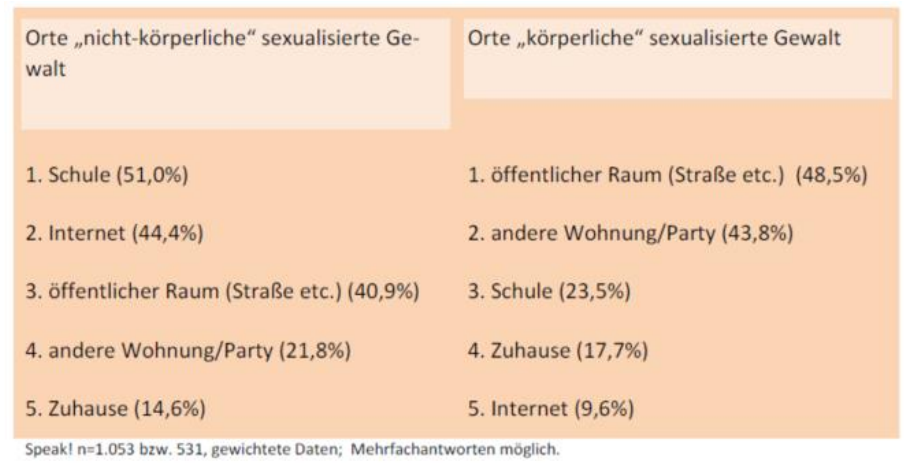

\section{Abb. 13: Risikoreiche Orte}

Quelle: Maschke/Stecher 2017: 15.

Sowohl hinsichtlich nicht-körperlicher sexualisierter Gewalt als auch bezüglich körperlicher sexualisierter Gewalt kommt der Schule als Ort für das Erleben beziehungsweise Ausüben derartiger Vorgänge eine hohe Bedeutung zu. Im Rahmen nicht-körperlicher sexualisierter Übergriffe ist sie sogar der risikoreichste Ort. Als häufigste Orte, an denen die Jugendlichen entsprechende Erfahrungen machen, werden für die Schule Klassenräume und Pausenhöfe benannt. Die Schüler*innen erleben den Lebensraum Schule demzufolge nicht als Schutzraum, sondern als Gefahrenzone (vgl. ebd.: 15). Nicht verwunderlich ist es daher, dass auch der*die Mitschüler*in häufig als die Person benannt wird, von der der Übergriff ausgeht:

\begin{tabular}{|c|c|}
\hline $\begin{array}{l}\text { Täterinnen und Täter nicht-körperliche se- } \\
\text { xualisierte }\end{array}$ & $\begin{array}{l}\text { Täterinnen und Täter körperliche sexuali- } \\
\text { sierte Gewalt }\end{array}$ \\
\hline \multicolumn{2}{|l|}{ Gewalt } \\
\hline 1. der „Fremde“ (41, 4\%) & 1. der „Fremde“ $(35,3 \%)$ \\
\hline 2. der Mitschüler $(35,8 \%)$ & 2. der Freund $(32,1 \%)$ \\
\hline 3. der Freund $(28,2 \%)$ & 3. der Mitschüler $(16,2 \%)$ \\
\hline 4. der Bekannte $(15,5 \%)$ & 4. der Bekannte $(15,9 \%)$ \\
\hline 5. der Ex-Partner (7,3\%) & 5. der Ex-Partner $(12,9 \%)$ \\
\hline
\end{tabular}

\section{Abb. 14: Übergriffige Personen}

Quelle: Maschke/Stecher 2017: 16.

Dass sowohl „Bekannte“ als auch „Fremde“ mit jeweils 75\% beziehungsweise $50 \%$ als 19 jährig oder jünger eingeschätzt wurden, ergänzt zum einen die Annahme, dass Übergriffe auf 
Jugendliche am ehesten von anderen Jugendlichen ausgehen. Zum anderen verdeutlicht es nochmals, dass Schüler*innen auch als potentiell übergriffige Personen von Sexueller Bildung adressiert werden müssen. Entsprechend der Regelungen im jeweiligen Bundesland sind junge Menschen bis circa 18 Jahre grundsätzlich schulpflichtig ${ }^{3}$. Der Großteil der von den befragten Personen benannten Aggressor*innen ist demzufolge selbst noch Schüler*in oder bis vor kurzem schulpflichtig gewesen und damit im Lebensraum Schule zu verorten. Die Ergebnisse der „SPEAK!-Studie“ im Gesamtüberblick zeichnen ein bedenkliches Bild der Lebenswelt Jugendlicher und der Präsenz von sexualisierter Gewalt in dieser. Zwar setzt die Studie beziehungsweise der verwendete Fragebogen außerordentlich niedrigschwellig an und weist in einer Gesamtrechnung $81 \%$ junge Menschen aus, welche Berührung mit sexualisierten Übergriffen gemacht haben (vgl. Maschke/Stecher 2017: 14). Dennoch bleibt der Bedarf an Sexueller Bildung und gleichzeitig die Notwendigkeit von Kompetenzvermittlung an Lehrkräfte, die täglich mit Jugendlichen im Kontakt sind, unverkennbar. Sexualisierte Gewalt - ob im eigenen Erleben, aus beobachtender Perspektive oder als ausführende Person - ist eine Thematik, mit der sich eine Vielzahl der Heranwachsenden früher oder später auseinandersetzt. Umso notwendiger ist es, Teenagern mit geeigneten Maßnahmen und Methoden eine intensive Auseinandersetzung - außerhalb des direkten Erlebens - zu ermöglichen. Sowohl die hohen Prävalenzraten als auch die Auskünfte der befragten Schüler*innen hinsichtlich risikoreicher Orte und häufigster übergriffiger Personen lässt annehmen, dass die Situationen, in denen sexualisierte Gewalt erlebt wird, keinen vorhersehbaren und überdurchschnittlich gefährlichen oder besonderen Charakter haben müssen, um zu solchen zu werden. Es ist umso wahrscheinlicher, dass es sich um Erfahrungen im Rahmen eines üblichen Alltags von Jugendlichen handelt, in welchem der Lebensraum Schule bis etwa zum 18. Lebensjahr einen wesentlichen Bestandteil einnimmt. Entsprechend dringend notwendig ist es, dass Lehrkräfte ausreichend professionalisiert sind, um derartige Prozesse zu erkennen, anzuleiten und entsprechend intervenierend und/oder präventiv zu handeln.

\subsection{Schulen als Tatorte}

In den voranstehenden Kapiteln ist die Schule als Lebensraum definiert worden, in dem sexualisierte Übergriffe stattfinden. In der historischen Betrachtung wurde diesem Umstand lange wenig Rechnung getragen. Heinzel und Prengel halten in diesem Zusammenhang fest,

${ }^{3}$ Die meisten Bundesländer legen eine Pflichtanzahl an Schuljahren fest, welche Schüler*innen absolvieren müssen. Bis auf wenige Ausnahmen ist so eine Schulpflicht bis mindestens zur Vollendung des 18. Lebensjahres die Regel. 
dass „keine historischen Untersuchungen zu sexualisierter Gewalt an Schulen vor[liegen], das Thema wird weitgehend tabuisiert“ (Heinzel/Prengel 2018: 416f). Seit der zweiten Frauenbewegung in den 1960er bis 1980er Jahren steht der sexuelle Missbrauch an Kindern (allerdings mit Fokus auf Vorfälle innerhalb der Herkunftsfamilie) in der öffentlichen Diskussion. Im Zuge dessen wurde auch begonnen, informative Angebote zu sexuellem Missbrauch sowie entsprechenden Präventions- und Interventionsmaßnahmen für Lehrkräfte vorzuhalten (vgl. ebd: 217f).

Größere Initiativen - auch im wissenschaftlichen Diskurs - brachte vor allem die Thematik Gewalt an Schulen mit sich, welche seit den 1990er Jahren vielfach öffentlich verfolgt wurde - allerdings ebenfalls, ohne umfassender auf sexualisierte Gewalt zu fokussieren. Wenn das Phänomen punktuell thematisch aufgegriffen wurde, wurden vor allem die sexualisierten Übergriffe ausgespart, welche von Lehrkräften auf Schüler*innen ausgehen. Dies änderte sich mit den Disclosure-Prozessen ${ }^{4}$ seit dem Jahr 2010 (vgl. Kapitel 1.3).

\subsubsection{Sexualisierte Gewalt im Lebensraum Schule}

Im schulischen Kontext treten verschiedene Formen sexualisierter Gewalt auf. Bezüglich des Erscheinungsbildes ist anzunehmen, dass sexualisierte Gewalt in schulischen Institutionen in allen Facetten vorkommt, in denen sie auch in sonstigen Lebensfeldern auftritt. Es gibt zahlreiche fachliche Auseinandersetzungen mit den Erscheinungsformen des Phänomens, weshalb an dieser Stelle davon Abstand genommen wird, diese intensiver zu betrachten. Zusätzlich muss jedoch nach Personengruppen unterschieden werden, die betroffen sind beziehungsweise die Übergriffe ausüben. Im Folgenden soll dieser Aspekt näher hinterfragt werden.

Innerhalb der Auseinandersetzung mit der Thematik wurde bereits frühzeitig in den Blick genommen, dass Schüler*innen sexuell übergriffig gegenüber Gleichaltrigen, also untereinander, werden. So konstatiert beispielsweise Bundschuh, dass „nicht zuletzt die neuen Medien, die neue Möglichkeiten für die Ausübung der sexualisierten Gewalt bieten, (...) zu einer veränderten Problemsituation geführt [haben], die zunehmend auch als solche wahrgenommen wird“ (Bundschuh 2010: 21). Schüler*innen erleben sexuelle Angriffe zum Beispiel bei der Nutzung von Messangerdiensten wie Facebook oder WhatsApp in Form von direkten verbalen Angriffen, aber auch durch das Anfertigen, Zeigen oder Verbreiten von intimen Videoaufnahmen gegen den eigenen Willen. Eine besondere Herausforderung stellen

\footnotetext{
4 Disclosure meint den „Prozess, der vom Schweigen über erlebten sexuellen Missbrauch zum Sprechen führt" (Kavemann et al. 2016: 70). Es handelt sich um einen lebenslangen Prozess, in dem die betroffene Person aktiv handelt und eine Entscheidung für oder gegen das Offenlegen trifft (vgl. ebd.)
} 
bei den Kindern und Jugendlichen die Adressat*innen Sexueller Bildungsangebote dar, die selbst traumatische sexualisierte Gewalterfahrungen gemacht haben und im weiteren Verlauf ihres schulischen Werdeganges selbst sexuell grenzüberschreitend werden. Diese Aggressor*innengruppe hat eine besondere Bedürftigkeit, der sensibel nachgegangen werden muss, ohne die Beteiligten zu überfordern. Gerade in der Aushandlung von inklusiven Bildungseinrichtungen muss diskutiert werden, (in)wie(weit) diese jungen Menschen im Schulalltag einer Regelschule integriert werden können, ohne sich selbst oder andere Personen zu gefährden (vgl. Siedenbiedel 2018: 553f).

Neben den Übergriffen unter Gleichaltrigen sind die Aufdeckungsvorgänge seit 2010 ein unbestreitbarer Nachweis dafür, dass es selbstverständlich auch zu Übergriffen von Lehrpersonen auf Schüler*innen kommt. Kinder und Jugendliche in schulischen Institutionen stehen in einem besonderen Abhängigkeits- und Machtverhältnis zu den sie in den Einrichtungen umgebenden Erwachsenen. Sexualisierte Übergriffe von Lehrkräften auf Schüler*innen sind häufig nicht eindeutig als solche nachzuweisen, da sie getarnt als pädagogische Maßnahmen diffus bleiben und dadurch in ihrer Notwendigkeit vermeintlich argumentativ begründet werden können (vgl. Bründel 2011: 20). Prengel et al. führten umfangreiche Studien in Form von Beobachtungen durch und konnten etwa sechs Prozent der Interaktionen zwischen Lehrkräften und Schüler*innen als psychisch mitunter stark verletzend identifizieren. In den Erhebungen kam es aber kaum zu Beobachtungen von sexualisierten Handlungen der Lehrer*innen gegen die Schüler*innen (vgl. Heinzel/Prengel 2018: 419). Dennoch melden Kinder und Jugendliche Vorfälle dieser Art durchaus zurück. In Schulen tätige Pädagog*innen, die sexualisierte Übergriffe planen oder bereits vollziehen, verstehen es jedoch - ähnlich wie andere Täter*innen - mögliche verdächtige Momente erfolgreich von sich zu weisen. Aufgrund ihrer institutionellen Position verfügen sie über Mittel und Möglichkeiten, die betroffenen Kinder und Jugendlichen von der Gruppe der übrigen Schüler*innen zu isolieren und sie damit in eine Sonderrolle zu bringen (vgl. Münder/Kavemann 2010: 7). In anderen Fällen sind körperliche Berührungen tatsächlich Bestandteil der pädagogischen (Unterstützungs-)Handlung. Insbesondere im Sportunterricht, aber auch in anderen schulalläglichen Situationen sind derartige Konstellationen vorstellbar. Dies alles erschwert eine verlässliche Untersuchung der Prävalenzraten sexualisierter Übergriffe von Lehrkräften auf Schüler*innen. Im Jahr 2013 beschäftigte sich die Kultusministerkonferenz (KMK) intensiv mit der Thematik. In ihren Handlungsempfehlungen identifiziert sie sexuelle Grenzüberschreitungen von Lehrer*innen gegenüber anvertrauten Heranwachsenden als grundlegendes Fehlverhalten hinsichtlich der vorgesehenen dienstlichen Tätigkeiten. Die Verantwortlichen weisen außerdem ausdrücklich darauf hin, dass selbst ein Fehlen einer eindeutig nachweisbaren Strafbarkeit des Vorkommnisses nicht zwangsläufig vor härtesten Sanktionen als disziplinarrechtliche Maßnahmen schützt. Konkret 
bedeutet dies, dass Lehrkräfte im härtesten Fall auch mit der Entfernung aus dem Beamtenverhältnis belangt werden können, selbst wenn Ermittlungsbehörden nicht nachweisen, dass eine Straftat im Sinne des Strafrechtes vorliegt (vgl. KMK 2013: 2f).

Trotz dessen Ermittlungsbehörden bereits vor 2010 von einschlägigen Fällen Kenntnis erlangt haben, erfolgte im gesellschaftlichen Diskurs keine Konfrontation mit dieser Wahrheit. Dies ist insofern verwunderlich, als dass mindestens seit dem Jahr 1943 sexualisierte (Gewalt)Handlungen von Lehrkräften gegenüber ihren Schüler*innen im Rahmen des Verbots von sexuellem Missbrauch von Schutzbefohlenen gesetzlich ausgeschlossen werden. Zwar gab es seitdem sowohl inhaltliche Anpassungen als auch Veränderungen hinsichtlich des entsprechenden Schutzalters (inzwischen liegt es bei 18 Jahren), dennoch scheint die Schutzbedürftigkeit von Schüler*innen grundsätzlich als staatliche Aufgabe betrachten worden zu sein (vgl. Fuchs o. J.). Dies ist jedoch kein rein nationales Versäumnis. Auch im internationalen Feld gab es bis zum Jahr 2010 keine gesicherten und weitreichenden Kenntnisse zu Fallzahlen und Ausprägungen derartiger Vorkommnisse (vgl. Bundschuh 2010: 21). Eine erste größere Studie dieser Art führte das Deutsche Jugendinstitut (DJI) durch. Der im Jahr 2011 veröffentlichte Abschlussbericht weist rund ein Fünftel aller teilnehmenden Schulen aus, die in der durchgeführten Befragung angaben, dass innen in den vergangenen drei Jahren mindestens ein Verdachtsfall bekannt wurde. Eingeschlossen in diese Verdachtsfälle waren sowohl sexualisierte Übergriffe unter Schüler*innen als auch Übergriffe von Lehrpersonen auf die anvertrauten Kinder und Jugendlichen. In der Mehrheit aller Fälle wurde dabei von einer einzelnen betroffenen Person berichtet. Beachtet werden muss, dass die Untersuchung tatsächlich nur dahingehend untersucht, inwieweit Fälle offengelegt wurden. Ähnlich wie hinsichtlich allgemeiner Prävalenzraten von sexualisierter Gewalt ist anzunehmen, dass die Dunkelziffer des tatsächlichen Vorkommens um ein Vielfaches höher liegt (vgl. Helming et. al 2011: 240ff).

Aus meiner Sicht gibt es eine weitere bisher vernachlässigte Gruppe potentiell von Übergriffen in schulischen Institutionen betroffener Personen: die Lehrkräfte selbst. Es ist wahrscheinlich, dass auch sie häufig Ziel sexualisierter Angriffe sind - sowohl von Schüler*innen als vermutlich auch innerhalb des Kollegiums. Da diese Arbeit jedoch auf Kinder und Jugendliche und ihren Schutz vor sexualisierter Gewalt fokussiert, soll diese These lediglich Erwähnung finden ohne tiefergehend betrachtet zu werden. Gleichzeitig darf auch dieser Aspekt in der Adressierung von Sexueller Bildung an Schüler*innen nicht in Vergessenheit geraten. Da diese innerhalb einer mehr oder weniger hierarchischen Institution - wie Schulen es sind - nur eine sehr begrenzte Möglichkeit haben, hinsichtlich ihrer Wünsche wirksam zu werden, sind sexualisierte Übergriffe ein vorstellbares Mittel der Selbstermächtigung. An anderer Stelle sollte dies demzufolge kritisch hinterfragt, untersucht und diskutiert werden. 


\subsubsection{Risikofaktoren für sexualisierte Übergriffe in schulischen Institutionen}

Eine generelle Betrachtung von Risikofaktoren für sexualisierte Übergriffe in schulischen Institutionen ist in mehrfacher Hinsicht wichtig. Einen wesentlichen Grund dafür führen Bienstein et. al an, die herausstellen, dass Einrichtungen, in denen sexualisierte Gewalt auftritt, die Fortdauer und Verstetigung der Übergriffe ermöglichen, solange die Vorfälle als Einzelfälle deklariert werden und keine Betrachtung der strukturellen Voraussetzungen vorgenommen wird (vgl. Bientstein et. al 2018: 211). Daraus hervor geht die Annahme, dass es unter anderem institutionelle Bedingungen sind, die sexualisierte Grenzverletzungen begünstigen oder ihnen vorbeugen.

Enders gibt konkrete Auskünfte darüber, welche institutionellen Strukturen sexualisierte Gewalt besonders begünstigen. Zunächst benennt sie autoritäre und unklare Strukturen auf Leitungsebene als zentralen Risikofaktor (vgl. Enders 2012: 132). Da Entscheidungen in Einrichtungen mit diesem Leitungsmuster weniger auf fachlichen Standards basieren als mehr persönlich begründet sind, haben potentiell übergriffige Personen durch den Aufbau von persönlichen Beziehungen zur Leitung die Möglichkeit, Übergriffe erfolgreich zu verbergen (vgl. ebd.). Gleiches gilt, wenn die Schulleitung ihre Rolle als Führungskraft nicht oder nur unzureichend erfüllt, da die fachliche Kontrolle der institutionellen Abläufe durch die Leitungsebene fehlt (vgl. ebd.). Beide Leitungsstrukturen münden häufig darin, dass Vorfälle nicht verfolgt oder zuständigen Aufsichtsbehörden nicht gemeldet werden und begünstigen Täter*innenverhalten (vgl. ebd.: 133).

Einen weiteren wesentlichen Risikofaktor sieht Enders darin, wenn sich Schulen als geschlossenes System verstehen. So konstituiert sie, dass „blindes Vertrauen und eine starke Einbindung in den institutionellen Alltag geschlossener Systeme (...) Kindern und Jugendlichen und vor allem auch Eltern [erschwert], eine gesunde kritische Distanz zu der Einrichtung (...) aufrechtzuerhalten und Hinweise auf sexuelle Übergriffe (...) wahrzunehmen“ (Enders 2012: 134f). Die für das geschlossene System geltenden spezifischen Verhaltensund Werteideale führen häufig zu risikobehafteten Abhängigkeitsverhältnissen (vgl. ebd.). Achten Schulen nicht auf eine angemessene Trennung zwischen beruflichen und privaten Kontakten, fördert dies Distanzlosigkeit und damit ebenfalls sexualisierte Grenzverletzungen (vgl. ebd.).

Auch eine mangelnde Partizipation der Schüler*innenschaft im Schulgeschehen kann Raum für sexualisierte Gewalt schaffen. Da sich die Inhalte der Erziehung dabei zumeist nach den Wertvorstellungen der Erwachsenen richten, werden altersentsprechende Bedürfnisse der Kinder und Jugendlichen vernachlässigt. Damit geht häufig auch das Fehlen einer adäquaten Förderung der Schüler*innen einher. Dies begünstigt Abhängigkeiten und damit für Grenzverletzungen anfällige Beziehungen, da es für übergriffige Personen durch wenig pädagogisches Engagement verhältnismäßig einfach ist, in einen vertrauensvollen Kontakt zu 
Eltern und Schüler*innen zu treten (vgl. Enders 2012: 140f).

Durch traditionelle Rollenbilder verstärken pädagogische Konzepte die Schwächung der Widerstandsfähigkeit von Kindern und Jugendlichen. Verfügen sie jedoch über weniger Widerstandsfähigkeit, fällt es ihnen ebenfalls schwerer, sich gegen übergriffige Personen zu wehren (vgl. ebd.: 141). In Verbindung mit nicht ausgereiften oder fehlenden sexualpädagogischen Konzepten minimiert sich damit das Risiko der Aufdeckung von sexualisierten Übergriffen, da den Schüler*innen entweder gänzlich die Sprache fehlt um auszudrücken, welche Erfahrungen sie gemacht haben oder sie keine vertrauensvollen Räume finden, in denen ein offenes Sprechen über Sexualität möglich ist. Eine mangelnde Auseinandersetzung mit ihrem Körper, ihren Empfindungen und Bedürfnissen führt dazu, dass Kinder und Jugendliche Grenzverletzungen schlechter als solche wahrnehmen können (vgl. ebd.: 142f). Vor allem, wenn die Grenzen zwischen verschiedenen Generationen missachtet werden und beispielsweise die Schüler*in-Lehrer*in-Beziehung insofern vertraulich wird, dass auch die Lehrkräfte sich mit persönlichen Themen ihren Schüler*innen anvertrauen, vermischen sich auch die Grenzen von Intimität und wird es den Heranwachsenden zusätzlich erschwert, Akte des Vertrauens als Grenzverletzung zu erkennen (vgl. ebd.: 142). Eine adäquate Partizipation von Schüler*innen beinhaltet auch, dass sie interne und externe Ansprechpersonen haben, an die sie sich mit Beschwerden wenden können. Nur mit einem funktionierenden, das heißt strukturell geregelten Beschwerdemanagement können Kinder und Jugendliche sicher sein, dass ihre Nöte ernst genommen und an die zuständigen Personen herangetragen werden (vgl. ebd.: 142f).

\subsection{Förderschulen als besonders gefährdende Institution}

Die Situation von Schüler*innen mit sonderpädagogischem Förderbedarf muss in mehrfacher Hinsicht genauer betrachtet werden. Kinder und Jugendliche mit kognitiven Beeinträchtigungen, Lernschwierigkeiten, psychischen Krankheiten oder Störungen im Sozialverhalten sind insofern besonders vulnerabel, als dass sie sich in einem besonderen Gefährdungsfeld bewegen. Dieses ist gekennzeichnet von einer größeren Abhängigkeit zu dem sie umgebenden sozialen Umfeld, fehlenden oder veränderten Kommunikationskompetenzen, Erfahrungen von Diskriminierung und Ausgrenzung beziehungsweise Isolation. Aufgrund dieser besonderen Bedingungen können sich junge Menschen mit Beeinträchtigungen schlechter gegen sexualisierte Übergriffe wehren als Gleichaltrige. Dies schließt sowohl sexualisierte Gewalt im schulischen Kontext als auch sexualisierte Angriffe außerhalb der Institution Schule ein. Begünstigend dabei muss nicht immer (nur) ein Mangel an Sexueller Bildung der betroffenen Personen sein. Viel mehr können auch oder gerade institutionelle Rahmenbedingungen Risikofaktoren für das Vorkommen 
sexualisierter Gewalt sein (vgl. Lache 2018: 297f).

Generelle Risikofaktoren in schulischen Kontexten wurden bereits beschrieben (vgl. Kapitel 4.4). Inwiefern Förderschulen noch einmal als besonders gefährdend angesehen werden können, soll hier noch einmal erläutert werden.

Allgemeinengültig führen Chodan et. al aus, dass „der öffentliche Diskurs durch ein Hinzukommen eines weiteren Tabus, dem der Sexualität Behinderter, begrenzt" wird (Chodan et. al 2014: 408). Dies allein erschwert eine umfassende gesellschaftliche Debatte der Thematik und die Auseinandersetzung mit Lösungsstrategien. Gleichzeitig geht damit einher, dass sie weniger Beachtung findet und Gefahren weniger wahrgenommen beziehungsweise als solche identifiziert und Vorfälle seltener aufgedeckt werden. In Bezug auf Schulen mit Förderschwerpunkten ist eine explizite Betrachtung der Situation jedoch im besonderen Maße angezeigt. So konstituieren Rabold und Baier, dass Förderschüler*innen zum einen in größerem Maße gefährdet sind, diverse Formen devianten Verhaltens zu zeigen, zeitgleich jedoch auch einem höheren Risiko unterliegen, in der Schule (und in der Familie) von Gewalt betroffen zu sein (vgl. Rabold / Baier 2008: 121). Ergänzend dazu wiesen im Jahr 2011 Helming et. al darauf hin, dass es an Förderschulen „im Vergleich $\mathrm{zu}$ anderen Schulen insgesamt deutlich mehr Verdachtsfälle auf institutionellen sexuellen Missbrauch“ gibt (Helming et. al 2011: 243). Diese beziehen sich jedoch nicht auf Übergriffe durch Beschäftigte, sondern auf von den Schulen häufiger berichtete sexuelle Grenzverletzungen durch Gleichaltrige. Darüber hinaus kommt es an Förderschulen ebenfalls häufiger zu Disclosure-Prozessen von sexualisierter Gewalt außerhalb der Schule, was bedeutet, dass Lehrer*innen in einem höheren Maß gefordert sind, Verdachts- und Aufdeckungsfällen zu begleiten (vgl. ebd.).

Die Ergebnisse der Erhebung Helmings et. al lassen den Schluss zu, dass Förderschulen in einem noch höheren Maße als andere Schulformen gefordert sind, präventiv gegen sexualisierte Gewalt vorzugehen und zum Schutz von Kindern und Jugendlichen beizutragen. Dies gestaltet sich insofern schwierig, als dass für Kinder mit sonderpädagogischen Förderbedarfen noch weniger bewährte Präventionsangebote und -konzepte vorliegen als für Kinder ohne Beeinträchtigungen (vgl. ebd.).

Auch die Studie "Sexualisierte Gewalt in der Erfahrung Jugendlicher“ ( $n=264)$, eine Erweiterungsstudie der „SPEAK!-Studie“ aus dem Jahr 2018, untersuchte in ihrem Forschungsprozess, ob Förderschüler*innen im Vergleich zu Schüler*innen anderer Schulformen häufiger sexualisierte Gewalt erleben (vgl. Maschke / Stecher 2018: 4).

Bereits die bis hierhin dargestellten Erkenntnisse weisen darauf hin, dass die Situation von Förderschulen, Förderschüler*innen und damit auch Lehrkräften an Schulen mit sonderpädagogischem Förderbedarf einer umfassenden Betrachtung bedarf. Eine intensive Auseinandersetzung würde über den Rahmen dieser Masterarbeit hinausführen und kann 
deshalb nicht vorgenommen werden. Dennoch soll eine kleine Auswahl der Ergebnisse der „SPEAK!-Erweiterungsstudie“ die Brisanz der Thematik verdeutlichen und zu einer wissenschaftlichen Bearbeitung an anderer Stelle anregen:

\begin{tabular}{|c|c|c|c|c|c|}
\hline & \multicolumn{3}{|c|}{ (Zusammengefasste) Förderschwerpunkte } & \multirow{2}{*}{$\begin{array}{c}\text { Förder- } \\
\text { schule Ge- } \\
\text { samt }\end{array}$} & \multirow{2}{*}{$\begin{array}{c}\text { Hauptstudie } \\
\text { Speak! }\end{array}$} \\
\hline & Lernen & $\begin{array}{l}\text { Hören und } \\
\text { Sehen }\end{array}$ & $\begin{array}{c}\text { Sprache und emo- } \\
\text { tionale/soziale } \\
\text { Entwicklung }\end{array}$ & & \\
\hline Gesamt & 30 & 34 & 24 & 30 & 23 \\
\hline $\begin{array}{l}\text { Weibl. Ju- } \\
\text { gendliche }\end{array}$ & $49 * * *$ & 44 & 31 & $45^{* * *}$ & $35^{* * *}$ \\
\hline $\begin{array}{l}\text { Männl. Ju- } \\
\text { gendliche }\end{array}$ & 17 & 23 & 21 & 19 & 10 \\
\hline
\end{tabular}

Abb. 14: Prävalenz körperliche sexualisierte Gewalt (Förderschüler*innen)

Quelle: Maschke/Stecher 2018: 17.

Wie Abbildung 16 verdeutlicht, hat etwa ein Drittel der befragten Jugendlichen körperliche sexualisierte Gewalt erlebt. Im Vergleich zu den Ergebnissen der Hauptstudie bestätigt sich damit die Annahme, dass Schüler*innen mit Förderbedarf einem höheren Risiko unterliegen, von sexualisierter Übergriffen betroffen zu sein. Über die drei Förderschwerpunkte Lernen, Hören und Sehen sowie Sprache und emotionale/soziale Entwicklung hinweg sind es insbesondere Mädchen, die Erfahrungen mit körperlicher sexualisierter Gewalt angeben. Auch die Frage danach, ob sie bereits sexualisierte Gewalt ausgeübt haben, bestätigen Förderschüler*innen (geringfügig) häufiger als Jugendliche, die andere Schulformen besuchen (vgl. Abbildung 17).

\begin{tabular}{|c|c|c|c|c|c|}
\hline & \multicolumn{3}{|c|}{ (Zusammengefasste) Förderschwerpunkte } & \multirow{2}{*}{$\begin{array}{l}\text { Förder- } \\
\text { schule Ge- } \\
\text { samt }\end{array}$} & \multirow{2}{*}{$\begin{array}{c}\text { Hauptstudie } \\
\text { Speak! }\end{array}$} \\
\hline & Lernen & $\begin{array}{l}\text { Hören und } \\
\text { Sehen }\end{array}$ & $\begin{array}{c}\text { Sprache und emo- } \\
\text { tionale/soziale } \\
\text { Entwicklung }\end{array}$ & & \\
\hline Gesamt & 31 & 37 & 27 & 31 & 28 \\
\hline $\begin{array}{l}\text { Weibl. Ju- } \\
\text { gendliche }\end{array}$ & 29 & 30 & 19 & 28 & $21 * * *$ \\
\hline $\begin{array}{l}\text { Männl. Ju- } \\
\text { gendliche }\end{array}$ & 33 & 42 & 31 & 34 & 36 \\
\hline
\end{tabular}

Abb. 15: Prävalenz Ausübung sexualisierter Gewalt (Förderschüler*innen)

Quelle: Maschke/Stecker 2018: 24.

Da die prozentual übergriffig gewordenen Jugendlichen jedoch noch immer etwa einem Drittel der Schüler*innenschaft entsprechen, stehen Lehrkräfte auch an Förderschulen der Herausforderung gegenüber, auch sexuell grenzverletzende Kinder und Jugendliche in den Blick zu nehmen und im Umgang mit ihnen handlungsfähig zu sein. 


\subsection{Prävention sexualisierter Gewalt im Schulalltag}

Mit den Aufdeckungsprozessen seit 2010 hat auch die gesellschaftliche Auseinandersetzung zur Prävention sexualisierter Gewalt eine neue Stufe erreicht. Insbesondere der Institution Schule als zum einen Aufdeckungsort zahlreicher Missbrauchsfälle ${ }^{5}$ und zum anderen Mitverantwortliche für Fragen der Sexualaufklärung beziehungsweise Sexualerziehung kommt dabei eine zentrale Rolle zu (vgl. Glammeier 2018: 197). Inzwischen gibt es bereits zahlreiche Publikationen in Form von Handlungsleitfäden oder informierenden Broschüren, denen Lehrer*innen praktische Tipps für ihre tägliche Arbeit entnehmen können (vgl. ebd.). Das BMBF-Forschungsprojekt „Sexualisierte Übergriffe und Schule - Prävention und Intervention" untersuchte unter anderem die Frage, wie die Institution Schule hinsichtlich ihrer konzeptionellen Bedingungen ausgestattet ist, um die Prävention sexualisierter Gewalt voranzubringen. Neben der zentralen Erkenntnis, dass es auf Seiten der Lehrkräfte große Wissenslücken gibt, zeigen die Forschungsergebnisse auch auf struktureller Ebene erhebliche Missstände auf (vgl. Glammeier 2018: 200ff).

Je nach Schulform unterscheidet sich die Intensität, mit der präventiv im Schulalltag gearbeitet wird. In der Erhebung Glammeiers $(n=976)$ wird dieser Punkt durch die Erfahrung der befragten Lehrkräfte mit selbst geleisteter Präventionsarbeit abgebildet. Insbesondere in Grundschulen und Förderschulen haben Lehrer*innen vergleichsweise häufig bereits präventive Inhalte angeboten, nämlich in 53\% (Förderschullehrkräfte) beziehungsweise $72 \%$ (Grundschullehrkräfte) der Fälle. Im deutschsprachigen Raum gibt es bisher kaum wissenschaftlich evaluierte Präventionsprogramme (vgl. Bauer et al. 2018: 182). Aus der Analyse bekannter Präventionsprojekte entnehmen Bauer et al., dass präventive Angebote in Grundschulen unter anderem auf das Sexualwissen von Kindern zielen, welches beispielsweise Wissen über sexuelles Verhalten und Sprache in Form von Begriffen und Bezeichnungen einschließt. Mehrheitlich fokussieren die präventiven Inhalte darauf, dass Kinder Selbstschutzkompetenzen erwerben, um sich zukünftig besser gegen sexualisierte Übergriffe zur Wehr setzen zu können (vgl. ebd.).

An Gesamt-, Haupt- und Realschulen haben zwischen einem Viertel und einem Fünftel der befragten Lehrkräfte bereits Erfahrungen mit von ihnen durchgeführten Präventionsangeboten (vgl. Glammeier 2018: 202). Noch weniger vertraut mit präventiven Maßnahmen sind Lehrkräfte an Gymnasien und Berufsschulen, die nur zu 15\% (Gymnasien) beziehungsweise $5 \%$ bereits selbst Angebote umgesetzt haben.

\footnotetext{
${ }^{5}$ Die Aufdeckungen konzentrierten sich vorrangig auf Internate, jedoch fokussiert die gesellschaftliche und auch wissenschaftliche Auseinandersetzung inzwischen auch Regel- und Förderschulen ohne angeschlossene Internate.
} 
Die Erhebung führt Glammeier zu dem Schluss, dass es bisher keine flächendeckende schulische Präventionsarbeit gibt (vgl. ebd.: 207). Neben den Kompetenzen der Lehrkräfte, die diese im Schulalltag durchführen würden, fehlt es jedoch insbesondere an grundlegenden und strukturell verankerten Standards und Leitlinien zur Prävention sexualisierter Gewalt an Schulen (vgl. Bauer et. al 2018: 181).

Um den Missständen der institutionellen Rahmenbedingungen entgegenzuwirken, hat die Bundesregierung im Jahr 2016 die Bundesinitiative „Schule gegen sexuelle Gewalt" initiiert (vgl. Glammeier 2018: 197). Die Initiative soll Schulen aller Schulformen dabei unterstützen, sich mittelfristig in den Prozess zu begeben, ein schuleigenes Schutzkonzept zu erstellen. Der UBSKM beschreibt Schutzkonzepte zur Prävention und Intervention als „ein Zusammenspiel aus Analyse, strukturellen Veränderungen, Vereinbarungen und Absprachen sowie Haltung und Kultur einer Organisation" (Arbeitsstab des UBSKM (o.J.) (4)). Durch ihren Fokus auf alle am institutionellen Geschehen Beteiligte ${ }^{6}$ können auch alle diese Personengruppe von Schutzkonzepten profitieren, weshalb sich die Bundesregierung durch die Bundesinitiative nachhaltige Auswirkungen auf die Prävention sexualisierter Gewalt verspricht (vgl. ebd.). Bisher gibt es noch nicht in allen Bundesländern spezifische Informationen zum jeweiligen Vorgehen, was darin begründet ist, dass die Initiative noch nicht überall offiziell gestartet ist. Bis Ende 2018 soll dies jedoch passiert sein und sollen sich auch die noch fehlenden Bundesländer offiziell dazu geäußert haben, welche landesspezifischen Maßnahmen im Rahmen von „Schule gegen sexuelle Gewalt” ergriffen werden (vgl. ohne Autor (3) (2016)).

\footnotetext{
${ }^{6}$ Im Falle von Schulen also Schüler*innen, Eltern sowie das gesamte Kollegium der Schule.
} 


\section{Methodik der empirischen Untersuchung}

In den folgenden Kapiteln und Unterkapiteln wird die Methodik der in dieser Arbeit erfolgten Untersuchung dargelegt. Nachdem die Methodenauswahl zunächst kurz begründet wird, erfolgt die ausführliche (äußere) Betrachtung des vorliegenden Datenmaterials. Mit einem Einblick in die intensive forschungsethische Reflexion wird weiterhin dargelegt, dass das Forschungsvorhaben sich den forschungsethischen Ansprüchen dieses sensiblen Themas bewusst ist und sie entsprechend berücksichtigt. Die detaillierte Beschreibung der gewählten Methode mündet letztlich in die Vorstellung des erarbeiteten Kategoriensystems, welches die Grundlage der tatsächlichen Auswertung der Untersuchungsergebnisse darstellt.

\subsection{Begründung der qualitativen methodischen Vorgehensweise}

Für die Auswertung der Lehrer*inneninterviews wird nach Mayrings qualitativer Inhaltsanalyse vorgegangen (vgl. Kapitel 5.5). Die Wahl der Auswertungsmethode orientiert sich am bisherigen Vorgehen innerhalb des Gesamtforschungsvorhabens (vgl. Kapitel 1.5). Da auch die sonstigen Interviews mittels der Methode Mayrings ausgewertet wurden, kommt sie auch in dieser Masterthesis zur Anwendung. Ebenfalls wird sich dem bisherigen Auswertungsprozess insofern angeschlossen, als dass das Datenmaterial mithilfe von MaxQDA codiert wird (vgl. ebd.). Durch die Nutzung gleicher qualitativen Auswertungsmethode(n) wird eine möglichst hohe Vergleichbarkeit und damit eine gute Verwertbarkeit der Ergebnisse für das Forschungsprojekt gewährleistet.

\subsection{Die Auswahl der Stichprobe und ihre Besonderheiten}

Im Forschungsvorhaben des Projekts „Schutz von Kindern und Jugendlichen vor sexueller Traumatisierung" wurden für die Generierung von Ergebnissen hinsichtlich der erklärten Forschungsziele (vgl. Kapitel 1.5) verschiedene Zielgruppen erfasst. Personen der Zielgruppen waren unter anderem pädagogische Fachkräfte. Innerhalb dieses Bereichs wurden im spezifischen Kreis Fachkräfte aus Einrichtungen der Kinder- und Jugendhilfe, konkret ambulanter Hilfen zur Erziehung, und Lehrer*innen aller Schulformen definiert. Vor der Akquise gab es keine Festlegung von engen Kriterien hinsichtlich der Stichprobenauswahl (vgl. Voß et. al 2014: 13). Im Forschungsdesign des Projekts findet sich dazu folgende Aussage: „Voraussetzung ist, dass die zu Befragenden Auskunft über eigene Erfahrungen, Einstellungen und Verhaltensweisen hinsichtlich Sexualität, Grenzverletzungen und sexualisierter Gewalt geben können“ (ebd.: 13).

Die Basis der Akquise bildete das Netzwerk des Instituts für Angewandte Sexualwissenschaften sowie die im Rahmen der Jugendsexualitätsstudie PARTNER 4 
geknüpften Kontakte zu Akteur*innen der schulischen Bildung.

Ursprünglich war geplant, eine offizielle Genehmigung der zuständigen Behörden zur Durchführung der Befragung an Schulen einzuholen. Im Rahmen der Terminplanung mit zukünftigen Teilnehmer*innen zeichnete sich dahingehend kein Bedarf ab. Die Kontaktaufnahme fand zum einen in Form von Kaltakquise, also per E-Mail oder telefonisch statt. Zum anderen erfolgte aber auch eine gezielte persönliche Ansprache über vermittelnde Personen, so zum Beispiel Mitarbeiter*innen im Projekt, Studierende der Hochschule etc. (vgl. ebd. 15f).

Zur besseren Einordnung der Stichprobe ist es notwendig, auf einige Besonderheiten hinzuweisen. Dass eine Repräsentativität nicht gegeben ist, ist alleine aufgrund der Größe der Stichprobe selbstverständlich festzuhalten. Weiterhin ist auch die Zusammensetzung der Schulformen, in welcher die interviewten Personen tätig sind, nicht gleichmäßig verteilt. So sind beispielsweise Grundschullehrkräfte gar nicht vertreten, wohingegen drei der sechs Interviews eint, dass sie mit Lehrkräften von Schulen mit Förderschwerpunkt Lernen geführt wurden (Interviews FS2, FS4, FS6). In zwei Fällen arbeiten die Lehrer*innen mit Kindern und Jugendlichen, die ihren Realschulabschluss entweder erworben haben oder diesen mit dem Besuch einer Sekundarschule anstreben (Interviews FS1 und FS3). Über Interview FS5 kann hinsichtlich der Schulform keine Aussage getroffen werden. Der Hinweis auf eine Mehrheit an befragten Lehrkräften von Schulen mit Förderschwerpunkt Lernen ist insofern von Bedeutung, als dass damit zum einen besondere Bedarfe, die die Schüler*innenschaft von Schulen mit sonderpädagogischem Förderbedarf im Bereich Lernen mit sich bringt, eine zentralere Bedeutung einnehmen (vgl. Kapitel 4.5). Weiterhin ist anzunehmen, dass die Lehrkräfte von Förderschulen aufgrund ihres Betätigungsfeldes möglicherweise über andere Qualifizierungen als Lehrkräfte an Schulen ohne spezielle Förderbedarfe verfügen. Mitunter haben sie Zusatzqualifikationen erworben oder entsprechende Lehramtsstudiengänge belegt, was sie von ihren Kolleg*innen außerhalb dieser Schulform unterscheiden könnte. Diese Aspekte führen nicht zu einer geringeren Eignung der vorliegenden Stichprobe, um die formulierten Forschungsfragen zu beantworten. Jedoch sollten die Untersuchungsergebnisse entsprechend reflektiert und auf ihre Allgemeingültigkeit hinterfragt werden.

Eine weitere Besonderheit stellt die Interviewsituation von Interview FS5 dar. Dieses Interview ist insofern von den übrigen Gesprächen zu unterscheiden, als dass es das einzige war, was mit zwei Lehrkräften stattfand (vgl. Anhang I; Transkript FS). Beide Lehrkräfte haben an ihrer Schule die Rolle der Beratungslehrkraft inne und eine entsprechende Ausbildung gemacht, weshalb sie beide von der Schulleitung gebeten wurden, am Interview teilzunehmen. Im Gegensatz zu einer intimen Gesprächssituation mit nur einer interviewten Person unterliegt ein Gespräch zu dritt einer anderen Dynamik. Gesagtes erlangt durch Kenntnis einer weiteren, der Schule angehörigen Person eine breitere beziehungsweise überhaupt eine Öffentlichkeit, 
die in vertraulichen Dialogen in der Form nicht entstehen kann, wenn die interviewende Person nicht ebenfalls Teil der Schulgemeinschaft ist. In der Auswertung sollte diesem Aspekt keine übermäßig große Bedeutung beigemessen werden. Da jedoch für dieses Interview zusätzlich kein zusammenfassendes Kurzprotokoll vorliegt, sollte die besondere Gesprächssituation dennoch mitgedacht und beachtet werden.

\subsection{Beschreibung der ausgewählten Interviews}

Die der Untersuchung zugrundeliegenden Interviews wurden im Rahmen des BMBFForschungsprojektes "Schutz von Kindern und Jugendlichen vor sexueller Traumatisierung" im Zeitraum von August 2015 bis Februar 2016 geführt (vgl. Kapitel 1.4). Die interviewenden Personen waren ausschließlich Mitarbeitende im Forschungsprojekt, darunter studentische und wissenschaftliche Hilfskräfte sowie wissenschaftliche Mitarbeiter*innen. Die leitfadengestützten Gespräche dauerten jeweils zwischen 28 Minuten und 1:27 Stunden und fanden in den Schulen statt, in denen die Lehrkräfte tätig sind.

Das gesamte, in der hiesigen Untersuchung verwendete, Datenmaterial wurde in sechs Interviews mit insgesamt sieben Lehrkräften verschiedener Schulformen erhoben. Die Akquise der Teilnehmenden wurde ebenfalls von Mitarbeitenden des Forschungsprojektes durchgeführt und konzentrierte sich auf Schulen in Sachsen, Sachsen-Anhalt und Berlin. Im Vorfeld eines jeden Interviews wurden den Interviewpartnerinnen die Hintergründe des Projektes noch einmal näher erläutert (vgl. Anhang: viiff) und eine Einwilligung zum Interview (vgl. Anhang: x) vorgelegt, aus der zum einen die Freiwilligkeit der Teilnahme ersichtlich wurde, aber auch die Möglichkeit, das Interview jederzeit unterbrechen beziehungsweise auch beenden zu können (vgl. Kapitel 1.6). Diese Einwilligungserklärung wurde in doppelter Form ausgehändigt, ein Exemplar verblieb vor Ort. In mündlicher und schriftlicher Form wurden die Interviewten darüber aufgeklärt, warum und wie die Daten erhobenen werden und in welcher Form sie gespeichert und weiterhin verwendet werden. Etwaige Fragen wurden ebenfalls beantwortet.

Im Anschluss an die Interviews verfassten die interviewenden Personen jeweils ein kurzes zusammenfassendes Protokoll zur internen Verwendung. Die Audioaufnahmen der Interviews wurden von Mitarbeitenden des Projekts mit dem Programm F4 transkribiert. Das Transkript wurde anschließend mit einem anonymisierenden Code versehen und lässt somit keine Rückschlüsse auf Institutionen oder Personen zu. Da verschiedene Mitarbeiter*innen des Projekts Interviews führten und Transkripte erstellten, finden sich sowohl in der Gesprächsführung als auch in den Transkripten geringfügige Abweichungen. Da alle Interviewer*innen an der gleichen Schulung zur Durchführung der Interviews teilgenommen haben, sind dadurch jedoch keine Auswirkungen auf die empirische Erhebung zu erwarten 
(vgl. Kapitel 1.6).

Der zum Einsatz gekommene Interviewleitfaden (vgl. Anhang: iff) wurde im Rahmen des Forschungsprojektes erarbeitet und diversen Pre-Tests unterzogen. Er war Grundlage aller im Rahmen der Erhebung durchgeführten Interviews, was eine Vergleichbarkeit der Ergebnisse zulässt (vgl. Kapitel 1.5). Bisher waren die Interviews mit den Lehrkräften kein Bestandteil der Datenauswertungen innerhalb des Forschungsprojektes. Zur Auswertung der Interviews liegen sowohl die Transkripte als auch zum Teil die angefertigten Kurzprotokolle (vorhanden für die Interviews FS1, FS2, FS3 und FS6) vor. Die Kurzprotokolle dienen lediglich zum Verständnis der Interviewsituation und der das Interview mitunter beeinflussenden Bedingungen und fassen den situativen Eindruck der interviewenden Person vom Gespräch zusammen. Sie geben außerdem zum Teil Auskunft darüber, in welchem schulischen Kontext die Interviewten zu verorten sind (Fachlehrer*innen, Schulform).

Selbstverständlich werden die Ergebnisse der Untersuchung dem Forschungsprojekt nach Abschluss der Arbeit zur Verfügung gestellt.

\subsection{Forschungsethische Reflexion zum Umgang mit den Interviews}

Das vorliegende Datenmaterial ist Ergebnis einer bereits zurückliegenden Erhebungsphase (vgl. Kapitel 1.5 und 5.3). Das bedeutet, dass keines der Interviews im Rahmen dieser Masterarbeit geführt wurde und ich in keinem Fall die interviewende Person war. Auch der verwendete Interviewleitfaden wurde ohne mein Zutun im Rahmen der Aktivitäten innerhalb des Forschungsprojektes entwickelt, getestet und eingesetzt. Bezüglich dieser Aspekte besteht eine gewisse Abhängigkeit von den forschungsethischen Prozessen, die ohne mein Beisein im Vorfeld dieser Arbeit innerhalb des Forschungsprojektes stattfanden. Anders als in den Richtlinien zur zweiten Förderphase von Forschungsvorhaben im Zusammenhang mit sexueller Gewalt gegen Kinder und Jugendliche in pädagogischen Kontexten sind in den Bestimmungen des BMBFs zur ersten Förderphase keine forschungsethischen Hinweise enthalten ${ }^{7}$. Dennoch entschied sich das Forschungsteam auch ohne explizite Aufforderung dazu, bereits im Vorfeld die Befürwortung des Forschungsvorhabens und seiner Erhebung von einer Ethikkommission einzuholen. Anlässlich dieser Absicht wurde ein umfangreiches Forschungsdesign erstellt, dessen Hauptaugenmerk auf der Betrachtung forschungsethisch relevanter Aspekte liegt (vgl. Voß et. al 2014). Auf das Einreichen des Antrages folgte

\footnotetext{
7 In der Bekanntmachung zu den Richtlinien der zweiten Förderphase wird ausdrücklich auf die Notwendigkeit eines positiven Votums einer Ethikkommission hingewiesen, wenn Forschungsvorhaben Erhebungen mit oder an Menschen vorsehen und im Rahmen der Förderlinie Förderung beantragen. Jeder Antrag muss außerdem die Beschreibung des vorgesehenen Umgangs mit forschungsethischen Aspekten enthalten (vgl. Illichmann 2017).
} 
schließlich ein positives Votum der Ethikkommission der Alice-Salomon-Hochschule Berlin, sodass mit Blick auf die Erhebung, das Erhebungsinstrument, den Umgang mit Daten sowie den Umgang mit Risiken im Rahmen des von einer forschungsethischen Handhabung ausgegangen werden kann, die den Empfehlungen der Bonner Ethikerklärung entspricht (vgl. Poelchau et. al 2015).

Wenngleich die Interviews zur Auswertung bereits vorliegen, kann auch hierbei eine konkrete forschungsethische Auseinandersetzung erfolgen. Da ich bei der Durchführung des Interviews nicht anwesend war, bin ich auf die Transkripte und deren Inhalte zur Auswertung angewiesen. Ein kontinuierlicher Reflexionsprozess des eigenen Umgangs mit den Transkripten und Inhalten der Interviews ist Voraussetzung für ein forschungsethisch angemessenes Vorgehen. Forschungsethisch relevant können dabei nach von Unger beispielsweise die Fragen danach sein, wie und wo die Daten gespeichert werden und wie im Zusammenhang mit der Vertraulichkeit eine Anonymisierung des Datenmaterials gewährleistet werden kann, sodass ein Rückschluss auf einzelne Personen nicht möglich ist (vgl. von Unger 2014: 21f). Einem Großteil der von von Unger erwähnten Fragen ist mit den Ausführungen im Projektforschungsdesign bereits Rechnung getragen. Insbesondere hinsichtlich der eigenen Subjektivität ist es für einen erfolgreichen Forschungsvorgang jedoch wichtig, in einen kritischen Reflexionsprozess einzutreten. Eine subjektive Betrachtung des vorliegenden Datenmaterials ist unvermeidbar. Hilfreich für die Selbstreflexion kann in einem ersten (aber wiederkehrenden) Schritt die Vergegenwärtigung der eigenen Positionierung im Forschungsfeld sein. Diese Frage wird bereits ausführlich in Kapitel 1.3 verhandelt und dient auch in der Auswertung der Interviews als fachliche Grundlage. Ein weiterer Aspekt bezüglich meiner eigenen Forschungsperspektive ist meine berufliche Tätigkeit in der sexualwissenschaftlichen Forschung zu Sexueller Bildung im Lehramt ${ }^{8}$. Im Rahmen der Beschäftigung habe ich bereits umfangreiche Einblicke in den aktuellen Stand der Lehramtsausbildung in Sachsen und Sachsen-Anhalt erhalten. Entsprechend beeinflusst werde ich in die Auswertung der Interviews gehen, selbst wenn ich größtmögliche Objektivität anstrebe. Auch hier ist eine selbstreflexive und vor allem kritische Auseinandersetzung mit meinem Forschungsprozess während der Interviewauswertung nötig, um zu dem von von Unger beschriebenen Punkt zu gelangen: „Den verschiedenen Zugängen ist gemein, dass sie die Subjektivität der Forschenden und den interaktiven Charakter der Forschungssituation als systematische Bestandteile des Forschungsprozesses verstehen, die nicht ignoriert, sondern qua Reflexion (...) [produktiv] genutzt werden können“ (ebd.: 23f). Geplant ist dazu eine

\footnotetext{
8 Ich bin wissenschaftliche Mitarbeiterin im BMBF-Forschungsprojekt „SeBiLe - Sexuelle Bildung für das Lehramt", im Rahmen dessen bereits diverse Recherchearbeiten zum aktuellen Stand der Lehramtsausbildung sowie Gespräche mit zuständigen Institutionen erfolgten.
} 
Diskussion von Zwischenergebnissen im Team des Forschungsprojektes, um Gedankengänge zu reflektieren und zu diskutieren.

Hinsichtlich der Vertraulichkeit und Anonymisierung ist bezüglich des geplanten Vorgehens festzuhalten, dass es keinerlei Rückschlussmöglichkeiten auf Institutionen oder Personen geben wird. Im Rahmen der forschungsethischen Reflexion spielen datenschutzrechtliche Aspekte ebenfalls eine zentrale Rolle (vgl. Poelchau et al. 2015.: 4). Für das in dieser Masterthesis angestrebte Forschungsziel wird hinsichtlich des Umgangs mit dem vorhandenen Datenmaterial folgende Vorkehrung getroffen: Im Rahmen der Auswertung erfolgt lediglich Zugriff auf bereits anonymisierte Interviewtranskripte (vgl. Kapitel 5.2.). Alle Namen von Örtlichkeiten oder Personen sind dabei bereits im Vorfeld unkenntlich gemacht worden, sodass mir nicht bekannt ist, wer die interviewten Personen waren. Weiterhin ist mit der Verwahrung der Transkripte mit großer Sorgfalt vorzugehen und ein Zugriff Dritter darauf unbedingt zu verhindern. Lediglich die Ergebnisse der Forschung werden - zum Teil unterlegt durch Zitate aus den Interviews - einer Öffentlichkeit zugänglich gemacht. Es findet keine anderweitige Verwendung der Daten statt. Gleichzeitig muss sichergestellt sein, dass bei der Präsentation der Forschungsresultate etwaige relevante Aspekte nicht aus Gründen einer verfälschenden Wirkung ausgelassen werden. Mitunter ist die Auslassung von Informationen forschungsethisch begründet, um beispielsweise durch die Veröffentlichung mögliche Schädigungen zu vermeiden. Dass Wissenschaft kritisch sein kann und soll, ist dadurch nicht aufgehoben. Der zu erforschende Themenbereich der sexualisierten Gewalt, ihrer Prävention und ihrer Relevanz im Lebensraum Schule bedarf jedoch eines besonderen Maßes an Grenzachtung, Sensibilität und bedachten Umgangs. Poelchau et. al konstituieren, dass „die Generierung neuen Wissens (...) nie über die Rechte und Interessen des Individuums gestellt werden [darf]" (ebd.: 1). Sollten sich in der Auswertung derartige Spannungsfelder ergeben, werden diese immer mit Blick auf die Bedarfe der interviewten Person(en) aufgelöst.

\subsection{Aufbau des Erhebungsinstruments}

Der für die leitfadengestützten Interviews verwendete Fragebogen (vgl. Anhang III) wurde im Rahmen des Forschungsprojektes vom wissenschaftlichen Personal entwickelt. Er ist unterteilt in sechs Teilbereiche, wobei er den interviewenden Personen insbesondere Orientierung hinsichtlich der für das Forschungsvorhaben thematisch relevanten Aspekte geben sollte: Neben einer Einleitung mit Erzählimpuls unterteilt sich der Fragebogen in die Bereiche Personen/Kultur, Sexualität/Sexuelle Bildung, Beschwerden/Grenzverletzung, Kompetenz/Qualifikation sowie zur Person/Einrichtung. 
Je nach Interviewsituation hat der in der Einleitung enthaltene Erzählimpuls die beteiligten Personen bereits in eine intensive Gesprächssituation gebracht. Die Aufforderung zu erzählen, welche Berührungspunkte es in der täglichen Arbeit mit dem Thema Sexualität gibt, bot dabei den Interviewten die Option, offen in das Thema einzusteigen und erste Schwerpunkte entsprechend ihrer persönlichen Wichtung zu setzen. Im Folgenden konnten die Interviewer*innen zunächst auf die von den Gesprächspartner*innen angebotenen Inhalte eingehen und konkretere Details erfragen. Mitunter ergab sich daraus bereits ein Austausch zu Fragen aus den Teilbereichen Personen/Kultur (in der Einrichtung), Sexualität/Sexuelle Bildung, Beschwerden/Grenzverletzung oder Kompetenzen/Qualifikationen (vgl ebd.) Während der Gesprächsführung entschied daraufhin die interviewende Person, inwieweit sie zu späterer Stelle nochmals etwaige Frageinhalte aus den einzelnen Leitfadenbereichen aufgreift.

In Gänze gab der Interviewleitfaden die Möglichkeit, ein umfassendes Bild der Einrichtung im Hinblick auf ihre Zusammensetzung (Schüler*innenschaft und Kollegium) und Angebote beziehungsweise Inhalte Sexueller Bildung, welche im schulischen Alltag auftreten, zu vermitteln. Darüber hinaus setzte er eine Vielzahl von Anknüpfungspunkten, um persönliche Haltungen der interviewten Personen zu hinterfragen und den Umgang mit (sexuellen) Grenzverletzungen innerhalb der jeweiligen Institution darzustellen. Als zusätzlichen (möglichen) Gesprächsschwerpunkt sind Informationen zu besuchten, bekannten oder als notwendig eingeschätzten Fort- und Weiterbildungsangebote zu identifizieren, für den im Leitfaden verschiedenen Fragestellungen vorgehalten waren.

Demografische Angaben und die Frage nach Ergänzungen und dem Erleben der Interviewsituation runden das Erhebungsinstrument ab.

\subsection{Vorgehen bei der Auswertung}

In den folgenden Unterkapiteln wird das Vorgehen bei der Auswertung des Datenmaterials erläutert. Zunächst wird hierfür die verwendete Methode, die qualitative Inhaltsanalyse nach Mayring, generell beschrieben und im weiteren Verlauf am Forschungsvorhaben selbst erläutert. Es folgt eine kurze Reflexion zur Anwendung der inhaltsanalytischen Gütekriterien. Schließlich folgt die Darstellung des Entwicklungsprozesses des von Mayring vorgesehenen Kategoriensystems der hiesigen Analyse und die Erläuterung der für die Auswertung der Interviews gewählten Aspekte.

\subsubsection{Anwendung der qualitativen Inhaltsanalyse nach Mayring}

Das Prinzip der Inhaltsanalyse im Allgemeinen setzt dabei an, Rückschlüsse auf nichtsprachliche Phänomene aus von Interviewpersonen Gesagtem zu ziehen. Sie ist eine 
Methode der qualitativen Sozialforschung und versucht, Gesprochenes objektiv und Schritt für Schritt, also systematisch, zu erfassen, um davonausgehend zu schlussfolgern, welche übergeordnete Bedeutung das sprachliche Material hat (vgl. Lamnek / Krell 2016: 447). Eine wichtige Voraussetzung, um dieses Ziel zu verfolgen, ist eine grundlegende Offenheit, mit der sich dem Analysematerial genähert werden soll. Insbesondere den Interpretationen, welche sich erst durch die Analyse selbst ergeben, soll entsprechend unvoreingenommen begegnet werden, um den qualitativen Forschungsprozess bestmöglich voranzubringen. Weiterhin wesentlich ist, dass mit den Untersuchungsergebnissen nicht im Vorfeld aufgestellte Hypothesen verifiziert oder falsifiziert werden sollen, sondern der Formulierung derartiger Hypothesen dienen, um darüber zu einem interpretatorischen Erkenntnisgewinn zu gelangen. (vgl. ebd. 479).

Eine Form inhaltsanalytischen Vorgehens stellt die qualitative Inhaltsanalyse nach Mayring dar, welche die Aussagen von interviewten Personen darauf untersucht, was diese direkt und wissentlich benannt haben (vgl. ebd.: 486).

$\mathrm{Zu}$ Beginn der Materialauswertung erfolgt zunächst eine umfängliche Sichtung der vorliegenden Transkripte, welche möglichst nicht von einer vorgeschalteten theoretischen Auseinandersetzung mit der Thematik gekennzeichnet sein sollte. Mit Hilfe des gewonnenen Überblicks können bereits erste Ableitungen getroffen werden, welche Kategorien sich aus dem Gesagten der Interviewpartner*innen ergeben (vgl. ebd.). Bereits an dieser Stelle wird die Inhaltsanalyse ihrem qualitativen Anspruch gerecht: „Sie ist offen für empirisch begründete Kategorien, die sich aus dem Datenmaterial ergeben“ (Lamnek / Krell 2016: 486). Lamnek und Krell beschreiben das Vorgehen als „zergliedernde Kategorien[, die] analytisch gefasst werden“ (ebd.) und Einzelfälle nicht in ihrer Ganzheit widergeben, sondern zu Merkmalsausprägungen zusammenfassen.

Der Gesamtablauf der Analyse richtet sich nach folgendem, von Mayring erstellten, Modell: 


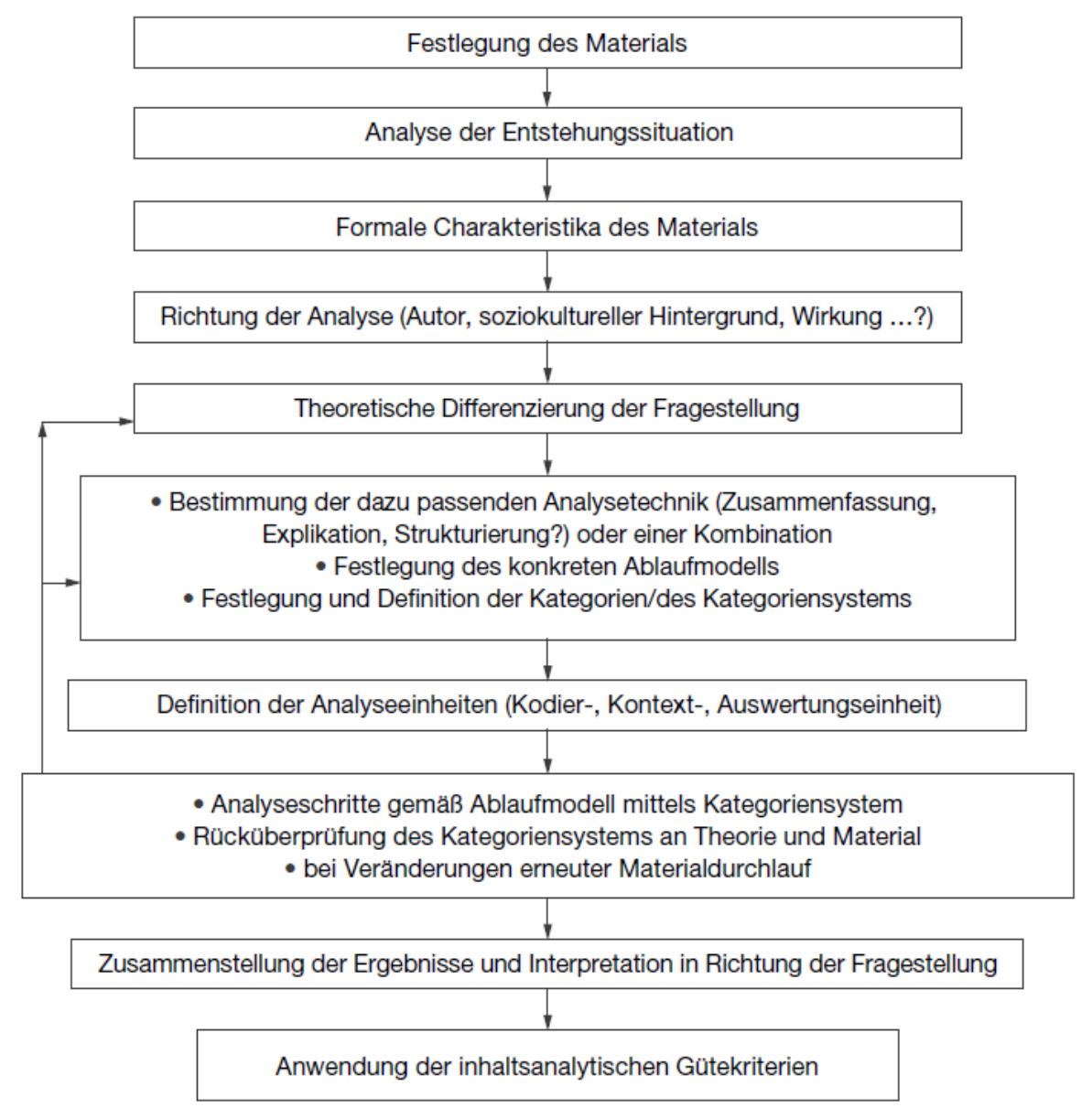

Abb. 15: Allgemeines inhaltsanalytisches Ablaufmodell

Quelle: Mayring 2015: S. 62.

Mayring betont die Wichtigkeit es systematischen Vorgehens, welches mithilfe zuvor aufgestellter Regeln der Textanalyse umsetzbar ist (vgl. Mayring 2015: 50). Das abgebildete Ablaufmodell liefert die Voraussetzungen dafür, die qualitative Inhaltsanalyse an das vorliegende Datenmaterial anzupassen und die einzuhaltenden Ablaufschritte in richtiger Reihenfolge vorzugeben.

Die Entwicklung eines Kategoriensystems auf Grundlage der vorliegenden Materialsammlung ist das wesentliche Element der inhaltsanalytischen Untersuchung nach Mayring. In Ihm werden die Untersuchungsziele konkretisiert und griffig formuliert. Zentral ist dabei die Herausforderung, die Kategorien schlüssig zu konstruieren und zu begründen. Darüber hinaus ist das Kategoriensystem und insbesondere die Tatsache, dass es nicht Ergebnis der Analyse ist, sondern als Ausgangspunkt der Untersuchung dient, ein zentraler Aspekt der Reliabilität der Analyse (vgl. ebd.: 51f; vgl. Kapitel 5.6.2).

Bei der hiesigen Untersuchung ist ein erster Schritt wie von Mayring vorgesehen - die Auswahl des Datenmaterials - nicht nötig beziehungsweise bereits im Vorfeld erfolgt, weil das auszuwertende Material bereits vorliegt und in Gänze zur Untersuchung herangezogen 
werden soll. Da die Interviews im Rahmen des BMBF-Forschungsprojektes „Schutz von Kindern und Jugendlichen vor sexueller Traumatisierung" durchgeführt wurden und bisher nicht ausgewertet werden konnten, ist es allein aus forschungsethischen Gründen angezeigt, keine zusätzlich einschränkende Auswahl innerhalb der zur Verfügung stehenden Transkripte zu treffen. Auch die Analyse der Entstehungssituation ist innerhalb des Forschungsvorhabens nur bedingt möglich, da keine Beteiligung der Autorin an der tatsächlichen Interviewsituation gegeben war. Um dennoch einen Eindruck zu gewinnen, wurden die beiliegenden Kurzprotokolle studiert (vgl. Anhang II). Zusätzlich kann an dieser Stelle noch einmal auf die vorhandene Freiwilligkeit der Teilnahme am Interview verwiesen werden, die alle teilnehmenden Intervierpartner*innen schriftlich bestätigt haben (vgl. Anhang V). Es ist ebenfalls bekannt, dass die Mitarbeiter*innen des Forschungsprojekts zur Aufzeichnung der Interviews zu vereinbarten Uhrzeiten an die Dienststellen der interviewten Personen gereist sind, weshalb davon ausgegangen wird, dass die Entstehungssituation für die Teilnehmenden unkritisch war. Auf etwaige dienstliche Störfaktoren kann nicht zurückgeschlossen werden. Da jedoch die Gesprächsführung durch das leitfadengestützte Interview sehr frei war und im Vorfeld des Interviews abermals auf Möglichkeiten des Abbruchs oder der Unterbrechung hingewiesen wurde, wurden entsprechende Vorkehrungen getroffen und Freiräume geschaffen. Leider kann hinsichtlich der kognitiven und emotionalen Verfassung der interviewenden Personen keinerlei Angaben gemacht werden, da die Interviews zum einen bereits einige Zeit zurückliegen, diese Aspekte in den zum Teil beiliegenden Kurzprotokollen nicht aufgegriffen sind und es diesbezüglich auch keine Möglichkeit gibt, mit den jeweiligen Mitarbeiter*innen in Kontakt zu treten.

Hinsichtlich der Charakteristika des Materials sind in vorherigen Kapiteln bereits die wesentlichen Merkmale zusammengetragen worden (vgl. Kapitel 5.2 - 5.4). Die zu untersuchenden Transkripte liegen der Arbeit bei und wurden auf Basis von Audiomitschnitten der einzelnen Interviews von den interviewenden Personen erstellt. Transkribiert wurde unter leichter Glättung nach den Transkriptionsregeln, auf die sich das Forschungsteam im Vorfeld der Erhebungen geeinigt hatte. Fokussiert wurde dabei auf standardorthografische Formulierungen und das Einfügen von deutlichen Pausen während des Sprechens der Beteiligten. Die Transkripte sollten außerdem in Zeilenschreibweise erstellt werden und abgebrochene Sätze mit dem Zeichen / ausweisen.

Auch die Richtung der Analyse wurde im Rahmen der Arbeit bereits an verschiedenen Stellen teilweise näher erläutert. Die forschungsethische Reflexion, aber auch die theoretischen Ausarbeitungen im Vorfeld ermöglichen ein möglichst objektives Herangehen an die Materialsammlung. Angelehnt an das von Mayring erwähnte inhaltsanalytische Kommunikationsmodell (vgl. Mayring 2015: 58f) ist festzustellen, dass Lehrkräfte verschiedener Schulformen im Rahmen des Interviews ihre persönliche Einschätzung zu 
sexualitätsbezogenen Themen und Aspekten Sexueller Bildung geben, sich zu Grenzverletzungen äußern und darüber hinaus mitteilen, welche Weiterbildungsangebote sie bereits wahrgenommen haben und welchen Fortbildungsbedarf sie bei sich im Kontext des Themenbereichs sexuelle Grenzverletzungen sehen. Die Analyse der Interviews soll die Möglichkeiten der Institution Schule identifizieren, einen Beitrag zum Schutz von Kindern und Jugendlichen vor sexualisierter Gewalt beizutragen. Sie soll weiterhin ermitteln, über welche Ressourcen Lehrkräfte diesbezüglich verfügen, in dem sie der Frage nachgeht, wie kompetent sich Lehrer*innen im Hinblick auf Sexuelle Bildung und ihrer Umsetzung im schulischen Alltag empfinden. Außerdem soll sie Rückschlüsse darauf zulassen, welchen Beitrag Aus- und Fortbildung leisten kann und muss, um unterrichtende Pädagog*innen in ihren Kompetenzen zu stärken. Die In Kapitel 2.1 aufgestellten Forschungsfragen sind hinsichtlich der Analyse des Datenmaterials richtungsweisend. Sie seien an dieser Stelle noch einmal wiederholt:

Fragestellung 1 (F1): Welchen Beitrag leistet die Institution Schule dazu, Kinder und Jugendliche durch Prävention vor sexualisierter Gewalt und/oder ihren Folgen zu schützen?

Fragestellung 2 (F2): Über welche Kompetenzen verfügen Lehrkräfte im Hinblick auf Sexuelle Bildung ihrer Einschätzung nach?

Fragestellung 3 (F3): Welche Möglichkeiten haben Lehrkräfte im Rahmen des Schulalltags, Kinder und Jugendliche in ihrer sexuellen Selbstbestimmung zu stärken?

Fragestellung 4 (F4): Welche Bestandteile der Aus- und Fortbildung von Lehrkräften befähigen sie zu einem professionellen Umgang mit dem Themenfeld Sexualität im Schulalltag?

Anknüpfend an die theoretischen Hintergründe des zu untersuchenden Gegenstandes, welche in den Kapiteln 1 bis 4 aufbereitet wurden, versprechen die oben genannten Fragestellungen F1 - F4 durch die inhaltsanalytische Untersuchung zusätzlichen Erkenntnisgewinn. Um schließlich ein umfassendes Analysemodell zu erstellen und die Analysetechnik festzulegen, erfolgt die Bestimmung von Analyseeinheiten (Kodiereinheit, Kontexteinheit und Auswertungseinheit) (vgl. Mayring 2015: 61). Maßgebliche Orientierung soll dafür das auf das zu untersuchende Material abgestimmte Kategoriensystem geben (vgl. Kapitel 5.6). Es schließt sowohl die benannten Fragestellungen als auch das vorliegende Material mit ein und wird im Laufe der Analyse kontinuierlich überprüft und ergänzt beziehungsweise überarbeitet. Mayring bietet drei Techniken an, die vorliegenden Daten zu analysieren: die Zusammenfassung, die Exploration und die Strukturierung. Im Hinblick auf die zur Verfügung stehenden Transkripte erweist sich die inhaltsanalytische Strukturierung als vielversprechend. 
Ihr Ziel ist es, „bestimmte Aspekte aus dem Material herauszufiltern, unter vorher festgelegten Ordnungskriterien einen Querschnitt durch das Material zu legen oder das Material aufgrund bestimmter Kriterien einzuschätzen“ (Mayring 2015: 67). Diese zuvor festgelegten Ordnungskriterien entsprechen den deduktiven Kategorien, die zunächst im bereits erwähnten Kategoriensystem entwickelt, also aufgrund der zuvor erarbeiteten theoretischen Ausgangslage und einer ersten Studie der Interviewtranskripte bereits vorab bestimmt werden. Vom Kategoriensystem ausgehend werden die erarbeiteten Ergebnisse in Bezug auf die Ausgangsfragen interpretiert. Letztlich folgt eine Einschätzung der Analyse entsprechend der inhaltsanalytischen Gütekriterien.

\subsubsection{Die inhaltsanalytischen Gütekriterien}

Mayring weist in der Beschreibung seiner Methode auf Wichtigkeit der Beurteilung ermittelter Ergebnisse hinsichtlich Validität, Reliabilität und Objektivität hin (vgl. Mayring 2015: 53). Eine besondere Bedeutung kommt dabei der sogenannten Intercoderreliabilität zu, die im Rahmen dieser Arbeit jedoch nicht replizierbar ist. Nach Analyse des gleichen Materials von verschiedenen untersuchenden Personen werden die Ergebnisse verglichen und eventuelle Un-Reliabilitäten erkannt, hinterfragt und versucht zu interpretieren. Ergebnis dieses Prozesses könnte beispielsweise das Aufdecken von Fehlerquellen im Untersuchungsinstrument sein und daraus folgen beispielsweise die Umgestaltung einzelner Elemente dessen (vgl. ebd.: 53f).

Der Prozess der Intercoderreliabilität lässt insbesondere Rückschlüsse auf die Objektivität zu, also darauf, ob das erhobene Ergebnis unabhängig von der untersuchenden Person ist. Dieses Vorgehen zur Prüfung des Sachverhalts ist jedoch umstritten. Nichtdestotrotz findet es in der qualitativen Sozialforschung häufig Anwendung, da es als spezifisches inhaltsanalytisches Gütekriterium gilt (vgl. ebd.: 124f).

Aufgrund der Bedingungen dieses Forschungsvorhabens können keine auf Intercoderreliabilität fokussierenden Elemente implementiert werden. Um zumindest punktuell entsprechend tätig zu werden, kommen Diskussionsrunden zum Material mit anderen Forschenden zum Einsatz.

\subsubsection{Die Entwicklung des Kategoriensystems als Grundlage der Analyse}

Wie in Kapitel 5.6.1 bereits erläutert, stellt das Kategoriensystem die Grundlage der Auswertung dar, da in ihm zunächst vorgegeben wird, worauf das vorhandene Material untersucht werden soll. Mayring erläutert, dass „alle Textbestandteile, die durch die Kategorien angesprochen werden, (...) aus dem Material systematisch extrahiert [werden]“ (Mayring 2015: 97). Dabei werden die Kategorien zunächst benannt, anschließend definiert und ein 
sogenanntes Ankerbeispiel aus dem untersuchten Text herangezogen. Wie im anhängenden Kategoriensystem erkennbar, erfolgte die Bildung von Kategorien ausgehend von den formulierten Forschungsfragen F1 - F4. Es wurden pro Forschungsfrage bis zu acht Kategorien entwickelt, die eine intensive Betrachtung verschiedener Aspekte und eine daraus folgende Beantwortung der Fragen erwarten ließen (vgl. Anhang VI). Um der forschenden Person Orientierung für eine eindeutige Zuordnung von Textbestandteilen zu geben, erfolgte die Aufstellung von sognannten Kodierregeln (vgl. Mayring 2015: 97). Sie sind ebenfalls im anhängenden Schema ersichtlich (vgl. Anhang VI).

Nach Einpflegen der Kategorien in die Analysesoftware MaxQDA wurden die Daten mit Hilfe des Programms auf dieser Grundlage mehrfach und ausgiebig untersucht ${ }^{9}$. Anschließend hätte das Schema um induktive Kategorien ergänzt werden können. Es ist sehr wahrscheinlich, dass das Material für dieses Vorgehen genügend Inhalt bereitgehalten hätte, da induktive Aspekte direkt auf dem Material selbst hervorgehen und somit nicht primär auf dem theoretischen Hintergrundwissen basieren. Sie können dabei unterstützen, die Interviews möglichst konkret abzubilden ohne von Einflüssen der forschenden Personen verzerrt zu werden. Auf Basis der Ausgangsfragestellungen würde dabei lediglich Ausschmückendes aus dem Material entfernt werden, um auf wesentliche Aussagen zu reduzieren. Zusätzlich hätte ein direkter Bezug zu einem oder mehreren Textbaustein(en) hergestellt werden können (vgl. Mayring 2015: 68ff). Nach Analyse des Materials anhand der deduktiven Kategorien wurde jedoch schnell ersichtlich, dass die Bildung von zusätzlichen induktiven Kategorien im Rahmen des Forschungsvorhabens nicht umgesetzt werden kann. Alle zusätzlich gebildeten Kategorien in die Auswertung miteinzubeziehen, wäre innerhalb des vorgesehenen Arbeitsprozesses nicht möglich gewesen, da es bereits vor der induktiven Kategorienbildung fast 30 deduktive Kategorien gab, wonach die Interviews untersucht wurden. Weiterhin ließ allein die Vielzahl der aus dem Material den deduktiven Kategorien zugeordneten Textbausteine erwarten, dass bereits auf Grundlage des zuvor aufgestellten Kategoriensystems eine differenzierte Analyse vorgenommen werden kann, wie die folgenden Abbildungen verdeutlichen:

\footnotetext{
${ }^{9}$ Wie bereits erwähnt, ist es sehr wahrscheinlich, dass sich der Aufbau des Kategoriensystems im Laufe der Analyse verändert. Unter anderem wird anhand des primär entwickelten Kategoriensystems zunächst ein Probedurchlauf am Beispiel eines zufällig ausgewählten Transkripts des Materials durchgeführt, um festzustellen, ob die benannten Kategorien tatsächlich die Inhalte des Interviews widerspiegeln. Dies ist erfolgt und die ursprünglich formulierten Kategorien wurden noch geringfügig angepasst.
} 


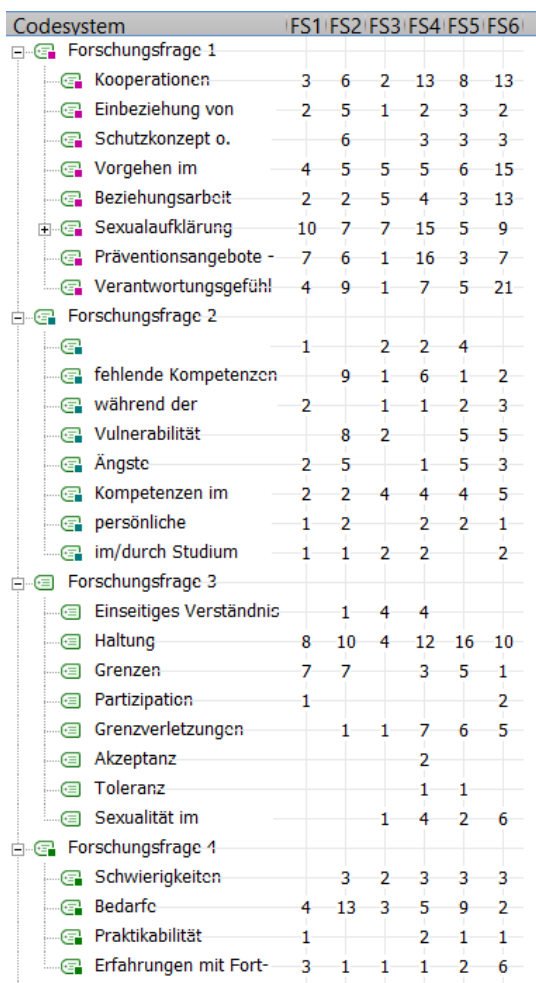

Abb. 16: Code-Matrix-Browser I

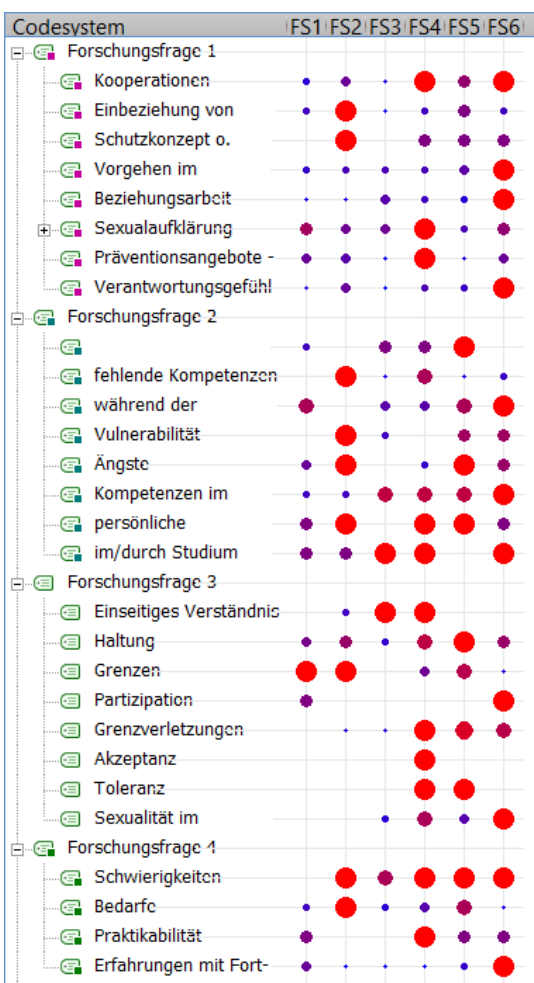

Abb. 17: Code-Matrix-Browser II

MaxQDA verfügt über die Möglichkeit, die Codings zu zählen und die ermittelte Anzahl entsprechend grafisch aufzubereiten. Die Abbildung 16 veranschaulicht deutlich, dass es eine Vielzahl an Textstellen gibt, die entsprechend der deduktiven Kategorien codiert werden konnten und eine gute Grundlage für die nachfolgende Auswertung bilden. Allen Kategorien der einzelnen Forschungsfragen können zahlreiche Aussagen der Interviews (in Abbildung 16 und 17 gekennzeichnet als FS1 - FS6) zugeordnet werden. Abbildung 17 bestätigt die Annahme, dass die vorformulierten deduktiven Kategorien tatsächlich Inhalte der Interviews aufgreifen. Bis auf wenige Ausnahmen finden sich zum Teil deutliche Häufungen von Aussagen der interviewten Personen, die Auskunft zu den aufgestellten Forschungsfragen und ihren Kategorien geben.

Aus forschungspraktischen Gründen und unter der Annahme, dass bereits die Codings entsprechend der deduktiven Kategorien eine differenzierte Auswertung ermöglichen würden, wurde deshalb im Verlauf der Analyse entschieden, auf die Bildung von induktiven Kategorien zu verzichten. Die folgende Auswertung und nachfolgende Beantwortung der Forschungsfragen stützt sich somit auf eine rein deduktive Untersuchung des vorliegenden Materials. 


\section{Auswertung der Untersuchungsergebnisse}

Im Rahmen der Auswertung wird an dieser Stelle zunächst auf die Analyse der Kategorien fokussiert, ohne die Forschungsfragen näher in den Blick zu nehmen. Stattdessen wird versucht zu erfassen, welche zentralen Aussagen von den Lehrkräften zu den einzelnen Kategorien getätigt wurden. Dabei werden zeitgleich Zusammenhänge zwischen Kategorien aufgezeigt und deutlich gemacht. Im weiteren Verlauf folgt die Übertragung dieser Auswertungsergebnisse auf die vorformulierten Forschungsfragen, um diese anschließend dezidiert beantworten zu können.

\subsection{Auswertung der Kategorien}

Die Kategorienbetrachtung und Auswertung der enthaltenen Codings erfolgt insofern nicht systematisch, als dass nicht bereits im Vorhinein mögliche Schnittmengen zwischen zwei Kategorien angenommen werden. Vielmehr wird eine Kategorie zur Auswertung herangezogen und die zugeordneten Aussagen interpretiert. Ergibt sich daraus ein offensichtlicher Zusammenhang zu einer oder mehreren weiteren Kategorie(n), werden auch jene an dieser Stelle ausgewertet und miteinander ins Verhältnis gesetzt.

Die Auswahl der betrachteten Kategorien erfolgt davon ausgehend, wie viele Codings den einzelnen Kategorien zugeordnet werden konnten. Es werden zunächst diejenigen Kategorien ausgewertet, denen besonders viele Textstellen zugeschrieben wurden. Bei diesen wird im höheren Maße eine Korrelation mit anderen Kategorien erwartet, sodass sich über diesen Weg nach und nach die meisten Kategorien erschlossen werden können. Am Ende von sehr umfangreichen Unterkapiteln (mehr als zwei Seiten) findet sich eine Zusammenfassung der interpretierten Kategorienergebnisse, um die Erkenntnisse noch einmal zu fokussieren und zu konkretisieren.

\subsubsection{Auswertung der Kategorien F1.1, F3.1 und F3.7}

Während der Auswertung der Kategorie F1.1, Sexualaufklärung, zeigt sich ein engerer Zusammenhang mit der Haltung von Lehrkräften zur Sexualität von Kindern und Jugendlichen. Es ist anzunehmen, dass die Haltung der Pädagog ${ }^{*}$ innen sich unter anderem daraus speist, wie sie Schüler*innen im Schulalltag erleben, sodass auch die Kategorie F3.1 (Sexualität im Lebensraum Schule) zur Auswertung hinzugezogen wird. Die Kategorien F1.1, F3.1 und F3.7 (Haltung) werden deshalb an dieser Stelle zusammen ausgewertet. Schon während der Codierung der Textstellen erfolgte in Kategorie F1.1 eine Unterteilung in allgemeine Aussagen zur Sexualaufklärung und Aussagen, die sich auf biologisch-medizinische in Abgrenzung zu sozialen Aspekten von Sexualität beziehen. Generell ist festzustellen, dass Inhalte der 
Sexualaufklärung in den meisten Fällen einen informierenden oder präventiven Ansatz haben. Der präventive Gedanke bezieht sich dabei primär nicht auf Formen sexualisierter Gewalt, sondern auf sexuell übertragbare Krankheiten und frühzeitige Schwangerschaften. Selbst wenn die Befragten sich zum Teil konkreter Inhalte nicht sicher waren, gaben ausnahmslos alle Interviewten Verhütung als ein zentrales Thema der schulischen Sexualaufklärung an. Anhand der Schilderungen ist anzunehmen, dass der biologisch-medizinische Betrachtung von Sexualität die größte Bedeutung zukommt. Die Lehrkräfte verweisen darauf, dass Kinder und Jugendliche in diesem Kontext ebenfalls lernen, Geschlechtsorgane und -merkmale zu bezeichnen und weiterhin Entwicklungsabläufe des menschlichen Körpers - also auch ihres eigenen - einzuordnen und zu verstehen: „Die Fortpflanzungsorgane, anatomisch, physiologisch, werden behandelt, das ist Thema, männlich weiblich, alle Prozesse die sich daran anschließen, jetzt Befruchtungsvorgang, dass man das erklären kann. Von der Eizelle befruchten, Eizelle, bis zu Geburt, die ganzen Entwicklungsstadien“ (FS1_33). Der Fokus auf der Verhinderung frühzeitiger Schwanger- beziehungsweise Elternschaft ist der Punkt, an dem sich biologisch-medizinische und soziale Perspektiven auf Sexualität mit der Haltung von Lehrkräfte zur Sexualität ihrer Schüler*innen verbinden. So berichtet eine Person: „Das ist auf jeden Fall, glaube ich, bin ich mir sicher, dass das für die Kinder extrem erstrebenswert ist, sobald sie ihre eigene Geschlechtsreife bemerken, das auch auszuleben. Also ich denke mir so, so als 13-Jährige haben Sex miteinander und brüsten sich damit, erzählen das auch. Dann natürlich diese ganzen Schwangerschaftsgeschichten, ja, die ja da auch eine Rolle spielen“ (FS6_41). Anhand des Zitats lässt sich entnehmen, dass Lehrkräfte von Schüler*innen im Lebensraum Schule ausgelebte (oder zumindest erzählte) Sexualität unter anderem als Mittel der Provokation verstehen. Insbesondere nutzen Heranwachsende ihre sexuellen Erfahrungen, um sich anderen gegenüber - zum Beispiel gegenüber den Gleichaltrigen mit weniger Erfahrungen - abzugrenzen. Wie deutlich zu erkennen, wird Sexualität dabei nicht von allen Lehrer*innen als natürlicher Bestandteil der menschlichen Entwicklung wahrgenommen, sondern zum einen als soziale Ermächtigung und zum anderen als große Gefahr hinsichtlich möglicher Konsequenzen, die es positiv zu beeinflussen gilt. Der Großteil der Lehrkräfte bringt dabei an, dass ihre Schüler*innen mitunter besonders gefährdet sind dahingehend vulnerabel zu sein und sieht dabei insbesondere die Herkunftsverhältnisse in der Verantwortung: „Also es ist eher ganz selten (...), dass die Kinder bei Mutti und Vati wohnen, also, dass das eine richtige Familie ist. Das ist eher die Ausnahme“ (FS3_21). Die Sorge vor frühzeitigen Schwangerschaften beschränkt sich dabei nicht nur auf die Sorge um die Zukunft der betroffenen Jugendlichen, sondern ist für die Lehrkräfte mehr eine bedenkliche gesellschaftliche Beobachtung, die sie nicht tatenlos hinnehmen wollen: „(...) Tendenziell denke ich, wird es immer problematischer, weil die sich auch immer mehr untereinander wiederfinden zu neuen Beziehungen, ich will gar nicht sagen Familien, weil es diese Struktur 
nicht hält, zu neuen Beziehungen und neue Elternschaften gründen und so vermischt sich nicht mehr. Es ist, es wird immer schwieriger“" (FS6_29).

Die von Schüler*innen ausgelebte Sexualität nehmen Lehrkräfte deshalb weniger als altersgerechte Entwicklung und mehr als Gefahr wahr, der sie durch die Vermittlung biologisch-medizinischer Aspekte und sozialer Details im Rahmen der schulischen Sexualaufklärung entgegen zu wirken versuchen.

\subsubsection{Auswertung der Kategorien F1.5 und F1.7}

Die Überlegung, inwieweit Lehrer*innen hinsichtlich der Sexuellen Bildung von Kindern und Jugendlichen in der Verantwortung bei sich selbst erkennen (Kategorie F1.5, Verantwortungsgefühl), nimmt in jedem Interview Raum ein.

Dass der Institution Schule im Allgemeinen dabei eine Verantwortung zukommt, wird von den befragten Personen nicht dementiert. Vielmehr wird diese Annahme durch Aussagen wie „Alle sind in der Verantwortung. Und das heißt, es ist eigentlich für jeden Klassenlehrer oder für jeden Lehrer der Schule einfach ein Thema, das wahrzunehmen und da auch darauf hinzuweisen“ (FS4_3) gestärkt und hervorgehoben. Alleine aus der Tatsache, dass „bei uns ${ }^{10}$ (...) die Kinder nun mal am meisten [sind]“ (FS2_104) ergibt sich eine Notwendigkeit dazu, Angebote zur Sexualaufklärung vorzuhalten.

Verschiedene Positionen finden sich vor allem hinsichtlich der Frage, inwieweit die interviewten Lehrkräfte selbst ein Verantwortungsgefühl hinsichtlich ihres eigenen Tuns empfinden und welche Aspekte darin eingeschlossen sind. Die Analyse der Daten zeigt auf, dass Lehrer*innen zwischen Intervention und Prävention ${ }^{11}$ und inrer individuellen Verantwortlichkeit deutlich unterscheiden. Obgleich die Lehrkräfte der Schule selbst eine Verantwortung zuschreiben zur Sexuellen Bildung von Kindern und Jugendlichen beizutragen, ist die eigene Initiative maßgeblich abhängig vom persönlichen Engagement. Eine Person stellt dazu fest, dass diese Angebote „so sporadisch [stattfinden], wie man das eben für richtig hält“" (FS6_56).

Lehrkräften in speziellen Funktionen - Fachlehrer*innen, Klassenlehrer*innen, Schulleitungen - wird jedoch grundsätzlich zunächst ein höheres Maß an Verpflichtung zugewiesen, aus ihrer Rolle heraus aufklärend einwirken zu müssen: „Also sowas machen wir schon. Aber das hängt davon ab, ob der Klassenlehrer glaubt, er sollte mit seiner Klasse sowas machen“ (FS6_47). Dass Klassenlehrer*innen tatsächlich eine besondere Position innehaben, zeigt auch die Auswertung der Kategorie F1.7, Beziehungsarbeit. Anhand des Datenmaterials wird deutlich, dass Klassenlehrer*innen zum einen bessere Möglichkeiten haben, zu den Schüler*innen ihrer

\footnotetext{
10 mit der Formulierung „bei uns“ bezieht sich die interviewte Person auf die Schule

${ }^{11}$ mit Prävention sind an dieser Stelle generell Angebote Sexueller Bildung gemeint
} 
Klasse ein Vertrauensverhältnis aufzubauen, da sie mehr Zeit mit innen verbringen. Gleichzeitig nehmen sie auch die Kinder und Jugendlichen selbst als vorrangige Ansprechpersonen wahr, denn „Kinder, die Probleme haben, (...) wenden sich erst an den Klassenleiter und der Klassenleiter vermittelt sie weiter" (F3_33). Um jedoch mit den Heranwachsenden entsprechend arbeiten zu können, konstituiert die befragte Person aus Interview FS6: „Das Entscheidende ist wirklich, dass man auch die Kinder um die dazu zu bewegen und die zu motivieren, dass die das machen, braucht man eine Basis zu denen“ (FS6_55).

Zu Verdachtsfällen von sexualisierter Gewalt führt sie weiterhin aus: „Wenn irgendein Erwachsener es schafft, dass ein Kind das Gefühl hat, hier kann ich ein Vertrauen haben, dann werden die das sagen“ (FS6_70). Die Lehrkraft stützt damit die Grundaussage anderer ebenfalls interviewter Person, die versichern, dass sie ratsuchende Schüler*innen nicht abweisen, sondern versuchen würden, sie in der Problemlösung zu unterstützen. Diese Unterstützungsmaßnahmen trennen die Befragten nicht strikt, aber dennoch klar von der konkreten Position, in der sie als Lehrer*innen möglicherweise agieren. Da das Hilfegesuch ein Zeichen von Vertrauen des*der Schülers ${ }^{*}$ in zur Lehrkraft ist, empfinden alle Interviewten eine Verantwortung, entsprechend $z u$ intervenieren. Der Umgang mit diesem Verantwortungsgefühl unterscheidet sich dabei von Interview zu Interview und reicht von verantwortlich sein, an die richtigen Personen zu verweisen (Schulsozialarbeitende, Klassenlehrer*in) bis hin zum selbst aktiv werden. Im eigenen Kollegium nehmen die interviewten Personen nicht immer ein vergleichbar großes Verantwortungsempfinden wahr, finden dafür jedoch auch Erklärungen. „Aber ich glaube das liegt eben genau daran, dass es so ein heißes Ding ist" (FS6_55). Insbesondere die eigene Unwissenheit über korrektes Vorgehen im Verdachtsfall bringt Lehrer*innen in eine schwierige Situation. Verstärkt durch die besondere Position, in der sie sich als Klassenlehrer*in befinden und die sie im Speziellen verantwortlich für das Wohlergehen der in der Klasse befindlichen Schüler*innen macht, wird die eigene Vulnerabilität von Lehrkräften deutlich: „Ich weiß nicht, ob man darauf reagieren soll. Oder ob man sich den Schüler selber nehmen soll und dann sagt 'Du, das ist eigentlich nicht schön, wenn du da zuguckst oder die Mama dir sowas erzählt. Das ist was für Erwachsene, das ist noch nichts für so 'ne Kinder wie du. Du bist zu klein /' Ich weiß nicht, wie man damit umgeht. Das ist das Problem“ (FS2_50). Zum einen empfinden sich die Lehrkräfte selbst nicht als kompetent, zum anderen erfolgt allein aufgrund ihrer Position die Zuschreibung, Vertrauensperson sein zu müssen: „Grundsätzlich sind eigentlich alle Lehrer immer auch (...) Begleiter, Berater bis hin zu Sozialarbeiter und Sozialarbeiterin. (...) Alle möglichen anderen Probleme außerhalb des Lernens. Also das heißt so dieses Ansprechpartner für alles Mögliche sein, das ist eigentlich schon immer da“ (FS4_27). 


\section{Zusammenfassung der Auswertung von Kategorie 1.5 und 1.7}

Lehrkräfte sehen die Institution Schule generell in der Verantwortung, einen Beitrag zur Sexualaufklärung von Kindern und Jugendlichen zu leisten. In Bezug auf die eigene Verantwortung unterscheiden Lehrer*innen dazwischen, Schüler*innen als Ansprechperson in Problemsituationen zur Verfügung zu stehen und der Aufgabe, aus eigenem Bestreben heraus Angebote Sexueller Bildung zu initiieren. Ersteres wird mit großer Sorgfalt verfolgt: Ratsuchende Kinder und Jugendliche werden ernst genommen und angehört. Lehrkräfte sind sich bewusst darüber, dass sie Schüler*innen ein vertrauensvolles Umfeld schaffen müssen, indem sie sich gegebenenfalls anvertrauen können. Besondere Voraussetzungen dafür werden der kontaktintensiven Klassenlehrer*in-Schüler*in-Beziehung zugeschrieben. In welcher Form die jeweilige Intervention erfolgt - ob beispielsweise in Form von Verweisung oder durch eigene Aktivität in Gesprächen mit Eltern etc. - ist maßgeblich abhängig von der persönlichen Entscheidung der Lehrkraft, da es an klaren Handlungsvorgaben fehlt. In dieser Hinsicht sind sich die Lehrkräfte auch ihrer eigenen Vulnerabilität bewusst, welche sie aufgrund von fehlendem Wissen und professionellen Handlungskompetenzen empfinden (vgl. Kapitel 6.1.6). Ein generelles Vorgehen in diesem Kontext ist dem Datenmaterial nicht zu entnehmen.

Inwieweit die Lehrer*innen den Kindern und Jugendlichen selbst Angebote Sexueller Bildung unterbreiten, ist abermals abhängig davon, für wie sehr sie es als ihre eigene Aufgabe ansehen, diesbezüglich tätig zu werden. Häufig wird diese Verpflichtung Personen in besonderer Position - wie eben beispielsweise in der Rolle der Schulleitung oder des*der Klassenlehrer*in - zugeschrieben.

\subsubsection{Auswertung der Kategorien F1.6, F1.8 und F2.5}

Die Kategorie "Kooperationspartner*innen" (F1.8) ist eine hochfrequentierte Kategorie mit 45 Codings. Sie gehört damit zu einer der Kategorie, denen die meisten Textstellen zugeordnet sind. Bei näherer Betrachtung der zugeordneten Textstellen zeigt sich, dass die Personen, mit denen die Interviewten kooperieren, zunächst in dem Schulbetrieb zugehörige Personen und in dem Schulbetrieb nicht zugehörige Personen unterschieden werden können. Es fällt auf, dass die externen Ansprechpartner*innen einer Vielzahl von Institutionen zuzuordnen sind. Zu diesen zählen beispielsweise das Jugendamt, freie Träger, die Projekte zur Sexualaufklärung anbieten (z.B. die BZgA, das DRK und die AIDS-Hilfe), das Gesundheitsamt oder Fachberatungsstellen. Zum Teil sind die Befragten unsicher, ob die genannten Institutionen tatsächlich die Anbietenden der in Anspruch genommenen Angebote waren oder ob es sich nicht doch um andere Einrichtungen handelt. Deutlich wird jedoch, dass Lehrkräfte derartige Möglichkeiten, Kinder und Jugendliche an sexuelle Themen heranzuführen, als sehr hilfreich 
erleben - zum einen ziehen die Lehrkräfte in Erwägung, dass die Schüler*innen entsprechende Angebote von externen Personen besser annehmen können, zum anderen entlastet es auch sie persönlich hinsichtlich der Aufgabe, die entsprechenden Angebote selbst vorhalten zu müssen. Ein*e Pädagog*in formuliert dazu: „Sowas würde ich mir wünschen, dass unseren Kindern auch Begrifflichkeiten beigebracht werden. Also dass der nicht vom Schwanz spricht oder so, ja. Dass das andere übernehmen, nicht wir Lehrer. Weil da haben die Kinder ja doch, ja die sehen uns da glaube ich anders. Die sehen uns da nicht (..), die sehen uns immer als Lehrer" (FS2_83).

Es zeigt sich klar, dass es kein einheitliches Vorgehen hinsichtlich der Zusammenarbeit mit Kooperationspartner*innen gibt. Weder innerhalb gleicher Klassenstufen noch Schulformen sind die in Anspruch genommenen Angebote deckungsgleich. Ebenso ist das individuelle Vorgehen der einzelnen Lehrer*innen auch innerhalb derselben Schule nicht zwangsläufig identisch, denn die Inhalte Sexueller Bildung „(...) gibt es nicht verpflichtend und das macht auch nicht jede Klasse in einem bestimmten Alter, sondern es gibt Angebote, die uns mitgeteilt werden von solchen freien Trägern oder irgendwelchen Einrichtungen“ (FS6_47). Diese können dann genutzt werden oder nicht genutzt werden, ohne, dass es diesbezügliche Vorgaben gibt. Zwar formuliert eine befragte Lehrkraft folgende Annahme: „Das, wie gesagt, das ist institutionalisiert. Ich denke, das Gesundheitsamt macht das auch in allen Schulen, da sind wir, glaube ich, keine Ausnahme“ (FS4_73), jedoch kann diese anhand des vorliegenden Materials nicht bestätigt werden.

Ein in vier von sechs Interviews erwähnter Kooperationspartner ist der Allgemeine Soziale Dienst beziehungsweise das Jugendamt. Nicht nur hinsichtlich möglicher Aufklärungsangebote wird das Jugendamt als zentraler Anlaufpunkt benannt. Es wird auch als wegweisende Institution für Verdachtsfälle sexualisierter Gewalt oder anderer kindeswohlgefährdender Umstände angesehen: „Also es gibt ja jetzt hier auch so einen relativ neuen Ordner vom Jugendamt, oder von allen möglichen Trägern der Jugendhilfe zum Thema Kindeswohlgefährdung (..) und da ist auch, sage ich mal, die Vermittlung gut gewesen“ (FS4_134). Jedoch ist auch diese Zusammenarbeit in den Schulen nicht insoweit konzeptionell festgelegt, als dass es ein einheitliches Vorgehen gäbe oder Lehrer*innen die Kooperation entsprechend der von innen eingeschätzten Bedarfe erleben. So äußert die interviewte Person in FS2 deutlich, dass sie sich wünscht, dass es „regelmäßig (...) Veranstaltungen gibt, wo man sich mit dem ASD zusammensetzt, meinetwegen alle sechs Wochen, wo konkret unsere Fälle, die die Schule betreffen, auf den Tisch kommen und wo man seine Meinung äußern kann“ (FS2_10). Als schwierig wird jedoch erlebt, dass es, wenn das Jugendamt aktiv wird und die Familie seitens des ASDs beziehungsweise einer dafür bestellten Stelle begleitet wird, keine Rückkopplung zu den Lehrerkräften gibt. So wird die Kooperation an dieser Stelle einseitig und nicht als gegenseitiger Austausch erlebt: „Ich würde mir auch wünschen, (...) dass uns 
mal jemand fragen würde: 'Was erzählt denn das Kind in der Schule? Was haben sie denn für Auffälligkeiten oder so?' Uns fragt keiner. Wir sind da wirklich außen vor“ (FS2_8).

Mehrfach geben die Befragten an, dass sie sich in konkreten Fällen Unterstützung geholt haben. An diesen Stellen gibt es eine Überschneidung von Angeboten, die außerhalb des eigenen schulischen Umfeld genutzt wurden und Angeboten innerhalb des Schulrahmens. Neben kollegialen Beratungen und Fallbesprechungen im Kollegium beziehungsweise im Austausch mit der sich regelmäßig treffenden überregionalen Beratungslehrer*innenseminargruppe gibt es eine Personengruppe, der eine außerordentlich große Bedeutung zukommt: Schulsozialarbeitende.

Die Notwendigkeit dieser Berufsgruppe an Schulen beschreibt eine interviewte Lehrkraft deutlich: „Die Institution Schule könnte solche Probleme ohne Sozialarbeiter gar nicht mehr lösen“ (FS5_L1_113). Bis auf Interview FS1, in der an der entsprechenden Schule keine Position innerhalb der Schulsozialarbeit besetzt oder vorgesehen ist, sind sie für keine der interviewten Personen wegzudenken. Alle übrigen interviewten Personen schreiben Schulsozialarbeiter*innen eine tragende Rolle im schulischen Alltag und insbesondere bei der Klärung von problematischen Situationen zu. Der Vorteil, über den Personen innerhalb der Schulsozialarbeit verfügen, ist auch gleichzeitig ihr Nachteil und wird von den Befragten uneinheitlich bewertet: Einerseits wird die Möglichkeit, über das Unterrichtsgeschehen hinaus oder mitunter auch parallel dazu für Kinder und Jugendliche ansprechbar zu sein, als großer Gewinn erlebt. Eine Lehrkraft äußert dazu, dass Schulsozialarbeiter*innen „da noch andere Möglichkeiten [haben], (...) weil die (...) auch die Kinder aus dem Unterricht rausnehmen und ein individuelles Gespräch auch führen, diese Möglichkeiten haben wir ja so gar nicht“ (FS5_L1_118). Gleichzeitig erschwert die Position, in der sich die sozialpädagogischen Fachkräfte befinden auch den Prozess, dass Schüler*innen sie als Ansprechperson erkennen und letztlich auch nutzen, denn „ein Schulsozialarbeiter wird nicht zur Vertrauensperson, weil er diese Position innehat, auch wenn sie noch so nett und freundlich ist" (FS6_70). Nichtdestotrotz sind Schulsozialarbeitende für die interviewten Lehrer*innen die Personengruppe, mit der sie - übereinstimmend - am ehesten kooperieren. Mitunter werden sie ganz selbstverständlich am Problemlösungsprozess beteiligt („Dann ziehe ich mir meist den Schulsozialarbeiter auch mit dazu." (FS4_33)) oder als diejenigen eingespannt, die nach der Vorarbeit durch die Lehrkraft das für die spezifische Situation Notwendige in die Hand nehmen („Das Individuelle macht dann zum Beispiel die Frau **** als Sozialarbeiterin.“ (FS5_L1_108)).

Eng verwoben mit der Frage, mit wem Lehrkräfte selbst kooperieren und inwieweit es auch Unterstützungsmöglichkeiten innerhalb der eigenen Schule gibt, ist die Kategorie F2.5, welche Kompetenzen im Lehrer*innenkollegium beschreibt. Die Wahrnehmung dieser Kompetenzen steht im deutlichen Gegensatz dazu, als wie hilfreich und notwendig Schulsozialarbeitende 
erlebt werden. Nur vereinzelt gibt es eine*n Fachlehrer*in, welche ${ }^{*} r$ hinsichtlich der Verhandlung sexueller Themen kompetent und engagiert erlebt wird. Zumeist wird den Kolleg*innen jedoch ebenso wenig diesbezügliche professionelle Kompetenz zugeschrieben, wie die befragten Personen bei sich selbst wahrnehmen, wie folgende Aussage verdeutlicht: „(...) Für die allermeisten ist es einfach so, naja, aus dem Bauch heraus“ (FS4_124). Im Gesamtkollegium der Schule sind es einheitlich nur Schulsozialarbeiter*innen, die als Kooperationspartner hinsichtlich Angebote Sexueller Bildung identifiziert werden.

Überschneidungen bezüglich der beiden in diesem Kapitel bereits dargestellten Kategorien weisen die Codings zu Kategorie 1.6, Einbeziehung von Bezugspersonen, auf. Auch hinsichtlich von relevanten Bezugspersonen, die in Problemsituationen hinzugezogen werden, kann zwischen externen und internen Personengruppen unterschieden werden. Deutlich wird, dass die innerhalb der eigenen Schule hinzugezogenen Fachkräfte insbesondere Personen sind, die für die Lehrkräfte selbst Bezugspersonen darstellen und nicht vorrangig für die Kinder und Jugendlichen. Im hierarchischen Gefüge der Schule wird zunächst die Schulleitung involviert, insbesondere, wenn es um mögliche Verhaltenssanktionierungen geht, wie die befragte Lehrkraft in FS5 berichtet: „Das geht dann auch ganz schnell zum Schulleiter (...). Und das ist ein ganz gutes Zusammenwirken mit der Schulleitung, die also da auch nicht lange wartet, die also sofort Gesprächsbereitschaft signalisiert und wenn's in der Pause ist“ (FS5_L2_51). Weiterhin sind es abermals die Schulsozialarbeiter*innen, die die Lehrkräfte selbst als Bezugspersonen einordnen.

Externen Bezugspersonen, die je nach Situation in die Schule geladen und beteiligt werden, sind in allen Fällen, die in den Interviews beschrieben werden, die Eltern beziehungsweise Erziehungsberechtigten. Die Einbeziehung von Eltern in bestimmten Kontexten beschreiben die befragten Lehrkräfte zum einen als notwendig, da die meisten Schüler*innen noch minderjährig sind. Dennoch fällt es den Pädagog*innen mitunter nicht leicht, sich für die Involvierung von Eltern(teilen) zu entscheiden, was verschiedene Gründe hat. Der ${ }^{\star}$ die Befragte des Interviews FS4 führt dazu aus: „Wenn Schüler kommen mit Problemen, die das Elternhaus betreffen, also so aus dem häuslichen Bereich, dann ziehe ich mir meist den Schulsozialarbeiter auch mit dazu (...) um dann/ also mit dem Schüler, der das Problem hat, der muss dann natürlich auch bereit sein, dass das noch jemand anderes erfährt, aber in der Regel ist das auch so. Naja, und dann muss man halt gucken, wie man weiter verfährt. Das ist dann immer ein bisschen schwieriger. Ob man da jetzt offensiv agiert und die Eltern einlädt oder es ein Fall, wo man sagt, da muss man jetzt ans Jugendamt oder/ also das ist dann schon sehr unterschiedlich“ (FS4_33). Hier wird deutlich, was auch in anderen Interviews zur Sprache kommt: Eltern werden nicht immer als verlässliche Bezugspersonen erlebt, sodass ihre Einbeziehung durchaus Schwierigkeiten birgt. Als besonders problematisch schätzen die Befragten ein, dass sie keine Handhabe haben, wenn Eltern nicht mit innen 
zusammenarbeiten wollen oder können oder in der Zusammenarbeit nicht ehrlich sind. An dieser Stelle sind den Lehrer*innen die Hände gebunden, denn: „Wenn ich jetzt die Mutter aber einlade (.), dreimal kommt sie nicht, und beim vierten Mal sagt die 'So sprechen wir zu Hause nicht, solche Wörter hört der nicht bei uns.' Und dann wissen wir nicht weiter“ (FS2_37).

\section{Zusammenfassung der Auswertung von Kategorie F1.6, F1.8 und F2.5}

Zur Bearbeitung von Themen Sexueller Bildung oder problematischen Vorkommnissen im Kontext von Sexualität (zum Beispiel im Verdachtsfall von sexualisierter Gewalt) kooperieren Lehrkräfte mit Personen innerhalb des Schulbetriebs und externen Fachkräften. Diese Kooperationen sind nicht institutionell strukturell festgelegt, sondern werden durch das individuelle Vorgehen der Lehrkraft entschieden. Um den Schüler*innen Angebote Sexueller Bildung zu unterbreiten, erfolgt häufig die Inanspruchnahme von Diensten freier Träger. Die Lehrkräfte schätzen ein, dass Mitarbeitende der freien Träger besser geeignet sind, mit den Kindern und Jugendlichen zu Themen von Sexualität zu arbeiten, da sie innen nicht so nahestehen.

Als weitere zentrale Kooperationspartner*innen werden das Jugendamt/der Allgemeine Soziale Dienst sowie die in der Schule ansässigen Schulsozialarbeitenden erlebt. Innerhalb des Lehrer*innenkollegiums berichteten die Befragten nur von vereinzelten Kompetenzen bei beispielsweise Fachlehrer*innen, dann jedoch zu themenspezifischen Punkten (zum Beispiel biologische Aspekte). Darüber hinaus werden weitere Personen involviert, wenn ihre Einbeziehung angezeigt ist. Dies können beispielsweise die Schulleitung oder Eltern sein, also Personen, die entweder von den Lehrkräften selbst als Bezugspersonen erlebt werden oder dies für die Schüler*innen darstellen. Insbesondere die Zusammenarbeit mit Eltern beschreiben die Befragten als problematisch, da sie keine Handhabe haben, sie zu Ehrlichkeit oder generell zur Zusammenarbeit anzuhalten.

\subsubsection{Auswertung der Kategorie F3.4}

In mehreren Interviews berichten Lehrkräfte über sexuelle Grenzverletzungen, die sie im Schulalltag wahrnehmen (Kategorie F3.4). Diese Textpassagen sollen in diesem Unterkapitel gesondert betrachtet und ausgewertet werden.

Während der Sichtung der zugeordneten Codings fällt schnell auf, dass verschiedene interviewte Personen ein sich ähnelndes Phänomen beschreiben. Bestandteil dessen sind zum einen Kinder und Jugendliche, die sich Mitschüler*innen gegenüber sexualisiert verhalten, ohne gezielt übergriffig sein zu wollen. So beschreibt eine Lehrkraft diesen Aspekt folgendermaßen: „Ich nehme das wohl war, dass es Klassen gibt, wo so ein sehr rüder 
Umgangston herrscht und dass die Jungs es im Grunde auch für fast normal halten, sexualisierte Anspielungen irgendwie zu machen“ (F4_53). Den Gegenpart - und die Kombination beider Teilphänomene mag das eigentlich Problematische sein - bilden Heranwachsende, die aus Sicht der Lehrkraft sexuelle Grenzverletzungen mitunter nicht als das wahrnehmen, was sie sind: „Und das ist insofern ein großes Thema und eben auch eine große Gefahr, dass sie, in welcher Form auch immer, Opfer von Grenzverletzungen werden, die sie oft auch, also zumindest nehme ich das so wahr, gar nicht so registrieren“ (F4_3). Auffällig ist, dass alle sich zu sexuellen Grenzverletzungen äußernden Personen auch sexualisierte verbale Äußerungen klar in den potentiell übergriffigen Bereich einschließen. Jedoch ist es genau dieser Punkt - die verbalen Übergriffe - an dem die Schüler*innen selbst offenbar ein anderes Maß ansetzen als die Pädagog*innen: „Also was verbale Grenzverletzung betrifft, sowieso. Also das ist für manche so normal, das nehmen die gar nicht wahr“ (FS4_106). Im Gegensatz zu den Heranwachsenden sind Lehrer*innen augenscheinlich sehr sensibel gegenüber derartigen Grenzverletzungen und benennen diesen als kontinuierlich auftretenden Faktor im schulischen Alltag, der mitunter auch vor ihnen selbst nicht Halt macht: „Ja, das gibt eine sehr sexualisierte Sprache. Auch uns gegenüber. Also ich bin schon öfter mal die alte Fotze und das Tittenmonster und so" (F6_41). Deutlich wird weiterhin, dass die Art der Intervention und ihr Ausmaß nicht nur von der Beschaffenheit des übergriffigen Verhaltens ausgeht, sondern das gesamte Setting in die Bewertung der Gesamtsituation durch die Lehrkraft einfließt. Handelt es sich bei der (verbal) übergriffigen Person um ein ${ }^{*}$ Schüler*in ${ }^{12}$, der die sonst nicht auffällig ist oder fanden beziehungsweise finden die (verbalen) Übergriffe in einem schulexternen Kontext (zum Beispiel über soziale Netzwerke) statt, ist das Maß an Intervention durch die Lehrkraft weniger eindringlich als in anderen Fällen. So ist für die interviewten Lehrkräfte eine drastische Maßnahme vor allem immer dann angezeigt, sobald die Grenze von verbalen zu offensichtlich körperlichen Grenzverletzungen überschritten wird: „Es war jetzt keine Situation im Unterricht, wo ich jetzt sagen muss, oh, ich muss jetzt dazwischen springen, weil die die angrabschen oder sowas, das nicht. Das sind meistens irgendwelche Sachen, die erzählt werden oder die in Facebook geschrieben werden“ (F6_45).

\footnotetext{
12 übrigens werden in allen Fällen Jungs als die übergriffigen Personen benannt und Mädchen (und nur selten Jungs) als diejenigen, an die sich die unbewussten oder bewussten Angriffe richten
} 


\subsubsection{Auswertung der Kategorien F1.2, F1.3 und F1.4}

Die Aussagen zu Schutzkonzepten ${ }^{13}$ und konkreten Handlungsleitfäden bei Verdachtsfällen (Kategorie F1.3) sind in den Interviews zum einen eng verwoben mit Aussagen zum Vorgehen bei Verdachtsfällen im Allgemeinen (F1.4). Zum anderen knüpfen sie eng an den Informationen an, die die Befragten zu generellen Präventionsangeboten an ihrer jeweiligen Schule übermitteln (F1.2). Den Grund für diesen engen Bezug hat die Analyse des Datenmaterials zeitnah ergeben: An den Schulen der befragten Personen gibt es keine Schutzkonzepte im engeren Sinne. So berichten die Lehrkräfte in drei Interviews, dass es an ihrer Schule keinerlei konkrete oder sich wiederholende Angebote oder Projekte zur Prävention sexualisierter Gewalt gibt. Zwei Lehrer*innen ergänzen im weiteren Verlauf, dass sie mit ihrer Klasse bereits einen entsprechenden Film geschaut haben beziehungsweise sie mündlich auffordern, fremden Personen gegenüber misstrauisch zu sein. Jedoch sind dies die einzigen ergriffenen präventiven Maßnahmen, wie die Lehrkraft in Interview FS2 aufzeigt: „Wo dann sofort wir wieder belehrt haben (...): 'Ihr geht nicht mit Fremden mit. Wenn euch jemand anspricht, ruft ganz laut um Hilfe und so.' (...) Also das wissen sie, zumindest sagen wir ihnen das." (FS2_87).

Zwei weitere Lehrer*innen berichten davon, dass es an ihrer Schule ein Präventionskonzept im weiteren Sinne gibt, die auch die Prävention von beziehungsweise Intervention bei sexualisierter Gewalt einbezieht: „Also dieser Bereich, das haben wir so in ein Konzept gebracht, wo wir versucht haben, eben auch so eine Struktur reinzubringen. Also dass es jetzt nicht beliebig ist und jeder Lehrer irgendwie, irgendwo, irgendwas macht, sondern dass das schon so ein bisschen einem Plan auch folgt“" (FS4_81). Inwiefern aus diesen Konzepten tatsächlich ein strukturell angelegtes Vorgehen resultiert, ist den Interviews nicht zu entnehmen. Der Großteil des untersuchten Materials lässt den Rückschluss zu, dass es an den Schulen kaum gezieltes Vorgehen gegen sexualisierte Gewalt gibt. Insbesondere findet keine grundsätzliche Auseinandersetzung mit der Thematik im Sinne des vom UBSKM forcierten Schutzkonzeptes statt, was auch Risiken in der Schule selbst in den Blick nehmen würde. Sowohl die vorgehaltenen Angebote zur Prävention sexualisierter Gewalt als auch das Vorgehen im Verdachtsfall sind überwiegend gänzlich abhängig von der individuellen Entscheidung der involvierten Lehrkraft oder entsprechen dem üblichen, aber nicht geregeltem

\footnotetext{
${ }^{13}$ An dieser Stelle sind Schutzkonzepte konkret nach dem Verständnis des UBSKMs gemeint, also professionelle Konzepte mit festgelegten Bestandteilen wie einem entsprechenden Leitbild, einem Interventionsplan, festgelegten Kooperationspartner*innen und Verantwortlichkeiten, Richtlinien für Fortbildungen, einem für alle in der Schule Anwesenden geltendem Verhaltenskodex, Partizipationsund Beschwerdemöglichkeiten für Kinder und Jugendlichen sowie vorgehaltener Präventionsangebote (vgl. Rörig 0.J.)
} 
oder schriftlich festgehaltenem Vorgehen innerhalb der spezifischen Schule. Auch die Quantität der Präventionsangebote ist nicht schulintern oder gar schul(form)übergreifend geregelt, wie die Antwort einer befragten Lehrkraft darauf offenbart, wer und wie häufig derartige Angebote unterbreitet: „Einmal, höchstens zweimal im Jahr und auch nur die Leute, die denken, wir sollten es tun“ (FS6_50).

Eine solche übliche, wenngleich auch nicht generell vorgeschriebene Maßnahme ist es, die Schulleitung über die Vorfälle beziehungsweise Verdachtsfälle in Kenntnis zu setzen. In den geführten Interviews zeigt sich die Schulleitung in einer Position, die von den Lehrkräften dann miteinbezogen wird, wenn das weitere Vorgehen unklar ist oder von hierarchisch höherer Ebene entschieden werden sollte: „Und die nächste Instanz bei uns ist die Schulleitung auf jeden Fall. Und dann hat die Schulleitung sozusagen ihr Prozedere wie es von dort aus weitergeht“ (FS6_68). Die Involvierung des*der Schulleiter*in oder sonstiger Dritter geht für die befragten Lehrkräfte mit einer zusätzlichen Schwierigkeit einher: „(...) de[r] Schüler, der das Problem hat, der muss dann natürlich auch bereit sein, dass das noch jemand anderes erfährt]“ (FS4_33). An dieser Stelle wird das professionelle Handeln aufgrund der fehlenden konzeptionell festgehaltenen Vorgehensweise durch die persönliche Beziehung zu den betroffenen Kindern beeinflusst oder sogar gehemmt. Da den Lehrer*innen zum einen die Beziehung zu den Schüler*innen wichtig ist (vgl. Kapitel 6.1.2), sie sich gleichzeitig aber auch verantwortlich fühlen, sich für deren Wohlergehen einzusetzen (vgl. ebd.) kommt es dann zu einer schwer lösbaren Situation, wenn Betroffene die Involvierung weiterer Personen nicht wünschen. Durch die fehlende klare Regelung der Abläufe fühlen sich die Befragten mitunter von diesen Situationen überfordert: „(...) Wir wissen nicht, wo wir damit hingehen sollen“ (FS2_6). Mehrfach berichten die Interviewten dann davon, dass sie selbst mit den betroffenen Kindern und Jugendlichen externe Anlaufstellen aufgesucht haben.

Bis auf eine befragte Person, die konkret einen „relativ neuen Ordner vom Jugendamt, oder von allen möglichen Trägern der Jugendhilfe zum Thema Kindeswohlgefährdung“ (FS4_134) anspricht, erwähnt niemand vorgegebene Handlungsabläufe im Verdachtsfall. Inwieweit der Ordner des Jugendamts dahingehend Hilfestellung gibt, wird ebenfalls nicht erläutert. Ein weiterer Aspekt, der im Rahmen der Auswertung auffällt, ist die Tatsache, dass präventive Angebote im Kontext sexualisierter Gewalt - so sie denn an der jeweiligen Schule vorhanden sind - vorrangig darauf zielen, Schüler*innen zu befähigen, drohende Grenzverletzungen überhaupt als solche zu identifizieren. Mehrere befragte Personen bekräftigen, dass es wichtig sei, dass Kinder und Jugendliche lernen, Grenzverletzungen wahrzunehmen und in dieser Situation handlungsfähig zu sein. Gleichzeitig wird durch die Materialanalyse auch deutlich, dass Lehrkräfte diese Notwendigkeit weniger als konkrete Maßnahme gegen sexualisierte Gewalt verstehen. Viel mehr ist die Prävention der sexualisierten Gewalt ein Aspekt innerhalb der Persönlichkeitsentwicklung der Schüler*innen, auf den die Schule kontinuierlich Einfluss 
nehmen sollte: „Diese Stärkung einfach der Mädchen und Jungen, dass sie überhaupt erstmal in der Lage sind, im Falle von Grenzverletzung einfach auch irgendein Handlungsrepertoire zu haben. Das ist für mich eigentlich der ganz zentrale Punkt, weil ich denke, das reicht eigentlich nicht, wenn man dazu jetzt immer mal ein Projekt macht oder so, sondern das ist eigentlich was, was sich durchziehen muss“ (FS4_140).

Wie Schulen mit sexualisierten Übergriffen innerhalb des eigenen Hauses umgehen, wird ebenfalls thematisch in den Interviews verhandelt. Auffällig ist, dass Lehrer*innen diese Vorfälle nicht unmittelbar mit der Thematik der sexualisierten Gewalt verknüpfen. Zumeist greift auch an dieser Stelle die Abhängigkeit von des*der individuell involvierten Lehrer*in und seine*ihre Bewertung der Situation. So ist auch das weitere Vorgehen der beteiligten Lehrkraft wesentlich abhängig davon, welche Personen betroffen oder auffällig sind, wie sich dem ausgewerteten Material entnehmen lässt: „Wenn ich weiß, dass ein Schüler, der mir sonst nicht in der Richtung jemals aufgefallen ist, plötzlich mit so 'nem Blödsinn anfangen sollte, dann kann man das unter vier Augen schon klären, dass er damit aufhört“" (FS5_L2_69). Hingegen reicht einer anderen befragten Person bereits ein „blöder Blick, um klar an[zuweisen], dass derartige Handlungen nicht mehr stattfinden. (...) Ansonsten hat das sofort Konsequenzen mit weiterführenden Maßnahmen“ (FS5_51). Eine Einheitlichkeit im Vorgehen ist auch hier nicht erkennbar.

\section{Zusammenfassung der Auswertung von Kategorie F1.2, F1.3 und F1.4}

An den Schulen der befragten Lehrer*innen gibt es keine Schutzkonzepte nach dem Verständnis des UBSKM. Bis auf wenige Ausnahmen ist das Vorgehen im Verdachtsfall daher genauso wenig institutionell geregelt wie das Vorhalten von Präventionsangeboten im Kontext sexualisierter Gewalt. Beides ist in Vorgehensweise, Ausgestaltung und Quantität nahezu immer abhängig von der beteiligten Lehrkraft und steht mitunter auch in Abhängigkeit zu anderen beteiligten Personen (zum Beispiel den betroffenen oder übergriffigen Schüler $\left.{ }^{\star} i n n e n\right)$. Das bedeutet, dass nicht immer in vergleichbaren Situationen auch ähnliche Maßnahmen ergriffen werden und nicht sichergestellt ist, dass die umgesetzten Maßnahmen wirksam sind. Die Entscheidung steht in engem Zusammenhang zur Situationsbewertung durch den*die involvierte ${ }^{*} n$ Lehrer*in. Die Lehrer*innen sind davon teilweise überfordert und erleben sich insbesondere hinsichtlich der Vertrauensbeziehung zur betroffenen Person in einem Zwiespalt, wenn es um die Einbeziehung Dritter geht. Erste Ansprechpersonen in derartigen Situationen stellen häufig die Schulleitungen dar, welche in der Wahrnehmung der Befragten über weiteres Handlungsrepertoire verfügen.

Angebotene Präventionsmaßnahmen zielen zumeist auf die Wahrnehmung der eigenen Bedürfnisse und Grenzen ab und auf das Erlernen, dies zu artikulieren. 


\subsubsection{Auswertung der Kategorien F2.1, F2.4, F2.7 und F2.8}

In der Auswertung zeigte sich eine Korrelation zwischen den Kategorien im/durch das Studium erworbene Kompetenzen (F2.1), Ängste (F2.7), Vulnerabilität (F2.8) und fehlenden Kompetenzen der Lehrkräfte (F2.4), sodass diese im Folgenden gemeinschaftlich betrachtet werden. Dabei ist zunächst festzustellen, dass die fehlenden Kompetenzen, die Lehrer*innen bei sich selbst erkennen, sehr weitreichend sind. So signalisieren die befragten Personen konkret fehlendes Faktenwissen („Aber es gibt doch sicher auch Frauen, die sexuelle Gewalt ausüben? Wo fängt es an, wo hört es auf? Da mal, das weiß glaube ich keine von uns.“ (F2_20)), aber gleichzeitig zum Teil auch Unkenntnis darüber, an wen sie sich wenden könnten, um dennoch handlungsfähig zu sein („Für unsere Kleinen wüsste ich nicht, an wen ich mich jetzt wenden könnte, wer das mit denen machen würde. Habe ich absolut keine Ahnung.“ (FS2_41)). Als Ursache dafür benennen die Pädagog*innen die Problematik der fehlenden Inhalte zum Thema im Lehramtsstudium: „Ich denke, da kann ich auch für die meisten anderen sprechen, es gibt keinen Punkt in unserer Ausbildung als Lehrer, wo das eine Rolle spielt“ (FS4_124). Sie knüpfen damit eng an die bereits dargelegten theoretischen Annahmen an und bestätigen abermals, dass sie um die Notwendigkeit der Vermittlung von Inhalten Sexueller Bildung für zukünftige Lehrer*innen (vgl. Kapitel 3.4) wissen und diese einfordern: „Ich fände es wichtig, dass, das thematisch in die Lehrerausbildung reinkommt“ (FS6_132). Eine Lehrperson gibt an, sich im Studium (der Förderpädagogik) an verschiedenen Punkten mit der Thematik Sexualität beziehungsweise sexualisierte Gewalt beschäftigt zu haben. Neben einer weiteren Lehrkraft, die eine Vorlesung zur Gesundheitserziehung besucht zu haben, in welcher das Phänomen der sexualisierten Gewalt randständig thematisiert wurde, hat keine der sonstigen befragten Personen entsprechende Inhalte in ihrer Ausbildung bearbeitet. Aus Sicht der Lehrkräfte mündet das fehlende Basiswissen in der Schulpraxis in fehlende Handlungskompetenz, insbesondere in Verdachtsfällen sexualisierter Gewalt. So offenbart ein*e Pädagog*in: „Ich weiß nicht, wie man damit umgeht. Das ist das Problem“ (FS2_50). Im Umgang mit möglicherweise betroffenen Kindern und Jugendlichen sind die Befragten über den genauen Ablauf sehr unsicher, was sich innerhalb des Datenmaterials an verschiedenen Stellen eruieren lässt („Soll ich mir alles erzählen lassen, soll ich die Eltern mit einbeziehen?" (FS3_91)). Diese Aussagen führen zu dem Schluss, dass es in den Schulen keinen konzeptionellen Handlungsrahmen gibt, an dem sich Lehrkräfte in Verdachtsfällen orientieren können. Zum Teil gelingt es ihnen dann, sich Beistand innerhalb der eigenen Schule oder von externen Stellen zu holen (vgl. Kapitel 6.1.3). Jedoch erleben Lehrer*innen auch diese Momente als mitunter problematisch: „Wir haben da auch mit verschiedenen Stellen keine positiven Erfahrungen gemacht (...). Deshalb nimmt man sich da glaube ich auch immer ein Stück zurück. Wir sagen uns, uns wird nicht weitergeholfen, Hilfen fehlen uns“ (FS2_4). Der fehlende institutionelle Rahmen führt zwangsläufig zu einem hochgradig 
individualisierten Handeln (müssen) der beteiligten Lehrer*innen und verhindert ein standardisiertes Vorgehen im Verdachtsfall. Es ist weiterhin anzunehmen, dass die als ungenügend erlebte Unterstützung dazu beiträgt, dass pädagogisch Tätige im Lehrbetrieb gehemmt sind in potentiellen Verdachtsfällen einzugreifen beziehungsweise sie Ängste haben, mit der Situation nicht kompetent umgehen zu können. Dass auch innerhalb der eigenen Schule nicht immer fachkundige Begleitung zur Verfügung steht (vgl. Kapitel 6.1.3), trägt zur Verunsicherung der Lehrkräfte bei, aber auch zu ihrer Vulnerabilität. Hinzukommt die Sorge, durch Fehleinschätzungen oder fehlerhafte Handlungen, also der Unwissenheit über das angemessene Vorgehen, selbst rechtlich belangt werden zu können: „Wo mache ich mich denn strafbar, wenn ich das Kind dann Näheres gefragt habe oder so? So 'ne Sachen, die kennen wir ja überhaupt nicht“ (FS2_27). Auch die hohe psychische Belastung, die mit Verdachtsfällen sexualisierter Gewalt auf die in Kenntnis gesetzten Lehrkräfte einwirkt, thematisieren die Befragten. Gleichzeitig stellen sie dar, dass es auch innerhalb des eigenen Hauses keine adäquaten Möglichkeiten zur Aufarbeitung derartiger Belastungen gibt. Eine Person äußert sich dazu wie folgt: „Mein Hauptthema ist, dass wir überhaupt keine Form von Supervision bekommen. Überhaupt nicht. Und das ist eigentlich superkrass. Also einerseits sollen wir sozusagen die vertrauenswürdigen Erwachsenen sein, die zum Teil extrem involviert sind in das Ganze. Manchmal mehr als die, die es wirklich professionell betreiben. Und andererseits gibt es überhaupt nichts, was mein Befinden bei der ganzen Sache abfängt“ (FS6_100). Insbesondere das Empfinden (oder die Tatsache?), dass Lehrer*innen nahezu immer in ihrer beruflichen Tätigkeit auf sich allein und die persönlichen Kompetenzen angewiesen sind, führt zur Verstärkung der individuellen Verletzbarkeit: „Wir fühlen uns schon, also ich, fühle mich sehr alleine gelassen. (...) Ich würde mir öfter Hilfe wünschen“ (FS2_8). Durch die fehlenden konzeptionellen Rahmungen ist es Lehrer*innen gibt es keine klaren Handlungsanweisungen, die Lehrer*innen entlasten und sich sicher fühlen lassen. Das bedeutet, dass sie keine Möglichkeit haben, die ihnen anvertrauten Informationen von dieser Vertrauensebene in einen strukturellen Rahmen zu überführen. Stattdessen müssen ihre Entscheidungen überwiegend individualisiert getroffen werden ohne in nachbereitenden Settings, wie beispielsweise Supervisionen, aufgearbeitet zu werden. Dies unterstreicht abermals die Vulnerabilität, die das sensible Thema mit sich bringt und bei den Lehrkräften hervorgerufen wird: „Ich glaube für die Institution Schule, wenn ich jetzt die Sozialarbeiterin rausnehme, ist das ein Problem, wo ich nicht unbedingt sehe, dass das ,ne Schule lösen kann“ (FS5_L1_110). Im Gegensatz dazu steht die Verantwortung, die die Lehrkräfte ihren Schüler*innen gegenüber empfinden und die den Druck auf die Einzelpersonen zusätzlich erhöht: „Also es gibt schon ganz viele Sachen, die irgendwo untergehen, wo sich keiner drum kümmert. Was uns schon so beschäftigt“" (FS2_6).

Ergänzend zu den eingeschränkten Ressourcen und Kompetenzen der Lehrkräfte, 
insbesondere aber den Mangeln des System Schule im Kontext sexualisierter Gewalt, äußern die Befragten, dass es sie zusätzlich verunsichert, dass sie die Situation der Schulsozialarbeitenden an ihren Schulen als prekär erleben: „Ich denke, das sind immer wieder diese finanziellen Zwänge, müssen jedes Mal zittern um die Stellen, ob das bleibt oder nicht, je nachdem, welcher Politiker gerade mit welcher Idee vorne ran schwebt“ (FS5_127). Eine der wenigen als kompetent erlebten Personengruppen innerhalb des eigenen Kollegiums wird damit zu einer möglicherweise nicht dauerhaft verfügbaren Anlaufstelle, was die Situation zusätzlich verschärft.

\section{Zusammenfassung der Auswertung von Kategorie F2.4, F2.7, F2.8}

Lehrkräfte fühlen sich hinsichtlich des Umgangs mit Verdachtsfällen sexualisierter Gewalt nicht kompetent. Aufgrund fehlender Inhalte im Studium mangelt es an Faktenwissen, vor allem aber auch an Handlungskompetenzen. Nichtsdestotrotz sind sie im Verdachts- oder Disclosure-Fall in der Verantwortung zu handeln und tun dies auch. Da es keine institutionell vorgegebenen Verfahrensabläufe gibt, müssen Lehrer*innen in diesen Fällen selbst entscheiden, welche Maßnahmen sie unternehmen. Dies bringt sie in eine Situation, in der sie sich mitunter überfordert fühlen und nicht sicher sind, ob ihr Handeln richtig ist. Die daraus resultierende Angst, etwas falsch zu machen und dafür gar rechtlich belangt werden zu können, führt dazu, dass Lehrkräfte in ihrer Position als vertrauensvolle Ansprechpersonen hochgradig vulnerabel sind. In Anspruch genommene Unterstützungsmöglichkeiten von externen Stellen werden als nicht ausreichend wahrgenommen und verschärfen das Gefühl der Vulnerabilität eher zusätzlich. Die mangelnden, nicht vorhandenen oder nicht sicheren internen Angebote (Supervision, Schulsozialarbeit, Handlungsempfehlungen) können die hohe psychische Belastung, die mit der Verantwortung zum Umgang mit Verdachtsfällen einhergeht, nur unzureichend auffangen. Lehrer*innen haben deshalb das Gefühl, derartige Situationen nicht adäquat bewerkstelligen zu können und sehen deshalb die Institution Schule nicht in der Lage, sich dieser Problematik annehmen zu können.

\subsubsection{Auswertung der Kategorien F2.2, F2.3 und F2.6}

Dieses Unterkapitel beleuchtet noch einmal gesondert die Kompetenzen von Lehrkräften hinsichtlich Themen Sexueller Bildung, die ihnen im Schulalltag begegnen. In Abgrenzung zur Kategorie F1.8, welche Auskunft über Aussagen zu bekannten Kooperationspartner*innen gibt, fasst die Kategorie F2.6 dabei die Kompetenzen von Lehrkräften zusammen, Schüler*innen, deren Eltern oder andere Personen an diese oder andere Kooperationspartner*innen zu verweisen. Hervorzuheben ist die sehr offene Haltung von Lehrkräften gegenüber Stellen, die bei Unklarheiten zu Rate gezogen werden können. So 
antwortet eine befragte Person auf die Frage, wie sie die eigenen Kompetenzen einschätzen würde: „Eher schlecht. Ich würde mich weiterwenden“ (F3_50). Augenscheinlich resultiert die Offenheit für außenstehende Ansprechpersonen aus der Annahme, dass es unter ihnen Fachkräfte gibt, die im Umgang mit beispielweise Verdachtsfällen kompetenter sind als die Interviewten selbst. Hinzu kommt die Erkenntnis, dass auch die während der Berufstätigkeit erworbenen Kompetenzen (Kategorie F2.2) insbesondere aus Inputs resultieren, die die Befragten außerhalb der Institution Schule erhalten haben. Es liegt demzufolge nahe, dass Wissen über Inhalte Sexueller Bildung nur in speziellen Settings und nicht im alltäglichen schulischen Ablauf erworben werden kann. Die Institution Schule schafft offenbar keine Räume, in denen Lehrkräfte sich erfolgreich bezüglich Themen Sexueller Bildung weiterbilden können: „Ich war zur Gesprächsschulung nochmal, das waren 3 Tage, (...) da hatten wir nochmal hier diese ganzen Bausteine und die mussten wir auch nochmal ausprobieren, da ist das nicht in 120min erledigt" (F5_L1_182). Wenngleich es dann auch mitunter schwierig ist, die für die jeweilige Situation geeignete Stelle zu finden, an die verwiesen werden kann, ist es dennoch nötig und wird auch von den Lehrkräften angestrebt: „Und da sind wir schon gut, wenn wir wirklich den Ansprechpartner finden, der auf den ganz speziellen [Fall] zutrifft, ja, es ist also manchmal schon ein Suchen, wer könnte jetzt der beste Partner sein für solche Geschichten“ (F5_L1_132).

Auf die Frage danach, welche persönlichen Kompetenzen sie mitbringen würden, um überhaupt im Kontext Sexueller Bildung tätig zu sein (Kategorie F2.3), fallen Beschreibungen wie feinfühlig oder sensibel sein, zuhören können, ein Gespür haben oder ein persönliches Anliegen damit verbinden, sich in diesem Bereich zu engagieren. Dennoch ist festzuhalten, dass dieser Kategorie lediglich neun Aussagen zugeordnet werden konnten ${ }^{14}$ und die Lehrkräfte selbst die Frage nach ihren Ressourcen nutzen, um deutlich zu machen: „Also ich würde immer in einem Gespräch versuchen rauszufinden. Anderes eigentlich, ich hab keine anderen Kompetenzen“ (F2_100) (vgl. Kapitel 6.1.6).

\subsubsection{Auswertung der Kategorien F4.1, F4.2, F4.3 und F4.4}

Ein wesentlicher Bestandteil der geführten Interviews fokussiert auf Bedarfe von Lehrkräften an Fort- und Weiterbildung. Alle dahingehend zugeordneten Interviewauszüge sollen an dieser Stelle gemeinsam ausgewertet werden, um einen umfassenden Überblick zu vermitteln, welche Bedarfe Lehrkräfte äußern, aber auch, welche Aktivitäten sie vielleicht bereits selbst unternommen haben, um sich fortzubilden. Dabei ist zunächst festzustellen, dass es kein schulübergreifendes Vorgehen hinsichtlich Fort- und Weiterbildung im Kontext Sexuelle

14 Zum Vergleich: Bei Kategorie F3.7 waren es beispielsweise 60 Textstellen, die entsprechende inhaltliche Bezüge aufwiesen. 
Bildung und sexualisierte Gewalt gibt ${ }^{15}$. Die Bandbreite der Aussagen zu besuchten Weiterbildungen reicht davon, dass Lehrer*innen keinerlei Erfahrungen mit derartigen Angeboten gemacht haben bis hin dazu, dass sogar schulinterne Fortbildungen zur Thematik stattfanden: „Wir hatten vor einem Jahr, vielleicht doch ein bisschen länger her, jemanden hier, die hat mit uns so eine Weiterbildung gemacht zu diesem, wie verfahre ich, wenn ich sowas erfahren habe“ (FS6_68).

Gleichzeitig ist den Codings jedoch auch zu entnehmen, dass die wenigsten Lehrer*innen aktiv nach derartigen Weiterbildungen suchen. Wenn die Angebote nicht in den Schulen selbst stattgefunden haben oder in festgelegten besonderen Settings Bestandteil waren (zum Beispiel bei der Ausbildung zumzur Beratungslehrer*in), gab es lediglich zwei Personen, die auf eigene Initiative hin entsprechende Veranstaltungen besucht haben ${ }^{16}$. Auffällig ist weiterhin, dass alle in Anspruch genommenen Angebote - unabhängig davon, ob schulintern oder -extern - klar auf Kompetenzen zum Handeln in Verdachtsfällen von sexualisierter Gewalt und damit Intervention zielten: „Also es geht da natürlich meistens, das muss ich auch nochmal ganz konkret sagen (...), das geht da schon mehr um dieses Erkennen von wirklichem Missbrauch, sexuellen Missbrauchsfällen. Und was wir da sozusagen tun können, was da unsere Möglichkeiten, Rechte, Pflichten sind“ (F4_128). Es gibt keine zusätzlichen Berichte über Teilnahmen an Fort- oder Weiterbildungen zu sonstigen Inhalten Sexueller Bildung. Keine der befragten Personen hat sich zu Sexueller Bildung ohne den Fokus Intervention im Verdachtsfall weitergebildet.

Was steht dem Besuch von entsprechenden Fort- und Weiterbildungsinhalten entgegen? Die Frage danach beantworten die Interviewten nicht eindimensional. Zunächst könnte sich die Überlegung auftun, ob die Lehrer*innen schlicht keinen Bedarf sehen, um sich zu Inhalten Sexueller Bildung ${ }^{17}$ fortzubilden. Nachdem die Lehrkräfte jedoch bereits sehr reflektiert ihre fehlenden Kompetenzen in diesem Feld erläutert haben (vgl. Kapitel 6.1.6), zeigen sie auch an möglichen Weiterbildungen großes Interesse: „Das ist ja wirklich ein dermaßen sensibler Bereich (...). Ich denke, da kann ich auch im Namen der allermeisten Kollegen sprechen, da wünschen wir uns schon Know-How“ (F4_132). Konkret benannte gewünschte Inhalte konzentrieren sich in den Interviews schließlich zum einen auf Faktenwissen zu Rechten und Pflichten. Dabei finden Fragen dazu Erwähnung, was gesetzlich erlaubt ist, wenn man von sexualisierter Gewalt Kenntnis erlangt, aber auch, woran genau Betroffene für Lehrkräfte

15 ebenfalls scheint es kein einheitliches schulinternes Vorgehen zu geben, mit Ausnahme der Fortbildungsangebote, die für das gesamte Kollegium in der Schule offeriert wurden

${ }^{16}$ Allerdings muss an dieser Stelle erwähnt werden, dass eine der beiden Personen „lediglich“ von einer Veranstaltung zu sexuell übertragbaren Krankheiten berichtet

17 einschließlich Präventions- und Interventionsstrategien sowie Handlungskompetenzen im Verdachtsfall 
möglicherweise erkennbar sind und die Notwendigkeit, noch einmal intensiver zu beleuchten, was genau sexualisierte Gewalt ist: „Wenn ich von jemandem was erfahre, wo wende ich mich denn hin? Wo mache ich mich denn strafbar, wenn ich das Kind dann näheres gefragt habe oder so? So'ne Sachen, die kennen wir ja überhaupt nicht“ (F2_27). Am deutlichsten wird jedoch der Bedarf an Handlungskompetenzen im Umgang mit von sexualisierter Gewalt betroffener Kinder und Jugendlicher signalisiert. Dabei werden vor allem Aspekte der Gesprächsführung benannt, aber auch der Wunsch nach „Handwerkszeug“ für das Einbeziehen von Bezugspersonen thematisiert ebenso wie das Anliegen, die eigenen Verweisungskompetenzen zu verbessern und zuständige Stellen zu kennen: „Wie geht man mit diesem Wissen, wenn sie sich anvertrauen, um? Was kann man machen? Wo kann man sie hinschicken? In welche Richtung könnte das gehen“ (F1_51). Der große Schulungsbedarf zu Interventionsstrategien, -maßnahmen und -kompetenzen deckt sich mit dem Auswertungsergebnis, dass es im System Schule einen Mangel an institutionell vorgegebenen Vorgehensweisen gibt.

Trotz formulierter Bedarfe besuch(t)en die Befragten jedoch keine Fort- und Weiterbildungsangebote. Die Analyse der Codings von Kategorie F4.1 - F4.4 vermittelt nur zum Teil den Eindruck, dass die Lehrkräfte keinen Zugang zu Angeboten Sexueller Bildung haben. Personen, die den Zugang als problematisch benennen, wünschen sich beispielsweise, deutlicher auf etwaige Fortbildungen zum Thema hingewiesen zu werden: „Also meinetwegen, dass eine E-Mail an Schulen kommt. Oder, ja, dass das nicht zwischen dreihundert anderen Weiterbildungen steht“ (F2_33). Der in diesem Zitat ebenfalls anklingende Aspekt der Fülle an möglichen Weiterbildungsinhalten und damit auch an für den Schulalltag relevanten Themen bietet Anlass hinterfragt zu werden, wird aber von den Interviewten in dieser Form nicht verfolgt. Worauf jedoch mehrfach verwiesen wird, ist die Tatsache, dass die organisatorischen Bedingungen für den Besuch derartiger Weiterbildungen für die Lehrer*innen nicht mit ihrer alltäglichen Arbeit zu vereinbaren sind. Dabei spielen die Erreichbarkeit der Veranstaltungsorte eine Rolle, vor allem aber zeitliche Aspekte. Lehrkräfte zeigen sich zwiegespalten zwischen dem Bedarf, den sie erkennen und der Relevanz, die sie der Thematik zuschreiben und ihren zeitlichen und persönlichen Ressourcen, die sie unabhängig von Bedarf und Relevanz - aufzubringen im Stande sind: „Ich hatte einen langen Tag gehabt, ich hatte Unterricht, dann hatte ich jetzt meine Dienstberatung, jetzt bin ich hier. Und wenn man dann einen langen Tag Unterricht hatte und kommt dann zu einer Fortbildung, dann ist das doch anstrengend und man ist nicht mehr so aufnahmefähig und vielleicht nicht mehr aufnahmebereit. Wochenende stört es, weil man möchte ja sein freies Wochenende haben oder auch unterrichtsfreie Zeit“ (F3_61). Eine Person fasst die Problematik wie folgt zusammen: „In unserem Schulrahmen gibt es immer zwei Varianten: man kann das nach dem Unterricht machen, da kann man maximal noch zwei Stunden etwas erzählen, weil dann sind 
die Lehrer, wenn die sechs Stunden Unterricht gemacht haben, auch einfach fertig. Und man kann das halt in einem größeren Rahmen machen, also sprich in einer Tagesveranstaltung, ist aber unrealistisch, weil das muss man dann in der Regel irgendwo in die Ferien legen, das ist wiederum auch/ also weil man braucht ja einfach die, naja, also die Bereitschaft, auch sozusagen die Motivation der Kollegen dafür“ (F4_142). Die zeitlichen Kapazitäten der Lehrer*innen stehen dabei in deutlichem Gegensatz dazu, dass sie es als besonders hilfreich ansehen, in Angeboten zu Fort- und Weiterbildung neben dem theoretischen Input auch praktische Bezüge herzustellen, „weil das ist was, was man sich dann merkt. Während viel Anderes, was so theoretisch ist, das ist dann zwar im Moment nicht schlecht und vielleicht hat man es auch noch ,ne Weile, aber es ist nicht so offensichtlich parat wie anderes Wissen" (F5_L1_184). Mehrfach jedoch wird (wie bereits bei oben genanntem Zitat F4_142) auch die Bereitschaft der Pädagog*innen in Frage gestell ${ }^{18}$, sich auf entsprechend zeitintensive Weiterbildungssettings mit diesen Inhalten einzulassen.

\section{Zusammenfassung der Auswertung von Kategorie F4.1, F4.2, F4.3 und F4.4}

Es gibt kein institutionell festgeschriebenes Fort- und Weiterbildungsbestreben von Schulen für ihre Lehrkräften. Die Mehrheit der Lehrer*innen hat bisher keine Fort- und Weiterbildungen zu den Themen Sexuelle Bildung und sexualisierte Gewalt aus eigener Initiative heraus besucht. Gelegentlich gab es Schulungsangebote in den Schulen selbst, die dem gesamten Kollegium offenstanden. Keine der Fortbildungen, die in den Interviews zu Sprache kam, fokussierte konkret auf Inhalte Sexueller Bildung. Stattdessen setzten alle genannten und in Anspruch genommenen Weiterbildungsangebote dabei an, im Verdachtsfall sexualisierter Gewalt kompetent intervenieren zu können. Dies deckt sich mit den Bedarfen, die die Lehrkräfte im Hinblick auf mögliche Inhalte von derartigen Veranstaltungen formulieren. Auch diese stellen insbesondere Handlungskompetenzen wie Gesprächsführung mit Betroffenen oder aber rechtliche Aspekte im Verdachtsfalls heraus.

Was genau dem Besuch von Weiterbildungen zur Thematik entgegensteht, kann anhand des Datenmaterials nicht konkret eruiert werden. Deutlich wird jedoch, dass die Lehrkräfte zum einerseits einen klaren Bedarf an Fort- und Weiterbildung formulieren, die zur Verfügung stehenden Angebote hinsichtlich ihrer strukturellen Bedingungen sich häufig aber nicht mit den zeitlichen und persönlichen Ressourcen der Befragten decken.

\footnotetext{
18 damit ist auch die eigene Bereitschaft der befragten Personen gemeint
} 


\subsubsection{Die übrigen Kategorien}

Wie dem Kategoriensystem zu entnehmen, wurden nicht alle Kategorien im Rahmen der vorliegenden Auswertung betrachtet. Den in dieser Masterarbeit nicht ausgewerteten Kategorien konnten im Rahmen der Datenmaterialanalyse entweder zu wenig aussagekräftige Textstellen zugeordnet werden (F3.3, F3.4, F3.5) oder eine Auswertung wäre aufgrund sonstiger Faktoren nicht inhaltlich aufschlussreich gewesen. Auf zwei der entsprechenden Kategorien soll dennoch stellvertretend - die nicht erfolgte „richtige“ Auswertung erläuternd eingegangen werden.

Gemäß Kategoriensystem sollte die Kategorie F3.8 das Verständnis Sexueller Bildung der befragten Personen eruieren. Neben der Tatsache, dass es nur marginal überhaupt konkrete Aussagen zu Sexueller Bildung in den Interviews gibt, ergibt sich aufgrund der vorgenommenen Auswertung eine weitere Annahme: Die Summe anderer Kategorien gibt einen detaillierten Überblick darüber, welches Verständnis Sexueller Bildung dem Handeln von Lehrkräften zugrunde liegt. Zwar fokussiert keine der sonstigen Kategorien auf Sexuelle Bildung, jedoch skizziert die Gesamtbetrachtung der Auswertung ein Bild davon, was Lehrkräfte mit Inhalten Sexueller Bildung verbinden. Da keine der befragten Personen wirklich konkrete Aussagen zum Konzept der Sexuellen Bildung macht, ist eine separate Auswertung der Kategorie F3.8 nicht zielführend und wird daher nicht vorgenommen.

Ähnlich verhält es sich mit Textpassagen, die die von Lehrer*innen wahrgenommene Grenzen in ihrer täglichen Arbeit zum Themengebiet Sexualität und sexuelle Selbstbestimmung widerspiegeln (Kategorie 3.6) Es ist festzuhalten, dass die meisten Grenzen, die Lehrer*innen in Bezug auf diese Thematik formulieren, in der Auswertung bereits an anderer Stelle benannt wurden. Wenige zusätzliche Codings ergänzen das entstandene Bild dahingehend, dass auch die individuelle Arbeitsbelastung der Lehrkräfte beziehungsweise der Druck, vorgesehenen Unterrichtsstoff vermitteln zu müssen, als Grenze dahingehend erlebt wird, mit den Schüler*innen zum Thema Sexualität zu arbeiten. Inwieweit dies jedoch entscheidend dafür ist, wie Lehrkräfte Inhalte Sexueller Bildung an Kinder und Jugendliche vermitteln und inwiefern diese Grenze Einfluss darauf nimmt, die Schule zum Schutzraum vor sexualisierter Gewalt zu gestalten, kann an dieser Stelle nicht beantwortet werden. Aus diesem Grund werden auch keine zusätzlichen Ableitungen dahingehend getroffen.

\subsection{Kernaussagen und zentrale Thesen der Auswertung}

Der vorhergegangenen Auswertung sind zentrale Kernaussagen und Thesen zu entnehmen, die im Folgenden gesammelt abgebildet werden sollen. Im späteren Verlauf sollen sie als Grundlage zur Beantwortung der Forschungsfragen herangezogen werden. An dieser Stellen bietet es sich jedoch an, sie zunächst unkommentiert und unterteilt in zwei Gruppen zu präsentieren. In der ersten Gruppe werden Aussagen der interviewten 
Lehrer*innen rekonstruktiv wiedergegeben, bilden also konkret das in den Interviews Gesagte ab. Die zweite Gruppe vereint Thesen, die im Anschluss an die Auswertung von einer Außensicht formuliert wurden.

\subsubsection{Rekonstruktive Kernaussagen}

Lehrer*innen erkennen an, dass die Institution Schule sich an der Sexualaufklärung von Kindern und Jugendlichen beteiligen muss.

Lehrer*innen äußern ein sehr sensibles Verständnis von sexuellen Grenzverletzungen. Dies deckt sich nicht immer mit der Wahrnehmung der Schüler*innen, die insbesondere verbale Übergriffe häufig nicht als Grenzverletzung wahrnehmen.

Schüler*innen benötigen ein vertrauensvolles Umfeld, um sich anzuvertrauen. Lehrkräfte möchten ihnen dies zur Verfügung stellen.

Lehrer*innen empfinden es als notwendig und wichtig, dass schulische Präventionsangebote im Kontext sexualisierter Gewalt darauf fokussieren, dass Kinder und Jugendliche lernen, ihre Bedürfnisse und Grenzen wahrzunehmen und sich für diese einzusetzen.

Lehrkräfte kooperieren mit schulexternen und -internen Ansprechpartner*innen, um Themen Sexueller Bildung zu bearbeiten oder in Problemsituationen handlungsfähig zu sein.

Lehrer*innen erleben die Kooperationen mit externen Personen häufig als einseitige, d.h. nur von ihnen in Anspruch genommene Angebote. Die Lehrer*innen werden nicht in den weiteren Verlauf des Angebots involviert. Zum Teil ist dies in ihrem Interesse, zum Teil erleben sie es als Nichtachtung ihrer Kompetenzen.

Die Situation von Schulsozialarbeitenden ist prekär, ihre Stellen sind nicht sicher. Dies verunsichert Lehrkräfte zusätzlich.

Lehrer*innen empfinden sich hinsichtlich Faktenwissens und Handlungskompetenzen im Umgang mit Verdachtsfällen sexualisierter Gewalt als nicht kompetent.

Lehrkräfte erleben andere Lehrkräfte nicht als kompetent im Hinblick auf Themen Sexueller Bildung oder Verdachtsfälle von sexualisierter Gewalt.

Lehrkräfte nehmen die Sexualität von Schüler*innen als Gefahr für deren Zukunft(sgestaltung) wahr. Dieser Gefahr soll schulische Sexualerziehung entgegenwirken.

Lehrer*innen erleben die Zusammenarbeit mit Eltern häufig als problematisch. 


\subsubsection{Zentrale Thesen auf Grundlage der Auswertung}

Wie aktiv und intensiv Themen Sexueller Bildung im unterrichtlichen Rahmen behandelt werden, ist nicht strukturell verankert und damit individualisiert, also abhängig von der zuständigen Lehrkraft. Auch die Kooperation mit externen oder internen Ansprechpersonen ist nicht institutionell festgelegt und deshalb ebenfalls individualisiert.

Es fällt Lehrkräften leichter, bezüglich Themen von Sexualität zu intervenieren als selbst präventiv tätig zu werden.

In Problemsituationen sind Klassenlehrer*innen zentrale Ansprechpersonen von Schüler*innen.

Die Schulleitung ist in unklaren Situationen im Kontext sexualisierter Gewalt eine wichtige Ansprechperson. Schulleiter*innen werden als kompetent erlebt und kennen weitere Verfahrensabläufe.

Offensichtliche körperliche sexuelle Grenzverletzungen in schulischen Institutionen haben keinen Verhandlungsspielraum und ziehen eine sofortige Intervention nach sich.

Lehrer*innen können sich im Verdachtsfall nicht an institutionellen Handlungsleitfäden orientieren. Es gibt kein einheitliches Vorgehen. Die Vorgehensweise ist abhängig von den beteiligten Personen.

Die Entscheidung einer Lehrkraft darüber, welche Intervention auf eine sexuell übergriffige Handlung von Schüler*innen folgt, ist nicht nur abhängig von der Grenzverletzung selbst. Auch das Setting (wer ist unter welchen Bedingungen übergriffig geworden) ist entscheidend.

Schüler*innen können die Involvierung Dritter durch die Lehrkraft als Vertrauensbruch erleben. Fehlende institutionelle Handlungsanweisungen können Lehrkräfte in Gewissenskonflikte bringen.

Fehlende strukturell verankerte Handlungsanweisungen bezüglich des Vorgehens im Verdachtsfall machen Lehrer*innen vulnerabel. Sie haben Sorge, falsch zu handeln. Diese Sorge reicht bis zur Angst vor strafrechtlicher Verfolgung aufgrund fehlerhaften Umgangs mit Betroffenen sexualisierter Gewalt.

Schulsozialarbeitende haben eine zentrale Funktion hinsichtlich Angeboten Sexueller Bildung, aber auch bei der Bearbeitung von Problemstellungen (beispielsweise Verdachtsfällen).

Die Inhalte Sexueller Bildung in der Lehramtsausbildung sind ungenügend.

Fehlende Möglichkeiten der Unterstützung durch zum Beispiel Schulsozialarbeiter oder Supervision verstärkt die Vulnerabilität der Lehrkräfte. 
Angebote schulischer Sexualaufklärung fokussieren auf biologisch-medizinische und soziale Aspekte. Zentrale Themen sind Schutz vor sexuell übertragbaren Krankheiten und insbesondere der Schutz vor frühzeitigen Schwanger- beziehungsweise Elternschaften.

Fort- und Weiterbildungsveranstaltungen zum Themenkomplex Sexuelle Bildung erreichen Lehrkräfte bisher nur punktuell. In Anspruch genommene Angebote fokussieren auf Interventionskompetenzen im Verdachtsfall von sexualisierter Gewalt.

Vorhandene Fort- und Weiterbildungsangebote sind nicht passgenau hinsichtlich der persönlichen und zeitlichen Ressourcen, die Lehrer*innen dafür aufbringen können.

Der von den Lehrkräften formulierte Fort- und Weiterbildungsbedarf und die Relevanz sind konträr zu zeitlichen, organisatorischen und persönlichen Ressourcen und zum Teil Motivationen der Pädagog*innen.

\subsection{Beantwortung der Forschungsfragen}

Nach der erfolgten Auswertung sollen an dieser Stelle die zuvor formulierten Forschungsfragen beantwortet werden. Da sich die Antwortinhalte der einzelnen Forschungsfragen zum Teil deutlich überschneiden, erfolgt die Beantwortung in einem zusammenfassenden Fließtext.

Lehrer*innen sind sich ihrer Verantwortung bewusst, im Rahmen ihrer Tätigkeit im Schulbetrieb einen Beitrag zur Sexualaufklärung von Kindern und Jugendlichen leisten zu müssen. Um diesem Auftrag gerecht zu werden, sollten Angebote Sexueller Bildung im Schulalltag dazu beitragen, dass Schüler*innen hinsichtlich ihrer sexuellen Selbstbestimmung gestärkt werden und sich erfolgreich gegen sexualisierte Gewalt wehren können. Aktuell hat sich das Konzept der Sexuellen Bildung mit einem Fokus auf sexuelle Selbstbestimmung und Prävention sexualisierter Gewalt noch nicht in schulischen Institutionen etabliert. Es gibt keinen strukturellen Rahmen, der festlegt, wie und in welchem Umfang Angebote Sexueller Bildung und der Prävention sexualisierter Gewalt in den Unterricht integriert werden müssen. So kommt es, dass Lehrer*innen nur punktuell Angebote zur schulischen Sexualerziehung unterbreiten oder Faktenwissen zur Sexualaufklärung vermitteln. Da entsprechende Inhalte nicht festgelegt, also standardisiert sind, sind Form, Fokus, Umfang etc. maßgeblich abhängig von der Initiative der zuständigen Lehrkraft. Aktuell liegt das Hauptaugenmerk der Sexualaufklärung vorrangig darauf, biologisch-medizinische Kenntnisse zu Körper, Sexualität, Krankheiten und Fortpflanzung zu vermitteln. Ein wichtiger Aspekt schulischer 
Sexualaufklärung ist es, über die Gefahr frühzeitiger Schwangerschaften zu informieren und frühe Elternschaften zu verhindern.

Sexuell aktive Schüler*innen sind in den Augen von Lehrer*innen gefährdet, durch frühe Schwanger- beziehungsweise Elternschaften die Möglichkeiten zur Gestaltung ihrer Zukunft einzuschränken. Schulische Sexualerziehung und Sexualaufklärung soll diese Gefahr mindern und zielen nicht vorrangig auf Bestärkung zur sexuellen Selbstbestimmung.

Angebote zur Prävention sexualisierter Gewalt werden in den meisten Schulen bisher nur punktuell in den Schulalltag integriert. Finden sie statt, sollen sie insbesondere dazu beitragen, dass die Kinder und Jugendlichen lernen, ihre eigenen Grenzen wahrzunehmen und sie (auch anderen gegenüber) zu verbalisieren. Zwar werden zur Realisierung derartiger Projekte externe Kooperationspartner*innen wie beispielsweise Fachberatungsstellen angefragt. Jedoch gibt es auch hinsichtlich der Einbeziehung von außerschulischen Trägern keine Einheitlichkeit, sodass die tatsächlich angebotenen Präventionsmaßnahmen sich von Schule zu Schule, mitunter sogar von Klasse zu Klasse, ebenfalls deutlich voneinander unterscheiden. In den seltensten Fällen können Lehrer*innen selbst Schuleinheiten zu Prävention oder sexueller Selbstbestimmung anbieten, was darin begründet ist, dass sie in ihrer Ausbildung nur unzureichend darauf vorbereitet werden. Das Lehramtsstudium beinhaltet kaum bis gar keine Inhalte Sexueller Bildung und befähigt angehende Lehrkräfte nicht, sich im späteren Schulbetrieb entsprechend zu engagieren.

Lehrer*innen empfinden sich deshalb als nicht gut ausgebildet und nicht kompetent darin, den Schüler*innen Angebote Sexueller Bildung zu unterbreiten.

Die Inhalte, die sie dennoch in ihren Unterricht einbauen, resultieren oftmals aus einem persönlichen Interesse für das Thema oder der Initiative einzelner Personen in den Schulen.

Aufgrund fehlender struktureller Verankerungen von Präventionsangeboten und Handlungsabläufen in Verdachtsfällen, wirkt die Institution Schule bisher nur marginal präventiv und trägt nicht effektiv zum Schutz von Kindern und Jugendlichen vor sexualisierter Gewalt bei.

Dies bedeutet allerdings nicht, dass sich die Lehrkräfte dafür nicht verantwortlich fühlen oder in in Verdachtsfällen nicht tätig werden. Im Gegenteil: Lehrer*innen empfinden eine große Verantwortung dafür, zum Wohlergehen ihrer Schüler*innen beizutragen - insbesondere in der Rolle als Klassenlehrer*innen, die sie zu zentralen Ansprechpersonen der Kinder und Jugendlichen macht. Mit großem Engagement versuchen sie vertrauensvolle Räume zu schaffen, die es ermöglichen, dass Schüler*innen sich mit den Themen an sie wenden, die sie bewegen. Das Verantwortungsgefühl führt unter anderem dazu, dass Lehrer*innen sehr sensibel für sexualisierte Grenzverletzungen an Schüler*innen sind. Insbesondere bei 
offensichtlichen körperlichen, also auch sexualisierten, Übergriffen gibt es für die Pädagog*innen keinen Verhandlungsspielraum hinsichtlich sofort folgender Konsequenzen für die übergriffige Person.

Auch Interventionen in sonstigen (Verdachts-)Fällen sexualisierter Gewalt stehen für die Lehrer*innen außer Frage. Auf akute Vorkommnisse zu reagieren, also zu intervenieren, fällt innen deutlich leichter, als präventiv tätig zu werden. Dennoch mangelt es auch hinsichtlich des Vorgehens im Verdachtsfall oder im Falle einer Aufdeckung von sexualisierter Gewalt an institutionellen Handlungsanweisungen. Zwar führen fehlende Handlungskompetenzen, die Lehrer*innen bei sich selbst einschätzen, nicht zu einer Handlungsunfähigkeit. Die fehlenden einheitlichen Regelungen individualisieren jedoch auch die Interventionen und machen sie maßgeblich abhängig von den Handlungskompetenzen der involvierten Lehrkraft.

Damit ist nicht sichergestellt, dass jedem Kind oder jedem*jeder Jugendlichen die Unterstützung zukommt, die im Falle des Bekanntwerdens von sexualisierter Gewalt benötigt wird.

Dies betrifft sowohl Schüler*innen, die sexualisierte Gewalt erleben als auch Heranwachsende, die selbst übergriffig werden. Je nach situativer Einschätzung der beteiligten Lehrkraft kann das daraus resultierende Handeln sich deutlich unterscheiden, die Intervention hilfreich und angemessen sein oder nicht sein. Dies setzt nicht nur die Schüler*innen, sondern auch die Lehrer*innen zusätzlichen Gefahren aus und macht sie vulnerabel. Mögliche Gefahren reichen von empfunden Vertrauensbrüchen auf Seiten der Schüler*innen und daraus resultierenden Gewissenskonflikten ${ }^{19}$ auf Seiten der Lehrpersonen bis hin zur Sorge vor rechtlichen Verfehlungen aufgrund von Unwissenheit ${ }^{20}$. Viele im Schulbetrieb tätige Lehrpersonen suchen in solchen (Verdachts-)Fällen von sexualisierter Gewalt interne Unterstützung bei ihrer Schulleitung oder - wenn vorhanden - der*dem Schulsozialarbeiter*in. Beide Personengruppen werden von den Lehrkräften - im Gegensatz zu anderen Kolleg*innen im Kollegium - als sehr kompetent erlebt, hinsichtlich des weiteren Vorgehens behilflich sein zu können. Jedoch sind zumindest die Schulsozialarbeitenden keine sichere Größe, auf die die Ratsuchenden dauerhaft bauen können: Mitunter sind an Schulen gar keine Schulsozialarbeitenden beschäftigt oder ihre Stellen sind prekär, stehen also immer wieder auf dem Prüfstand. Dies verunsichert Lehrer*innen zusätzlich, was ihre Vulnerabilität noch erhöht. Auf zusätzliche Angebote wie Supervision oder konzeptionell festgelegte kollegiale Austausche zu derartigen Themen können Lehrkräfte nicht zurückgreifen, es gibt

19 Dies ist beispielsweise der Fall, wenn entgegen der Erwartung und vorhandener oder nicht vorhandener Absprachen dritte Personen einbezogen werden.

20 Lehrer*innen haben Angst, sie könnten beispielsweise im Falle einer Aufdeckung rechtliche Verfehlungen durch zu intensive Gespräche mit den betroffenen Kindern und Jugendlichen begehen. 
sie schlicht nicht.

Akute Abhilfe für diese Situation könnten in erster Linie Fort- und Weiterbildungen von Lehrkräften zum Themenfeld Sexualität schaffen. Bisher erreichen derartige Angebote die Lehrer*innen kaum und auch hier insbesondere auf Initiative einzelner Personen - entweder der Lehrkräfte selbst oder sonstiger engagierter Personen, die beispielsweise Veranstaltungen für das Gesamtkollegium arrangieren.

Die wenigen in Anspruch genommenen Angebote fokussieren auf Interventionen der Lehrkräfte in Verdachtsfällen von sexualisierter Gewalt. Fort- und Weiterbildungsangebote befähigen die Lehrkräfte aktuell nicht zur Umsetzung präventiver Maßnahmen.

Generell bedarf es spezieller Settings, um Lehrer*innen Inhalte Sexueller Bildung zu vermitteln. Häufig mangelt es bestehenden Angeboten daran, dass sie nicht den persönlichen und zeitlichen Ressourcen von Lehrkräften entsprechen. Obgleich die Mehrheit von ihnen durchaus an der Thematik interessiert und bereit ist, sich fortzubilden, können weiterbildende Veranstaltung deshalb oft nicht besucht werden. 


\section{Fazit und Ausblick}

Sowohl die Wissenschaft als auch die schulische Praxis sind sich einig darin, dass die Institution Schule hinsichtlich der sexuellen Sozialisation von Kindern und Jugendlichen ein zentraler Lern- und Erfahrungsort ist und deshalb einen Beitrag zur Sexualaufklärung und Sexualerziehung von Schüler*innen leisten muss. Neben der Vermittlung von Kompetenzen zur sexuellen Selbstbestimmung ist die Institution Schule ebenfalls gefordert, die Prävention von sexualisierter Gewalt zu fördern und damit zum Schutz von Kindern und Jugendlichen vor sexuellen Grenzverletzungen beizutragen. Die zentralen Personen, die sich im Schulalltag dieser Herausforderung stellen müssen, sind Lehrer*innen aller Schulformen. Die vorliegende Untersuchung hat gezeigt, dass es den Lehrkräften nicht an Bereitschaft mangelt, dies zu tun. Viel mehr sind sie sehr am Wohlergehen ihrer Schüler*innen interessiert und investieren viel Kraft und Zeit, um sie darin zu unterstützen und in Problemsituationen zu begleiten. Insbesondere für das Handeln in Verdachtsfällen von sexualisierter Gewalt mangelt es Lehrpersonen an Kompetenzen, was vor allem im Fehlen von klaren und institutionell festgelegten Handlungsanweisungen begründet ist. Sowohl das Vorgehen im Verdachtsfall als auch die Unterbreitung von präventiven, aber auch grundlegenden Angeboten Sexueller Bildung sind hochgradig individualisiert und nicht strukturell im System Schule verankert. Dies beschränkt Lehrer*innen nicht nur in ihren Handlungsmöglichkeiten, sondern verunsichert und überfordert sie und macht sie damit vulnerabel.

Obwohl sie dann, wenn ihre Intervention gefragt ist, durchaus handlungsfähig sind, empfinden sich die Lehrkräfte im Umgang mit Fragen zu Sexualität ebenso wenig kompetent wie im gezielten Vorgehen bei Verdachtsfällen von sexualisierter Gewalt. Ein notwendiger Schritt, um sie frühzeitig zu professionellem Handeln zu befähigen und ihnen Basiswissen, aber auch weiterführende Inhalte Sexueller Bildung zu vermitteln, ist die verbindliche Implementierung dieser Themen in das Lehramtsstudium. Die Aufarbeitung der Lücken in der universitären Lehrer*innenausbildung muss zum zeitnahen Ziel gesetzt werden, um den Schutz von Kindern und Jugendlichen vor sexualisierter Gewalt weiter voranzubringen. Erste Maßnahmen, zukünftige, aber auch bereits im Schulbetrieb tätige Lehrer*innen zur Vermittlung von Inhalten sexueller Bildung zu befähigen, werden bereits ergriffen. Eine dieser Maßnahmen ist das vom BMBF geförderte Forschungsprojekt „SeBiLe - Sexuelle Bildung für das Lehramt“. Es wurde im Rahmen der neuen Förderbekanntmachung bewilligt und läuft seit 01.02.2018 als Verbundprojekt in zwei Bundesländern. Bei „SeBiLe“ handelt es sich um ein Forschungsvorhaben im Zusammenhang mit sexualisierter Gewalt gegen Kinder und Jugendliche in pädagogischen Kontexten mit der Absicht, neue Lehrmodule mit dem Fokus auf sexualisierte Gewalt und sexuelle Selbstbestimmung in der Lehramtsausbildung sowie in 
der Fort- und Weiterbildung von Lehrenden und Führungskräften an Schulen zu entwickeln. Dazu sollen bereits bestehende Angebote in Sachsen und Sachsen-Anhalt analysiert werden und im Rahmen einer quantitativen Erhebung die Wissensbestände und Qualifizierungsbedarfe der Studierenden und Absolvent*innen in Lehramtsstudiengängen erfasst werden. Eine punktuelle qualitative Erhebung mit Fachkräften ergänzt die gewonnenen Erkenntnisse. In der zweiten Phase werden Lehrmodule für die Aus-, Fort- und Weiterbildung entwickelt, womit dem tatsächlichen Bedarf von (werdenden) Lehrkräften Rechnung getragen wird.

Die Ausbildung und damit die Befähigung der Lehrer*innen, individualisiert adäquat zu handeln, ist jedoch nur ein Teil der Entwicklung, die notwendig ist, um sowohl Kinder und Jugendliche in ihrer sexuellen Selbstbestimmung zu stärken und vor sexualisierter Gewalt zu schützen als auch Lehrkräften Handlungssicherheit zu geben. Bundesinitiativen wie „Schule gegen sexuelle Gewalt" setzen bei der Institution Schule selbst an, was dringend angezeigt ist. Die Institution Schule muss einen konzeptionellen Rahmen schaffen, durch den Kinder und Jugendliche gestärkt und geschützt werden und in dem Lehrer*innen sich an Handlungsleitlinien orientieren können. Dazu zählen neben informativen und präventiven Angeboten insbesondere auch die Auseinandersetzung mit möglichen Risikofaktoren, die sexualisierte Gewalt in den einzelnen Schulen begünstigt, aber auch mit Ressourcen, die die jeweilige Schule in Form ihres Kollegiums und ihrer Schüler*innenschaft (und deren Eltern) besitzt.

Aktuell schöpft die Institution Schule ihre Möglichkeiten nicht aus, mit der sie sowohl Schutzraum vor sexuellen Grenzverletzungen als auch Ort Sexueller Bildung sein kann. Es mangelt Schulen nicht an Lehrkräften, die bereit sind, sich für diese Belange einzusetzen und sich entsprechend aus-, fort- und weiterzubilden. Es bedarf jedoch einer umfangreichen Fortführung der begonnenen Entwicklungen und einer strukturellen Veränderung der institutionellen Bedingungen in der universitären Lehrer*innenausbildung und den Schulen selbst, um Kinder und Jugendliche in einer positiven Entwicklung zu stärken und sie erfolgreich vor sexualisierter Gewalt und ihren Folgen zu bewahren. 


\section{Literatur}

(1) Arbeitsstab des Unabhängigen Beauftragten für Fragen des sexuellen Kindesmissbrauchs (o.J.): Das Amt. Online verfügbar unter: https://beauftragter-missbrauch.de/derbeauftragte/das-amt/ (Zugriff am 12.01.18, 15:00 Uhr).

(2) Arbeitsstab des Unabhängigen Beauftragten für Fragen des sexuellen Kindesmissbrauchs (o.J.): Schule - Die große Chance für Kinderschutz. Online verfügbar unter: https://www.keinraum-fuer-missbrauch.de/schutzkonzepte/schule/ (Zugriff am 22.06.18, 18:00 Uhr).

(3) Arbeitsstab des Unabhängigen Beauftragen für Fragen des sexuellen Kindesmissbrauchs (o.J.): Definition von sexuellem Missbrauch. Online verfügbar unter: https://beauftragtermissbrauch.de/praevention/was-ist-sexueller-missbrauch/definition-von-sexuellemmissbrauch/ (Zugriff am 21.08.18, 18:45 Uhr).

(4) Arbeitsstab des Unabhängigen Beauftragen für Fragen des sexuellen Kindesmissbrauchs (o.J.): Schutzkonzepte. Online verfügbar unter: https://beauftragtermissbrauch.de/praevention/schutzkonzepte/ (Zugriff am 20.10.18, 17:00 Uhr).

Baader, Meike Sophia (2012): Blinde Flecken in der Debatte über sexualisierte Gewalt. In: Thole, Werner / Baader, Meike / Helsper, Werner / Kappeler, Manfred / Leuzinger-Bohleber, Marianne / Reh, Sabine / Sielert, Uwe / Thompson, Christiane (Hg.): Sexualisierte Gewalt, Macht und Pädagogik. Opladen, Berlin, Toronto: Verlag Barbara Budrich, S. 84 - 101.

Bauer, Ullrich / Kolip, Petra / Finne, Emily / Körner, Wilhelm (2018): Prävention von sexualisierter Gewalt in der Schule. Erste Erfahrungen mit einem niedrigschwelligen Ansatz für Lehrkräfte und Kinder im Grundschulalter. In: In: Wazlawik, Martin / Voß, Heinz-Jürgen / Retkowski, Alexandra / Henningsen, Anja / Dekker, Arne (Hg.): Sexuelle Gewalt in pädagogischen Kontexten. Aktuelle Forschungen und Reflexionen. Springer Fachmedien: Wiesbaden, S. $181-193$.

Bergmann, Christine (2012): Kinder brauchen Aufklärung und Schutzkonzepte. In: Thole, Werner / Baader, Meike / Helsper, Werner / Kappeler, Manfred / Leuzinger-Bohleber, Marianne / Reh, Sabine / Sielert, Uwe / Thompson, Christiane (Hg.): Sexualisierte Gewalt, Macht und Pädagogik. Opladen, Berlin, Toronto: Verlag Barbara Budrich, S. 249 - 263.

Bienstein, Pia / Urbann, Katharina / Scharmanski, Sara / Verlinden, Karla (2018): Prävention sexuellen Missbrauchs an Kindern und Jugendlichen mit Behinderung. Eine Fortbildung für Förderschullehrer_innen. In: Wazlawik, Martin / Voß, Heinz-Jürgen / Retkowski, Alexandra / Henningsen, Anja / Dekker, Arne (Hg.: Sexuelle Gewalt in pädagogischen Kontexten. Aktuelle Forschungen und Reflexionen. Springer Fachmedien: Wiesbaden, S. 211 - 229. 
Bittner, Melanie (2011): Geschlechterkonstruktionen und die Darstellung von Lesben, Schwulen, Bisexuellen, Trans* und Inter* (LSBTI) in Schulbüchern. Eine gleichstellungsorientierte Analyse mit einer Materialsammlung für die Unterrichtspraxis. Frankfurt: Gewerkschaft Erziehung und Wissenschaft.

Blumenthal, Sara-Friederike (2014): Scham in der schulischen Sexualaufklärung. Eine pädagogische Ethnographie des Gymnasialunterrichts. Wiesbaden: Springer Fachmedien.

Bode, Heidrun / Heßling, Angelika (2015): Jugendsexualität 2015. Die Perspektive der 14- bis 25-Jährigen. Ergebnisse einer aktuellen Repräsentativen Wiederholungsbefragung. Bundeszentrale für gesundheitliche Aufklärung, Köln.

Böllert, Karin / Wazlawtik, Martin (Hg.) (2014): Sexualisierte Gewalt: Institutionelle und professionelle Herausforderungen. Wiesbaden: Springer Fachmedien. Online verfügbar unter: https://www.bmbf.de/pub/Sexuelle_Gewalt_gegen_Kinder_und_Jugendliche.pdf (Zugriff am 22.06.18, 18:15 Uhr).

Breitenbach, Eva (2016): Überlegungen zum Workshop „Sexuelle Gewalt als Gegenstand der Hochschullehre“. In: Mahs, Claudia / Rendtorff, Barbara / Rieske, Thomas Viola (Hg.): Erziehung, Gewalt, Sexualität. Zum Verhältnis von Geschlecht und Gewalt in Erziehung und Bildung. Opladen, Berlin, Toronto: Verlag Barbara Budrich, S. $205-210$.

Bründel, Heidrun (2011): Sexuelle Gewalt in schulischen Institutionen. Hintergrund, Analysen, Prävention. Frankfurt: Verlag für Polizeiwissenschaft.

Bundesministerium für Bildung und Forschung (Hg.) (2016): Sexuelle Gewalt gegen Kinder und Jugendliche in pädagogischen Kontexten: Forschung zu Prävention und Schutzkonzepten. Berlin: Bundesministerium für Bildung und Forschung.

Bundesministerium für Bildung und Forschung (Hg.) (2015): Ethische Richtlinien für Forschung zu Kindesmissbrauch. Online verfügbar unter: https://www.bmbf.de/de/ethische-richtlinienfuer-forschung-zu-kindesmissbrauch-1262.html (Zugriff am 13.01.18, 13:00 Uhr).

Bundesministerium für Bildung und Forschung (o. J.): Sexualisierte Gewalt: Kinder und Jugendliche schützen. Online verfügbar unter: https://www.bmbf.de/de/schutz-von-kindernund-jugendlichen-vor-sexueller-gewalt-1241.html (Zugriff am 01.09.2018; 14:30 Uhr).

Bundesministerium für Familie, Senioren, Frauen und Jugend (Hg.) (2011): Aktionsplan 2011 der Bundesregierung zum Schutz von Kindern und Jugendlichen vor sexueller Gewalt und Ausbeutung. Kurzfassung. Online verfügbar unter: https://www.bmfsfj.de/blob/86316/dfd448ac17c06738f8e05aa950e987f7/aktionsplan-2011kurzfassung-barrierefrei-data.pdf (Zugriff: 12.01.18, 15:00 Uhr). 
Bundeszentrale für gesundheitliche Aufklärung (Hg.) (2011): Standards für die Sexualaufklärung in Europa. Rahmenkonzept für politische Entscheidungsträger, Bildungseinrichtungen, Gesundheitsbehörden, Expertinnen und Experten. Köln: Bundeszentrale für gesundheitliche Aufklärung.

(1) Bundeszentrale für gesundheitliche Aufklärung (Hg.) (o. J.): Respekt! Schulen als ideale Orte der Prävention von sexualisierter Gewalt. Eine Handreichung. TRAU DICH! Bundesweite Initiative zur Prävention des sexuellen Kindesmissbrauchs. Köln: Bundeszentrale für gesundheitliche Aufklärung.

(2) Bundeszentrale für gesundheitliche Aufklärung (Hg.) (o. J.): Sexualaufklärung und Familienplanung. Online verfügbar unter: https://www.bzga.de/bot_sexualaufklaerung.html (Zugriff am 23.06.18, 18:45 Uhr).

Bundschuh, Claudia (2010): Sexualisierte Gewalt gegen Kinder in Institutionen. Nationaler und internationaler Forschungsstand. Expertise im Rahmen des Projekts „Sexuelle Gewalt gegen Mädchen und Jungen in Institutionen“. München: Deutsches Jugendinstitut.

Bundszus, Bettina (2011): Bekanntmachung des Bundesministeriums für Bildung und Forschung von Richtlinien zur Förderung von Forschungsvorhaben im Zusammenhang mit sexueller Gewalt gegen Kinder und Jugendliche in pädagogischen Kontexten. Online verfügbar unter: https://www.bmbf.de/foerderungen/bekanntmachung-630.html (Zugriff am 08.09.18, 15:00 Uhr).

Chodan, Wencke / Reis, Olaf / Häßler, Frank (2014): Sexueller Missbrauch von Kindern und Jugendlichen mit Behinderung. In: Fegert, Jörg Michael / Hoffmann, Ulrike / König, Elisa / Niehues, Johanna / Liebhardt, Hubert: Sexueller Missbrauch von Kindern und Jugendlichen: Ein Handbuch zur Prävention und Intervention für Fachkräfte im medizinischen, psychotherapeutischen und pädagogischen Bereich. Wiesbaden: Springer Fachmedien, S. $407-419$.

Dekker, Arne / Henningsen, Anja / Retkowski, Alexandra / Voß, Heinz-Jürgen / Wazlawik, Martin (2015): Curriculum zur Verankerung des Themas „Sexuelle Gewalt in Institutionen“ in universitärer und hochschulischer Lehre. Online verfügbar unter: http://heinzjuergenvoss.de/Curriculum_V6_final.pdf (Zugriff am 22.06.18, 18:05 Uhr).

Deutscher Bundestag (2016): Zum Recht auf sexuelle Selbstbestimmung im Kontext des Internationalen Paktes für wirtschaftliche, soziale und kulturelle Rechte. Online verfügbar unter https://www.bundestag.de/blob/543296/3119a228eadd94ef44dba1ee8c797d13/wd-2-12616-pdf-data.pdf (Zugriff am 23.06.18, 19:45 Uhr). 
Dörr, Margret (2012): Intime Kommunikation in professionellen pädagogischen Beziehungen. In: Thole, Werner / Baader, Meike / Helsper, Werner / Kappeler, Manfred / Leuzinger-Bohleber, Marianne / Reh, Sabine / Sielert, Uwe / Thompson, Christiane (Hg.): Sexualisierte Gewalt, Macht und Pädagogik. Opladen, Berlin, Toronto: Verlag Barbara Budrich, S. $174-185$.

Enders Ursula (2012): „Mistbeet für Täter“. Institutionelle Strukturen und konzeptionelle Mängel, die Missbrauch begünstigen. In: Enders, Ursula (Hg.): Grenzen achten: Schutz vor sexuellem Missbrauch in Institutionen Ein Handbuch für die Praxis. Köln: Kiepenheuer \& Witsch, S. $129-146$.

Enders, Ursula / Romahn, Esther / Villier, Ilka (2012): Klar, diffus, autoritär oder verwahrlost? Institutionelle Strukturen und fachliche Mängel, die den Schutz vor sexuellen Übergriffen und Missbrauch vernachlässigen. In: Enders, Ursula (Hg.): Grenzen achten: Schutz vor sexuellem Missbrauch in Institutionen Ein Handbuch für die Praxis. Köln: Kiepenheuer \& Witsch, S. 147 - 154.

Faulstich - Wieland, Hannelore (2015): Umgang mit Heterogenität und Differenz. In: Huch, Sarah / Lücke, Martin (Hg.): Sexuelle Vielfalt im Handlungsfeld Schule. Konzepte aus Erziehungswissenschaft und Fachdidaktik. Bielefeld: transcript Verlag, S. $49-68$.

Fegert, Jörg Michael / Hoffmann, Ulrike / König, Elisa / Niehues, Johanna / Liebhardt, Hubert (2014): Sexueller Missbrauch von Kindern und Jugendlichen: Ein Handbuch zur Prävention und Intervention für Fachkräfte im medizinischen, psychotherapeutischen und pädagogischen Bereich. Wiesbaden: Springer Fachmedien.

Fegert, Jörg Michael / Wolff, Mechthild (2015): Kompendium „Sexueller Missbrauch in Institutionen“: Entstehungsbedingungen, Prävention und Intervention. Weinheim: Beltz Juventa.

Friebertshäuser, Barbara / Langer, Antje / Pengel, Annedore (Hg.) (2013): Handbuch qualitative Forschungsmethoden in der Erziehungswissenschaft. Weinheim: Beltz Juventa.

Fuchs, Thomas (o. J.): Strafgesetzbuch für das Deutsche Reich vom 15. Mai 1871. Besonderer Teil Dreizehnter Abschnitt. Straftaten gegen die sexuelle Selbstbestimmung. Paragraf 174. Sexueller Mißbrauch von Schutzbefohlenen. Online verfügbar unter: https://lexetius.com/StGB/174/sexueller-missbrauch-von-schutzbefohlenen (Zugriff am 12.08.18, 17:30 Uhr).

Glammeier, Sandra (2018): Sexuelle Gewalt und Schule. In: Wazlawik, Martin / Voß, HeinzJürgen / Retkowski, Alexandra / Henningsen, Anja / Dekker, Arne (Hg.): Sexuelle Gewalt in pädagogischen Kontexten. Aktuelle Forschungen und Reflexionen. Springer Fachmedien: Wiesbaden, S. $197-209$. 
Glammeier, Sandra (2016): Nicht-intentionale Aspekte im pädagogischen Handeln von Lehrkräften im Kontext von sexuellen Übergriffen. In: Mahs, Claudia / Rendtorff, Barbara / Rieske, Thomas Viola (Hg.): Erziehung, Gewalt, Sexualität. Zum Verhältnis von Geschlecht und Gewalt in Erziehung und Bildung. Opladen, Berlin, Toronto: Verlag Barbara Budrich. S. $131-146$.

Hagemann-White, Carol / Herwartz-Emden, Leonie / Hummel, Matthias (2012): Gewalt durch PädagogInnen: Empirische Befunde und Erklärungsansätze. In: In: Thole, Werner / Baader, Meike / Helsper, Werner / Kappeler, Manfred / Leuzinger-Bohleber, Marianne / Reh, Sabine / Sielert, Uwe / Thompson, Christiane (Hg.): Sexualisierte Gewalt, Macht und Pädagogik. Opladen, Berlin, Toronto: Verlag Barbara Budrich, S. 223 - 237.

Hartmann, Jutta (2015): Geschlechtliche und sexuelle Vielfalt im Kontext von Schule und Hochschule. Normativität und Ambivalenz als zentrale Herausforderungen einer Pädagogik vielfältiger Lebensweisen. In: Huch, Sarah / Lücke, Martin (Hg.): Sexuelle Vielfalt im Handlungsfeld Schule. Konzepte aus Erziehungswissenschaft und Fachdidaktik. Bielefeld: transcript Verlag, S. $27-48$.

Heinzel, Friederik / Prengel, Annedore (2018): Sexualisierte Gewalt und Schulen. In: Retkowski, Alexandra / Treibel, Angelika / Tuider, Elisabeth (Hg.) (2018): Handbuch Sexualisierte Gewalt und pädagogische Kontexte. Weinheim und Basel: Beltz Juventa, 415 423.

Helmer, Martin / Muck, Christoph (2014): Präventive Haltung und Arbeit in der schulischen Prävention zum Thema sexualisierte Gewalt. In: Mosser, Peter / Lenz, Hans-Joachim (Hg.): Sexualisierte Gewalt gegen Jungen. Prävention und Intervention. Ein Handbuch für die Praxis. Wiesbaden: Springer Fachmedien, S. 101 - 118.

Helming, Elisabeth / Kindler, Heinz / Langmeyer, Alexandra / Mayer, Marina / Mosser, Peter / Entleitner, Christine / Schutter, Sabine / Wolff, Mechthild (2011): Sexuelle Gewalt gegen Jungen und Mädchen in Institutionen. Abschlussbericht. Hg. von Deutsches Jugendinstitut. Online verfügbar unter: https://www.dji.de/fileadmin/user_upload/bibs/DJIAbschlussbericht_Sexuelle_Gewalt.pdf (Zugriff am 12.08.18, 18:00 Uhr)

Henningsen, Anja / Tuider, Elisabeth / Timmermanns, Stefan (Hg.) (2016): Sexualpädagogik kontrovers. Weinheim und Basel: Beltz Juventa.

Henningsen, Anja (2016): Sexuelle Bildung und Gewaltprävention. Eine systematische Reflexion zur Prävention sexualisierter Gewalt in pädagogischen Kontexten. In: Henningsen, 
Anja / Tuider, Elisabeth / Timmermanns, Stefan (Hg.): Sexualpädagogik kontrovers. Weinheim und Basel: Beltz Juventa, S. 120 - 141.

Hilgers, Andrea (2004): Richtlinien und Lehrpläne zur Sexualerziehung. Eine Analyse der Inhalte, Normen, Werte und Methoden zur Sexualaufklärung in den sechzehn Ländern der Bundesrepublik Deutschland. Eine Expertise im Auftrag der BZgA. Hg. von Bundeszentrale für gesundheitliche Aufklärung. Köln: Bundeszentrale für gesundheitliche Aufklärung.

Hoffmann, Markus (2016): Schulische Sexualerziehung. Deutungsmuster von Lehrenden. Opladen, Berlin, Toronto: Verlag Barbara Budrich.

Hölling, Iris / Riesel-Breidenstein, Dagmar / Schlingmann, Thomas (2015): Mädchen und Jungen vor sexueller Gewalt in Institutionen schützen. Handlungsempfehlungen zur Prävention von sexuellem Missbrauch in Institutionen der Jugendhilfe, Kinder- und Jugendfreizeiteinrichtungen, Schulen und Kindertagesbetreuungseinrichtungen. Berlin: Paritätischer Wohlfahrtsverband.

Hopf, Arnulf (2013): Sexualpädagogik in der Ausbildung von Lehrerinnen und Lehrern. In: Schmidt, Renate-Berenike / Sielert, Uwe: Handbuch Sexualpädagogik und sexuelle Bildung. Weinheim: Beltz Juventa, S. 779-786.

Huch, Sarah / Lücke, Martin (Hg.) (2015): Sexuelle Vielfalt im Handlungsfeld Schule. Konzepte aus Erziehungswissenschaft und Fachdidaktik. Bielefeld: transcript Verlag.

Hummrich, Merle / Kramer, Rolf-Torsten (2017): Schulische Sozialisation. Wiesbaden: Springer Fachmedien.

Illichmann, Jutta (2017): Bekanntmachung. Richtlinien zur Förderung eines Metavorhabens im Rahmen der Förderlinie "Forschung zu sexualisierter Gewalt gegen Kinder und Jugendliche in pädagogischen Kontexten". Online verfügbar unter: https://www.bmbf.de/foerderungen/bekanntmachung-1344.html (Zugriff 21.06.18, 10:00 Uhr). Institut für Angewandte Sexualwissenschaften (2018): „Das ist eben das Dilemma“ Konfrontation mit sexualisierter Gewalt gegen Kinder und Jugendliche im Rahmen der Sozialpädagogischen Familienhilfe (SPFH). Online verfügbar unter: https://www.ifashome.de/wp-

content/uploads/2018/06/Poster_Forschung_Modell_Dilemma_SPFH_17.04.18.pdf (Zugriff am 22.08.18, 17:30 Uhr).

Institut für Angewandte Sexualwissenschaften (2017): Regionale Vernetzung zum Thema „Sexualisierte Gewalt“ - Wozu? Online verfügbar unter: https://www.ifas-home.de/regionalevernetzung/ (Zugriff am 22.06.18, 15:00 Uhr). 
Internetredaktion des Bundesministeriums für Familie, Senioren, Frauen und Jugend (Hg.) (2017): Schutz von Kindern und Jugendlichen vor sexueller Gewalt. Online verfügbar unter: https://www.bmfsfj.de/bmfsfj/themen/kinder-und-jugend/kinder-und-jugendschutz/schutz-vorsexueller-gewalt/schutz-von-kindern-und-jugendlichen-vor-sexueller-gewalt/83904 (Zugriff am 13.01.18, 13:20 Uhr).

Kavemann, Barbara / Graf-van Kesteren, Annemarie / Rothkegel, Sibylle / Nagel, Bianca (2016): Erinnern, Schweigen, Sprechen nach sexueller Gewalt in der Kindheit. Ergebnisse einer Interviewstudie mit Frauen und Männern, die als Kind sexuelle Gewalt erlebt haben. Wiesbaden: Springer Fachmedien.

Kettritz, Torsten (2017): „Täter“ und auch „Opfer“ Jungen und junge Männer mit sexuell übergriffigem Verhalten. In: Stiftung Männergesundheit (Hg.): Sexualität von Männern. Dritter Deutscher Männergesundheitsbericht. Gießen: Psychosozial-Verlag, S 375 - 384.

Kindler, Heinz (2014): Sexuelle Übergriffe in Schulen. In: Willems, Helmut / Ferring, Dieter (Hg.): Macht und Missbrauch in Institutionen. Interdisziplinäre Perspektiven auf institutionelle Kontexte und Strategien der Prävention. Wiesbaden: Springer Fachmedien, S. 111 - 132.

Kollender, Pia (2015): Sexuelle Bildung in der universitären Lehramtsausbildung in NRW - eine quantitative Analyse der Vermittlung von Kompetenzen sexueller Bildung. Masterarbeit an der Hochschule Merseburg.

Krolzik-Matthei, Katja / Linke, Torsten / Voß, Heinz-Jürgen (2018): Interviews zu Sexualität und Geschlecht mit Fachkräften in der Sozialen Arbeit - Die Bedeutung einer reflektierten Haltung für das professionelle Handeln am Beispiel des Umgangs mit Macht und Diskriminierungserfahrungen im Arbeitskontext. In: Wazlawik, Martin / Voß, Heinz-Jürgen / Retkowski, Alexandra / Henningsen, Anja / Dekker, Arne (Hg.) (2018): Sexuelle Gewalt in pädagogischen Kontexten. Aktuelle Forschungen und Reflexionen. Springer Fachmedien: Wiesbaden, S. $247-259$.

Krolzik-Matthei, Katja / Linke, Torsten (2017): Gespräche mit (sozial)pädagogischen Fachkräften. Ressourcen, Kompetenzen, Widersprüche im Umgang mit Sexualität und sexualisierter Gewalt in professionellen Settings. Powerpointpräsentation zum Vortrag am 01.12.2017. DGfPI-Bundestagung in Merseburg. Online verfügbar unter: https://www.dgfpi.de/files/was-wir-tun/fachtagungen/Fachtagungen\%202017/2017-12-01DGfPI_Bundestagung_Vortrag_Krolzik-Matthei_Linke.pdf (Zugriff am 19.01.18, 19:30 Uhr).

Krolzik-Matthei, Katja / Voß, Heinz-Jürgen (2016): Gewalt kommt in den Blick: über aktuelle Forschungen und Debatten. In: Henningsen, Anja / Tuider, Elisabeth / Timmermanns, Stefan (Hg.): Sexualpädagogik kontrovers. Weinheim und Basel: Beltz Juventa, S. 105 - 119. 
Kultusministerkonferenz (2013): Handlungsempfehlungen der Kultusministerkonferenz zur Vorbeugung und Aufarbeitung von sexuellen Missbrauchsfällen und Gewalthandlungen in Schulen und schulnahen Einrichtungen. Online verfügbar unter: https://www.kmk.org/fileadmin/veroeffentlichungen_beschluesse/2010/2010_04_20Handlungsempfehlungen-Vorbeugung-sexueller-Missbrauch_2013.pdf (Zugriff am 14.08.18, 17:00 Uhr).

Lache, Lena (2018): Autismus und sexualisierte Gewalt. In: Retkowski, Alexandra / Treibel, Angelika / Tuider, Elisabeth (Hg.): Handbuch Sexualisierte Gewalt und pädagogische Kontexte. Weinheim und Basel: Beltz Juventa, S. 297 - 305.

Lamnek, Siegfried / Krell, Claudia (2016): Qualitative Sozialforschung: mit Online-Material. Weinheim: Beltz.

Langer, Annette (2015): Der Missbrauchsskandal in der Katholischen Kirche. Online verfügbar unter: http://www.spiegel.de/panorama/chronik-der-missbrauchsskandal-in-der-katholischenkirche-a-1012711.html. (Zugriff 12.01.18, 13:45 Uhr).

Lücke, Martin (2015): Vom „Normalkind“ zu einer Sexualpädagogik der Vielfalt. Homosexualitäten in den Bildungswissenschaften. In: Huch, Sarah / Lücke, Martin (Hg.): Sexuelle Vielfalt im Handlungsfeld Schule. Konzepte aus Erziehungswissenschaft und Fachdidaktik. Bielefeld: transcript Verlag, S. 13-26.

Mahs, Claudia / Rendtorff, Barbara / Rieske, Thomas Viola (Hg.) (2016): Erziehung, Gewalt, Sexualität. Zum Verhältnis von Geschlecht und Gewalt in Erziehung und Bildung. Opladen, Berlin, Toronto: Verlag Barbara Budrich.

Maschke, Sabine / Stecher, Ludwig (2018): SPEAK! Die Studie. „Sexualisierte Gewalt in der Erfahrung Jugendlichen. Erweiterungsstudie Förderschulen. Kurzbericht. Online verfügbar unter: http://www.speak-studie.de/Kurzbericht\%20Speak_Förderschule_2018-04-12.pdf (Zugriff 30.04.19, 10:30 Uhr).

Maschke, Sabine / Stecher, Ludwig (2017): SPEAK! Die Studie. „Sexualisierte Gewalt in der Erfahrung Jugendlicher. Öffentlicher Kurzbericht. Online verfügbar unter: http://www.speakstudie.de/assets/uploads/kurzberichte/201706_Kurzbericht-Speak.pdf (Zugriff 30.04.19, 10:30 Uhr).

Milhoffer, Petra (2013): Sexualpädagogik in der Grundschule. Schmidt, Renate-Berenike / Sielert, Uwe: Handbuch Sexualpädagogik und sexuelle Bildung. Weinheim: Beltz Juventa, S. 582-592. 
Mayring, Philipp (2015): Qualitative Inhaltsanalyse. Grundlagen und Techniken. Weinheim: Beltz.

Mosser, Peter / Lenz, Hans-Joachim (Hg.) (2014): Sexualisierte Gewalt gegen Jungen. Prävention und Intervention. Ein Handbuch für die Praxis. Wiesbaden: Springer Fachmedien.

Münder, Johannes / Kavemann, Barbara (2010): Sexuelle Übergriffe in der Schule. Leitfaden für Schulleitungen, Schulaufsicht und Kollegen zur Währung des sexuellen Selbstbestimmungsrechts von Schülerinnen und Schülern. Kiel: PETZE-Institut für Gewaltprävention.

(1) ohne Autor (o. J.): Sexuelle Bildung angehender Lehrerinnen und Lehrer für Sekundarstufe I HRGE. Sexuelle Bildung als historisch gewachsene und gegenwärtige Herausforderung im Lehrberuf. Online verfügbar unter https://www.erziehungswissenschaft.uniwuppertal.de/forschung/allgemeine-erziehungswissenschafttheorie-der-

bildung/forschungsprojekte/sexuelle-bildung-angehender-lehrerinnen-und-lehrer-fuer-diesekundarstufe-i-hrge/historische-und-gesellschaftliche-problembeschreibung.html (Zugriff am 20.06.18 um 11:30 Uhr).

(2) ohne Autor (o. J.): Das Wissenschaftssystem. Qualitätsoffensive Lehrerfortbildung. Online verfügbar unter: https://www.bmbf.de/de/qualitaetsoffensive-lehrerbildung-525.html (Zugriff am 20.06.18, 10:10 Uhr).

(3) ohne Autor (2016): Hohe Dunkelziffer, hilflose Lehrer. Online verfügbar unter: http://www.spiegel.de/lebenundlernen/schule/schule-gegen-sexuelle-gewalt-initiative-zumschutz-von-kindern-a-1112062.html (Zugriff am 20.10.18, 17:45 Uhr).

Poelchau, Heinz-Werner / Briken, Peer / Wazlawik, Martin / Bauer, Ullrich / Fegert, Jörg Michael / Kavemann, Barbara. (2015): Bonner Ethik-Erklärung - Empfehlungen für die Forschung zu sexueller Gewalt in pädagogischen Kontexten. Online verfügbar unter: https://www.bmbf.de/files/Ethikerklaerung\%281\%29.pdf (Zugriff am 13.01.18, 18:30 Uhr).

Pohl, Frank G. (o. J.): Aktiv in den Schulen - Die Projektschulen in NRW. Online verfügbar unter: http://www.schule-der-vielfalt.de/schulen_projektschulen.htm (Zugriff am 25.08.18, 17:00 Uhr).

Pressestelle des Hessischen Kultusministeriums (2017): SPEAK!-Studie: „Hauptrisiko sind gleichaltrige Jugendliche“. Untersuchung zu Erfahrungen von Jugendlichen mit sexualisierter Gewalt. Online verfügbar unter: https://kultusministerium.hessen.de/pressearchiv/pressemitteilung/speak-studie-hauptrisikosind-gleichaltrige-jugendliche (Zugriff am 27.08.18, 19:00 Uhr). 
Rabold, Susann / Baier, Dirk (2008): Gewalt und andere Formen abweichenden Verhaltens in Förderschulen für Lernbehinderte. Erschienen in: Zeitschrift für Pädagogik, 54 (1), 118 - 141.

Reichertz, Jo (2016): Qualitative und interpretative Sozialforschung. Eine Einladung. Wiesbaden: Springer Fachmedien.

Retkowski, Alexandra / Treibel, Angelika / Tuider, Elisabeth (Hg.) (2018): Handbuch Sexualisierte Gewalt und pädagogische Kontexte. Weinheim und Basel: Beltz Juventa.

Ricken, Norbert (2012): Macht, Gewalt und Sexualität in pädagogischen Beziehungen. In: Thole, Werner / Baader, Meike / Helsper, Werner / Kappeler, Manfred / Leuzinger-Bohleber, Marianne / Reh, Sabine / Sielert, Uwe / Thompson, Christiane (Hg.): Sexualisierte Gewalt, Macht und Pädagogik. Opladen, Berlin, Toronto: Verlag Barbara Budrich, S. 103 - 117.

Riesau, Petra (2018): Verständnis von Prävention. Online verfügbar unter: http://www.schulische-praevention.de/wissensbereich-sexualisiertegewalt/praevention/begriffe/ (Zugriff am 01.09.18, 14:40 Uhr).

Rörig, Johannes-Wilhelm (o. J.): Schule gegen sexuelle Gewalt. Willkommen. Online verfügbar unter: https://www.schule-gegen-sexuelle-gewalt.de/home/ (Zugriff am 01.09.18, 14:45 Uhr).

Runder Tisch (2011/12): Abschlussbericht Runder Tisch Sexueller Kindesmissbrauch in Abhängigkeits- und Machtverhältnissen in privaten und öffentlichen Einrichtungen und im familiären Bereich. Berlin: BMJ, BMFSFJ, BMBF. Online verfügbar unter: http://www.bmjv.de/SharedDocs/Downloads/DE/Fachinformationen/Abschlussbericht_RTKM. pdf?_blob=publicationFile (Zugriff am 13.01.18, 18:35 Uhr).

Sager, Christin (2015): Das aufgeklärte Kind. Zur Geschichte der bundesrepublikanischen Sexualaufklärung (1950 - 2010). Bielefeld: transcript Verlag.

Schmid, Conny (2012): Sexuelle Übergriffe an Kindern und Jugendlichen in der Schweiz. Formen, Verbreitung, Tatumstände. Hg. v. UBS Optimus Foundation. Online verfügbar unter http://www.optimusstudy.org/fileadmin/user_upload/documents/Booklet_Schweiz/Optimus_St udie_Broschuere_2012_d.pdf (Zugriff am 19.09.2018).

Schmidt, Renate-Berenike (2013): Sexualerziehung in der Sekundarstufe I. In: Schmidt, Renate-Berenike / Sielert, Uwe: Handbuch Sexualpädagogik und sexuelle Bildung. Weinheim: Beltz Juventa, S. 593-600.

Schmidt, Renate-Berenike / Schetsche, Michael (2013): Intime Kommunikation in der Schule. In: Schmidt, Renate-Berenike / Sielert, Uwe: Handbuch Sexualpädagogik und sexuelle Bildung. Weinheim: Beltz Juventa, S. 601-609. 
Schmidt, Renate-Berenike / Sielert, Uwe (Hg.) (2013): Handbuch Sexualpädagogik und sexuelle Bildung. Weinheim: Beltz Juventa.

Schmidt, Renate-Berenike (2014): Schule als Ort sexueller Sozialisation. In: Hagedorn, Jörg (Hg.): Jugend, Schule, Identität. Selbstwerdung und Identitätskonstrukt im Kontext Schule. Wiesbaden: Springer Fachmedien, S. 249 - 264.

Schubarth, Wilfried / Ulbricht, Juliane (2012): Sexualisierte Gewalt an Schulen. In: Thole, Werner / Baader, Meike / Helsper, Werner / Kappeler, Manfred / Leuzinger-Bohleber, Marianne / Reh, Sabine / Sielert, Uwe / Thompson, Christiane (Hg.): Sexualisierte Gewalt, Macht und Pädagogik. Opladen, Berlin, Toronto: Verlag Barbara Budrich, S. 238 - 248.

(1) Sekretariat der Ständigen Konferenz der Kultusminister der Länder in der Bundesrepublik Deutschland (o. J.): Aufgaben der Kultusministerkonferenz. Online verfügbar unter: https://www.kmk.org/kmk/aufgaben.html (Zugriff am 25.08.18, 15:30 Uhr).

(2) Sekretariat der Ständigen Konferenz der Kultusminister der Länder in der Bundesrepublik Deutschland (o. J.): Zu Geschichte der Kultusministerkonferenz 1948 - 1998. Online verfügbar unter: https://www.kmk.org/kmk/aufgaben/geschichte-der-kmk.html (Zugriff am 25.08.18, 15:15 Uhr).

Siedenbiedel, Catrin (2018): Prävention von sexualisierter Gewalt im Kontext inklusiver Bildung. In: Retkowski, Alexandra / Treibel, Angelika / Tuider, Elisabeth (Hg.) (2018): Handbuch Sexualisierte Gewalt und pädagogische Kontexte. Weinheim und Basel: Beltz Juventa, S. $552-560$.

Sielert, Uwe (2016): Didaktik der Sexualpädagogik: historische und systematische Kontroversen. In: Henningsen, Anja / Tuider, Elisabeth / Timmermanns, Stefan (Hg.): Sexualpädagogik kontrovers. Weinheim und Basel: Beltz Juventa, S. $69-88$.

(1) Sielert, Uwe (2015): Einführung in die Sexualpädagogik. Weinheim, Basel: Beltz Juventa.

(2) Sielert, Uwe (2015): Sexuelle Vielfalt als Thema der Sexualpädagogik. In: Huch, Sarah / Lücke, Martin (Hg.): Sexuelle Vielfalt im Handlungsfeld Schule. Konzepte aus Erziehungswissenschaft und Fachdidaktik. Bielefeld: transcript Verlag, S. 93-109.

(1) Sielert, Uwe (2013): Sexualpädagogik und Sexualerziehung in Theorie und Praxis. In: Schmidt, Renate-Berenike / Sielert, Uwe (Hg.): Handbuch Sexualpädagogik und sexuelle Bildung. Weinheim: Beltz Juventa, S. $41-54$.

(2) Sielert, Uwe (2013): Professionalisierung in der Sexualpädagogik. In: Schmidt, RenateBerenike / Sielert, Uwe: Handbuch Sexualpädagogik und sexuelle Bildung. Weinheim: Beltz Juventa, S. 757-767. 
Sielert, Uwe (2012): Sexual- und Erziehungswissenschaft. In: Thole, Werner / Baader, Meike / Helsper, Werner / Kappeler, Manfred / Leuzinger-Bohleber, Marianne / Reh, Sabine / Sielert, Uwe / Thompson, Christiane (Hg.): Sexualisierte Gewalt, Macht und Pädagogik. Opladen, Berlin, Toronto: Verlag Barbara Budrich, S. $208-221$.

Sielert, Uwe (2011): Expertise zum Thema „Sexualerziehung in Grundschulen“. Kiel. Online verfügbar unter: https://www.sozialpaedagogik.uni-kiel.de/de/downloads/expertiselangfassung-1_2015 (Zugriff am 15.01.18, 19:00 Uhr).

Silkenbeumer, Mirja (2016): Gewalt und Geschlecht in der Schule: Gewalthandlungen von Schülerinnen und Schülern im Spiegel geschlechtertheoretischer Perspektiven. In: Mahs, Claudia / Rendtorff, Barbara / Rieske, Thomas Viola (Hg.): Erziehung, Gewalt, Sexualität. Zum Verhältnis von Geschlecht und Gewalt in Erziehung und Bildung. Opladen, Berlin, Toronto: Verlag Barbara Budrich, S. $57-78$.

Statistisches Bundesamt (2017): Kinder- und Jugendhilfe in Deutschland. Erzieherische Hilfen und sonstige Leistungen. Online verfügbar unter: https://www.destatis.de/DE/ZahlenFakten/GesellschaftStaat/Soziales/Sozialleistungen/Kinde rJugendhilfe/Tabellen/ErzieherischeHilfen.html (Zugriff am 13.01.18, 20:00 Uhr).

Stiftung Männergesundheit (Hg.) (2017): Sexualität von Männern. Dritter Deutscher Männergesundheitsbericht. Gießen: Psychosozial-Verlag.

Thole, Werner / Baader, Meike / Helsper, Werner / Kappeler, Manfred / Leuzinger-Bohleber, Marianne / Reh, Sabine / Sielert, Uwe / Thompson, Christiane (Hg.) (2012): Sexualisierte Gewalt, Macht und Pädagogik. Opladen, Berlin, Toronto: Verlag Barbara Budrich.

Tuider, Elisabeth (2017): Sichtweisen auf sexualisierte Gewalt und sexualisierte Grenzüberschreitungen unter Jugendlichen. In: Stiftung Männergesundheit (Hg.): Sexualität von Männern. Dritter Deutscher Männergesundheitsbericht. Gießen: Psychosozial-Verlag, S. $361-374$.

Tuider, Elisabeth / Müller, Mario / Timmermanns, Stefan / Bruns-Bachmann, Petra / Koppermann, Carola (2012): Sexualpädagogik der Vielfalt. Praxismethoden zu Identitäten, Beziehungen, Körper und Prävention für Schule und Jugendarbeit. Weinheim und München: Beltz Juventa.

UBS Optimus Foundation (Hg.) (2012): Sexuelle Übergriffe an Kindern und Jugendlichen in der Schweiz. Formen, Verbreitung, Tatumstände. Zürich.

Unabhängiger Beauftragter für Fragen des sexuellen Kindesmissbrauchs (2017): Fakten und Zahlen zu sexueller Gewalt an Kindern und Jugendlichen. Definition von sexueller Gewalt an 
Mädchen und Jungen. Online verfügbar unter: https://beauftragtermissbrauch.de/fileadmin/Content/pdf/Pressemitteilungen/2017/05_Oktober/6_Fact_Sheet_Z ahlen_Ausmaß_sex_Gewalt.pdf (Zugriff am 12.05.18, 20:00 Uhr).

Unabhängiger Beauftragter für Fragen des sexuellen Kindesmissbrauchs (Hg.) (2012): Handbuch Schutzkonzepte sexueller Missbrauch. Befragungen zum Umsetzungsstand der Empfehlungen des Runden Tisches „Sexueller Kindesmissbrauch“. Bericht mit Praxisbeispielen zum Monitoring 2012 - 2013. Berlin: Geschäftsstelle des Unabhängigen Beauftragen für Fragen des sexuellen Kindesmissbrauchs.

UNICEF Deutschland (o. J.): Konvention über die Rechte des Kindes. Online verfügbar unter: https://www.unicef.de/blob/9364/a1bbed70474053cc61d1c64d4f82d604/d0006-

kinderkonvention-pdf-data.pdf (Zugriff am 20.06.18, 11:30 Uhr).

Valtl, Karlheinz (2013): Sexuelle Bildung: Neues Paradigma einer Sexualpädagogik für alle Lebensalter. In: Schmidt, Renate-Berenike / Sielert, Uwe $(\mathrm{Hg})$ : Handbuch Sexualpädagogik und sexuelle Bildung. Weinheim, Basel, München: Beltz Juventa, S. 125-140.

von Unger (2014): Forschungsethik in der qualitativen Forschung: Grundsätze, Debatten und offene Fragen. In: von Unger, Hella / Narimani, Petra / M'Bayo, Rosaline (Hg.): Forschungsethik in der qualitativen Sozialforschung. Reflexivität, Perspektiven, Positionen. Wiesbaden: Springer Fachmedien, S. 15 - 39.

von Unger, Hella / Narimani, Petra / M'Bayo, Rosaline (Hg.) (2014): Forschungsethik in der qualitativen Sozialforschung. Reflexivität, Perspektiven, Positionen. Wiesbaden: Springer Fachmedien.

Vogelsang, Verena / Riesau, Petra (2018): Qualitätsstandards. Online verfügbar unter: http://www.schulische-praevention.de/hilfe-fuer-paedagogische-

fachkraefte/projekte/qualitaetsstandards/ (Zugriff am 01.09.18, 14:15 Uhr).

Voß, Heinz-Jürgen (2016): Forschungsprofessur "Sexualwissenschaft und sexuelle Bildung". In: Bundesministerium für Bildung und Forschung (Hg.): Sexuelle Gewalt gegen Kinder und Jugendliche in pädagogischen Kontexten: Forschung zu Prävention und Schutzkonzepten, S. 54-55.

Voß, Heinz-Jürgen (2015f): Sexualisierte Gewalt - Überblick über aktuelle Forschungen. Newsletter des Sexualwissenschaftlichen interdisziplinären Nachwuchses, 1/2015.

Voß, Heinz-Jürgen / Younes, Anna-Esther / Krolzik-Matthei, Katja (2014): Schutz von Kindern und Jugendlichen vor sexueller Traumatisierung. Forschungsdesign. Merseburg. Wazlawik, Martin / Voß, Heinz-Jürgen / Retkowski, Alexandra / Henningsen, Anja / Dekker, Arne (Hg.) 
(2018): Sexuelle Gewalt in pädagogischen Kontexten. Aktuelle Forschungen und Reflexionen. Springer Fachmedien: Wiesbaden.

Weller, Konrad (2013): Sexualitätsbezogene Ausbildung im Hochschulstudium - das Merseburger Beispiel. In: Schmidt, Renate-Berenike / Sielert, Uwe: Handbuch Sexualpädagogik und sexuelle Bildung. Weinheim: Beltz Juventa, S. 768-778.

(1) Wienholz, Sabine / Retznik, Laura (2018): „Teilhabechancen an sexueller Bildung von Jugendlichen und jungen Erwachsenen mit kognitiven Beeinträchtigungen in Sachsen“. Präsentation zum Vortrag am 18.01.2018. Fachtagung „Zwischen Tabu und Normalität“, Leben mit Handicap e.V.. Leipzig.

(2) Wienholz, Sabine / Retznik, Laura (2018): „Sexuelle Bildung und kognitive Beeinträchtigungen - für Multiplikatoren“. Präsentation zum Workshop am 18.01.2018. Fachtagung „Zwischen Tabu und Normalität“, Leben mit Handicap e.V.. Leipzig.

Willems, Helmut / Ferring, Dieter (Hg.) (2014): Macht und Missbrauch in Institutionen. Interdisziplinäre Perspektiven auf institutionelle Kontexte und Strategien der Prävention. Wiesbaden: Springer Fachmedien.

Wolff, Mechthild (2014): Missbrauch von Kindern und Jugendlichen in Institutionen. Perspektiven der Prävention durch Schutzkonzepte. In: Willems, Helmut / Ferring, Dieter (Hg.): Macht und Missbrauch in Institutionen. Interdisziplinäre Perspektiven auf institutionelle Kontexte und Strategien der Prävention. Wiesbaden: Springer Fachmedien, S. 151 - 166.

Wiessenthal, Ann (2017): Antisexisitische Awareness. Ein Handbuch. Münster. UNRASTVerlag. 


\section{Anhang}

\section{Transkripte}

Transkripte FS1 - FS6 siehe beiliegendem Datenträger.

\section{Kurzprotokolle}

Kurzprotokolle FS1, FS2, FS3 und FS6 siehe beiliegendem Datenträger.

\section{Interviewleitfaden}

\begin{tabular}{|l|l|l|l|l|l|}
\hline Einleitung & $\begin{array}{l}\text { Personen } \\
\text { / Kultur }\end{array}$ & $\begin{array}{l}\text { Sexualität / } \\
\text { Sexuelle Bildung }\end{array}$ & $\begin{array}{l}\text { Beschwerden / } \\
\text { Grenzverletzung }\end{array}$ & $\begin{array}{l}\text { Kompetenz/ } \\
\text { Qualifikation }\end{array}$ & $\begin{array}{l}\text { ZurPerson/ } \\
\text { Einrichtung }\end{array}$ \\
\hline
\end{tabular}

\section{Einleitung}

Ich begrüße Sie ganz herzlich und bedanke mich für Ihre Bereitschaft, dieses Interview mit mir zu führen. In den Informationen zum Forschungsprojekt, die ich Ihnen eben erläutert und schriftlich vorgelegt habe, haben Sie bereits den Hintergrund und das Anliegen zu diesem Interview erfahren.

(Kurze Information zur eigenen Person: Rolle im Forschungsprojekt)

In unserem Gespräch geht es mir darum, Ihre Erfahrungen und Ihre persönliche Sicht auf die Thematik zu erfahren.

Ich weise Sie an dieser Stelle noch einmal darauf hin, dass die Teilnahme am Interview freiwillig ist und Sie jederzeit die Möglichkeit haben, dass Interview zu unterbrechen oder abzubrechen.

Bevor ich Ihnen einige Fragen stelle, bitte ich Sie, mir erst einmal zu erzählen inwiefern Sie in Ihrer Arbeit mit dem Thema Sexualität zu tun haben. Gehen Sie dabei auf alles ein, was Ihnen in diesem Zusammenhang einfällt. 


\begin{tabular}{|l|l|l|l|l|l|}
\hline Einleitung & $\begin{array}{l}\text { Personen/ } \\
\text { Kultur }\end{array}$ & $\begin{array}{l}\text { Sexualität / } \\
\text { Sexuelle Bildung }\end{array}$ & $\begin{array}{l}\text { Beschwerden / } \\
\text { Grenzverletzung }\end{array}$ & $\begin{array}{l}\text { Kompetenz/ } \\
\text { Qualifikation }\end{array}$ & $\begin{array}{l}\text { ZurPerson/ } \\
\text { Einrichtung }\end{array}$ \\
\hline
\end{tabular}

\begin{tabular}{l|l} 
II. Zielgruppe & Notizen
\end{tabular}

(1) Zusammensetzung / Merkmale der Zielgruppe /

der Schüler_innenschaft

\begin{tabular}{l|l} 
III. Mitarbeiter_innen & Notizen
\end{tabular}

(1) Zusammensetzung/Merkmaledes Teams/des Kollegiums

\begin{tabular}{l|l} 
IV. Kultur und Kommunikation & Notizen
\end{tabular}

(1) Klima und Atmosphäre in der Einrichtung / der Schule

Nachfragenzu den Umgangsweiseninnerhalbund zwischen verschiedenen Personengruppen der Einrichtung 


\begin{tabular}{|c|c|c|c|c|c|}
\hline Einleitung & $\begin{array}{l}\text { Personen/ } \\
\text { Kultur }\end{array}$ & $\begin{array}{l}\text { Sexualität / } \\
\text { SexuelleBildung }\end{array}$ & $\begin{array}{l}\text { Beschwerden / } \\
\text { Grenzverletzung }\end{array}$ & $\begin{array}{l}\text { Kompetenz/ } \\
\text { Qualifikation }\end{array}$ & $\begin{array}{l}\text { ZurPerson/ } \\
\text { Einrichtung }\end{array}$ \\
\hline
\end{tabular}

\begin{tabular}{l|l} 
VII. Sexualität & Notizen
\end{tabular}

(1) Häufig auftretende Themen

(2) Personen, die Thema vorwiegend aufrufen

\begin{tabular}{l|l} 
VIII. SexuelleBildung & Notizen
\end{tabular}

Bei Verständnisfragen: keine Definition anbieten

(1) Angebote sexueller Bildung

(2) Personen, die Aufgaben im Kontext sexueller Bildung wahrnehmen 


\begin{tabular}{l|l|}
\hline V. Beschwerden & Notizen \\
\hline (1) Möglichkeitenfür Jugendliche, sich anzuvertrauen & \\
(2) Umgang mitBeschwerden & \\
(3) Bekanntmachung von Möglichkeiten in der & \\
Einrichtung / Schule &
\end{tabular}

VI. Grenzverletzung

Notizen

(1) Eigene Definition von Grenzverletzung

(2) Fälle vonGrenzverletzung

(3) Umgang mit Fällen

(4) Maßnahmen zur Verhinderung

(5) Nicht-sexuelle Grenzverletzungen 


\begin{tabular}{|c|c|c|c|c|c|}
\hline Einleitung & $\begin{array}{l}\text { Personen/ } \\
\text { Kultur }\end{array}$ & $\begin{array}{l}\text { Sexualität / } \\
\text { Sexuelle Bildung }\end{array}$ & $\begin{array}{l}\text { Beschwerden / } \\
\text { Grenzverletzung }\end{array}$ & $\begin{array}{l}\text { Kompetenzl } \\
\text { Qualifikation }\end{array}$ & $\begin{array}{l}\text { ZurPerson/ } \\
\text { Einrichtung }\end{array}$ \\
\hline
\end{tabular}

IX. Kompetenz

Notizen

(1) Eigene Kompetenzen

(2) Bedeutung des Themas in Ausbildung

(3) Qualifikation von Kolleg_innen

X. Fort- und Weiterbildung

Notizen

(1) BekannteFort-undWeiterbildungsangebote

(2) Bereits in Anspruch genommene Angebote

a. Wichtigste Inhalte

b. Einschätzungüber Aufbau, Inhalteund Methoden

(3) gewünschteFort-undWeiterbildungsangebote im zu Sexualität und Grenzverletzung

(4) Wunsch an Strukturund Organisation von Angeboten 


\begin{tabular}{|l|l|l|l|l|l|}
\hline Einleitung & $\begin{array}{l}\text { Personen/ } \\
\text { Kultur }\end{array}$ & $\begin{array}{l}\text { Sexualität / } \\
\text { Sexuelle Bildung }\end{array}$ & $\begin{array}{l}\text { Beschwerden / } \\
\text { Grenzverletzung }\end{array}$ & $\begin{array}{l}\text { Kompetenz/ } \\
\text { Qualifikation }\end{array}$ & $\begin{array}{l}\text { Zur Person/ } \\
\text { Einrichtung }\end{array}$ \\
\hline
\end{tabular}

\section{Zur Person}

1. Jetzt sind wir fast am Ende. Zuvor habe ich noch zwei Fragen zu Ihrer Person.

(1) Alter

(2) Geschlechtszuordnung ja/nein

Wenn ja:

a) Welchem Geschlecht ordnen Sie sich zu?

(3) eigene Beschäftigung mit dem Thema des Interviews

\section{XII.Zur Einrichtung}

(1) Anzahl Mitarbeiter_innen

(2) Anzahl Klient_innen

(3) Größe des Trägers (Einrichtung einziges Projekt oder mehrere Projekte)

(4) Tätigkeitsbereich/Einzugsbereich der Einrichtung (städtischer oder ländlicher Raum)

\section{Abschluss}

(1) Ergänzungen von Interviewter

(2) Erleben des Interviews

(3) Dank und Abschied! 


\section{Informationen zum Forschungsprojekt}

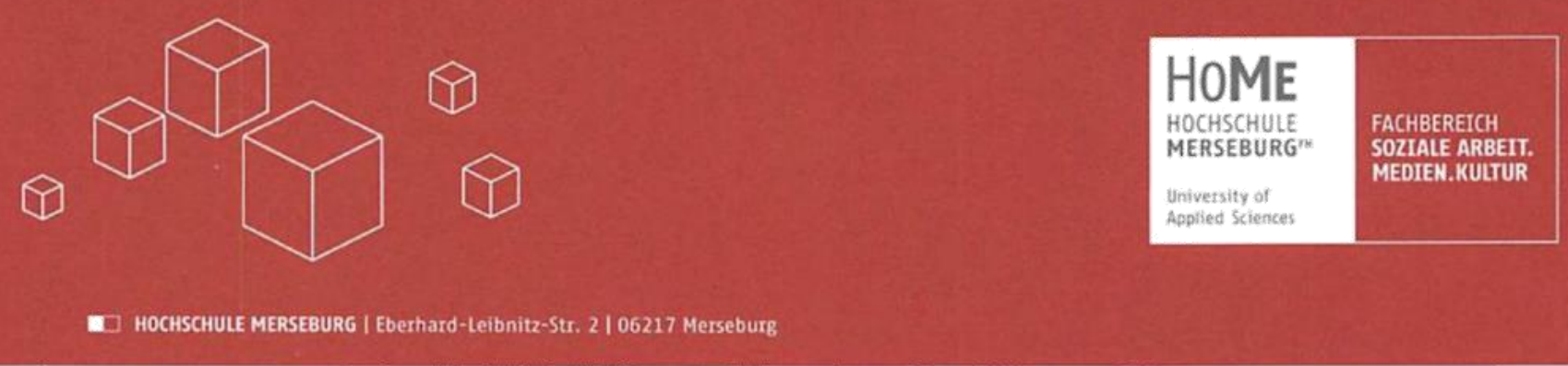

Sehr geehrte_r Interviewteilnehmer_in!

\section{Informationen zum Forschungsprojekt „Schutz von Kindern und Jugendlichen vor sexueller Traumatisierung“}

Wir informieren Sie über das Forschungsprojekt, für das wir Sie gern interviewen möchten, und über unser Vorgehen. Der Datenschutz verlangt Ihre ausdrückliche und informierte Einwilligung dazu, dass wir das Interview speichern und auswerten.

Die verantwortliche Leitung des Projekts liegt bei Prof. Dr. HeinzJürgen Voß, Professor für Sexuelle Bildung und Sexualwissenschaft an der Hochschule Merseburg.

In dem Forschungsprojekt möchten wir herausfinden, welche Kompetenzen und Qualifizierungsbedarfe pädagogische Fachkräfte haben, um in ihren Einrichtungen Grenzverletzungen und sexuelle Übergriffe zu vermeiden, zu verhindern und Kinder und Jugendliche bei der Verarbeitung erlebter Grenzverletzungen zu unterstützen und zu begleiten. Um das herauszufinden, befragen wir sowohl die pädagogischen Fachkräfte als auch die Kinder und Jugendlichen in den Einrichtungen nach ihren Erfahrungen und Wahrnehmungen. Ein weiterer Schwerpunkt der Erhebung ist, auch sensibel gegenüber Mehrfachdiskriminierung (u.a. Geschlecht, Sexualität, Behinderung, Klassenzugehörigkeit und Ethnizität) zu sein und diese mit zu erheben. Unser Ziel ist es, auf Basis dieser umfangreichen Erkenntnisse geeignete und passgenaue Fortbildungsbausteine für pädagogische Fachkräfte weiterzuentwickeln.

Die Forschung wird durch die Förderlinie „Sexualisierte Gewalt in pädagogischen Einrichtungen“ des Bundesministeriums für Bildung und Forschung finanziert.
Forschungsprojekt Schutz von Kindern und Jugendlichen vor sexueller Traumatisierung

Institut für Angewandte Sexualwissenschaft (IfAS)

Fachbereich Soziale Arbeit. Medien. Kultur

\section{ANSCHRIFT}

Hochschule Merseburg (FH)

Geusaer Straße

06217 Merseburg / Deutschland

\section{TELEFON}

+49 3461 46-2208

\section{TELEFAX}

+49 3461 46-2205

\section{E-MAIL/INTERNET}

heinz-juergen.voss@hs-merseburg.de katja.krolzik-matthei@hs-merseburg.de torsten.linke@hs-merseburg.de

$\underline{\text { www.hs-merseburg.de }}$ 
Die Durchführung der Studie geschieht auf Grundlage des Bundesdatenschutzgesetzes und wurde durch eine Ethikkommission genehmigt. Die Interviewenden unterliegen der Schweigepflicht und

Wir sichern Ihnen folgendes Verfahren zu, damit Ihre Angaben nicht mit Ihrer Person in Verbindung gebracht werden können:

- $\quad$ Wir gehen sorgfältig mit dem Erzählten um: Wir nehmen das Gespräch auf Tonträger auf. Das gesprochene Wort wird abgetippt und anschließend gelöscht.

- $\quad$ Wir anonymisieren, d.h. wir verändern alle Personen-, Orts-, Straßen- und Einrichtungsnamen. Einrichtungen werden lediglich in drei Kategorien aufgeführt: Schule/Schultyp, Sozialpädagogische Familienhilfe und Allgemeiner Sozialdienst, Beratungsstelle, außerschulische Jugend- und Erwachsenenbildung, Einrichtungen der offenen Kinderund Jugendarbeit. Berufe werden ebenfalls gruppiert: Lehrer_in/Schulleitung oder sozialpädagogische Fachkraft.

- $\quad$ Die Abschrift Ihres Interviews sowie die Einwilligungserklärung werden mit demselben Code versehen. In einem Dokument, dass nur der Projektleitung zugänglich ist, wird eine Übersicht geführt, welches Interview mit welchem Code versehen wurde.

- $\quad$ Ihr Name wird am Ende des Projektes in unseren Unterlagen gelöscht, sodass lediglich das anonymisierte Transkript (abgetipptes Interview) existiert. Die von Ihnen unterschriebene Erklärung zur Einwilligung in die Auswertung wird in einem gesonderten Ordner an einer gesicherten und nur der Projektleitung zugänglichen Stelle aufbewahrt. Sie dient lediglich dazu, bei einer Überprüfung durch den_die Datenschutzbeauftragte_n nachweisen zu können, dass Sie mit der Auswertung einverstanden sind. Sie kann mit Ihrem Interview nur durch die Projektleitung in Verbindung gebracht werden.

- $\quad$ Die Abschrift wird nicht veröffentlicht und ist nur projektintern für die Auswertung zugänglich. Die anonymisierte Abschrift wird ausschließlich von den Mitarbeiter_innen des Projektes gelesen, die ebenfalls der Schweigepflicht unterliegen. In die Veröffentlichungen gehen einzelne Zitate ein.

Selbstverständlich wird darin nicht erkennbar sein, von welcher Person diese stammen.

Die Datenschutzbestimmungen verlangen auch, dass wir Sie noch einmal ausdrücklich darauf hinweisen, dass aus einer Nichtteilnahme keine Nachteile entstehen. Sie können Antworten bei einzelnen 
Fragen verweigern. Auch die Einwilligung ist freiwillig und kann jederzeit von Ihnen widerrufen und die Löschung des Interviews verlangt werden. Ein Abbruch des Interviews auf Ihren Wunsch ist zu jedem Zeitpunkt möglich. Es ergeben sich daraus für Sie keinerlei negative Konsequenzen.

Wir bedanken uns für Ihre Bereitschaft, uns Auskunft zu geben, und hoffen, unsere wissenschaftliche Arbeit trägt dazu bei, Schulen/die Kinderund Jugendhilfe zu Orten zu machen, in denen Kinder und Jugendliche selbstbestimmt und frei von Übergriffen sich und ihre Sexualität entwickeln können und dabei von kompetenten Fachkräften begleitet werden.

Datum: 4. März 2015

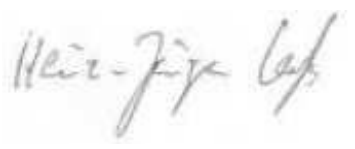

Prof. Dr. Heinz-Jürgen Voß 


\section{Einwilligungserklärung}

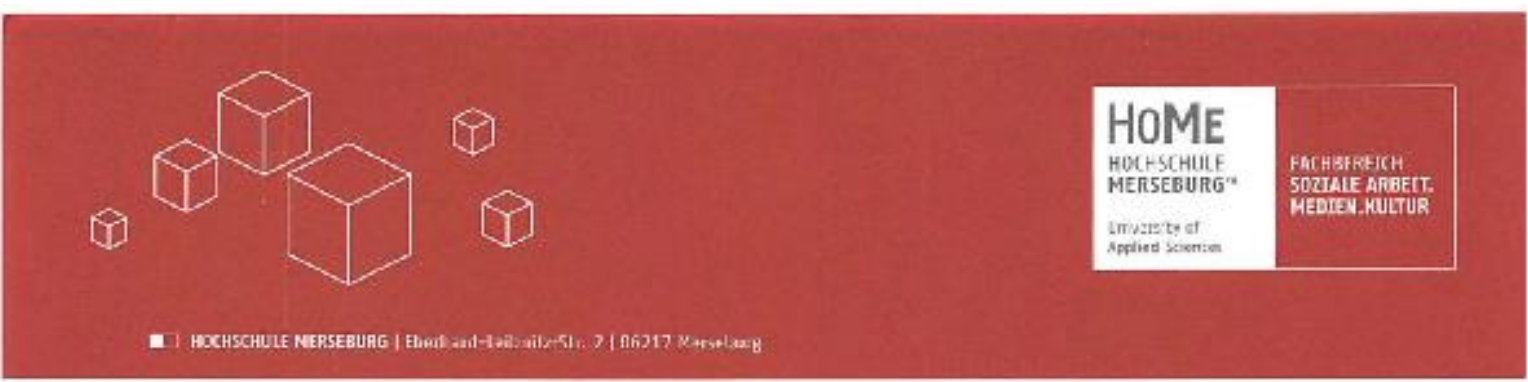

\section{Einwilligungserklärung}

Schutz von Kindern und Jugendlichen vor sexueller Traumatisierung

\section{Forschungsprojekt Schutz von Kindern und Jugendlichen vor sexueller Traumatisierung \\ Institut fur Angewandte \\ Sexualwissenschaft (IfAS)}

Ich bin über das Vorgehen bei der Auswertung der persönlichen "freien" Interviews mit einer Handreichung informiert worden (u.a.: Abbruch des Interviews zu jederzeit möglich; die Abschrift gelangt nicht an die Öffentlichkeit, Anonymisierung bei der Abschrift, Löschung des Tonträgers, Löschung von Namen und Kontaktdaten, Aufbewahrung der Einwilligungserklärung nur im Zusammenhang mit dem Hinweis des Datenschutzes und nicht zusammenführbar mit dem Interview).

Ich bin damit einverstanden, dass meine Aussagen, die aus dem Zusammenhang genommen wurden und damit nicht mehr mit meiner Person in Verbindung gebracht werden können, als Material für wissenschaftliche Zwecke und die Weiterentwicklung der Forschung genutzt werden können. Einzelne Sätze dürfen, unter der Voraussetzung, dass sie nicht mit meiner Person in Zusammenhang gebracht werden können, zitiert werden.

Unter den hier genannten Bedingungen erkläre ich mich bereit, das Interview zu geben und bin damit einverstanden, dass es auf Band aufgenommen, abgetippt, anonymisiert und ausgewertet wird.

Ort, Datum
Fachbereich Soziale Arbeit. Medien. Kultur

\section{ANSCHRIFT}

Hochschule Merseburg (PH)

Geusser Strabe

06217 Merseburg / Deutschland

TELEFON

+493461 46-2208

TELEFAX

$+49346146-2205$

\section{E-MAIL/INTERNET}

heinz-juergen. vossehs-merseburg.de katja.krobik-matthelehs-merseburg.de torsten.linike hhs-merseburg-de wrowi he-marsehurg, de 


\section{Kategoriensystem}

\begin{tabular}{|c|c|c|c|c|}
\hline Nr. & $\begin{array}{c}\text { Zuordnung } \\
\text { deduktiv/induktiv }\end{array}$ & Kategorie & Definition & Ankerbeispiele \\
\hline F1.1 & $\mathrm{d}$ & Sexualaufklärung & $\begin{array}{l}\text { Aussagen zu Elementen } \\
\text { Sexualaufklärung, der } \\
\text { Unterricht vermittelt werden }\end{array}$ & $\begin{array}{l}\text { „Die Fortpflanzungsorgane, anatomisch, } \\
\text { physiologisch, werden behandelt, das ist Thema } \\
\text { männlich, weiblich, alle Prozesse die sich daran } \\
\text { anschließen, jetzt Befruchtungsvorgang, dass man } \\
\text { das erklären kann. Von der Eizelle befruchten, } \\
\text { Eizelle, bis zu Geburt, die ganzen } \\
\text { Entwicklungsstadien."FS1_33 }\end{array}$ \\
\hline F1.2 & $d$ & $\begin{array}{l}\text { Präventionsangebote/- } \\
\text { projekte }\end{array}$ & $\begin{array}{l}\text { Aussagen zu Projekten oder } \\
\text { Angeboten, die mit Fokus auf } \\
\text { Prävention sexualisierter Gewalt } \\
\text { durchgeführt werden }\end{array}$ & $\begin{array}{l}\text { "Und eben auch diese Projekte zum Nein-Sagen, } \\
\text { da gibt es auch verschiedene Anbieter. Das ist also } \\
\text { nicht fest, da probieren wir auch immer ein } \\
\text { Stückchen weit aus, was da funktioniert." FS4_69 }\end{array}$ \\
\hline F1.3 & $d$ & $\begin{array}{l}\text { Schutzkonzept/ } \\
\text { Handlungsleitfaden bei } \\
\text { Verdachtsfällen }\end{array}$ & $\begin{array}{l}\text { Aussagen zu standardisierten } \\
\text { Verfahrensabläufen, die für } \\
\text { Verdachtsfälle vorgesehen sind }\end{array}$ & $\begin{array}{l}\text { "Ich glaube da, also für mich ist es individuell } \\
\text { abhängig. Ähm, ich würde immer mit meiner } \\
\text { Schulleiterin darüber sprechen, wie wir weiter } \\
\text { vorgehen. Da nimmt sie uns glaube ich auch viel } \\
\text { ab. Also jetzt nicht nur was so, so mit Anfassen ist } \\
\text { oder so. Ich würde mit dem Klassenlehrer } \\
\text { sprechen." FS2_91 }\end{array}$ \\
\hline F1.4 & $d$ & Vorgehen im Verdachtsfall & $\begin{array}{lr}\text { Aussagen } & \text { zu } \\
\text { nichtstandardisiertem } & \text { Vorgehen } \\
\text { im Verdachtsfall } & \end{array}$ & $\begin{array}{l}\text { „Ich war, wie sagt man, nur die Fachlehrerin und } \\
\text { sie kam halt zu mir und hat sich mit anvertraut, } \\
\text { dass, ähm, ja zu Hause Probleme, und der } \\
\text { Großvater und belästigt sie halt und sie weiß sich } \\
\text { nicht mehr zu helfen. Zur Oma konnte sie wohl } \\
\text { nicht gehen, weil die Oma ihr das nicht glaubt und } \\
\text { ihre Mutter, es war deren Vater, hatte wohl schon } \\
\text { das gleiche Problem und sie wusste dann gar nicht } \\
\text { mehr weiter und ich habe sie dann, ja /Pro familia } \\
\text { hatte damals keine Zeit, dann sind wir zum } \\
\text { Jugendamt und die haben sich dann weiter drum } \\
\text { gekümmert."FS3_53 }\end{array}$ \\
\hline
\end{tabular}




\begin{tabular}{|c|c|c|c|c|}
\hline F1.5 & d & Verantwortungsgefühl & $\begin{array}{l}\text { Aussagen dazu, inwiefern sich } \\
\text { Lehrkraft in der Verantwortung } \\
\text { fühlt, zur Prävention sexualisierter } \\
\text { Gewalt beizutragen }\end{array}$ & $\begin{array}{l}\text { "Also dass doch mittlerweile sehr viel mehr, ich } \\
\text { sage jetzt mal, in den Nachbarklassenraum } \\
\text { geguckt wird mit mitgeachtet wird, was da passiert } \\
\text { und wenn da gerade mal ein Schüler ausrastet, } \\
\text { dass dann eben gleich ein paar Lehrer auch mit zur } \\
\text { Stelle sind." FS4_23 }\end{array}$ \\
\hline F1.6 & $d$ & $\begin{array}{l}\text { Einbeziehung } \\
\text { Bezugspersonen }\end{array}$ & $\begin{array}{l}\text { Aussagen zur Einbeziehung von } \\
\text { Bezugspersonen (z.B. Eltern) } \\
\text { hinsichtlich der Prävention } \\
\text { sexualisierter Gewalt }\end{array}$ & $\begin{array}{l}\text { "Und zum anderen kann man natürlich versuchen } \\
\text { Eltern einzuladen. Mit den Eltern auch darüber zu } \\
\text { sprechen." FS1_11 }\end{array}$ \\
\hline F1.7 & $d$ & Beziehungsarbeit & $\begin{array}{lr}\text { Aussagen } & \text { über } \\
\text { Beziehungsangebote } & \text { oder } \\
\text { Angebote im Rahmen von } \\
\text { Beziehungsarbeit, die } & \text { als } \\
\text { präventiv erlebt werden } & \\
\end{array}$ & $\begin{array}{l}\text { "Und das braucht dann eine gewisse Zeit, eh die } \\
\text { doch ein bisschen Vertrauen zu dem Klassenlehrer } \\
\text { gewinnen und sich dann eventuell anvertrauen. } \\
\text { Indem sie um einen Gesprächstermin bitten." } \\
\text { FS1_3 }\end{array}$ \\
\hline F1.8 & $d$ & Kooperationspartner*innen & $\begin{array}{l}\text { Aussagen zu bekannten } \\
\text { Kooperationspartner*innen }\end{array}$ & $\begin{array}{l}\text { „In Zusammenarbeit mit der Schulsozialarbeiterin, } \\
\text { das muss man schon sagen. Also die stellt die } \\
\text { Kontakte her und hat da auch ein großes Interesse } \\
\text { dran." FS6_58 }\end{array}$ \\
\hline F2.1 & $d$ & $\begin{array}{l}\text { im/durch } \\
\text { erworbene Kompetenzen }\end{array}$ & $\begin{array}{l}\text { Aussagen über Kompetenzen der } \\
\text { interviewten Person, die diese im } \\
\text { Studium erworben hat und jetzt in } \\
\text { der Vermittlung sexueller Bildung } \\
\text { als hilfreich erlebt werden }\end{array}$ & $\begin{array}{l}\text { "Wir hatten eine Vorlesung zum Thema } \\
\text { Gesundheitserziehung und da wurde das mit } \\
\text { thematisiert." FS3_49 }\end{array}$ \\
\hline F2.2 & $d$ & $\begin{array}{lr}\text { während } & \text { der } \\
\text { Berufstätigkeit } & \text { erworbene } \\
\text { Kompetenzen } & \end{array}$ & $\begin{array}{l}\text { Aussagen über Kompetenzen der } \\
\text { interviewten Person, die diese } \\
\text { während ihrer Berufstätigkeit } \\
\text { erworben hat und jetzt in der } \\
\text { Vermittlung sexueller Bildung als } \\
\text { hilfreich erlebt werden }\end{array}$ & $\begin{array}{l}\text { "Ich war zum Gesprächsschulung nochmal, das } \\
\text { waren } 3 \text { Tage, aber da war das eben sehr } \\
\text { praktisch, da hatten wir nochmal hier diese ganzen } \\
\text { Bausteine und die mussten wir auch nochmal } \\
\text { ausprobieren da ist das nicht in 120min erledigt." } \\
\text { FS5_182 }\end{array}$ \\
\hline F2.3 & $d$ & persönliche Eigenschaften & $\begin{array}{l}\text { Aussagen über persönliche } \\
\text { Eigenschaften der interviewten } \\
\text { Person, die als Ressource } \\
\text { wahrgenommen werden }\end{array}$ & $\begin{array}{l}\text { "Ich glaube, dass ich ein, ein irgendwo sehr } \\
\text { feinfühliger Mensch bin. So ein gewisses Gespür } \\
\text { habe, wenn es einem Kind nicht gut geht." } \\
\text { FS2_100 }\end{array}$ \\
\hline
\end{tabular}




\begin{tabular}{|c|c|c|c|c|}
\hline F2.4 & d & fehlende Kompetenzen & $\begin{array}{l}\text { Aussagen über Wissenslücken } \\
\text { oder anderweitig fehlende } \\
\text { Kompetenzen in der Vermittlung } \\
\text { von Sexueller Bildung, die als } \\
\text { notwendig erachtet werden, aber } \\
\text { nicht vorhanden sind }\end{array}$ & $\begin{array}{l}\text { „Ja, (..) ja, wie gehe ich mit dem Kind um, äh, das } \\
\text { ist die Frage. Was (...) / Soll ich mir alles erzählen } \\
\text { lassen, soll ich die Eltern mit einbeziehen?“ } \\
\text { FS3_91 }\end{array}$ \\
\hline F2.5 & $d$ & $\begin{array}{l}\text { Kompetenzen im } \\
\text { Kollegium }\end{array}$ & $\begin{array}{l}\text { Aussagen über besondere } \\
\text { Kompetenzen im Kollegium, die } \\
\text { als Ressource in der Vermittlung } \\
\text { Sexueller } \\
\text { wahrgenommen werden }\end{array}$ & $\begin{array}{l}\text { "Also ich bin und ich denke, da kann ich auch für } \\
\text { die meisten anderen sprechen, es gibt keinen } \\
\text { Punkt in unserer Ausbildung als Lehrer, wo das } \\
\text { eine Rolle spielt." FS4_124 }\end{array}$ \\
\hline F2.6 & $d$ & Verweisungskompetenzen & $\begin{array}{l}\text { Aussagen über eigene } \\
\text { Verweisungskompetenzen zu } \\
\text { spezialisierten Personen, z.B. } \\
\text { Fachberatungsstelle }\end{array}$ & $\begin{array}{l}\text { "Ja, ich verweise auf Beratungsstellen (...).“ } \\
\text { FS1_13 }\end{array}$ \\
\hline F2.7 & $d$ & Ängste & $\begin{array}{l}\text { Aussagen über Ängste der } \\
\text { interviewten Person im Hinblick } \\
\text { auf Vermittlung Sexueller Bildung }\end{array}$ & $\begin{array}{l}\text { „Wenn ich von jemandem was erfahre, wo wende } \\
\text { ich mich denn hin? Ähm, wo mache ich mich denn } \\
\text { strafbar, wenn ich das Kind dann näheres gefragt } \\
\text { habe oder so? So'ne Sachen, die kennen wir ja } \\
\text { überhaupt nicht." FS2_27 }\end{array}$ \\
\hline F2.8 & $d$ & Vulnerabilität & $\begin{array}{l}\text { Aussagen zu persönlicher } \\
\text { Vulnerabilität im Kontext von } \\
\text { sexualitätsbezogenen Themen }\end{array}$ & $\begin{array}{l}\text { „Man darf nicht alles mit nach Hause nehmen. Also } \\
\text { man muss auch eine bestimmte, es geht ihnen } \\
\text { sicher auch so, was sie sicher auch so, was sie } \\
\text { sicher alles so hören, sehen, man kann das nicht } \\
\text { alles mit nach Hause nehmen. Dann würde man } \\
\text { kaputtgehen, ja. Aber es gibt schon Fälle, wo mich } \\
\text { das sehr erschüttert, was ich so." FS2_104 }\end{array}$ \\
\hline F3.1 & $d$ & $\begin{array}{l}\text { Sexualität im Lebensraum } \\
\text { Schule }\end{array}$ & $\begin{array}{l}\text { Aussagen über Umgang mit } \\
\text { Sexualität von Schülerinnen im } \\
\text { Schulalltag }\end{array}$ & $\begin{array}{l}\text { "Also ich denke mir so, so als 13-Jährige haben } \\
\text { Sex miteinander und brüsten sich damit, erzählen } \\
\text { das auch." FS6_41 }\end{array}$ \\
\hline F3.2 & $d$ & Toleranz & $\begin{array}{l}\text { Aussagen zur Verhandlung von } \\
\text { Themen zu sexueller Vielfalt im } \\
\text { Schulalltag }\end{array}$ & $\begin{array}{l}\text { "Dann geht es schon noch konkret darum, } \\
\text { Orientierung, sexuelle Orientierung, also dass das } \\
\text { auch verschiedene Möglichkeiten gibt." FS4_73 }\end{array}$ \\
\hline F3.3 & $d$ & Akzeptanz & $\begin{array}{lll}\text { Aussagen } & & \text { zur } \\
\text { Auseinandersetzung } & \text { mit } & \text { der }\end{array}$ & $\begin{array}{l}\text { „Das ist halt in unserer Arbeit vor allen Dingen mit } \\
\text { unseren Mädchen ein ganz wichtiges Thema, das }\end{array}$ \\
\hline
\end{tabular}




\begin{tabular}{|c|c|c|c|c|}
\hline & & & $\begin{array}{ll}\text { Akzeptanz } & \text { alternativer } \\
\text { Lebensmodelle } & \end{array}$ & $\begin{array}{l}\text { sie sehr, sehr oft, sehr, naja, sehr bereitwillig } \\
\text { einfach, und gerade je älter sie werden eben auch } \\
\text { mit sehr vielen wechselnden Partnern mit ihrem } \\
\text { Körper einfach umgehen."FS4_3 }\end{array}$ \\
\hline F3.4 & $d$ & Grenzverletzungen & $\begin{array}{l}\text { Aussagen zu sexuellen } \\
\text { Grenzverletzungen innerhalb der } \\
\text { Schule }\end{array}$ & $\begin{array}{l}\text { "Ja, das gibt eine sehr sexualisierte Sprache. Auch } \\
\text { uns gegenüber. Also ich bin schon öfter mal die } \\
\text { alte Fotze und das Tittenmonster und so." FS6_41 }\end{array}$ \\
\hline F3.5 & $d$ & Partizipation & $\begin{array}{l}\text { Aussagen zur Partizipation von } \\
\text { Schüler*innen hinsichtlich ihrer } \\
\text { sexuellen Selbstbestimmung }\end{array}$ & $\begin{array}{l}\text { „Naja, es gibt ja einen Klassensprecher, der könnte } \\
\text { jetzt sich an die pädagogische Leitung wenden und } \\
\text { sagen, ja, das und das Problem bestände in der } \\
\text { Klasse und um eine Lösung zu bitten, ja." FS1 } 77\end{array}$ \\
\hline F3.6 & $d$ & Grenzen & $\begin{array}{l}\text { Aussagen zu Grenzsetzungen } \\
\text { hinsichtlich des Themenbereichs } \\
\text { Sexualität/sexuelle } \\
\text { Selbstbestimmung }\end{array}$ & $\begin{array}{l}\text { Das sind so Sachen wo ich mir sage, das kann } \\
\text { nicht sein. Also sind schon Dinge, wo, wo man, die } \\
\text { man mit nach Hause nimmt und wo man darüber } \\
\text { nachdenkt und auch nicht schlafen kann oder so. }\end{array}$ \\
\hline F3.7 & $d$ & Haltung & $\begin{array}{l}\text { Aussagen, die Haltung der } \\
\text { interviewten Person zu Sexualität } \\
\text { von Schüler*innen aufzeigt }\end{array}$ & $\begin{array}{l}\text { "Weil die sich auch immer mehr untereinander } \\
\text { wiederfinden zu neuen Beziehungen, ich will gar } \\
\text { nicht sagen Familien, weil es diese Struktur nicht } \\
\text { hält, zu neuen Beziehungen und neuen } \\
\text { Elternschaften gründen und so ne. Vermischt sich } \\
\text { nicht mehr, Es ist, es wird immer schwieriger.“ } \\
\text { FS6_29 }\end{array}$ \\
\hline F4.1 & $d$ & $\begin{array}{l}\text { Erfahrungen mit Fort- } \\
\text { und Weiterbildung }\end{array}$ & $\begin{array}{l}\text { Aussagen über Erfahrungen } \\
\text { hinsichtlich Teilnahme an, Inhalt } \\
\text { von, ... Fort- und Weiterbildungen }\end{array}$ & $\begin{array}{l}\text { „Also wir hatten auch mal (...) eine Fortbildung zu } \\
\text { dem Thema im weitesten Sinne. Also es geht da } \\
\text { (...) mehr um dieses Erkennen von wirklichem } \\
\text { Missbrauch, sexuellen Missbrauchsfällen. Und } \\
\text { was wir da sozusagen tun können, was da unsere } \\
\text { Möglichkeiten, Rechte, Pflichten sind." FS4_128 }\end{array}$ \\
\hline F4.2 & $d$ & Praktikabilität & $\begin{array}{l}\text { Aussagen zur Umsetzbarkeit der } \\
\text { Inhalte im Schulalltag }\end{array}$ & $\begin{array}{l}\text { „Man kann das nach dem Unterricht machen, da } \\
\text { kann man maximal noch zwei Stunden etwas } \\
\text { erzählen, weil dann sind die Lehrer, wenn die } \\
\text { sechs Stunden Unterricht gemacht haben auch } \\
\text { einfach fertig. Und man kann das halt in einem }\end{array}$ \\
\hline
\end{tabular}




\begin{tabular}{|c|c|c|c|c|}
\hline & & & & $\begin{array}{l}\text { größeren Rahmen machen, also sprich in einer } \\
\text { Tagesveranstaltung, ist aber unrealistisch, weil das } \\
\text { muss man dann in der Regel irgendwo in die Ferien } \\
\text { legen." FS4_142 }\end{array}$ \\
\hline F4.3 & $d$ & Bedarfe & $\begin{array}{l}\text { Aussagen zu Bedarfen an Fort- } \\
\text { und Weiterbildungen }\end{array}$ & $\begin{array}{l}\text { „Ja, wie geht man mit diesem Wissen, wenn sie } \\
\text { sich anvertrauen, um? Was kann man machen? } \\
\text { Wo kann man sie hinschicken? In welche Richtung } \\
\text { könnte das gehen?" FS1 } 51\end{array}$ \\
\hline F4.4 & $d$ & Schwierigkeiten & $\begin{array}{l}\text { Aussagen zu Schwierigkeiten, die } \\
\text { im Kontext von Fort- und } \\
\text { Weiterbildungen erlebt werden }\end{array}$ & $\begin{array}{l}\text { "Wenn du vormittags unterrichtet hast, dann ist die } \\
\text { Aufnahmefähigkeit am Nachmittag nicht mehr so } \\
\text { günstig." FS5 } 177\end{array}$ \\
\hline
\end{tabular}




\section{Eidesstattliche Erklärung}

Ich erkläre hiermit, dass ich die vorliegende Arbeit selbstständig angefertigt habe. Die aus fremden Quellen direkt oder indirekt übernommenen Gedanken sind als solche einzeln kenntlich gemacht. Es wurden keine anderen als die von mir angegebenen Quellen und Hilfsmittel (inklusive elektronischer Medien und Online-Ressourcen) benutzt.

Die Arbeit wurde bisher keiner anderen Prüfungsbehörde vorgelegt und auch nicht veröffentlicht.

Ich bin mir bewusst, dass eine falsche Erklärung zur Note „ungenügend“ führt und rechtliche Folgen nach sich ziehen kann.

Merseburg, den Unterschrift 